\title{
Application of omics to immunotoxicology : from mechanisms of action to alternative testing strategies
}

Citation for published version (APA):

Shao, J. (2015). Application of omics to immunotoxicology : from mechanisms of action to alternative testing strategies. [Doctoral Thesis, Maastricht University]. Uitgeverij BOXPress.

https://doi.org/10.26481/dis.20150211js

Document status and date:

Published: 01/01/2015

DOI:

10.26481/dis.20150211js

Document Version:

Publisher's PDF, also known as Version of record

\section{Please check the document version of this publication:}

- A submitted manuscript is the version of the article upon submission and before peer-review. There can be important differences between the submitted version and the official published version of record.

People interested in the research are advised to contact the author for the final version of the publication, or visit the DOI to the publisher's website.

- The final author version and the galley proof are versions of the publication after peer review.

- The final published version features the final layout of the paper including the volume, issue and page numbers.

Link to publication

\footnotetext{
General rights rights.

- You may freely distribute the URL identifying the publication in the public portal. please follow below link for the End User Agreement:

www.umlib.nl/taverne-license

Take down policy

If you believe that this document breaches copyright please contact us at:

repository@maastrichtuniversity.nl

providing details and we will investigate your claim.
}

Copyright and moral rights for the publications made accessible in the public portal are retained by the authors and/or other copyright owners and it is a condition of accessing publications that users recognise and abide by the legal requirements associated with these

- Users may download and print one copy of any publication from the public portal for the purpose of private study or research.

- You may not further distribute the material or use it for any profit-making activity or commercial gain

If the publication is distributed under the terms of Article $25 \mathrm{fa}$ of the Dutch Copyright Act, indicated by the "Taverne" license above, 


\title{
Application of omics to immunotoxicology: from mechanisms of action to alternative testing strategies
}

\author{
Jia Shao
}


Jia Shao

Application of omics to immunotoxicology: from mechanisms of action to alternative testing strategies Thesis Maastricht University, Maastricht

ISBN 978-94-6295-078-8 


\title{
Application of omics to immunotoxicology: from mechanisms of action to alternative testing strategies
}

\author{
PROEFSCHRIFT \\ ter verkrijging van de graad van doctor aan de Universiteit Maastricht, \\ op gezag van de Rector Magnificus, Prof. dr. L.L.G. Soete \\ volgens het besluit van het College van Decanen, \\ in het openbaar te verdedigen \\ op woensdag 11 februari 2015 om 10:00 uur \\ door \\ Jia Shao \\ "Geboren te Beijing op 1 november 1984"
}




\section{Promotor}

Prof. dr. Henk van Loveren

\section{Co-promotor}

Dr. Ad Peijnenburg, RIKILT Wageningen UR

\section{Beoordelingscommissie}

Prof. dr. Edwin Mariman (voorzitter)

Prof. dr. Bas Blaauboer, Universiteit Utrecht

Dr. Janine Ezendam, RIVM

Prof. dr. Jos Kleinjans

Dr. Theo de Kok

\section{Netherlands 2 ,}

Toxicogenomics I CENTRE 


\section{Table of Contents}

Chapter 1 General introduction

Section I Potential mechanisms of action underlying chemical-induced direct immunotoxicity

Chapter 2 Toxicogenomics-based identification of mechanisms for direct immunotoxicity

Chapter 3 Protein phosphorylation profiling identifies potential mechanisms for direct immunotoxicity

Chapter 4 Inhibition of CXCL12-mediated chemotaxis of Jurkat cells by direct immunotoxicants

Chapter 5 Microarray analysis of human peripheral blood mononuclear cells (PBMCs) treated with tributyltin-oxide (TBTO)

Section II Identification and validation of functional biomarkers for predictive immunotoxicity testing

Chapter 6 Transcriptome-based functional classifiers for direct immunotoxicity

Chapter 7 Successful validation of genomic biomarkers for human immunotoxicity in Jurkat T cells in vitro

Chapter 8 Summary, General Discussion, Future Perspectives, and Conclusions

Chapter 9 Samenvatting 



\section{CHAPTER 1}

General Introduction 


\section{The immune system}

The immune system detects a wide range of agents, from infectious microorganisms to other foreign substances, and protects the body against diseases. This is done on the basis of recognition of self and response to non-self (Schultz and Grieder, 1987). Proper functioning of the immune system requires the crosstalk among lymphoid organs, immune cells, humoral factors, and cytokines. The organs of the immune system, or the so called lymphoid organs, are positioned throughout the body. Based on the stage of lymphocyte development and maturation lymphoid organs are involved in, they can be divided into primary and secondary, also called central and peripheral, lymphoid organs. Primary lymphoid organs are bone marrow and thymus, which generate lymphocytes from immature progenitor cells. Lymphoid organs such as lymph nodes, Peyer's patches, and spleen, belong to secondary lymphoid organs, which maintain mature naive lymphocytes and provide the environment for antigens to interact with lymphocytes, in order to initiate adaptive immune responses (Turley et al., 2010).

Depending on the speed and specificity of the response, two major strategies can be found in immunity, being innate and adaptive immunity, also known as non-specific and specific (or acquired) immunity. Although the functions of these two types of immunity are distinctive from each other, there is much interaction between them, i.e. components of the innate immune system can influence the adaptive immune system and vice versa.

\subsection{Innate immunity}

The innate immune system is composed of innate leukocytes including natural killer cells, mast cells, eosinophils, basophils, and phagocytic cells including neutrophils, macrophages, and dendritic cells; and other elements of the immune system, including complement system, cytokines, and acute phase proteins (Janeway et al., 2001). Compared to the adaptive immune system, the innate immune system is not specific to a particular pathogen, and therefore, is constitutively present and readily initiated. It serves as the first line of defence of the body against invading organisms or foreign substances (Janeway et al., 2001).

During the very early stage of infection or tissue damage, activated macrophages release cytokines, which stimulate the division of myeloid precursors in the bone marrow, causing neutrophil leukocytosis. Neutrophils use a multistep process dependent on interaction between proinflammatory mediators, adhesion molecules, chemoattractants, and chemokines to home to a site of infection. They are typically the first leukocytes to be recruited to the infection site, and they phagocytose organsisms by making pseudopodia (projections of cytoplasmic membrane). The recruitment and activation of neutrophils at the site of infection is a central feature of the innate immune response. Several lines of evidence also indicated that the inappropriate occurring of this process leads to chronic inflammation (Kolaczkowska and Kubes, 2013). 


\subsection{Adaptive immunity}

The major characteristics of adaptive immunity are the use of antigen-specific receptors on $\mathrm{T}$ and $\mathrm{B}$ lymphocytes to initiate targeted immune responses and the creation of immunological memory to a specific pathogen, which leads to an enhanced response when encountering the same pathogen. Depending on the cells involved, adaptive immunity can be further divided into humoral immunity which involves B lymphocytes, and cell-mediated immunity that involves $T$ lymphocytes. B and $T$ lymphocytes are derived from the same multipotent hematopoietic stem cells, and are the major cell types involved in adaptive immunity. B cells remain in the bone marrow during their development, whereas $T$ progenitor cells migrate to the thymus as thymocytes and develop into $T$ cells. Mature $B$ and $T$ lymphocytes are transported by blood and lymph to other lymphoid organs, such as the lymph nodes and the spleen.

Both B and T lymphocytes can be activated when they encounter and bind to an antigen. Antigens are brought to the lymphoid tissue directly in the lymph or by antigen presenting cells (APCs), including dendritic cells, macrophages, and B lymphocytes that have endocytosed the antigen. One crucial difference between $B$ and $T$ lymphocytes is the way that they recognize their antigen. $B$ lymphocytes recognize free antigens in the blood or lymph using B cell receptors or via membrane bound immunoglobulins. In contrast, $\mathrm{T}$ lymphocytes recognize antigens through $\mathrm{T}$ cell receptors after they have been processed by APCs and presented as peptide fragments via human leukocyte antigen (HLA) molecules at the surface of APCs (Parkin and Cohen, 2001).

The major functions of B lymphocytes include antibody production, antigen presentation, and development into memory B cells (Janeway et al., 2001). Recently, it has also been discovered that B lymphocytes are capable of releasing a broad range of cytokines that regulate immune responses (Mauri and Bosma, 2012).

The recognition of antigen is different for $\mathrm{CD} 4^{+}$(helper) and $\mathrm{CD}^{+}$(cytotoxic) $\mathrm{T}$ lymphocytes, which are two major types of effector T lymphocytes. CD4 lymphocytes only recognize antigens presented with HLA class II (exogenous antigens) and CD8 lymphocytes only with HLA class I (endogenous antigens, such as viral or tumour proteins). In addition, co-receptor stimulation is needed for the activation of $\mathrm{T}$ lymphocytes. These mechanisms ensure the precise recognition of foreign antigens. Activation of $\mathrm{CD}^{+} \mathrm{T}$ lymphocytes initiate cytotoxic attack in order to remove the cells that are infected by virus or other intracellular pathogens, or are producing tumour antigens. Whereas activation of $\mathrm{CD}^{+} \mathrm{T}$ lymphocytes leads to production of cytokines that in turn activate other leukocytes including $\mathrm{B}$ lymphocytes, $\mathrm{CD}^{+} \mathrm{T}$ lymphocytes, and macrophages (Parkin and Cohen, 2001). Depending on the pattern of cytokines they produce, $\mathrm{CD}^{+} \mathrm{T}$ lymphocytes can be further divided into Th1 cells that produce for instance interleukin 2 (IL2) and interferon Y (IFNY) which induce mainly cell-mediated immune responses, and Th2 cells that produce interleukin 4, 5, 6, and 10 (IL4, IL5, IL6, and IL10) that enhance antibody production, and are involved in allergic conditions (Swain et al., 1991). Other types of $\mathrm{T}$ lymphocytes include memory $\mathrm{T}$ lymphocytes, which are antigen-specific $\mathrm{T}$ lymphocytes that 
survived from infection, and regulatory $\mathrm{T}$ lymphocytes, which express FoxP3 molecule and are involved in regulation of T cell-mediated immune responses (Vignali et al., 2008).

\section{Immunotoxicity}

Immunotoxicity refers to any adverse effects on the structure or function of the immune system that result from exposure to xenobiotics including pharmaceuticals (De Jong and Van Loveren, 2007; Lankveld et al., 2010). Immunotoxicity can be induced either directly or indirectly. Direct immunotoxicity is caused by the effects of chemicals on components of the immune system, resulting in immunosuppression or immunostimulation, whereas indirect immunotoxicity refers to specific immune response to a compound itself or to a self-antigen altered by the compound, leading to hypersensitivity or autoimmunity (Lankveld et al., 2010). The differentiation between direct and indirect immunotoxicity is often not clear, as some compounds, for instance the heavy metal mercury, not only have a direct effect on the immune system but also alter the immune responses (Schwenk et al., 2009). The present thesis mainly focused on chemical-induced direct immunotoxicity.

\subsection{Chemical-induced immunosuppression vs. immunostimulation}

Immunosuppression is the consequence of a reduced response of the immune system, whereas immunostimulation results from increased activity of any components or activation of the immune system. Both of them can be either unexpected or desirable. Unexpected immunosuppression may reduce the body's resistance against infectious diseases and development of malignancies; and unexpected immunostimulation leads to more frequent autoimmune diseases and hypersensitivity reactions to unrelated allergens (Descotes, 2005). On the other hand, immunosuppression induced by administration of immunosuppressive agents may enhance the therapeutic benefits of treatments for allergies and autoimmune diseases and prevent rejection of transplanted organs; and immunostimulatory agents also have their therapeutic application in the treatment of infections and cancer.

\subsection{Human exposure}

Compounds with direct immunotoxic properties include metals, mycotoxins, agricultural pesticides, and industrial chemicals. They form potential risk to human health due to exposure through food, drinking water, and the environment.

Short term high level of exposure (acute poisoning) to the above mentioned toxins can result in a broad range of symptoms including death in adult and children, depending on the type of toxin and exposure route. Regarding chronic toxicity on the immune system, occupational exposure to 
polycyclic aromatic hydrocarbons (PAHs) has been found to modulate $\mathrm{T}$ lymphocyte proliferation and NK cell activity (Karakaya et al., 2004). Occupational exposure to mercury compounds have been associated with increased circulating levels of autoantibodies (Alves et al., 2006; Gardner et al., 2010; Silva et al., 2004) and increased risks of autoimmune diseases (Cooper et al., 2004). Immune alterations have also been reported in the populations exposed to polychlorinated biphenyls (PCBs), polychlorinated dibenzofurans (PCDFs), and polychlorinated quaterphenyls (PCQs) via ingestion of contaminated rice oil in Japan (Yusho) and Taiwan (Yu Cheng) (Chang et al., 1981; Chang et al., 1982; Kuratsune et al., 1972; Lü and Wu, 1985). In about half of the Yu Cheng cases, persistent respiratory distress was observed, accompanied by Gram-negative bacilli infection. Significant decreases in serum IgA and IgM levels and the percentage of total T lymphocytes were observed in the follow up check-ups after 1, 2 and 3 years. Even after 14 years of exposure, the Yusho patients still showed differences in their $\mathrm{T}$ cell lineage proportions as compared to the control.

Another important aspect regarding the toxicity of certain toxins is the transplacental transport, which may affect the development of the immune system in uteor. Heavy metals like $\mathrm{MeHg}$ can easily pass the placenta and accumulate in the fetus because the fetus does not have the ability to excrete mercury. A population surveillance study on $\mathrm{MeHg}$ exposures in the Brazilian Amazon provided evidence by measuring immunoglobulin and cytokine levels that chronic prenatal exposure to low levels of mercury compounds induced immunotoxicity in both pregnant women and their fetuses (Nyland et al., 2011). PCBs are also known to cross placenta and to be excreted into the maternal milk. Epidemiological studies of Great Lakes fish-eating cohorts have indicated that breast-fed infants whose mothers consumed high amount of fish were more prone to microbial infections than breastfed infants whose mothers did not eat fish (Humphrey, 1988). More recent studies of BraMat, a subcohort of the Norwegian Mother and Child Cohort study (MoBa) also showed that prenatal dietary exposures to PCBs and dioxins may increase the risk of wheeze and infectious diseases in the offspring during the first year of life (Stølevik et al., 2011), and reduce antibody response to measles vaccine at the age of three (Stølevik et al., 2013).

\section{Current testing strategies for immunotoxicity}

The assessment of direct immunotoxicity is an important component of the overall toxicological investigation during the safety assessment of chemicals and food additives (EPA, 1998; Institóris et al., 1998) and the preclinical phase of drug development (FDA, 2002; ICH, 2006). Numerous experimental approaches are available for detecting and studying the direct immunotoxic effects of chemicals, most of which are based on in vivo toxicity tests with rodent-based animal models.

\subsection{In vivo tests for immunotoxicity}


A tiered approach has been recommended for in vivo immunotoxicological tests. TIER I being a 28day repeated dose toxicity study with emphasis on evaluation of immune-related organs. The Test Guideline No. 407 from OECD (Organisation for Economic Cooperation and Development) indicated the possibility of obtaining information regarding immunotoxicity through the analysis of several parameters accepted in standard toxicity studies, such as: weight and histopathological evaluation of lymphoid organs (thymus, spleen, draining lymph node and at least one additional lymph node), histological evaluation of bone marrow, peripheral white blood cell count and differentiation, and immunoglobulin serum levels (OECD, 2008). In addition, FDA proposed that examination of the lymphoid tissues that drain or contact the site of drug administration should be performed as well. These include the gut-associated lymphoid tissues (GALT) for orally administered drugs, bronchusassociated lymphoid tissues (BALT) for inhaled drugs, nasal-associated lymphoid tissue (NALT) for inhaled drugs or drugs administered via nasal route, and the regional draining lymph node for drugs administered dermal, intradermal, intramuscularly, or subcutaneously (FDA, 2002). If a chemical shows immunotoxicity during the TIER I studies, additional experiments in order to determine the effects of the test chemical on more detailed immune function should be considered in TIER II studies.

During TIER II studies, immune responses to various types of antigens will be determined, which include T cell-dependent and -independent antigens (De Jong and Van Loveren, 2007). Two wellestablished assays that belong to the former category is the anti-sheep red blood cell plague-forming cell (PFC) assay, and the newer model, anti-keyhole limpet hemocyanin (KLH) antibody ELISA (Ladics, 2007; Tetko et al., 2008). The PFC assay has been extensively evaluated by the National Toxicology Program (NTP), so that an extremely large database of published results is available (Luster et al., 1988; Luster et al., 1993; Luster et al., 1992). However, this assay does not use a wellstandardized antigen, is time-consuming, and can only be applied in rodents. In contrast, the KLH antibody ELISA uses a well-standardized antigen and can be applied in all mammal species including non-rodents (Gevaert et al., 2008). Therefore, it is nowadays more commonly used as the preferred T cell-dependent antibody response (TDAR) assay. Some modifications of the plague forming assay are also available, which can also be used to determine immune response to $T$ cell-independent antigens, such as lipopolysaccharide (LPS) (Holsapple, 1995).

Immunophenotyping of lymphoid tissue, spleen and thymus in particular, and/or peripheral blood, can yield additional information regarding the cell population targeted by the test compound. This type of test can be achieved by flow cytometry or immunohistochemical analysis, and can be easily incorporated in TIER I tests (FDA, 2002; ICH, 2006; Oughton and Kerkvliet, 2005).

Host resistance studies examine the effects of test chemical on the immune system when it is challenged by bacterial, viral, or parasitic infection at the same time. It is expected that direct immunotoxicants will induce immunosuppression and thus cause increase in rate and/or severity of the infection. These tests have the advantages that the innate immunity is also involved. Furthermore, 
the results from these tests can be used to identify and/or confirm the cell population affected by the test chemical (De Jong and Van Loveren, 2007; ICH, 2006).

In addition to the above mentioned assays, the functions of NK cells, macrophages, and neutrophils can also be tested in several ex vivo assays, in which the cells were taken from the animals treated with test chemical $(\mathrm{ICH}, 2006)$.

The animal-based approach, however, has several disadvantages, including generally low sensitivity and limited prediction towards the human situation due to interspecies variability in the immune system. Several tests that were involved in TIER I studies have been demonstrated as relatively poor and unreliable predictors, such as thymus weight, spleen cellularity, and white blood cell count (Luster et al., 1992). Furthermore, the changing of the immune system during aging, for instance, the decrease in thymus weight; and during different functional states, for instance, antibody production after infection, makes the evaluation very difficult (De Jong and Van Loveren, 2007). The use of animals also requires high cost in terms of both time and money and raises ethical concerns in society (Dobrovolskaia and McNeil, 2013). Therefore, the development of in vitro testing methods as alternatives for animal models of human toxicity is being promoted by EU policy (Bouvier d'Yvoire et al., 2012).

\subsection{In vitro immunotoxicity tests}

The report of a workshop, which was held by the European Center for the Validation of Alternative Methods (ECVAM) in 2003, reviewed the state-of-the-art of in vitro testing in the field of immunotoxicity (Gennari et al., 2005). In line with the in vivo approach, the ECVAM workshop has recommended a flow chart/ decision tree approach for in vitro immunotoxicity testing. This approach was made on the basis of the distinction between myelotoxicity and lymphotoxicity (Lankveld et al., 2010).

Bone marrow contains hematopoietic stem cells, which give rise to all immune cells. If a test compound is capable of damaging or destroying the bone marrow, it can be expected that this compound will express immunotoxic effects as well because of the deficiency of immune cells. Thus, it is logical to start the immunotoxicity screening with the evaluation of myelotoxicity. And if a compound is classified as a myelotoxicant, there will be no need to proceed with additional immunotoxicity tests, as this compound will be classified as an immunotoxicant (Gennari et al., 2005). For evaluating the myelotoxic properties of compounds, the in vitro colony forming units-granulocyte/ macrophage (CFU-GM) assay, which has been validated by ECVAM, can be applied. In this assay, the number of surviving bone marrow progenitors will be quantified and used as a functional endpoint for myelotoxicity evaluation (Pessina et al., 2003; Pessina et al., 2001).

A non-myelotoxic compound may still be toxic to the lymphocytes and thus contribute to immuntoxicity. Thus, the second step in in vitro evaluation of direct immunotoxicity should be focused 
on lymphotoxicity. Lymphotoxicity can be caused by inhibition of differentiation and activation of lymphocytes, but can also be caused by inducing cell death. Therefore, the viability of lymphocytes in response to a test compound should be tested at the beginning, in order to determine sub-cytotoxic concentration of the test compound, which can be used in lymphocyte functional tests. The most reliable functional tests for lymphotoxicity have been proven to be the whole blood cytokine release assay and the lymphocyte proliferation test (Lankveld et al., 2010). The former has been pre-validated for application in in vitro immunotoxicity testing (Langezaal et al., 2002). The ECVAM workshop has recommended to increase the value of this assay by extending the current measurement of two cytokines, being IL-1 $\beta$ and IL-4, secreted by monocytes and Th2-lymphocytes, respectively, to a broad panel of cytokines (Gennari et al., 2005).

An important characteristic of lymphocytes is that they proliferate during adaptive immune responses. Therefore, suppression in lymphocyte proliferation may be indicative for immunotoxicity. T lymphocytes proliferation assay measures the effects of test compound on the proliferation of $T$ cells in response to mitogens (ConA or PHA) or antigens (combination of anti-CD3 and anti-CD28). For B cells, mitogens LPS or STM, or the combination of anti-CD40 and IL-4 can be used for proliferation stimulation (International Programme on Chemical Safety (IPCS), 1996). Alternatively, proliferation of T lymphocytes can be assessed in mixed lymphocyte reaction (MLR), in which T cells are stimulated to proliferate by being co-cultured with allogeneic stimulator cells (Elves and Israëls, 1965).

Other approaches for lymphotoxicity evaluation include:

- Cytotoxic T-lymphocyte assay (CTL). CTL assay is a continuation of MLR, in which T lymphocytes are further differentiated into $\mathrm{CD}^{+}$lymphocytes with specific cytotoxicity for target cells possessing intracellular antigens, for instance tumor cells and infected cells. Both splenocytes and peripheral blood lymphocytes can be used for this assay. CTL assay is one of the few assays that capable of evaluating effective antigen presentation via both MHC class I and II molecules (Dietert et al., 2010a).

- $T$ cell-dependent antibody response. Production of a $T$ cell-dependent antibody response is always considered as the "golden standard" in animals. The application of Mishell-Dutton culture, which used mice splenocytes, has been reported to correctly classify six (out of seven) immunosuppressive compounds and four non-immunotoxic compounds (Koeper and Vohr, 2009). Further exploration of this model system is still needed. However, there are currently no good model systems for in vitro antibody production using human cells.

- Dendritic cell (DC) maturation. DCs contribute to the initiation of adaptive immune responses by promoting activation of naive $\mathrm{T}$ cells. $\mathrm{DC}$ can be found in two stages, the immature stage for antigen uptake and mature stage for antigen presentation. The maturation of DC is determined by assessing the expression levels of surface markers, such as CD54 and CD86. It has been demonstrated recently that the environmental immunotoxicant lead, as well as several anti-inflammatory drugs, can alter the immunophenotype and cytokine expression of 
DCs (Dietert et al., 2010b; Toebak et al., 2008), which provided evidence that DCs can also be a target of immunotoxicants.

- Natural killer cell assay. Since NK cells play a crucial role in innate immunity, assessing the effects of test compound on the activity of NK cells is commonly recommended as part of the in vitro immunotoxicity evaluation. NK cells are harvested from peripheral blood of treated animals; and their cytotoxic function is examined using labelled target cells or four-color flow cytometry-based cytotoxicity assay (FCC) (Kim et al., 2007). The activity of NK cells are highly sensitive in response to toxicant exposure, therefore, this assay is usually included in toxicological assessment $(\mathrm{ICH}, 2006)$. However, changes in NK cell activity are nonspecific reactions and sometimes associated with various conditions of unknown etiology rather than pathological conditions of the immune system (Stewart et al., 2003).

- Fluorescent cell chip. For measuring cytokine expression profiles, a new in vitro system, "fluorescent cell chip", has been developed. This system is consisted of cell lines derived from T cells, mast cells, and macrophage-monocytes, which are transfected with a number of cytokine reporter cell constructs (Ringerike et al., 2005). Although further refinement in terms of expanding cell types and cytokines is still needed, this approach is promising for in vitro screening of chemicals for their immunotoxic properties.

TABLE 1. Key targets of different in vitro assays for chemical-induced immunotoxicity.

\begin{tabular}{|c|c|c|c|}
\hline Key Target & In vitro assays & Validation & References \\
\hline Bone marrow & CFU-GM & validated & $\begin{array}{l}\text { (Haglund et al., 2010; Rich and Hall, } \\
\text { 2005) }\end{array}$ \\
\hline Innate immunity & $\begin{array}{c}\text { whole blood cytokine release } \\
\text { Fluorescent cell chip } \\
\text { Natural killer cell activity }\end{array}$ & $\begin{array}{c}\text { pre- } \\
\text { validated } \\
\text { not yet }\end{array}$ & $\begin{array}{l}\text { (Langezaal et al., 2002) } \\
\text { (Ringerike et al., 2005) } \\
\text { (Cederbrant et al., 2003) }\end{array}$ \\
\hline $\begin{array}{l}\text { Adaptive } \\
\text { immunity }\end{array}$ & $\begin{array}{l}\text { T lymphocytes proliferation } \\
\text { Mixed lymphocyte reaction } \\
\text { Cytotoxic T Iymphocyte activity } \\
\text { T cell-dependent antibody } \\
\text { response } \\
\text { Dendritic call maturation }\end{array}$ & not yet & $\begin{array}{c}\text { (Carfi' et al., 2007) } \\
\text { (Elves and Israëls, 1965) } \\
\text { (Dietert et al., 2010a) } \\
\text { (Koeper and Vohr, 2009) } \\
\text { (Dietert et al., 2010b; Toebak et al., } \\
\text { 2008) }\end{array}$ \\
\hline
\end{tabular}

The key targets of different in vitro assays are summarized in TABLE 1. The major advantage of these assays is that all of them can be performed using peripheral blood, which can be obtained and tested easily and repeatedly from the same individual (or animal). However, some general limitations of the in vitro methods still exist for in vitro immunotoxicity testing, such as difficulty in reproducing the integrity of the system, diminishing the cell-cell and organ interaction, and lacking the possibility of metabolism (Lankveld et al., 2010). 


\section{Omics-based approaches for immunotoxicity}

For many immunotoxic compounds, the precise mechanisms of action underlying their toxicity are still not well-understood. The application of "omics" techniques, for instance transcriptomics and proteomics, in the field of immunotoxicology, also referred to as immunotoxicogenomics, appears to be promising to fill this gap, and are increasingly used for this purpose (Luebke et al., 2006). This is of interest for both scientific and nonscientific communities because the generated data can be used for different goals. Scientists would be interested in identifying a broad range of genes that are affected by chemicals and pharmaceuticals, in order to unravel the underlying mechanisms of action, whereas the regulatory communities would be particularly interested in the genes that are indicative of a toxicological end point.

\subsection{Transcriptomics}

The application of transcriptomics in toxicology aims at identification of specific gene expression profiles in biological systems that are associated with chemical exposure. The assumption that the expression pattern of a gene product is tightly related with its function makes gene expression profiling a useful tool in studying the underlying mechanisms of action of toxicants. In addition, it is generally believed that compounds leading to the same (intermediate) endpoint may have similar effects on gene expression, therefore, gene expression profiling may also be helpful in characterization of structural and functional compound classes and identification of biomarkers that can be used for prediction of specific toxic endpoints (Tugwood et al., 2003; Waters and Fostel, 2004).

In short, total RNA is isolated from control and exposed cells or tissues. cDNA is synthesized and can be used in an amplification step to yield fluorescently-labelled cRNA, which will then be hybridized onto microarray slides containing multiple copies of probes representing specific genes. After washing, the arrays will be scanned, which yields intensity for all genes examined. After data normalization and statistics, differentially expressed genes can be identified. Cluster analysis and/ or principle component analysis (PCA) are capable of showing genes with similar expression patterns; and biological interpretation of the transcriptome data can be achieved by pathway analysis (de Longueville et al., 2004).

Besides widely accepted microarray technologies, next-generation sequencing (NGS) is increasingly being used for quantification and characterization of toxicant-induced effects on transcriptomes. Compared to microarray-based approaches, NGS has several advantages, such as the detection of isoform abundance and alternative splicing events (Jayapal, 2012). Moreover, NGS can detect 25\% more genes than can microarray on the basis of known transcripts (Sultan et al., 2008), and produce highly replicable results with little technical variation (Marioni et al., 2008). It has also been found to be more sensitive in detecting genes with low expression levels (Liu et al., 2011; Sîrbu et al., 2012; 
Su et al., 2011). Nevertheless, comparative studies indicated that the differentially expressed genes that were selected under the same criteria were still consistent between the two platforms (Su et al., 2009).

Up till now, most immunotoxicogenomics studies were in vivo experiments. Several studies have shown that different direct immunotoxicants could have overlapping mechanisms of action at the transcriptome level. For instance, Patterson and Germolec, 2006 studied the gene expression changes induced by four immunosuppressants, being 2,3,7,8-tetrachlorodibenzo-p-dioxin (TCDD), diethylstilbestrol (DES), dexamethasone (DEX), and cyclophosphamide (CP) in murine spleen and thymus. Initial analysis of this data set has shown that the genes that were commonly regulated by all compounds were mainly involved in apoptosis, antigen processing and presentation, immune cell activation and lymphocyte proliferation (Patterson and Germolec, 2006). In addition, modulation of the genes involved in T cell receptor (TCR) complex and CD28 signaling pathways, which play crucial roles in lineage commitment and T cell development, was also found for DEX, DES, and CP in the thymus (Frawley et al., 2011). More recently, Baken et al., 2008 performed microarray analysis in murine spleens after in vivo exposure to four immunosuppressants, being bis(tri-n-butyl)tinoxide (TBTO), cyclosporine A ( $\mathrm{Cs} A$ ), benzo[a]pyrene (BaP), and acetominophen (APAP). Cell division was identified as the process that was most significantly affected by all compounds (Baken et al., 2008). Based on the results from these studies, it appears that the immunosuppressive properties of the test compounds were mainly mediated by their effects on cell cycle arrest. Due to the high proliferation rate of immune cells, it could be that immune cells are more sensitive to modulation of cell cycle controlling pathways by toxins than other cell types.

Regarding in vitro studies, the application of immunotoxicogenomics has led to improved mechanistic understanding for a limited number of direct immunotoxicants. For instance, transcriptome analysis of human lymphocytes exposed in vitro to TBTO and the trichothecene mycotoxin deoxynivalenol (DON) has led to the identified of calcium-mediated activation of endoplasmic reticulum (ER) and oxidative stress (Katika et al., 2012a; Katika et al., 2012b; Katika et al., 2011).

Besides its application in mechanistic studies, gene expression profiling has also been applied in identification of biomarkers within the field of direct immunotoxicity. Hochstenbach et al., 2010 studied the transcriptomic changes in human PBMCs in response to 12 compounds. By applying Recursive Feature Elimination based on a Support Vector Machine (SVM-RFE) algorithm, they identified a set of 48 genes whose expression profiling can be used for classification of direct immunotoxicants (Hochstenbach et al., 2010). However, further validation is needed in order to further determine the prediction performance of this gene set.

\subsection{Proteomics}


The application of proteomics in toxicology aims at identification of specific protein expression profiles in biological systems that are associated with chemical exposure. The information obtained from proteomics does not always correlate with transcriptomics. This is caused by the fact that not all mRNAs are translated into proteins due to differences in RNA stability and post-transcriptional processing. Therefore, proteomics has the principle advantage that the measurements focus on the end product of the gene expression cascade, whose information is more closely related to biological functions than the message level (Cox and Mann, 2011). In addition, proteomics can provide additional insights into the mechanisms of action of toxins, and may also allow identification of biomarkers at protein level (Kennedy, 2002).

Currently, mass spectrometry (MS)-based methods are the most commonly applied techniques in proteomics (Han et al., 2008). Before the MS analysis, proteins extracted from biological materials need to be converted into peptides. This can be achieved by two major strategies. The first one involves solubilization of proteins with detergents, separation of proteins by sodium dodecyl sulfate (SDS) polyacrylamide gel electrophoresis (one- or two-dimensional) and digestion of the gel-trapped proteins by trypsin, being the 'in-gel' digestion (Shevchenko et al., 1996). The second one comprises protein extraction with strong chaotropic reagents such as urea and thiourea, followed by protein precipitation and digestion under denaturing conditions, which is the so called 'in-solution' digestion. Then the separation step is normally done with two dimensional peptide separation (Washburn et al., 2001). More recently, Wisniewski et al., 2009 introduced a novel, filter-aided samples preparation (FASP) approach that combined the advantages of both in-gel and in-solution digestion. In short, the sample is first solubilized in SDS, then retained and concentrated in an ultrafiltration device. The filter unit is then used for detergent removal, buffer exchange, chemical modification and protein digestion. After these steps, the peptides are eluted off the filter (Wisniewski et al., 2009).

The use of stable isotopes for sample labelling allows quantitative detection of dynamic changes in protein abundance by MS analysis. The isotope labelling could be performed at organism, cell, protein, or peptide levels, depending on the origin of the sample. During the labelling, isotopes are either incorporated metabolically in cells and small organisms, or postmetabolically in proteins and peptides via chemical or enzymatic reactions (Gevaert et al., 2008).

One of the major challenges for proteomics studies is the extremely broad dynamic range of protein expression, which spans over ten orders of magnitude. However, the sensitivity of MS in complex samples, especially for body fluids, is limited (Solier and Langen, 2014).

A few proteomics studies have focused on the immunotoxic mechanisms of mycotoxin DON. Exposure of the mouse thymoma cell line EL4 to DON resulted in differential expression of 30 proteins. The majority of these proteins were up-regulated and included key metabolic enzymes, chaperones, enzymes implicated in protein folding, and proteins involved in protein degradation. In addition, My-binding protein 1a (MYBBP1A), which is a transcription factor known as a repressor for a number of transcription factors such as PGC-1a, C-myb, and p65 of the NF-kB family, was among the up-regulated proteins by DON (Osman et al., 2010). A proteomics study on human T and B 18 
lymphocyte cell lines also demonstrated that protein degradation process was up-regulated by DON exposure. In addition, several proteins related with regulation of transcription were modulated by DON as well (da Costa et al., 2011).

\subsection{Phosphoproteomics}

The complexity of proteome also lies in the fact that many proteins undergo a wide variety of posttranslational modifications, which regulate the function, localization, complex formation, and degradation of proteins. One type of post-translational modification is phosphorylation, which is common for many enzymes and structural proteins during the cellular signalling transduction process. An alteration in phosphorylation status usually indicates a change in protein activity, which can be an alert that a specific signalling pathway is activated or inhibited (Schmelzle and White, 2006).

Due to the optimization of enrichment methods for phosphoproteins and phosphopeptides, which include application of phosphor-specific antibodies, immobilized metal affinity chromatography (IMAC), and titanium dioxide chromatography, the analysis of the entire phosphoproteome (phosphoproteomics) is feasible. In addition, the use of stable isotope labelling of amino acids in cell culture (SILAC) has emerged as an effective method in which only selected amino acids are labelled (Zanivan et al., 2013).

Phosphoproteomics has been applied in the mechanistic studies of a limited number of immunotoxicants. Phosphoproteomics analysis of spleens from mice exposed to DON for relatively short time periods $(5,15$, and $30 \mathrm{~min})$ identified in total 90 significantly affected phosphoproteins, which are involved in cytoskeleton organization, regulation of apoptosis, and lymphocyte activation and development. A number of affected phosphoproteins are known to play a role in MAPK and PI3K/Akt signaling pathways (Pan et al., 2013). Recently, the immunotoxic effects of TBTO have also been studied at phosphoproteome level. A total of 160 phosphoproteins and 328 phosphorylation sites were identified in the mouse EL4 cell line after TBTO exposure, which are involved in proliferation and energy sensor pathways (Osman and van Loveren, 2012).

\subsection{Novel techniques in (phospho)proteomics}

Besides the traditional separation- and MS-based technologies, application of antibodies as capture and/or detection reagents in high-throughput analyses of proteins has become more and more popular (Liu et al., 2008). A collection of antibodies spotted and fixed on a solid surface such as glass, plastic, or silicone chip, or the so called antibody array, has often been used for detecting protein expression from cell lysates in scientific research and to identify special biomarkers in serum or urine for diagnostic purposes (Liu et al., 2008; Rivas et al., 2008). Compared to the traditional techniques, antibody arrays show good reproducibility, high throughput, high sensitivity, and quantitative accuracy 
over a wide range of concentrations (Chandramouli and Qian, 2009; Haab et al., 2001). In addition, in most of cases, the identities of the measured proteins are already known, which makes it possible to generate and test specific hypothesis and biologically interpret the observed alterations (Haab, 2005).

Another high-content approach that can be used to measure the phosphorylation of protein kinases is the PamChip $®$ microarray technology. These arrays are capable of measuring phosphorylation changes for more than hundred peptides simultaneously. The peptides are immobilized on a porous three-dimensional layer of aluminium oxide with long branched interconnected channels that extend from top to bottom through the material. This structure allows the enzymatic reactions of kinases to be followed kinetically (Hilhorst et al., 2009).

\section{Objectives and outline of the thesis}

Numerous compounds, including metals, mycotoxins, agricultural pesticides, industrial chemicals, and certain drugs, etc., are known to have immunotoxicological properties. Humans are unavoidably exposed to these compounds through food and drinking water consumption and the environment. Therefore, it is of great importance to evaluate the immunotoxic properties of new compounds, and also to study the molecular mechanisms of known immunotoxicants.

Currently, immunotoxicity testings are mainly based on general toxicity studies with in vivo animal models. However, animal testing has several drawbacks, including limited prediction towards the human situation due to interspecies differences, the time and money consuming nature of animal experimentation, and ethical concerns. It has been estimated that for registration of one single pesticide, about 7,000 animals are needed at a cost of tens of millions of dollars in a matter of years (Erickson, 2011). However, on the other hand, more than a thousand new chemicals and pharmaceuticals are produced each year, and all of them are required to be tested according to regulatory frameworks, such as the European chemicals policy, commonly known as REACH (Tetko et al., 2008). Therefore, it is obvious that in vivo tests are not able to evaluate all of them. In addition, in in vivo studies, animals are often exposed to high concentrations of chemicals, which makes it difficult to extrapolate the toxic effect to human because environmental exposure may be 100 or even 1000- fold lower. Taken together, the current situation urgently calls for the development of in vitro alternative testing strategies.

In 2007, the National Research Council (NRC) of the U.S. National Academy of Science published a report entitled "Toxicity testing in the $21^{\text {st }}$ Century: A Vision and A Strategy", advocating a paradigm shift in the field of toxicology. The major changes include the shift from high-dose animal testing to in vitro alternative testing methods, and the shift from apical toxicity endpoints (e.g. tumor formation and death) to the perturbation of toxicologically relevant pathways, also referred to as Adverse Outcome Pathways (AOPs). Successful implementation of this vision will lead to more accurate and humanrelevant risk assessments, an overall increase in the efficiency of toxicology testing and decrease in 
the use of experimental animals (Krewski et al., 2010). In the past decades, high priority has been given to the development of in vitro alternatives for animal testing. Meanwhile, a number of emerging fields and techniques are also contributing in gaining insights into the biological responses to chemicals in human tissues, including the application of genomics techniques in the field of immunotoxicology.

In the light of these new developments and views in toxicity testing, the objectives of this $\mathrm{PhD}$ project were 1) to study the underlying mechanisms of action of direct immunotoxicants by applying -omics based approaches in human in vitro systems, and 2) to identify functional based biomarkers serving as a basis for the development of an in vitro predictive assay to screen chemicals for their immunotoxic properties, thereby contributing to the refinement, reduction and ultimately replacement of current direct immunotoxicity tests on animals.

To reach this aim, we selected the human T lymphoblastic cell line Jurkat as the in vitro model. The rationale behind this choice was that the Jurkat cell line has frequently been used in traditional in vitro studies of the immune system, such as assessment of mitogenic stimuli (Daaka et al., 1996; Fernández-Riejos et al., 2008; Makar et al., 1994). Furthermore, it is of human origin; therefore, no interspecies extrapolation of the data is needed. In addition, the experiments done with cell lines is less labour-intensive and more reproducible as compared to the ones with primary cells. The present thesis is divided into two sections, with Section I focusing on the mechanisms of action of direct immunotoxicants, and Section II dealing with the identification and validation of transcriptomics-based classifiers for direct immunotoxicity. Section I contains in total four chapters. Chapter 2 concerns a wide survey study on the effects of a large variety of compounds (in total 31) including known direct immunotoxicants, chemicals structurally related to the direct immunotoxicants, immunosuppressive drugs with well-characterized molecular targets, and nonimmunotoxic control chemicals. The effects of these test compounds on the transcriptome of Jurkat cells were assessed and allowed to identify several common mechanisms of action for direct immunotoxicity, and assign mechanisms of action to certain immunotoxicants for which mechanistic insights were previously limited or lacking. In Chapter 3, the effects of a few selected direct immunotoxicants, immunosuppressive drugs, and nonimmunotoxic control compounds on protein phosphorylation in Jurkat cells were assessed using a protein antibody array. The results indicated that some cellular signaling pathways were affected by several immunotoxicants. The model immunotoxicant TBTO was chosen to verify the data obtained with the antibody arrays. Comparison of the protein phosphorylation and transcriptome data of Jurkat cells in response to TBTO revealed good correlation at the pathway level and indicated both known and novel mechanisms of action, including the effect of TBTO on chemotaxis. In Chapter 4 , an in vitro functional assay for chemotaxis was set up in order to verify the results obtained in the previous chapter. In order to determine whether the effects of TBTO on the Jurkat cell line would also occur in primary immune cells, human peripheral blood monocytes (PBMCs) were exposed to TBTO and subjected to microarray analysis in Chapter 5. Biological interpretation of the data showed that several biological processes affected by TBTO in Jurkat cells were also affected in PBMCs. Section II contains in total two chapters. Chapter 6 describes the identification and external validation of 
classifiers for direct immunotoxicity. These classifiers were identified on the basis of the transcriptomic data generated in Chapter 2. The results demonstrated that a set of 25 genes can be used to distinguish immunotoxic chemicals and drugs from nonimmunotoxic compounds with high accuracy. The prediction performance of this classifier gene set has been further examined using new classes of immunotoxicants and a larger number of nonimmunotoxic control compounds in Chapter 7. Finally, Chapter 8 summarizes and discusses the results of the present thesis and provides future perspectives on immunotoxicity testing. 


\section{Reference}

Alves, M. F., Fraiji, N. A., Barbosa, A. C., De Lima, D. S., Souza, J. R., Dórea, J. G., and Cordeiro, G. W. O. (2006). Fish consumption, mercury exposure and serum antinuclear antibody in Amazonians. International Journal of Environmental Health Research 16(4), 255-262.

Baken, K. A., Pennings, J. L., Jonker, M. J., Schaap, M. M., de Vries, A., van Steeg, H., Breit, T. M., and van Loveren, H. (2008). Overlapping gene expression profiles of model compounds provide opportunities for immunotoxicity screening. Toxicology and Applied Pharmacology 226(1), 46-59.

Bouvier d'Yvoire, M., Bremer, S., Casati, S., Ceridono, M., Coecke, S., Corvi, R., Eskes, C., Gribaldo, L., Griesinger, C., Knaut, H., Linge, J. P., Roi, A., Zuang, V., Balls, M., Combes, R. D., and Bhogal, N. (2012). ECVAM and new technologies for toxicity testing. In New Technologies for Toxicity Testing (M. Balls, R. D. Combes, and N. Bhogal, Eds.), Vol. 745, pp. 154-180. Springer US.

Carfi', M., Gennari, A., Malerba, I., Corsini, E., Pallardy, M., Pieters, R., Van Loveren, H., Vohr, H. W., Hartung, T., and Gribaldo, L. (2007). In vitro tests to evaluate immunotoxicity: A preliminary study. Toxicology 229(1-2), 11-22.

Cederbrant, K., Marcusson-Ståhl, M., Condevaux, F., and Descotes, J. (2003). NK-cell activity in immunotoxicity drug evaluation. Toxicology 185(3), 241-250.

Chandramouli, K., and Qian, P.-Y. (2009). Proteomics: challenges, techniques and possibilities to overcome biological sample complexity. Human Genomics and Proteomics 1(1).

Chang, K. J., Hsieh, K. H., Lee, T. P., Tang, S. Y., and Tung, T. C. (1981). Immunologic evaluation of patients with polychlorinated biphenyl poisoning: determination of lymphocyte subpopulations. Toxicology and Applied Pharmacology 61(1), 58-63.

Chang, K. J., Hsieh, K. H., Tang, S. Y., Tung, T. C., and Lee, T. P. (1982). Immunologic evaluation of patients with polychlorinated biphenyl poisoning: evaluation of delayed-type skin hypersensitive response and its relation to clinical studies. J Toxicol Environ Health 9(2), 217-23.

Cooper, G. S., Parks, C. G., Treadwell, E. L., St. Clair, E. W., Gilkeson, G. S., and Dooley, M. A. (2004). Occupational risk factors for the development of systemic lupus erythematosus. Journal of Rheumatology 31(10), 1928-1933.

Cox, J., and Mann, M. (2011). Quantitative, high-Resolution proteomics for data-driven systems biology. Annual Review of Biochemistry 80(1), 273-299.

da Costa, A. N., Mijal, R. S., Keen, J. N., Findlay, J. B. C., and Wild, C. P. (2011). Proteomic analysis of the effects of the immunomodulatory mycotoxin deoxynivalenol. Proteomics 11(10), 1903-1914.

Daaka, Y., Friedman, H., and Klein, T. W. (1996). Cannabinoid receptor proteins are increased in Jurkat, human T-cell line after mitogen activation. Journal of Pharmacology and Experimental Therapeutics 276(2), 776-783.

De Jong, W. H., and van Loveren, H. (2007). Screening of xenobiotics for direct immunotoxicity in an animal study. Methods 41(1), 3-8.

de Longueville, F., Bertholet, V., and Remacle, J. (2004). DNA microarray as a tool in toxicogenomics. Comb Chem High Throughput Screen 7(3), 207-11. 
Descotes, J. (2005). Immunotoxicology: role in the safety assessment of drugs. Drug Saf 28(2), 12736.

Dietert, R. R., Burleson, G., Burleson, F., and Dietert, R. (2010a). The cytotoxic T lymphocyte assay for evaluating cell-mediated immune function. In Immunotoxicity Testing (R. R. Dietert, Ed.), Vol. 598, pp. 195-205. Humana Press.

Dietert, R. R., Gao, D., and Lawrence, D. (2010b). Dendritic cells in immunotoxicity testing. In Immunotoxicity Testing (R. R. Dietert, Ed.), Vol. 598, pp. 259-281. Humana Press.

Dobrovolskaia, M. A., and McNeil, S. E. (2013). Understanding the correlation between in vitro and in vivo immunotoxicity tests for nanomedicines. Journal of Controlled Release 172(2), 456-466.

Elves, M., and Israëls, M. C. G. (1965). Lymphocyte transformation in cultures of mixed leucocytes a possible test of histocompatibility. The Lancet 285(7397), 1184-1186.

EPA (1998). Health effects test guidelines, Immunotoxicity, OPPTS 870. 7800.

Erickson, B. E. (2011). Modernizing toxicity tests. Chemical \& Engineering News Archive 89(29), 2526.

FDA (2002). Guidance for industry: immunotoxicology evaluation of investigational new drugs.

Fernández-Riejos, P., Goberna, R., and Sánchez-Margalet, V. (2008). Leptin promotes cell survival and activates Jurkat $T$ lymphocytes by stimulation of mitogen-activated protein kinase. Clinical \& Experimental Immunology 151(3), 505-518.

Frawley, R., White, K., Brown, R., Musgrove, D., Walker, N., and Germolec, D. (2011). Gene expression alterations in immune system pathways in the thymus after exposure to immunosuppressive chemicals. Environ Health Perspect 119(3), 371-376.

Gardner, R. M., Nyland, J. F., Silva, I. A., Maria Ventura, A., Maria de Souza, J., and Silbergeld, E. K. (2010). Mercury exposure, serum antinuclear/antinucleolar antibodies, and serum cytokine levels in mining populations in Amazonian Brazil: a cross-sectional study. Environmental Research 110(4), 345-354.

Gennari, A., Ban, M., Braun, A., Casati, S., Corsini, E., Dastych, J., Descotes, J., Hartung, T., Hooghe-Peters, R., House, R., Pallardy, M., Pieters, R., Reid, L., Tryphonas, H., Tschirhart, E., Tuschl, H., Vandebriel, R., and Gribaldo, L. (2005). The use of in vitro systems for evaluating immunotoxicity: the report and recommendations of an ECVAM workshop. Journal of Immunotoxicology 2(2), 61-83.

Gevaert, K., Impens, F., Ghesquière, B., Van Damme, P., Lambrechts, A., and Vandekerckhove, J. (2008). Stable isotopic labeling in proteomics. Proteomics 8(23-24), 4873-4885.

Haab, B. B. (2005). Antibody arrays in cancer research. Molecular \& Cellular Proteomics 4(4), 377383.

Haab, B. B., Dunham, M. J., and Brown, P. O. (2001). Protein microarrays for highly parallel detection and quantification of specific proteins and antibodies in complex solutions. Genome Biol. 2(2), Research0004-research0004.13.

Haglund, C., Åleskog, A., Håkansson, L. D., Höglund, M., Jacobsson, S., Larsson, R., and Lindhagen, E. (2010). The FMCA-GM assays, high throughput non-clonogenic alternatives to CFU-GM in preclinical hematotoxicity testing. Toxicology Letters 194(3), 102-107. 
Han, X., Aslanian, A., and Yates lii, J. R. (2008). Mass spectrometry for proteomics. Current Opinion in Chemical Biology 12(5), 483-490.

Hilhorst, R., Houkes, L., van den Berg, A., and Ruijtenbeek, R. (2009). Peptide microarrays for detailed, high-throughput substrate identification, kinetic characterization, and inhibition studies on protein kinase A. Analytical Biochemistry 387(2), 150-161.

Hochstenbach, K., van Leeuwen, D. M., Gmuender, H., Stølevik, S. B., Nygaard, U. C., Løvik, M., Granum, B., Namork, E., van Delft, J. H. M., and van Loveren, H. (2010). Transcriptomic profile indicative of immunotoxic exposure: in vitro studies in peripheral blood mononuclear cells. Toxicological Sciences 118(1), 19-30.

Holsapple, M. P. (1995). The plaque-forming cell (PFC) response in immunotoxicology: an approach to monitoring the primary effect function of B lymphocytes. In Methods in Immunotoxicology (J. H. D. G.R. Burleson, and A.E. Munson, Ed.), Vol. 1. Wiley-Liss, New York.

Humphrey, H. (1988). Chemical contaminants in the Great Lakes: the human health aspect. In Toxic Contaminants and Ecosystem Health: A Great Lakes Focus (M. Evans, Ed.), pp. 153-165. John Wiley and Sons, New York.

$\mathrm{ICH}$ (2006). Topic S8, immunotoxicity studies for human pharmaceuticals, CHMP/167235/2004, European Medicine Agencies (EMEA).

Institóris, L., Siroki, O., Dési, I., Lesznyák, J., Serényi, P., Szekeres, É., and Petri, I. (1998). Extension of the protocol of OECD guideline 407 (28-day repeated dose oral toxicity test in the rat) to detect potential immunotoxicity of chemicals. Human \& Experimental Toxicology 17(4), 206-211.

International Programme on Chemical Safety (IPCS) (1996). Principles and methods for assessing direct immunotoxicity associated with exposure to chemicals, environmental health criteria 180 . World Health Organization, Geneva.

Janeway, C. A., Jr. Travers, P., Walport, M., and Shlomchik, M. J. (2001). Immunology, 5th edition. (C. A. Janeway, Ed.), Garland Science, New York.

Jayapal, M. (2012). Integration of next-generation sequencing based multi-omics approaches in toxicogenomics. Front. Genet 3: 88.

Karakaya, A., Ates, I., and Yucesoy, B. (2004). Effects of occupational polycyclic aromatic hydrocarbon exposure on T-lymphocyte functions and natural killer cell activity in asphalt and coke oven workers. Human \& Experimental Toxicology 23(7), 317-322.

Katika, M. R., Hendriksen, P. J., de Ruijter, N. C., van Loveren, H., and Peijnenburg, A. (2012a). Immunocytological and biochemical analysis of the mode of action of bis (tri-n-butyltin) tri-oxide (TBTO) in Jurkat cells. Toxicology Letters 212(2), 126-136.

Katika, M. R., Hendriksen, P. J., Shao, J., van Loveren, H., and Peijnenburg, A. (2012b). Transcriptome analysis of the human $\mathrm{T}$ lymphocyte cell line Jurkat and human peripheral blood mononuclear cells exposed to deoxynivalenol (DON): New mechanistic insights. Toxicology and Applied Pharmacology 264(1), 51-64.

Katika, M. R., Hendriksen, P. J., van Loveren, H., and Peijnenburg, A. (2011). Exposure of Jurkat cells to bis (tri-n-butyltin) oxide (TBTO) induces transcriptomics changes indicative for ER- and 
oxidative stress, T cell activation and apoptosis. Toxicology and Applied Pharmacology 254(3), 311322.

Kennedy, S. (2002). The role of proteomics in toxicology: identification of biomarkers of toxicity by protein expression analysis. Biomarkers 7(4), 269-290.

Kim, G. G., Donnenberg, V. S., Donnenberg, A. D., Gooding, W., and Whiteside, T. L. (2007). A novel multiparametric flow cytometry-based cytotoxicity assay simultaneously immunophenotypes effector cells: Comparisons to a $4 \mathrm{~h}$ 51Cr-release assay. Journal of Immunological Methods 325(1-2), 51-66.

Koeper, L. M., and Vohr, H. W. (2009). Functional assays are mandatory for a correct prediction of immunotoxic properties of compounds in vitro. Food and Chemical Toxicology 47(1), 110-118.

Kolaczkowska, E., and Kubes, P. (2013). Neutrophil recruitment and function in health and inflammation 13(3), 159-175.

Krewski, D., Acosta, D., Andersen, M., Anderson, H., Bailar, J. C., Boekelheide, K., Brent, R., Charnley, G., Cheung, V. G., Green, S., Kelsey, K. T., Kerkvliet, N. I., Li, A. A., McCray, L., Meyer, O., Patterson, R. D., Pennie, W., Scala, R. A., Solomon, G. M., Stephens, M., Yager, J., Zeise, L., Staff of Committee on Toxicity, T., and Assessment of Environmental, A. (2010). Toxicity Testing in the 21st Century: A Vision and a Strategy. Journal of Toxicology and Environmental Health, Part B 13(2-4), 51-138.

Kuratsune, M., Yoshimura, T., Matsuzaka, J., and Yamaguchi, A. (1972). Epidemiologic study on Yusho, a Poisoning Caused by Ingestion of Rice Oil Contaminated with a Commercial Brand of Polychlorinated Biphenyls. Environ Health Perspect 1, 119-128.

Ladics, G. S. (2007). Use of SRBC antibody responses for immunotoxicity testing. Methods 41(1), 919.

Langezaal, I., Hoffmann, S., Hartung, T., and Coecke, S. (2002). Evaluation and prevalidation of an immunotoxicity test based on human whole-blood cytokine release. Altern Lab Anim 30(6), 581-95.

Lankveld, D. P. K., Loveren, H., Baken, K. A., Vandebriel, R. J., and Dietert, R. R. (2010). In vitro testing for direct immunotoxicity: state of the art immunotoxicity testing. In Immunotoxicity Testing (J. M. Walker, Ed.), Vol. 598, pp. 401-423. Humana Press.

Liu, B. S., Ehrlich, J., and Chaga, G. (2008). Antibody arrays for determination of relative protein abundances. In Tissue Proteomics (B. S. Liu, and J. Ehrlich, Eds.), Vol. 441, pp. 129-151. Humana Press.

Liu, S., Lin, L., Jiang, P., Wang, D., and Xing, Y. (2011). A comparison of RNA-Seq and high-density exon array for detecting differential gene expression between closely related species. Nucleic Acids Research 39(2), 578-588.

Lü, Y., and $\mathrm{Wu}, \mathrm{Y}$. (1985). Clinical findings and immunological abnormalities in Yu-cheng patients. Environ Health Perspect 59, 17-29.

Luebke, R. W., Holsapple, M. P., Ladics, G. S., Luster, M. I., Selgrade, M., Smialowicz, R. J., Woolhiser, M. R., and Germolec, D. R. (2006). Immunotoxicogenomics: the potential of genomics technology in the immunotoxicity risk assessment process. Toxicological Sciences 94(1), 22-27.

Luster, M. I., Munson, A. E., Thomas, P. T., Holsapple, M. P., Fenters, J. D., White, K. L., Lauer, L. D., Germolec, D. R., Rosenthal, G. J., and Dean, J. H. (1988). Development of a testing battery to 
assess chemical-induced immunotoxicity: national toxicology program's guidelines for immunotoxicity evaluation in mice. Toxicological Sciences 10(1), 2-19.

Luster, M. I., Portier, C., Pait, D. G., Rosenthal, G. J., Germolec, D. R., Corsini, E., Blaylock, B., Pollock, P., Kouchi, Y., Craiq, W., White, D., Munson, A., and Comment, C. (1993). Risk assessment in immunotoxicology. II. Relationships between immune and host resistance tests. Fundam Appl Toxicol 21(1), 71-82.

Luster, M. I., Portier, C., Pait, D. G., White, K. L., Gennings, C., Munson, A. E., and Rosenthal, G. J. (1992). Risk assessment in immunotoxicology: I. Sensitivity and predictability of immune tests. Toxicological Sciences 18(2), 200-210.

Makar, R. S., Lipsky, P. E., and Cuthbert, J. A. (1994). Non-sterol regulation of low density lipoprotein receptor gene expression in T cells. Journal of Lipid Research 35(10), 1888-95.

Marioni, J. C., Mason, C. E., Mane, S. M., Stephens, M., and Gilad, Y. (2008). RNA-seq: An assessment of technical reproducibility and comparison with gene expression arrays. Genome Research 18(9), 1509-1517.

Mauri, C., and Bosma, A. (2012). Immune regulatory function of B cells. Annual Review of Immunology 30(1), 221-241.

Nyland, J. F., Wang, S. B., Shirley, D. L., Santos, E. O., Ventura, A. M., de Souza, J. M., and Silbergeld, E. K. (2011). Fetal and maternal immune responses to methylmercury exposure: A crosssectional study. Environmental Research 111(4), 584-589.

OECD (2008). Repeated dose 28-day oral toxicity study in rodents. OECD Guidelines for Chemical Testing, TG 407: http://iccvam.niehs.nih.gov/SuppDocs/FedDocs/OECD/OECDtg407-2008.pdf.

Osman, A. M., Pennings, J. L. A., Blokland, M., Peijnenburg, A., and van Loveren, H. (2010). Protein expression profiling of mouse thymoma cells upon exposure to the trichothecene deoxynivalenol (DON): Implications for its mechanism of action. Journal of Immunotoxicology 7(3), 147-156.

Osman, A. M., and van Loveren, H. (2012). Phosphoproteomic analysis of mouse thymoma cells treated with tributyltin oxide: TBTO affects proliferation and energy sensing pathways. Toxicological Sciences 126(1), 84-100.

Oughton, J. A., and Kerkvliet, N. I. (2005). Immune cell phenotyping using flow cytometry. Current Protocols in Toxicology 23(18.8), 18.8.1-18.8.24.

Pan, X., Whitten, D. A., Wu, M., Chan, C., Wilkerson, C. G., and Pestka, J. J. (2013). Early phosphoproteomic changes in the mouse spleen during deoxynivalenol-induced ribotoxic stress. Toxicological Sciences 135(1), 129-143.

Parkin, J., and Cohen, B. (2001). An overview of the immune system. The Lancet 357(9270), 17771789.

Patterson, R. M., and Germolec, D. R. (2006). Gene expression alterations in immune system pathways following exposure to immunosuppressive chemicals. Annals of the New York Academy of Sciences 1076(1), 718-727.

Pessina, A., Albella, B., Bayo, M., Bueren, J., Brantom, P., Casati, S., Croera, C., Gagliardi, G., Foti, P., Parchment, R., Parent-Massin, D., Schoeters, G., Sibiril, Y., van Den Heuvel, R., and Gribaldo, L. (2003). Application of the CFU-GM assay to predict acute drug-induced neutropenia: an international 
blind trial to validate a prediction model for the maximum tolerated dose (MTD) of myelosuppressive xenobiotics. Toxicological Sciences 75(2), 355-367.

Pessina, A., Albella, B., Bueren, J., Brantom, P., Casati, S., Gribaldo, L., Croera, C., Gagliardi, G., Foti, P., Parchment, R., Parent-Massin, D., Sibiril, Y., and van Den Heuvel, R. (2001). Prevalidation of a model for predicting acute neutropenia by colony forming unit granulocyte/macrophage (CFU-GM) assay. Toxicology in Vitro 15(6), 729-740.

Rich, I. N., and Hall, K. M. (2005). Validation and development of a predictive paradigm for hemotoxicology using a multifunctional bioluminescence colony-forming proliferation assay. Toxicological Sciences 87(2), 427-441.

Ringerike, T., Ullerås, E., Völker, R., Verlaan, B., Eikeset, Å., Trzaska, D., Adamczewska, V., Olszewski, M., Walczak-Drzewiecka, A., Arkusz, J., van Loveren, H., Nilsson, G., Lovik, M., Dastych, J., and Vandebriel, R. J. (2005). Detection of immunotoxicity using T-cell based cytokine reporter cell lines (“Cell Chip"). Toxicology 206(2), 257-272.

Rivas, L. A., García-Villadangos, M., Moreno-Paz, M., Cruz-Gil, P., Gómez-Elvira, J., and Parro, V. c. (2008). A 200-antibody microarray biochip for environmental monitoring: searching for universal microbial biomarkers through immunoprofiling. Analytical Chemistry 80(21), 7970-7979.

Schmelzle, K., and White, F. M. (2006). Phosphoproteomic approaches to elucidate cellular signaling networks. Current Opinion in Biotechnology 17(4), 406-414.

Schultz, K. T., and Grieder, F. (1987). Structure and function of the immune system. Toxicologic Pathology 15(3), 262-264.

Schwenk, M., Klein, R., and Templeton, D. M. (2009). Immunological effects of mercury. Pure Appl. Chem. 81(1), 153-167.

Shevchenko, A., Wilm, M., Vorm, O., and Mann, M. (1996). Mass spectrometric sequencing of proteins from silver-stained polyacrylamide gels. Analytical Chemistry 68(5), 850-858.

Silva, I., Nyland, J., Gorman, A., Perisse, A., Ventura, A., Santos, E., de Souza, J., Burek, C., Rose, N., and Silbergeld, E. (2004). Mercury exposure, malaria, and serum antinuclear/antinucleolar antibodies in amazon populations in Brazil: a cross-sectional study. Environmental Health: A Global Access Science Source 3(1), 11.

Sîrbu, A., Kerr, G., Crane, M., and Ruskin, H. J. (2012). RNA-Seq vs dual- and single-channel microarray data: sensitivity analysis for differential expression and clustering. PLOS ONE 7(12), e50986.

Solier, C., and Langen, H. (2014). Antibody-based proteomics and biomarker research—current status and limitations. Proteomics 14(6), 774-783.

Stewart, C. C., Cookfair, D. L., Hovey, K. M., Wende, K. E., Bell, D. S., and Warner, C. L. (2003). Predictive immunophenotypes: Disease-related profile in chronic fatigue syndrome. Cytometry 53B(1), 26-33.

Stølevik, S. B., Nygaard, U. C., Namork, E., Haugen, M., Kvalem, H. E., Meltzer, H. M., Alexander, J., van Delft, J. H. M., van Loveren, H., Løvik, M., and Granum, B. (2011). Prenatal exposure to polychlorinated biphenyls and dioxins is associated with increased risk of wheeze and infections in infants. Food and Chemical Toxicology 49(8), 1843-1848. 
Stølevik, S. B., Nygaard, U. C., Namork, E., Haugen, M., Meltzer, H. M., Alexander, J., Knutsen, H. K., Aaberge, I., Vainio, K., van Loveren, H., Løvik, M., and Granum, B. (2013). Prenatal exposure to polychlorinated biphenyls and dioxins from the maternal diet may be associated with immunosuppressive effects that persist into early childhood. Food and Chemical Toxicology 51(0), 165-172.

Su, Z., Li, Z., Chen, T., Li, Q.-Z., Fang, H., Ding, D., Ge, W., Ning, B., Hong, H., Perkins, R. G., Tong, W., and Shi, L. (2011). Comparing next-generation sequencing and microarray technologies in a toxicological study of the effects of aristolochic acid on rat kidneys. Chemical Research in Toxicology 24(9), 1486-1493.

Su, Z., Ning, B., Fang, H., Hong, H., Perkins, R., Tong, W., and Shi, L. (2009). Next-generation sequencing: A revolutionary tool for toxicogenomics. In General, Applied and Systems Toxicology (B. Ballantyne, T. Marrs, and T. Syversen, Eds.). John Wiley \& Sons, Ltd.

Sultan, M., Schulz, M. H., Richard, H., Magen, A., Klingenhoff, A., Scherf, M., Seifert, M., Borodina, T., Soldatov, A., Parkhomchuk, D., Schmidt, D., O'Keeffe, S., Haas, S., Vingron, M., Lehrach, H., and Yaspo, M.-L. (2008). A global view of gene activity and alternative splicing by deep sequencing of the human transcriptome. Science 321(5891), 956-960.

Swain, S., Bradley, L., Croft, M., Tonkonogy, S., Atkins, G., Weinberg, A., Duncan, D., Hedrick, S., Dutton, R., and Huston, G. (1991). Helper T-cell subsets: phenotype, function and the role of lymphokines in regulating their development. Immunol Rev. 123, 115-44.

Tetko, I. V., Sushko, I., Pandey, A. K., Zhu, H., Tropsha, A., Papa, E., Öberg, T., Todeschini, R., Fourches, D., and Varnek, A. (2008). Critical assessment of QSAR models of environmental toxicity against tetrahymena pyriformis: Focusing on applicability domain and overfitting by variable selection. Journal of Chemical Information and Modeling 48(9), 1733-1746.

Toebak, M. J., De Rooij, J., Moed, H., Stoof, T. J., von Blomberg, B. M. E., Bruynzeel, D. P., Scheper, R. J., Gibbs, S., and Rustemeyer, T. (2008). Differential suppression of dendritic cell cytokine production by anti-inflammatory drugs. British Journal of Dermatology 158(2), 225-233.

Tugwood, J. D., Hollins, L. E., and Cockerill, M. J. (2003). Genomics and the search for novel biomarkers in toxicology. Biomarkers 8(2), 79-92.

Turley, S. J., Fletcher, A. L., and Elpek, K. G. (2010). The stromal and haematopoietic antigenpresenting cells that reside in secondary lymphoid organs. Nature Reviews Immunology 10(12), 813825.

Vignali, D. A., Collison, L. W., and Workman, C. J. (2008). How regulatory T cells work 8(7), 523-532.

Washburn, M. P., Wolters, D., and Yates, J. R. (2001). Large-scale analysis of the yeast proteome by multidimensional protein identification technology. Nat Biotech 19(3), 242-247.

Waters, M. D., and Fostel, J. M. (2004). Toxicogenomics and systems toxicology: aims and prospects 5(12), 936-948.

Wisniewski, J. R., Zougman, A., Nagaraj, N., and Mann, M. (2009). Universal sample preparation method for proteome analysis. Nat Meth 6(5), 359-362.

Zanivan, S., Meves, A., Behrendt, K., Schoof, Erwin M., Neilson, Lisa J., Cox, J., Tang, Hao R., Kalna, G., van Ree, Janine H., van Deursen, Jan M., Trempus, Carol S., Machesky, Laura M., 
Linding, R., Wickström, Sara A., Fässler, R., and Mann, M. (2013). In vivo SILAC-based proteomics reveals phosphoproteome changes during mouse skin carcinogenesis. Cell Reports 3(2), 552-566. 


\section{SECTION I}

Potential mechanisms of action underlying chemicalinduced direct immunotoxicity 



\title{
CHAPTER 2
}

\section{Toxicogenomics-based identification of mechanisms for direct immunotoxicity}

\author{
Jia Shao, Madhumohan R. Katika, Peter C. J. Schmeits, Peter J. M. Hendriksen, \\ Henk van Loveren, Ad A. C. M. Peijnenburg, and Oscar L. Volger
}

Toxicol. Sci. 2013, 135 (2): 328-346

Supplementary material can be found at: http://toxsci.oxfordjournals.org/content/early/2013/06/29/toxsci.kft151/suppl/DC1 


\begin{abstract}
Compounds with direct immunotoxic properties, including metals, mycotoxins, agricultural pesticides and industrial chemicals, form potential human health risks due to exposure through food, drinking water, and the environment. Insights into the mechanisms of action are currently lacking for the majority of these direct immunotoxicants. Therefore, the present work aimed to gain insights into the molecular mechanisms underlying direct immunotoxicity. To this end, we assessed in vitro the effects of 31 test compounds on the transcriptome of the human Jurkat $T$ cell line. These compounds included direct immunotoxicants, immunosuppressive drugs with different mode of actions, and nonimmunotoxic control chemicals. Pathway analysis of the microarray data allowed us to identify canonical pathways and Gene Ontology processes that were transcriptionally regulated in common by immunotoxicants (1) with structural similarities, such as the tributyltin chloride and tributyltin oxide that activated the retinoic acid/X receptor signaling pathway, and (2) without structural similarities, such as $\mathrm{As}_{2} \mathrm{O}_{3}$, dibutyltin chloride, diazinon, $\mathrm{MeHg}$, ochratoxin A (OTA), S9-treated OTA, S9-treated cyclophosphamide, and S9-treated benzo[a]pyrene, which activated unfolded protein response, and FTY720, lindane, and propanil, which activated the cholesterol biosynthesis pathway. In addition, processes uniquely affected by individual immunotoxicants were identified, such as the induction of Notch receptor signaling and the downregulation of acute-phase response genes by OTA. These findings were validated by quantitative real-time PCR analysis of genes involved in these processes. Our study indicated that diverse modes of action are involved in direct immunotoxicity and that a set of pathways or genes, rather than one single gene, can be used to screen compounds for direct immunotoxicity.
\end{abstract}




\section{Introduction}

Direct immunotoxicity is defined as direct deleterious effects of a xenobiotic on the functioning of the immune system, whereas indirect immunotoxicity is an allergic reaction, which causes tissue damage upon exposure to a xenobiotic. Direct immunotoxicity can be elicited by either suppression or by activation of the immune system, better known as immunosuppression and immune enhancement, respectively (Corsini and Roggen, 2009; Descotes, 2005; Galbiati et al., 2010). Numerous compounds with direct immunotoxic properties form potential human health risks due to exposure through food (Borchers et al., 2010), drinking water, and the environment (Coelho et al., 2012; Corsini et al., 2012a; Veraldi et al., 2006). Direct immunotoxicants include metals (Tchounwou et al., 2003), mycotoxins (Milićević et al., 2010), agricultural pesticides (Corsini et al. 2012b), industrial chemicals (Veraldi et al., 2006), and certain drugs (Descotes, 2005). Currently, insights into the molecular mechanisms of direct immunotoxicity are lacking for the majority of these compounds.

During the registration and approval of chemicals and drugs, the in vivo identification of direct immunotoxicity is based on gross pathology and haematological parameters in rodent-based animal models (Food and Drug Administration, 2006; Institoris et al., 1998). After the identification of direct immunotoxicity, mechanistic information of direct immunotoxicity at the cellular level is mostly obtained by applying classical assays for immune cell functionality. These assays mainly determine the effects of the xenobiotics on the proliferation, cytokine release, or antibody production of immune cells in vitro upon antigenic, mitogenic or viral stimulation (Lankveld et al., 2010). However, experiments to characterize the effects at the molecular level are generally lacking. In the last decade, (immuno)toxicogenomics, the application of genomics techniques in (immuno)toxicology, has rapidly become a new promising approach, which can be employed to get insight into the molecular mechanisms of (immuno)toxic compounds (Luebke et al., 2006).

Recently, the application of genomics techniques has led to improved mechanistic understanding for a limited set of direct immunotoxic compounds. The rodent in vivo studies by Baken et al., (2008) and Frawley et al., (2011) showed that different direct immunotoxicants could have overlapping mechanisms of action at the level of gene expression: cell cycle arrest was elicited by the polycyclic aromatic hydrocarbon benzo[a]pyrene $(\mathrm{BaP})$, the immunosuppressive drug cyclosporin $\mathrm{A}$ (CsA; calcineurin inhibitor), and the industrial chemical tributyltin oxide (TBTO; organotin) in murine spleens (Baken et al., 2008), whereas T-cell receptor and CD28 signaling were altered in murine thymocytes by the immunosuppressive drugs cyclophosphamide (CP; alkylating agent), diethylstilbestrol (estrogen), and dexamethasone (glucocorticoid) (Frawley et al., 2011). The application of immunotoxicogenomics in in vitro studies has led to improved insights into the modes of action (MOAs) of direct immunotoxic compounds. For instance, transcriptome analyses of human lymphocytes exposed in vitro to immunotoxicants have led to the identification of calcium-mediated induction of endoplasmic reticulum (ER) and oxidative stress by the organotin compound TBTO (Katika et al., 2011, 2012a), and the trichothecene mycotoxin deoxynivalenol (DON) (Katika et al., 2012b). 
Currently, a wide survey study on the potential MOAs of direct immunotoxicants is lacking. Therefore, the present study aims to fill this knowledge gap by assessing the effects of a large variety of compounds on the transcriptome of the human $\mathrm{T}$ cell in vitro. These compounds include chemicals that are known to cause direct immunotoxicity, chemicals structurally related to the direct immunotoxicants, nonimmunotoxic control chemicals, and immunosuppressive drugs with wellcharacterized MOAs. We have chosen to use the Jurkat $\mathrm{T}$ cell line for this in vitro transcriptome profiling study because Jurkat cells are of human origin and are easy to culture. In the present study, Jurkat $T$ cells were exposed to subcytototoxic concentrations of the test compounds in order to identify primary MOAs of the immunotoxicants rather than less specific cell death-related gene expression signatures. The exposure was done for a relatively short time period of $6 \mathrm{~h}$, in order to identify the initial pathways involved in direct immunotoxicity. Subsequently, the exposed cells were subjected to transcriptome analysis. This approach enabled us to identify several common MOAs for direct immunotoxicity and to assign MOAs to several immunotoxicants for which mechanistic insights were previously limited or lacking.

\section{Materials and Methods}

Cell culture. The human lymphoblastic T-cell line (Jurkat) was obtained from the American Type Culture Collection. The Jurkat cells were grown in RPMI-1640 medium supplemented with $10 \%$ heat inactivated fetal calf serum, $2 \mathrm{mM}$ glutamine, $1 \mathrm{mM}$ sodium pyruvate, $1 \mathrm{mM}$ non-essential amino acids, $100 \mathrm{U} / \mathrm{ml}$ penicillin, and $100 \mu \mathrm{g} / \mathrm{ml}$ streptomycin. Cells were cultured at $37^{\circ} \mathrm{C}$ with $5 \% \mathrm{CO}_{2}$ in a humidified atmosphere. The medium was refreshed every 2 days.

Compound selection. The direct immunotoxicants were selected on the basis of their severity of toxicity and environmental abundance (Table 1 and Supplementary table 1). The total number of compounds included in the present study is 31 . Besides environmental contaminants, we also incorporated a group of immunosuppressive drugs on the basis of their well-defined molecular targets (Table 1). The MOAs of these immunosuppressive drugs include DNA alkylation, glucocorticoid receptor activation, inhibition of purine synthesis, calcineurin, mammalian target of rapamycin (mTOR), and the sphingosine-1-phosphate receptor 1 (S1PR). All chemicals tested were purchased from Sigma-Aldrich (Zwijndrecht, The Netherlands), except mono-2-ethylhexyl phthalate (MEHP; Wako chemicals, Neuss, Germany) and fingolimod (FTY720; Selleck Chemicals, via Bio-Connect Diagnostics, Huissen, The Netherlands). Stock solutions of all chemicals were made by dissolving the substances in dimethyl sulfoxide (DMSO; Merck, Darmstadt, Germany).

Metabolic activation system. BaP, OTA, and CP were subjected to in vitro metabolic activation using a human liver S9 fraction (BD Bioscience, Breda, The Netherlands). The S9 reaction mixtures, having a 
total volume of $1 \mathrm{ml}$, consisted of $570 \mu \mathrm{l} \mathrm{H}_{2} \mathrm{O}$ (MQ), $200 \mu \mathrm{l} 0.5 \mathrm{M}$ potassium phosphate buffer ( $\mathrm{pH} 7.4$ ), $100 \mu \mathrm{l}$ NADPH regeneration system solution A (BD Bioscience), $20 \mu \mathrm{l}$ NADPH regeneration system solution B (BD Bioscience), $10 \mu \mathrm{l}$ compound stock in DMSO, and $100 \mu \mathrm{l}$ S9 mix (BD Bioscience). After incubating for respectively 1,6 , and $24 \mathrm{~h}$, the $\mathrm{S} 9$ chemical reaction mixtures were heat inactivated $\left(5 \mathrm{~min}\right.$ at $\left.56^{\circ} \mathrm{C}\right)$ and pooled at equal volumes.

\section{Viability and Cytotoxicity Assays}

WST-1 assay. WST-1 (4-[3-(4-iodophenyl)-2-(4-nitrophenyl)-2H-5-tetrazolio]-1,3-benzene disulfonate, Roche Diagnostic Ned BV, Almere, The Netherlands) is a water soluble tetrazolium salt, which is converted by mitochondrial dehydrogenases to a colored formazan complex. The amount of formazan correlates to the viability of the cells. Twenty hours before exposure, Jurkat cells were plated in advance in 96 well plates resulting in about 20,000 cells per well at the starting point of exposure. Exposure was done in triplicate in $100 \mu \mathrm{l}$ medium for $24 \mathrm{~h}$ to increasing concentrations of the compounds or to the vehicle controls. In the last $2 \mathrm{~h}$ of exposure, $10 \mu \mathrm{l}$ of WST-1 reagent was added. Absorbance was measured at $450 \mathrm{~nm}$ in a microplate reader (BioTek, Winooski, VT).

ATPlite assay. ATPlite assay (Perkin Elmer, Oosterhout, The Netherlands) is based on the production of light caused by the reaction of ATP with luciferase and D-luciferin. The emitted light is proportional to the amount of ATP, which is a marker for cell viability. Jurkat cells were plated as described above for the WST-1 assay. Exposure was done in triplicate in $100 \mu \mathrm{l}$ medium in 96-well plates for $24 \mathrm{~h}$ to increasing concentrations of compounds or to the vehicle controls. After exposure, the assay was performed according the manufacturer's protocol.

ToxiLight assay. ToxiLight assay (Lonza, Breda, The Netherlands) is luminescent cytotoxicity assay based on the release of adenylate kinase (AK) from damaged cells. Jurkat cells were plated as described above for the WST-1 assay. Exposure was done in triplicate in $100 \mu$ medium in 96-well plates for $24 \mathrm{~h}$ to compounds or to the vehicle controls. After exposure, the assay was performed according the manufacturer's protocol.

Chemical exposures. Jurkat cells (passage number 16-20) were seeded in $2.7 \mathrm{ml}$ medium per well in 6 -well plates (750,000 cells/well). After growing the cells for $20 \mathrm{~h}$, exposure was initiated by adding 0.3 $\mathrm{ml}$ medium containing non-cytotoxic concentration of the compounds or vehicle controls. Subsequently, cells were exposed to the compounds for $6 \mathrm{~h}$. The final DMSO concentration in the medium was $0.1 \%$ ( $\mathrm{vol} / \mathrm{vol})$ for all the samples. Each exposure was performed in quadruplicate at four different days. 
RNA isolation and quality control. After exposure, the culture medium was removed after the centrifugation of the cell suspension $\left(5 \mathrm{~min}\right.$ at $300 \mathrm{~g}, 4^{\circ} \mathrm{C}$ ). The resulting cell pellet was homogenized in $600 \mu \mathrm{l}$ red cell lysis buffer (Qiagen, Venlo, The Netherlands) supplemented with $10 \% \beta$ mercaptoethanol and stored at $-80^{\circ} \mathrm{C}$ until further processing. RNA was isolated with the Qiagen QIAshredder kit according to the manufacturer's protocol. Subsequently, RNA was purified using the miRNeasy Mini kit (Qiagen) according to the manufacturer's protocol. RNA yield was assessed spectrophotometrically (NanoDrop 2000, Isogen Life Science, De Meern, The Netherlands). RNA quality was determined by automated gel electrophoresis using Bio-Rad's Experion system (Veenendaal, The Netherlands). Samples with RNA Quality Index numbers $>8$ were considered to be of sufficient quality.

Microarray hybridization and data normalization. Gene expression profiling was performed using Affymetrix U133 Plus 2.0 Arrays (Santa Clara, CA). For each sample, complementary DNA (cDNA) was synthesized from $5 \mu \mathrm{g}$ of total RNA. cDNA synthesis and subsequent synthesis of biotin-labeled cRNA was performed using GeneChip One-Cycle Target Labeling and Control Reagents including the One-Cycle cDNA Synthesis Kit, Poly-A RNA Control Kit, Sample Cleanup Module, IVT Labeling Kit, and Hybridization Control Kit (all from Affymetrix) according to the manufacturer's protocol. Yields of cRNA were quantified spectrophotometrically (Nanodrop). cRNA integrity was assessed using the Bioanalyzer (Agilent Technologies, Amstelveen, The Netherlands). Upon fragmentation, $12 \mu \mathrm{g}$ of cRNAs were hybridized to the arrays for $16 \mathrm{~h}$ at $45^{\circ} \mathrm{C}$ (GeneChip Hybridization Oven 640; Affymetrix). After washing and staining (GeneChip hybridization station, Fluidics Station 450, Affymetrix), the chips were scanned (Affymetrix GeneChip Scanner 3000 7G). Raw data were extracted using GeneChip Operating Software (Affymetrix).

In order to filter out probes with sub-optimal specificity for the encoding genes, custom CDF files were generated from the raw data files, by using the $R$ package available at http://brainarray.mbni.med.umich.edu/Brainarray/Database/CustomCDF/14.1.0/entrezg.asp. Then robust multichip average normalization was applied to the complete data set (Bioconductor). Bioconductor packages were used for the quality control of the microarray data (www.arrayanalysis.org). Boxplots of log-intensity distribution before and after normalization were constructed for between-array comparison (Supplementary fig. 1).

\section{Microarray Data Analyses}

Hierarchical clustering. Unsupervised hierarchical clustering was performed with the publicly available programs Cluster (uncentered correlation; average linkage clustering) and Treeview (Eisen et al., 1998). 
Metacore. Functional interpretation of differentially expressed genes for each chemical was done using Metacore software (GeneGo, St. Joseph, MI). Metacore uses hypergeometric distribution to assess significances for overrepresentation of affected genes in signaling and metabolic pathways (Ekins et al., 2006). For each compound, the affected genes were analyzed with Metacore, separately for the up- and downregulated genes. Pathways with a $p$ value $<0.01$ were considered as significantly regulated. Pathways that were determined by less than four genes or affected by less than two compounds were left out for further analysis (see Supplementary table 4 for the resulting pathway list). Then, pathways irrelevant for the present study (for instance, regress of virus within host cell, response to biotic stimulus, etc.) or irrelevant for the immune system (for instance, brain development, nervous system development, etc.) were manually removed. The $p$ values were then converted into $Z$ values to enable clustering. Pathways obtained a positive or negative $Z$ value when up- or downregulated, respectively. Hierarchical clustering was done as described above.

Gene set enrichment analysis. Gene set enrichment analysis (GSEA) was used to detect the differential expression of biologically relevant gene sets and to provide insight into the affected molecular mechanisms. GSEA makes use of predefined gene sets that are based on previous experimental results and literature. It ranks all genes on the basis of their expression ratios and then calculates whether a particular gene set is significantly enriched at the top or the bottom of the ranking list (Subramanian et al., 2005). Therefore, GSEA has the advantage over other statistical tools that no initial filtering is applied to the data set to select significantly differentially expressed genes. We used the gene sets downloaded from the Gene Ontology (GO) consortium (http://www.geneontology.org/) including biological process, cellular component, and molecular function. Gene sets with a $p$ value $<0.01$ and a false discovery rate value $<0.25$ were considered as significantly regulated. Up- and downregulation of significant gene sets were visualized with heap maps. The $p$ values were converted into $Z$ values to enable clustering. Gene sets obtained a positive or negative $Z$ values when up- or downregulated, respectively. Hierarchical clustering was done as described above.

Quantitative real-time $P C R$ verification. Verification of the microarray outcome was performed at ServiceXS (ServiceXS BV, Leiden, The Netherlands) using the 96.96 BioMark Dynamic Array for realtime PCR (Fluidigm Corporation, San Francisco, CA), according to the manufacturer's instructions. cDNA samples were synthesized from the same RNA samples as used for the microarray experiment using miScript Reverse Transcription kit according to the manufacturer's protocol (Qiagen). Before use on the BioMark array, the cDNA was first subjected to 14 cycles of Specific Target Amplification using a $0.2 x$ mixture of all TaqMan Gene Expression assays in combination with the TaqMan PreAmp Master Mix (Applied Biosystems), followed by fivefold dilution. Thermal cycling and real-time imaging of the BioMark array was done on the BioMark instrument, and $\mathrm{Ct}$ values were extracted using the BioMark real-time PCR analysis software, and $\triangle \Delta C T$ values were used to determine compound effects on mRNA expression levels. 


\section{Results}

\section{Selection of Test Compounds}

We selected 31 test compounds for assessing their effects on the transcriptome of the human Jurkat $\mathrm{T}$ cell line in vitro. These test compounds comprise direct immunotoxic chemicals, immunosuppressive drugs with known MOAs, compounds structurally related to the immunomodulating compounds, and nonimmunotoxic control compounds (Table 1). BaP, OTA, and $\mathrm{CP}$ were bioactivated using human liver S9 fraction. For these three compounds, both the parent compounds and the bioactivated samples were included in the study.

\section{Determination of Exposure Concentrations}

The effects of the test compounds on the Jurkat cell transcriptome were assessed upon $6 \mathrm{~h}$ of exposure to subcytotoxic concentrations (Table 1). Subcytotoxic concentrations were defined as more than $80 \%$ viability (CV80) and less than $20 \%$ induction of cytotoxicity after $24 \mathrm{~h}$ of exposure. In order to determine these subcytotoxic exposure concentrations, dose-cell viability (WST-1, Roche, and ATPLite, Perkin Elmer) response curves were generated for the test compounds. In addition, the subcytotoxic exposure conditions were verified by employing an AK leakage assay (Toxilight, Lonza; see Supplementary table 2). The cytotoxicity of the 31 compounds ranged from 60 to $120 \%$ of the vehicle control. The S9 mixture, used for bioactivation of BaP, OTA, and CP, was not cytotoxic at 200 $\mu \mathrm{g} / \mathrm{ml}$, which was the final concentration to which the Jurkat cells were exposed (data not shown).

\section{Identification of the Mechanisms of Direct Immunotoxicity by Transcriptome Analyses}

We identified 1445 genes that were modulated by at least one of the immunotoxic chemicals after considering a fold change cutoff of $>2 \log 0.7$ or $<2 \log -0.7$ (numerical $>1.62$ up or down) in at least three of the four biological replicates compared with the average of the vehicle control arrays. We subjected these 1445 genes to unsupervised hierarchical clustering analysis (for a heatmap see Supplementary fig. 2). 


\begin{tabular}{|c|c|c|c|c|c|c|c|c|}
\hline 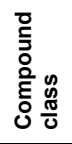 & $\begin{array}{l}\text { } \\
\stackrel{E}{E}\end{array}$ & $\begin{array}{l}\text { } \\
\text { 으 }\end{array}$ & $\begin{array}{l}\text { ○ } \\
\text { E }\end{array}$ & $\begin{array}{l}\text { ㅇ } \\
\text { ㅌ }\end{array}$ & $\begin{array}{l}\text { ○ } \\
\text { ㅇ }\end{array}$ & $\begin{array}{l}\text { 읍 } \\
\text { E }\end{array}$ & $\begin{array}{l}\text { } \\
\text { 으 }\end{array}$ & $\begin{array}{l}\text { Oે } \\
\text { E }\end{array}$ \\
\hline 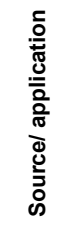 & 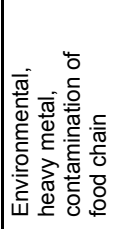 & 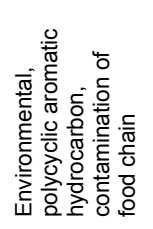 & 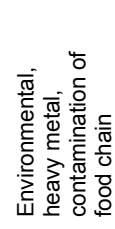 & 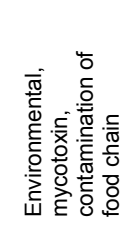 & 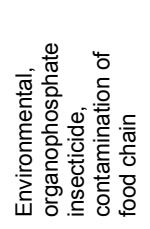 & 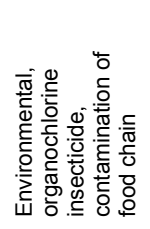 & 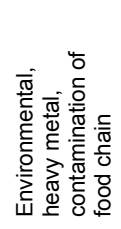 & 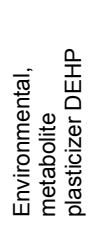 \\
\hline 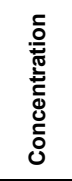 & $\sum_{m}$ & 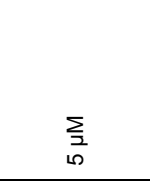 & $\begin{array}{l}\sum_{3} \\
\infty\end{array}$ & 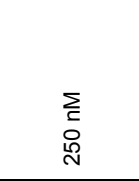 & $\begin{array}{l}\sum_{3} \\
8 \\
\text { ㅇ }\end{array}$ & $\begin{array}{l}\sum_{3} \\
\text { - } \\
\end{array}$ & $\underset{-}{\sum_{2}}$ & $\begin{array}{l}\sum_{3} \\
\text { ᄋे }\end{array}$ \\
\hline 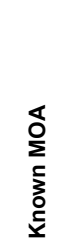 & 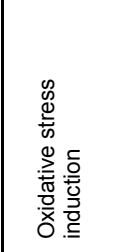 & 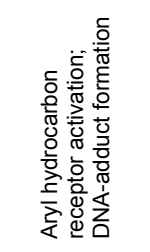 & 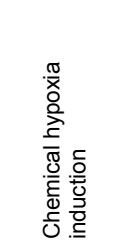 & 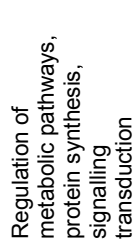 & 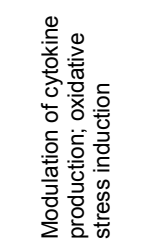 & 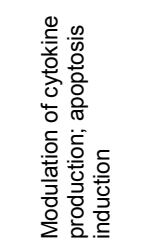 & 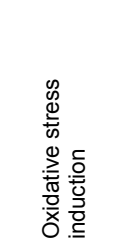 & 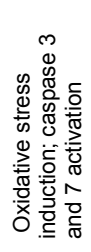 \\
\hline ర্ & 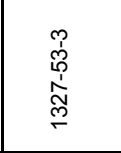 & $\begin{array}{l}\infty \\
\grave{d} \\
\text { ஸे } \\
\text { ம }\end{array}$ & 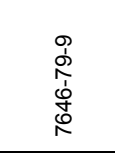 & $\begin{array}{l}\text { o } \\
\dot{1} \\
\frac{1}{1} \\
\frac{1}{\infty} \\
\dot{+} \\
\dot{1}\end{array}$ & 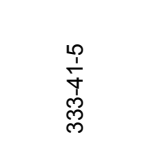 & 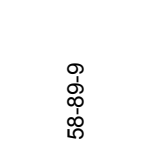 & 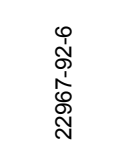 & 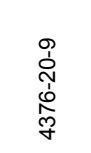 \\
\hline 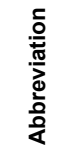 & 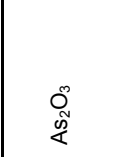 & 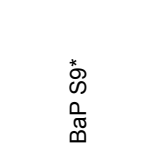 & $\begin{array}{l}\text { ரू } \\
\text { Oे }\end{array}$ & zo & Z & $\underline{\Xi}$ & $\begin{array}{l}\frac{\text { OT }}{1} \\
\frac{1}{2}\end{array}$ & $\begin{array}{l}\stackrel{0}{\mathrm{I}} \\
\stackrel{\mathrm{W}}{\Sigma}\end{array}$ \\
\hline 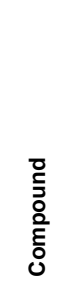 & 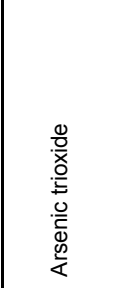 & 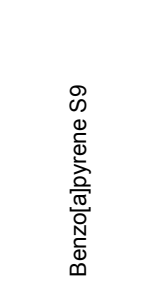 & 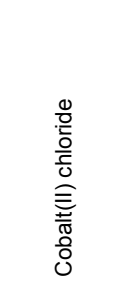 & 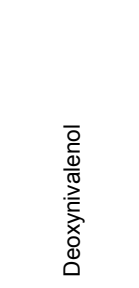 & 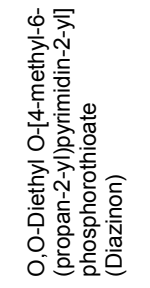 & 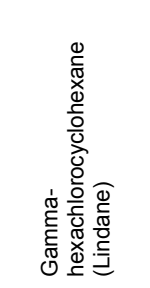 & 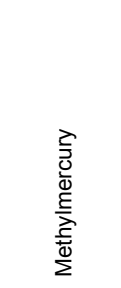 & 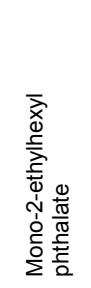 \\
\hline
\end{tabular}




\begin{tabular}{|c|c|c|c|c|c|c|c|c|c|c|c|c|c|}
\hline 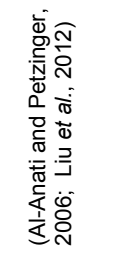 & 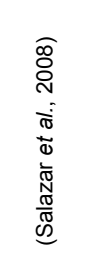 & 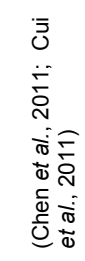 & 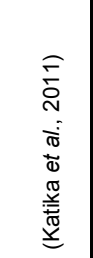 & 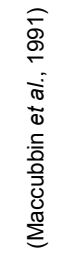 & 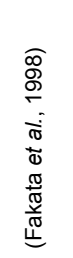 & 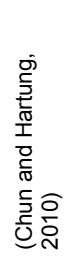 & 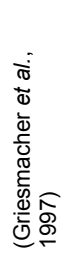 & 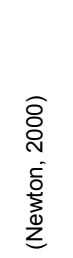 & 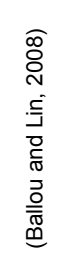 & & 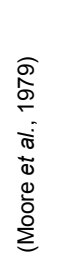 & & 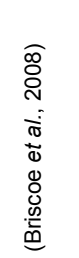 \\
\hline$\overleftrightarrow{5}$ & & $\begin{array}{l}\text { O } \\
\text { 商 }\end{array}$ & $\begin{array}{l}\text { O } \\
\stackrel{\circ}{\Delta}\end{array}$ & 0 & & & & & & & & & \\
\hline 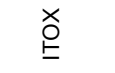 & $\begin{array}{l}\text { Ò } \\
\text { E }\end{array}$ & $\begin{array}{l}\stackrel{x}{\circ} \\
\underline{E}\end{array}$ & $\begin{array}{l}\text { } \\
\stackrel{E}{E}\end{array}$ & $\underline{\text { O) }}$ & 只 & 足 & 只 & 只 & 只 & $\begin{array}{l}\text { zo } \\
\text { z }\end{array}$ & zo & zo & $\begin{array}{l}z \\
\text { z }\end{array}$ \\
\hline 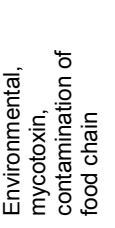 & 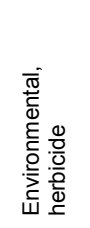 & 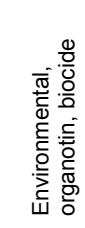 & 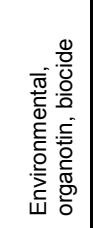 & 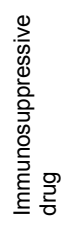 & 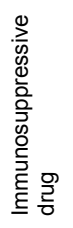 & 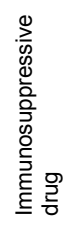 & 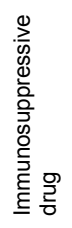 & 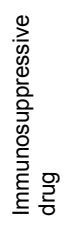 & 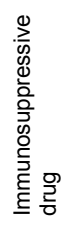 & 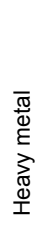 & 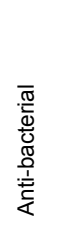 & 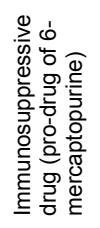 & 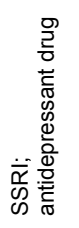 \\
\hline $\begin{array}{l}\sum_{3} \\
\text { O }\end{array}$ & $\begin{array}{l}\sum_{3} \\
\stackrel{8}{0}\end{array}$ & $\begin{array}{l}\sum_{0} \\
8 \\
\stackrel{0}{0}\end{array}$ & $\begin{array}{l}\sum_{c} \\
8 \\
0\end{array}$ & $\sum_{\xi}$ & 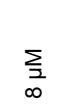 & $\underset{\substack{2 \\
\forall}}{ }$ & $\begin{array}{l}\sum_{3} \\
ㅇ\end{array}$ & $\begin{array}{l}\sum_{3} \\
8 \\
\end{array}$ & $\begin{array}{l}\sum_{c} \\
\stackrel{N}{N}\end{array}$ & 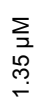 & $\begin{array}{l}\sum_{2} \\
\stackrel{0}{0} \\
\infty\end{array}$ & \begin{tabular}{l}
$\sum_{\leq}$ \\
8 \\
\hdashline
\end{tabular} & $\begin{array}{l}\sum_{3} \\
\text { Oे }\end{array}$ \\
\hline 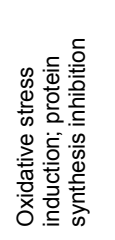 & 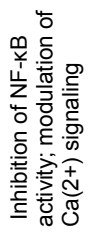 & 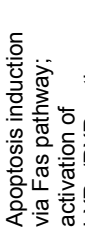 & 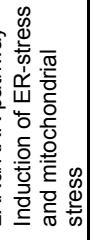 & 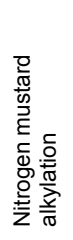 & 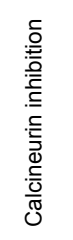 & 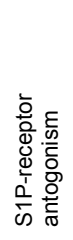 & 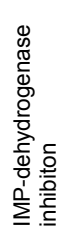 & 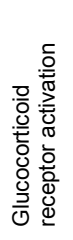 & 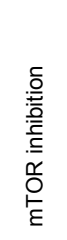 & & 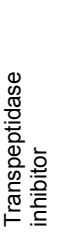 & & $\begin{array}{l}\overline{\bar{\alpha}} \\
\text { ஸे }\end{array}$ \\
\hline 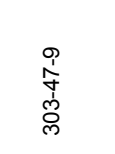 & 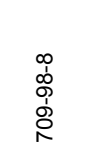 & 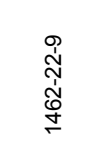 & 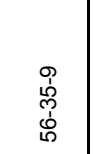 & $\begin{array}{l}0 \\
\infty \\
\\
\stackrel{1}{0}\end{array}$ & 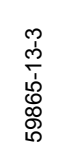 & 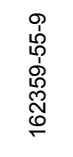 & 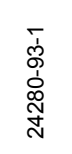 & $\begin{array}{l}\text { o } \\
\text { ஸे } \\
\text { 定 }\end{array}$ & 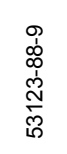 & $\begin{array}{l}\infty \\
0 \\
1 \\
\infty \\
1 \\
\frac{1}{0} \\
\frac{1}{1}\end{array}$ & 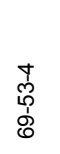 & $\begin{array}{l}0 \\
\dot{1} \\
0 \\
\dot{0} \\
\dot{j} \\
\dot{\gamma}\end{array}$ & 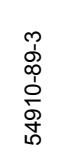 \\
\hline $\begin{array}{l}\text { *o } \\
\text { on } \\
5 \\
5\end{array}$ & $\begin{array}{l}0 \\
0 \\
\frac{x}{\alpha}\end{array}$ & 品 & 음 & $\begin{array}{l}\text { * } \\
\text { 心 } \\
0 \\
0\end{array}$ & 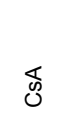 & $\underset{⿱ 亠 䒑}{\stackrel{N}{*}}$ & $\frac{\nwarrow}{\Sigma}$ & $\frac{Q}{\alpha}$ & $\frac{\mathbb{1}}{\underline{\alpha}}$ & 号 & $\sum_{<}^{0}$ & $\underset{\mathbb{Z}}{\mathbb{X}}$ & $\underset{\Downarrow}{\longrightarrow}$ \\
\hline 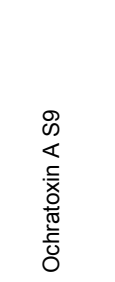 & 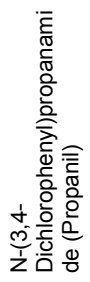 & 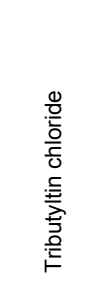 & 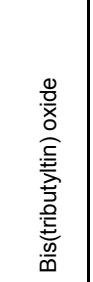 & 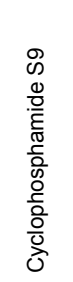 & 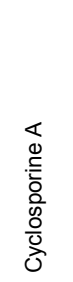 & 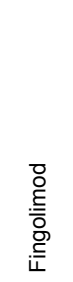 & 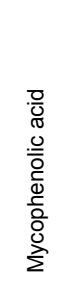 & 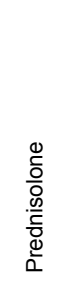 & 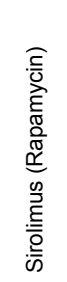 & 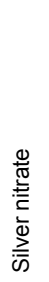 & 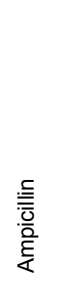 & 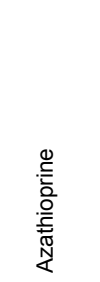 & 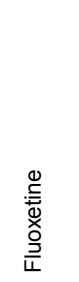 \\
\hline
\end{tabular}




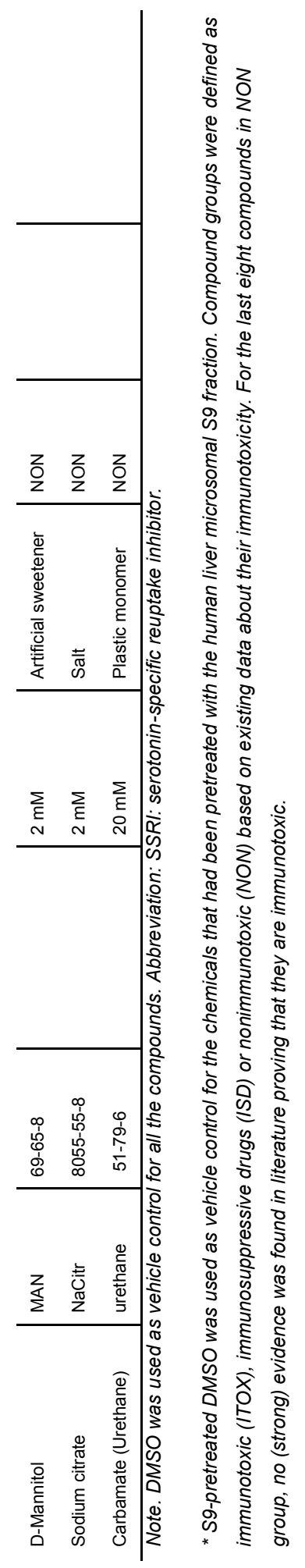




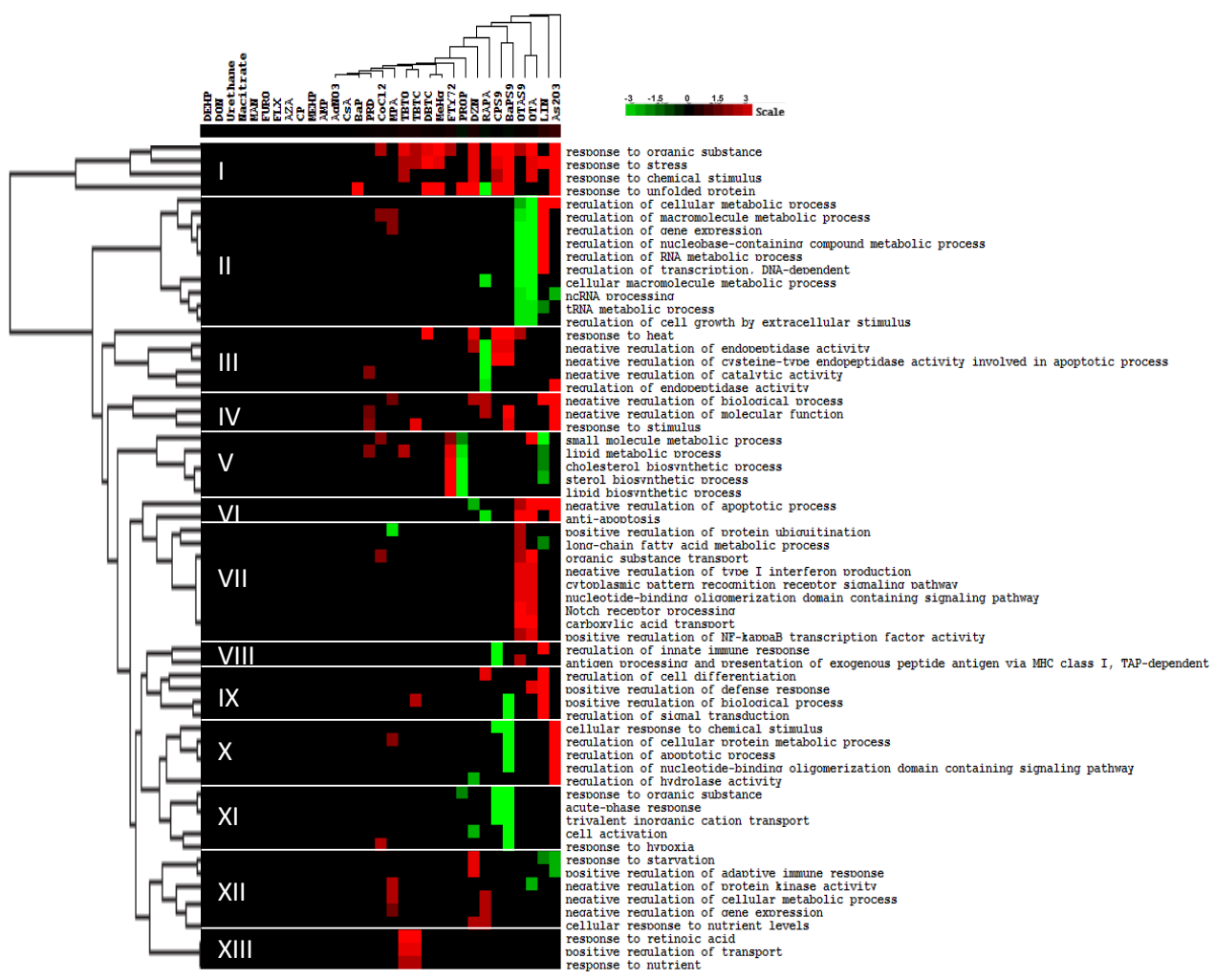

FIG. 1.. Unsupervised hierarchical clustering of GO processes. Heat map visualizes the up- or downregulation of GO processes that were affected by at least two compounds in Jurkat cells upon 6h exposure to subcytotoxic levels of 31 different immunotoxic and nonimmunotoxic chemicals and drugs. Green represents downregulation, black no regulation, and red upregulation. The colour intensity related to the $Z$ value is indicated by the bar ( $Z$ value $>2.72$ is equal to $p$ value $<0.0001$ ). For the abbreviations of all the compounds, see Table 1.

Then, for each of the test compounds, individual GO enrichment analyses were performed on these 1445 genes using Metacore. These analyses led to the identification of $62 \mathrm{GO}$ processes that were enriched by at least two immunomodulating compounds ( $p$ value $<0.01$ ). Thereafter, hierarchical clustering was performed on the $Z$ scores of these enriched GO processes, which resulted in the identification of 13 different clusters of GO processes (Fig. 1). Table 2 summarizes these coclustered GO processes and the compounds involved in each cluster, as well as the individual genes responsible for these enriched processes.

\section{Metacore Analysis- Common Putative Mechanisms of Direct Immunotoxicity}

Two main GO processes were identified as being most commonly activated by the immunomodulating compounds, cellular stress responses (cluster I) and anti-apoptosis (cluster VI) (Fig. 1 and Table 2). 
These clusters were jointly activated by the heavy metal $\mathrm{As}_{2} \mathrm{O}_{3}$, the mycotoxin OTA (+ $\mathrm{S} 9$ and - S9), and the organochlorine insecticide lindane, indicating that these immunotoxicants activate both cellular stress responses and cytoprotective antiapoptotic processes. In contrast, the heavy metal $\mathrm{MeHg}$, the organophosphate insecticide diazinon, the organotin compounds (dibutyltin chloride [DBTC], tributyltin chloride [TBTC], TBTO), the alkylating agent CP (+ S9), and the poly acyclic hydrocarbon BaP (+ S9 and - S9) specifically activated cellular stress responses (cluster I) without activating anti-apoptotic responses (cluster VI).

Various other GO processes were regulated by three or four immunomodulating compounds and were found among clusters II, III, IV, V, VIII, XI, and XII (Fig. 1 and Table 2). Cluster II includes the regulation of cell proliferation, gene transcription, and protein translation. These processes were mainly activated by lindane and inhibited by OTA (+ S9 and - S9). Heat shock responses (within cluster III) were activated by BaP (+ S9), CP (+ S9), diazinon, DBTC, and OTA (+ S9). Cluster IV includes negative regulation of biological processes and molecular functions. These processes were mainly found to be activated by $\mathrm{As}_{2} \mathrm{O}_{3}, \mathrm{BaP}$ (+ S9), prednisolone, and rapamycin. Cholesterol and lipid metabolism (cluster V) were activated by the S1PR antagonist FTY720 and inhibited by lindane and propanil. Immunomodulation (cluster VIII) was activated by lindane and OTA (+ S9) and inhibited by CP (+ S9). Responses to organic substances (within cluster XI) were inhibited by diazinon, BaP (+ S9) and CP (+ S9). This process was also affected in cluster I, but by another subset of genes (Table 2). Starvation responses (cluster XII) were activated by diazinon and inhibited by $\mathrm{As}_{2} \mathrm{O}_{3}$ and lindane.

\section{Metacore Analysis- Other Mechanisms of Direct Immunotoxicity}

We also identified $\mathrm{GO}$ processes that were specifically affected by one or two of the immunomodulating compounds. $\mathrm{CoCl}_{2}$ specifically induced the expression levels of genes involved in hypoxia (Fig. 1, cluster XI). OTA (+ S9 and - S9) altered the expression levels of genes involved in cytoprotection, lipid metabolism, and type I interferon production (cluster VII). In addition, the genes of this cluster are involved in Notch and NF-KB signaling. Lindane and BaP (+ S9) altered the expression levels of genes involved in signal transduction related to immunity and antiapoptosis (cluster IX). $\mathrm{As}_{2} \mathrm{O}_{3}$ and $\mathrm{BaP}(+\mathrm{S} 9)$ altered the expression levels of genes involved in cellular stress responses related with metabolism and apoptosis (cluster X). Tributyltin compounds (TBTC and TBTO) induced the expression levels of genes involved in response to retinoic acid, and metabolism and transport of lipids (cluster XIII). These effects were highly specific for tributyltins because other compounds, including DBTC had no effects on the expression levels of these genes.

\section{Gene Set Enrichment Analysis (GSEA)}

In order to confirm the results of Metacore, we applied GSEA on the full transcriptome data of each individual compound using gene set collections from the GO consortium. The gene sets that were enriched by at least two immunomodulating compounds ( $p$ value $<0.01)$ are shown in Supplementary fig. 3 . 
In general, the results from GSEA analyses confirmed most of the biological processes and molecular functions we identified with Metacore. Cell cycle control and regulation of transcription and translation were identified as the most commonly affected biological processes by immunomodulating compounds (Supplementary fig. 3A). Almost half of the compounds (15/31) affected at least one gene set that is related with cell cycle, transcription, or translation. This number of affected compounds is much higher than the results from Metacore analyses, which is expected as the statistical model of GSEA is more sensitive than Metacore. Furthermore, cellular stress responses (ER stress affected by S9-treated OTA and CP, DON, CsA, and DBTC; and hypoxia affected by lindane and $\mathrm{CoCl}_{2}$ ) and transport of lipid (affected by TBTO and TBTC) and amino acids (affected by lindane, $\mathrm{As}_{2} \mathrm{O}_{3}$, S9-treated $\mathrm{CP}$ and $\mathrm{BaP}$ ) were also detected by GSEA (Supplementary fig. $3 \mathrm{~A}$ and $3 C)$. In addition, GSEA analyses using the gene sets from the cellular component database of the GO consortium also revealed that a number of compounds, lindane, TBTO, fluoxetine, prednisolone, diazinon, rapamycin, $\mathrm{As}_{2} \mathrm{O}_{3}$, and propanil, downregulated the gene sets related with ribonucleoprotein complex, nucleolus, and mitochondria (Supplementary fig. 3B). 


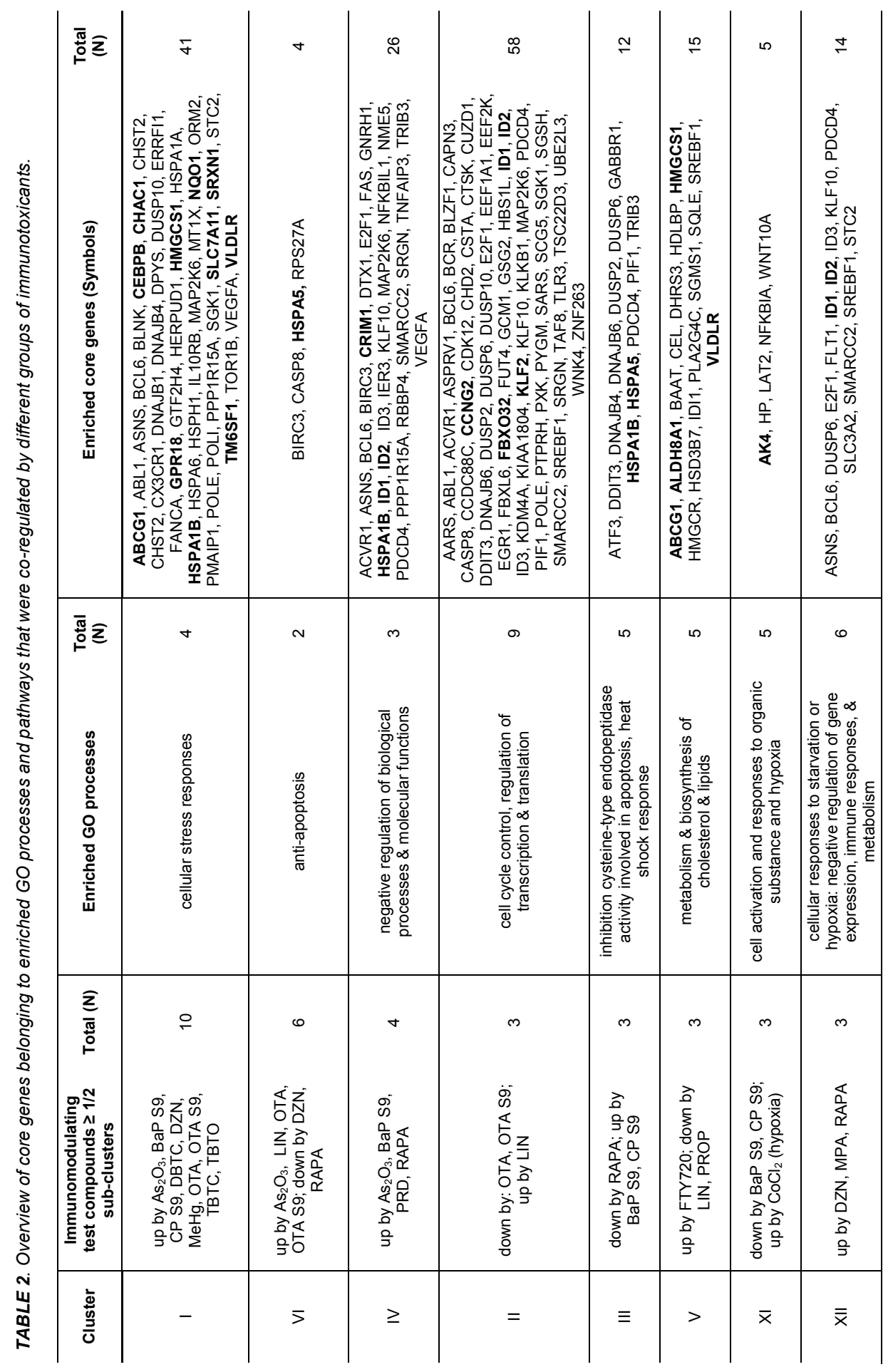




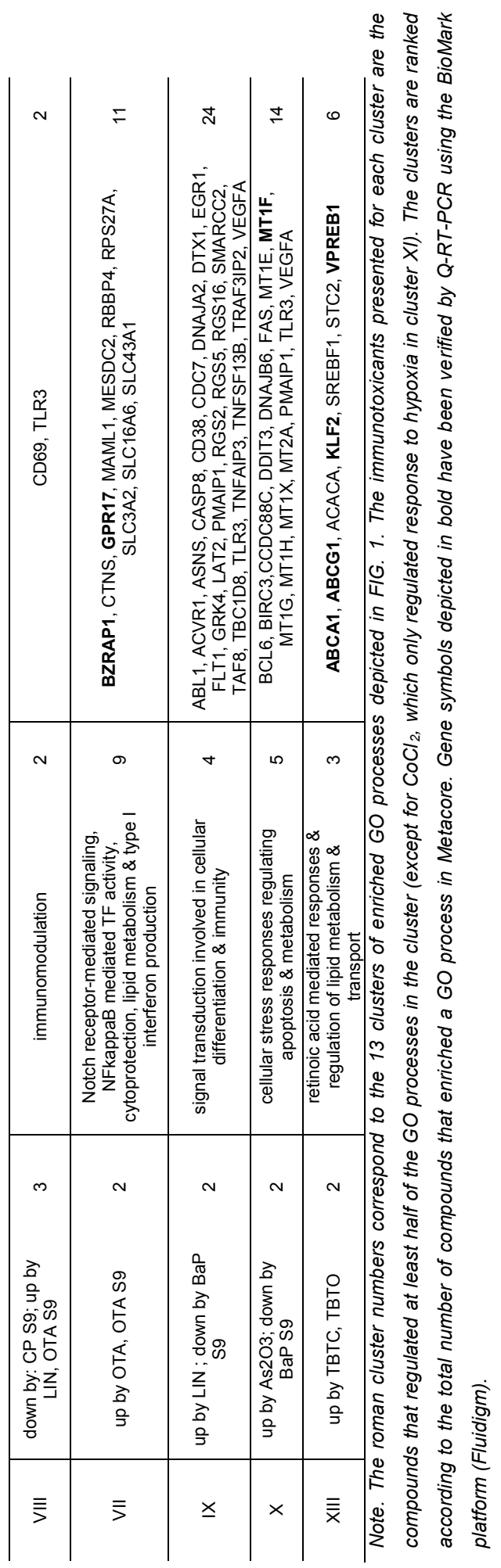




\section{Data Comparison Using the Outcome of Other Toxicogenomics Studies}

In order to determine the degree of overlap between our data and the data from other toxicogenomics studies, we selected the genes which were up- or downregulated, after considering a fold change cutoff of $>2 \log 0.7$ or $<2 \log -0.7$ (numerical> 1.62 up or down) in at least three of the four biological replicates, for three model compounds, $\mathrm{As}_{2} \mathrm{O}_{3}, \mathrm{TBTO}$, and CsA. The data of $\mathrm{As}_{2} \mathrm{O}_{3}$ were compared with the data of $\mathrm{As}_{2} \mathrm{O}_{3}$-treated human myeloma cells (Matulis et al., 2009) and human liver cells HepG2 (Kawata et al., 2007) (Supplementary fig. 4). The data of TBTO were compared with the data of TBTO-treated mouse thymocytes (van Kol et al., 2012) and HepG2 cells (Magkoufopoulou et al., 2012) (Supplementary fig. 5). The data of CsA were compared with the data of peripheral blood mononuclear cells (PBMCs) that were isolated from CsA-treated patients (Brouard et al., 2010), and also with the data of CsA-treated HepG2 cells (Magkoufopoulou et al., 2012) (Supplementary fig. 6).

For $\mathrm{As}_{2} \mathrm{O}_{3}$, part of the genes that were up- or downregulated in Jurkat cells were also shown to be affected to the same direction in myeloma cells and HepG2 cells (Supplementary fig. 4). However, the degree of overlap is clearly higher in myeloma cells (Supplementary fig. 4A) than in HepG2 cells (Supplementary fig. 4B).

For TBTO, most of the genes that were upregulated in Jurkat cells were also shown to be upregulated after 3 and $6 \mathrm{~h}$ of exposure to $0.5 \mu \mathrm{M}$ of TBTO in mouse thymocytes (Supplementary fig. 5A). We have also identified some overlap in the upregulated genes between our data set and that of TBTO- treated HepG2 cells (Supplementary fig. 5B). Only four genes were downregulated by TBTO in Jurkat cells after considering the fold change cutoff ( $>2 \log 0.7$ or $<2 \log -0.7$ ), and none of them can be found in the data of TBTO-treated mouse thymocytes or HepG2 cells.

For CsA, most of the genes that were upregulated in Jurkat cells were also shown to be upregulated in 13 out of $14 \mathrm{CsA}$-treated patients (Supplementary fig. 6A, genes upregulated in Jurkat). Only four genes were downregulated by CsA in Jurkat cells after considering the fold change cutoff ( $>2 \log 0.7$ or $<2 \log -0.7$ ), and the expression levels of these found genes were quite random among the patients (Supplementary fig. 6A, genes downregulated in Jurkat cells). Not much similarity can be found between our data and the data of CsA-treated HepG2 cells (Supplementary fig. 6B).

\section{Verification of the Microarray Gene Expression Patterns by Quantitative Real-Time PCR}

We proceeded with verifying the microarray mRNA expression patterns and relevant GO processes that were associated with exposure to direct immunotoxicants. For this purpose, we employed quantitative real-time PCR (Q-RT-PCR) on three out of four independent experiments that were also used for the microarray experiments. Out of the 1445 genes that were transcriptionally regulated by one or more compounds, we selected 28 signature genes by using the following criteria: we first identified 93 genes, consisting of 80 genes that were modulated by at least three compounds, and 13 genes that were specifically regulated by one single compound or compound class. Then we selected 
28 genes out of these 93 genes, based on the largest up- or downregulation in response to the immunotoxicants.

As shown in Tables 2 and 3, these 28 genes can be linked to $G O$ processes that are potentially relevant for immunotoxicity. From these 28 genes, 10 genes are from cluster I, being CHAC1, GPR18, HMGCS1, HSPA1B, HSPA5, NQO1, SLC7A11, SRXN1, TM6SF1, and VLDLR, and are associated with cellular stress responses (cluster I, Table 2). CHAC1, HSPA1B, and HSPA5 are well- known markers for ER stress and the unfolded protein response (UPR). NQO1 and SRXN1 are specifically related with response to oxidative stress. HSPA1B and HSPA5 are also part of cluster III and are involved in heat shock responses. HSPA5 is also one of the cluster $\mathrm{VI}$ genes and is involved in antiapoptosis. The cluster II genes CCNG2, ID1, and ID2 are involved in cell cycle control and regulation of gene transcription and protein translation.

HMGCS1, VLDLR, and ABCG1 are from cluster $V$ and associated with metabolism, biosynthesis, and transport of cholesterol and lipids. ABCG1, together with ABCA1, KLF2, and VPREB1, is part of cluster XIII genes and is associated with response to retinoic acid. The cluster XI gene AK4 and cluster VII gene GPR17 are specifically related with response to hypoxia and G protein-coupled cell signalling, respectively.

For 27 of the 28 genes, the Q-RT-PCR based expression patterns generally correlated well with the microarray data (Pearson correlation, $R \geq 0.69$ ) (Table 3 ). The only exception here was NANOS1 $(R=0.14)$ that was excluded from further comparisons. Furthermore, for three genes and two compounds, the mRNA expression patterns showed opposite effects between the Q-RT-PCR and the microarray data, ID2 for lindane, and GPR18 and HSPA5 for OTA. For all other test compounds, QRT-PCR gave similar results for GPR18, HSPA5, and ID2 as the microarray (Pearson correlation, $R \geq 0.69$ ). Examples of the comparison between the results from microarray and the Q-RT-PCR are given in Figure 2. In general, the effects found in Q-RT-PCR analysis were larger than those shown by microarray analysis. This suggests that Q-RT-PCR was a more sensitive method to detect chemically induced gene expression changes than microarray mRNA expression profiling. 
TABLE 3. Major GO processes and molecular functions in which the 28 genes are involved, Pearson correlation results between the microarray and Q-RT-PCR data, and the putative molecular targets of the 28 genes.

\begin{tabular}{|c|c|c|c|c|c|c|}
\hline $\begin{array}{c}\text { Gene } \\
\text { symbol }\end{array}$ & $\begin{array}{c}\text { mRNA } \\
\text { accession }\end{array}$ & $\begin{array}{l}\text { Affymetrix } \\
\text { ID }\end{array}$ & Taqman assay & $\begin{array}{l}\text { Pearson } \\
\text { correlation } \\
\mathbf{R}\end{array}$ & $\begin{array}{l}\text { GO biological process I } \\
\text { molecular function (Gene } \\
\text { Ontology, Genecards) }\end{array}$ & $\begin{array}{l}\text { Putative } \\
\text { molecular } \\
\text { targets }\end{array}$ \\
\hline CCNG2 & NM_004354 & 901_at & Hs00171119_m1 & 0.86 & regulation of cell cycle & $\begin{array}{l}\text { FOXO (Chen } \\
\text { et al., 2006) }\end{array}$ \\
\hline HMGCS1 & NM_001098272 & 3157_at & Hs00940429_m1 & 0.84 & $\begin{array}{c}\text { cholesterol biosynthesis } \\
\text { SREBP }\end{array}$ & $\begin{array}{c}\text { SREBP } \\
\text { (Bensinger et } \\
\text { al., 2008) } \\
\end{array}$ \\
\hline ALDH8A1 & NM_001193480 & 64577_at & Hs00988965_m1 & 0.83 & $\begin{array}{l}\text { metabolism and biosynthesis } \\
\text { of retinoic acid }\end{array}$ & \\
\hline BZRAP1 & NM_004758 & 9256_at & Hs00270490_m1 & 0.94 & $\begin{array}{l}\text { benzodiazepine receptor } \\
\text { binding }\end{array}$ & \\
\hline NANOS1 & NM_199461 & 340719_at & H00996068_s1 & 0.14 & $\begin{array}{l}\text { inhibition of translation, zinc } \\
\text { ion binding }\end{array}$ & \\
\hline $\mathrm{FBXO} 32$ & NM_001242463 & 114907_at & Hs01041408_m1 & 0.92 & protein ubiquitination & $\begin{array}{l}\text { TGF beta/ } \\
\text { SMAD4 } \\
\text { (Chou et al., } \\
\text { 2010; Tan et } \\
\text { al., 2007) } \\
\end{array}$ \\
\hline ARRDC3 & NM_020801 & 57561_at & Hs00385845_m1 & 0.84 & protein binding & \\
\hline CRIM1 & NM_016441 & 51232_at & Hs00212750_m1 & 0.82 & $\begin{array}{l}\text { insulin-like growth factor } \\
\text { receptor activity, serine-type } \\
\text { endopeptidase inhibitor } \\
\text { activity, regulation of cell } \\
\text { growth }\end{array}$ & \\
\hline GPR17 & NM_001161415 & 2840_at & Hs00171137_m1 & 0.92 & $\begin{array}{l}\text { G-protein coupled receptor } \\
\text { signaling, Dual specificity } \\
\text { receptor for uracil nucleotides } \\
\text { and cysteinyl leukotrienes } \\
\text { (Genecards) }\end{array}$ & \\
\hline GPR18 & NM_001098200 & 2841_at & Hs00245542_m1 & 0.73 & $\begin{array}{l}\text { G-protein coupled receptor } \\
\text { signaling, N-arachidonyl } \\
\text { glycine receptor (Genecards) }\end{array}$ & \\
\hline KLHL24 & NM_017644 & 54800_at & Hs00214210_m1 & 0.81 & protein binding & \\
\hline ABCA1 & NM_005502 & 19_at & Hs01059118_m1 & 0.92 & $\begin{array}{l}\text { response to retinoic acid, } \\
\text { ATP-dependent } \\
\text { transmembrane transport of } \\
\text { sterols }\end{array}$ & \multirow{4}{*}{$\begin{array}{l}\text { LXR, RAR, } \\
\text { RXR } \\
\text { heterodimers } \\
\text { (Berry et al., } \\
\text { 2012; Costet } \\
\text { et al., 2003; } \\
\text { Levin et al., } \\
\text { 2005) } \\
\text { (Tontonoz } \\
\text { and } \\
\text { Mangelsdorf, } \\
\text { 2003) }\end{array}$} \\
\hline ABCG1 & NM_004915 & 9619_at & Hs00245154_m1 & 0.87 & $\begin{array}{c}\text { response to retinoic acid, } \\
\text { ATP-dependent } \\
\text { transmembrane transport of } \\
\text { purine nucleotides, L- } \\
\text { tryptophan, and sterols }\end{array}$ & \\
\hline KLF2 & NM_016270 & 10365_at & Hs00360439_g1 & 0.91 & $\begin{array}{c}\text { response to stress, } \\
\text { transcription factor activity, } \\
\text { regulates T-cell } \\
\text { trafficking by promoting } \\
\text { expression of the lipid- } \\
\text { binding receptor S1P1 and } \\
\text { the selectin CD62L } \\
\text { (Weinreich et al., 2009) }\end{array}$ & \\
\hline VPREB1 & NM_007128 & 7441_at & Hs00356766_g1 & 0.72 & $\begin{array}{l}\text { immune response, antigen } \\
\text { binding }\end{array}$ & \\
\hline MT1F & NM_005949 & 4494_at & Hs01582977_gH & 0.95 & $\begin{array}{l}\text { response to metal ion and } \\
\text { oxidative stress }\end{array}$ & $\begin{array}{l}\text { MTF1 }(\mathrm{He} \\
\text { and } \mathrm{Ma} \text {, } \\
2009)\end{array}$ \\
\hline
\end{tabular}




\begin{tabular}{|c|c|c|c|c|c|c|}
\hline CHAC1 & NM_001142776 & 79094_at & Hs00225520_m1 & 0.84 & $\begin{array}{l}\text { possibly pro-apoptotic } \\
\text { component of unfolded } \\
\text { protein response by } \\
\text { mediating the ATF4-ATF3- } \\
\text { DDIT3/CHOP cascade } \\
\text { (Genecards), negative } \\
\text { regulaton Notch signaling } \\
\text { (Mungrue et al., 2009) }\end{array}$ & $\begin{array}{l}\text { ATF4-ATF3- } \\
\text { DDIT3/CHOP } \\
\text { cascade } \\
\text { (Mungrue et } \\
\text { al., 2009) }\end{array}$ \\
\hline HSPA1B & NM_005346 & 3304_at & Hs01040501-sH & 0.81 & $\begin{array}{l}\text { Response to unfolded } \\
\text { protein, anti-apoptosis HSF1 }\end{array}$ & $\begin{array}{l}\text { HSF1 (Song } \\
\text { et al., 2010) }\end{array}$ \\
\hline HSPA5 & NM_005347 & 3309_at & Hs00946084_g1 & 0.69 & $\begin{array}{c}\text { response to unfolded protein, } \\
\text { starvation response, involved } \\
\text { in the folding and assembly } \\
\text { of proteins in the ER } \\
\text { (Genecards) ATF6 and XBP- } \\
1\end{array}$ & $\begin{array}{l}\text { ATF6 and } \\
\text { XBP-1 } \\
\text { (Schröder } \\
\text { and } \\
\text { Kaufman, } \\
\text { 2005) } \\
\end{array}$ \\
\hline NQO1 & NM_000903 & 1728_at & Hs01045995_m1 & 0.91 & $\begin{array}{c}\text { response to toxin, } \mathrm{NAD}(\mathrm{P}) \mathrm{H} \\
\text { dehydrogenase (quinone) } \\
\text { activity }\end{array}$ & \multirow{2}{*}{$\begin{array}{l}\text { KEAP1/ } \\
\text { NRF2 } \\
\text { (Schäfer et } \\
\text { al., 2010) }\end{array}$} \\
\hline SRXN1 & NM_080725 & 140809_at & Hs00607800_m1 & 0.69 & $\begin{array}{l}\text { response to oxidative stress, } \\
\text { antioxidant activity }\end{array}$ & \\
\hline ID1 & NM_002165 & 3397_at & Hs00357821_g1 & 0.79 & $\begin{array}{c}\text { regulation of transcription } \\
\text { from RNA polymerase II } \\
\text { promoter, regulation of } \\
\text { apoptosis }\end{array}$ & \multirow{2}{*}{$\begin{array}{c}\text { BMP } \\
\text { signaling } \\
\text { (Hollnagel et } \\
\text { al., 1999) } \\
\text { Wnt signaling } \\
\text { (Katoh and } \\
\text { Katoh, 2009) } \\
\end{array}$} \\
\hline ID2 & NM_002166 & 3398_at & Hs00747379_m1 & 0.73 & $\begin{array}{l}\text { Immune cell differentiation, } \\
\text { inhibition of gene } \\
\text { transcription }\end{array}$ & \\
\hline AK4 & NM_001005353 & 205_at & Hs03405743_g1 & 0.79 & $\begin{array}{l}\text { amino acid biosynthesis, } \\
\text { adenylate kinase activity }\end{array}$ & $\begin{array}{l}\text { HIF1A } \\
\text { (Greijer et } \\
\text { al., 2005) } \\
\end{array}$ \\
\hline SLC7A11 & NM_014331 & 23657_at & Hs00921938_m1 & 0.88 & $\begin{array}{l}\text { response to oxidative stress, } \\
\text { response to toxin, } \\
\text { cystine:glutamate antiporter } \\
\text { activity }\end{array}$ & $\begin{array}{l}\text { elF2a, ATF4 } \\
\text { (Sikalidis et } \\
\text { al., 2011) }\end{array}$ \\
\hline VLDLR & NM_001018056 & 7436_at & Hs01047538_m1 & 0.81 & $\begin{array}{c}\text { ER stress response } \\
\text { (Dombroski et al., 2010), } \\
\text { lipoprotein receptor activity }\end{array}$ & $\begin{array}{c}\text { ATF4 } \\
\text { (Jakobsen et } \\
\text { al., 2008; Jo } \\
\text { et al., 2012) }\end{array}$ \\
\hline CEBPB & NM_005194 & 1051_at & Hs00270923_s1 & 0.81 & $\begin{array}{l}\text { transcriptional activation of } \\
\text { immune and inflammatory } \\
\text { response genes (Genecards) }\end{array}$ & \\
\hline TM6SF1 & NM_001144903 & 53346_at & Hs00224823_m1 & 0.92 & $\begin{array}{c}\text { ER stress response (Taylor } \\
\text { et al., 2011) }\end{array}$ & \\
\hline
\end{tabular}



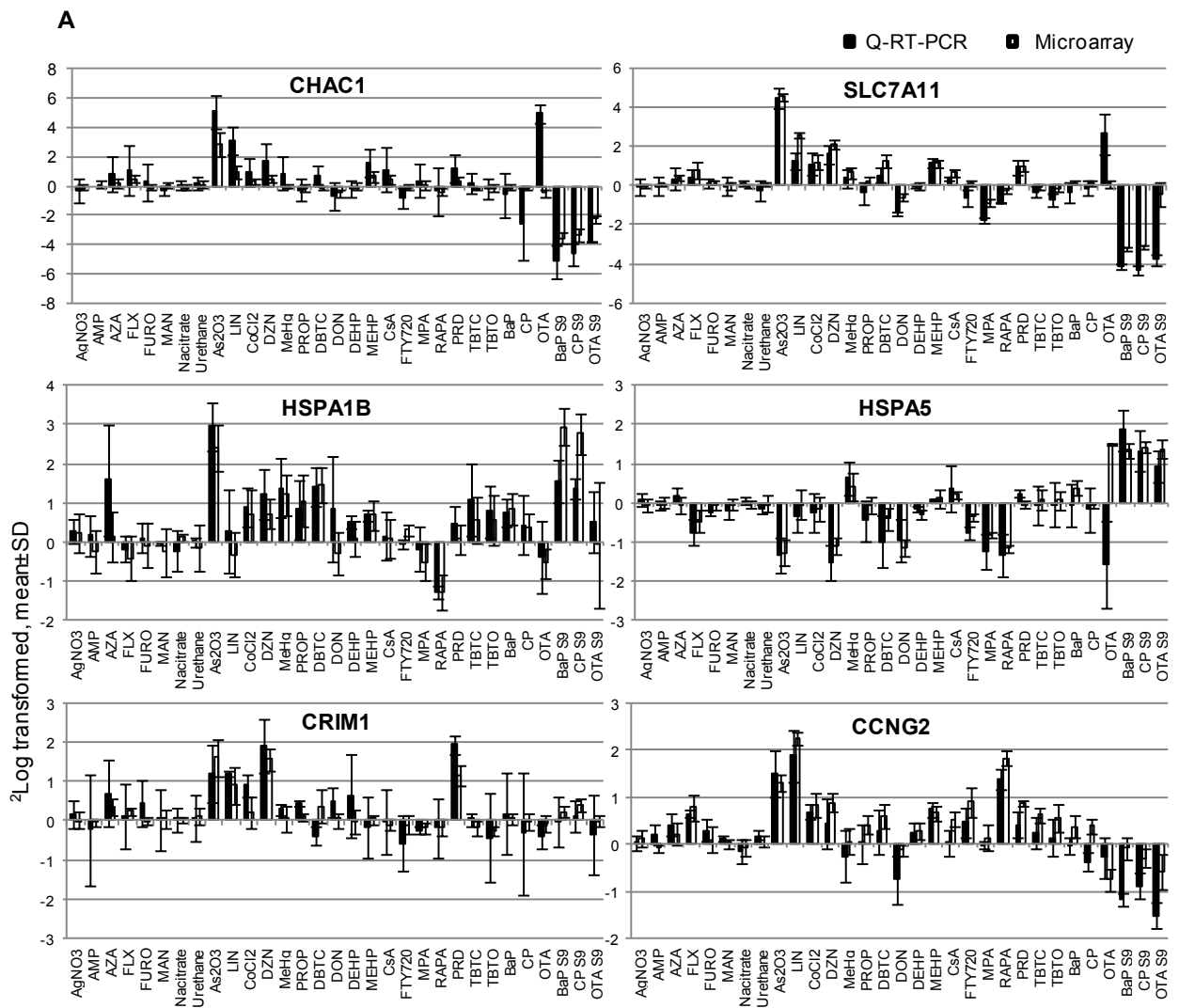

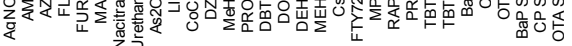
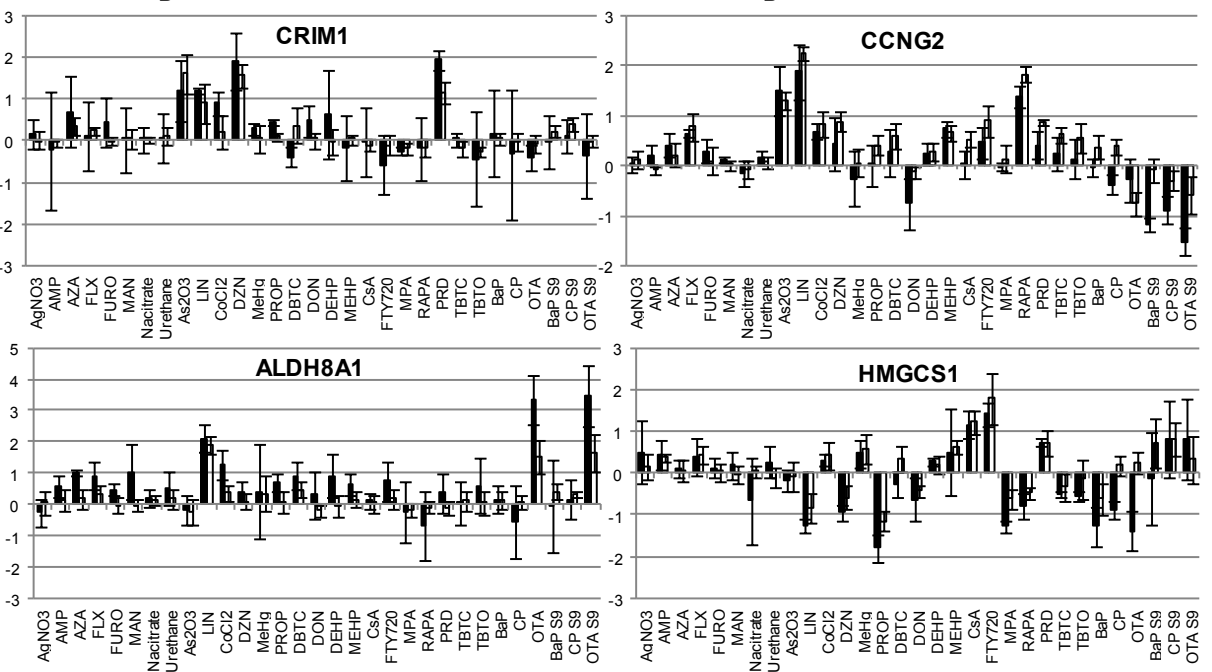

FIG. 2. Verification of the microarray gene expression patterns by Q-RT-PCR. The bar graphs show the comparison between the data of microarray and Q-RT-PCR verification for 16 core genes from different clusters as examples: A) CHAC1, SLC7A11, and HSPA1B from cluster I; HSPA5 from cluster VI; CRIM1 from cluster IV; CCNG2 from cluster II; ALDH8A1 and HMGCS1 from cluster V; B) MTF1 from cluster X; AK4 from cluster XI; ID1 and ID2 from cluster XII; GPR17 from cluster VII; GPR18 from cluster I; ABCA1 and ABCG1 from cluster XIII. Each roman cluster number and its core genes correspond to the content of Table 2. For the abbreviations of all the compounds, see Table 1. 
B
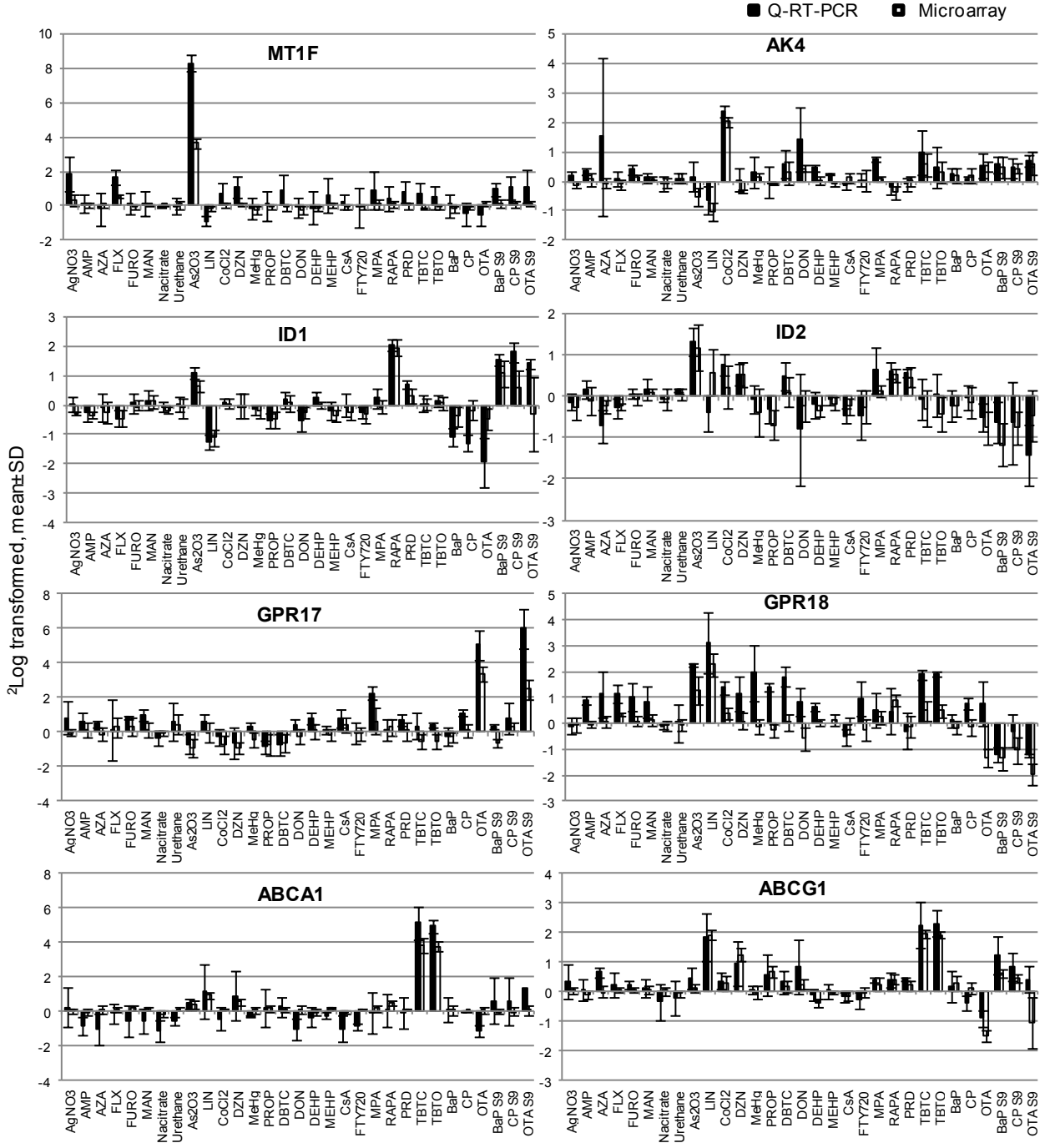

FIG. 2. Continued. 


\section{Discussion}

The present study was aimed at the identification of mechanisms for chemical-induced direct immunotoxicity. To that end, we assessed the in vitro effects of a large variety of immunotoxic and nonimmunotoxic test compounds on the transcriptome of the human T cell line Jurkat.

For three compounds also the effect of biotransformation on gene expression was examined by in vitro treatment of the compounds with human liver S9. BaP, OTA, and CP were chosen because it was shown by others that these compounds need to be bio-activated before exerting their immunomodulatory effects (Carlson et al., 2004; Ekhart et al., 2009; Manderville, 2005). In addition, this issue was addressed by including immunotoxic metabolites of TBTC and bis(2ethylhexyl)phthalate (DEHP), being DBTC and MEHP, respectively. For TBTC, it is known from literature that both the parent compound and its metabolite DBTC are immunotoxic because they can both cause atrophy in rat thymus (Snoeij et al., 1988). For DEHP, it is known to be rapidly hydrolysed into MEHP in vivo (Koch et al., 2006), and MEHP is immunotoxic to immune cells in vitro (RosadoBerrios et al., 2011; Schlezinger et al., 2004).

The biological interpretation of the genes that were up- or downregulated by the exposures enabled the identification of the processes affected by immunotoxicants. Some cellular pathways, processes, and functions were commonly modulated by three or more immunotoxic compounds, whereas other mechanisms were specifically affected by one or two immunotoxicants. The processes most commonly affected by direct immunotoxicants included ER stress, oxidative stress, antiapoptotic responses, negative regulation of biological process and molecular functions, cell cycle control, regulation of transcription and translation, and metabolism of cholesterol and lipids (Fig. 1 and Table 2). The involvement of these mechanisms in the transcriptional responses of Jurkat $T$ cells to direct immunotoxicants was substantiated by Q-RT-PCR analysis.

On the basis of the enriched core genes involved in cellular stress responses (cluster I, Table 2), we could identify two types of cellular stress responses, ER stress/ UPR (CHAC1, HSPA1B, HSPA5, SLC7A11) and oxidative stress (NQO1, SRXN1, SLC7A11). The induction of ER stress can be triggered by continued accumulation of incorrectly folded proteins in the ER (Kaufman et al., 2002), or by compounds that directly affect the function of ER, such as thapsigargin (Treiman et al., 1998). ER stress leads to activation of several transcription factors including ATF3, ATF4, ATF6, and DDIT3 (Galluzzi et al., 2012). Prolonged ER stress can initiate terminal programs in the cells, such as autophagy and apoptosis (Chakrabarti et al., 2011). In T cells, ER stress leads to increased intracellular calcium levels and eventually to T-cell activation (Katika et al., 2011, 2012a). A number of compounds inducing the ER stress response in our study also have been shown to induce this response in other studies. For instance, both $\mathrm{As}_{2} \mathrm{O}_{3}$ and $\mathrm{MeHg}$ have been found to induce $\mathrm{ER}$ stress in myoblasts in vitro (Usuki et al., 2008; Yen et al., 2012). HSPA5 (also upregulated by S9-treated BaP in our experiment, see Supplementary fig. 1), which is a well-known marker gene for ER stress (Galluzzi et al., 2012), has been identified recently as a biomarker for BaP-induced immunotoxicity in Xenopus laevis (Martini et al., 2012). ER stress has also been demonstrated as one of the 
overrepresented pathways for immunotoxicity in a previous toxicogenomics study (Hochstenbach et al., 2012). Our results further supported ER stress as being one of the major mechanisms for immunotoxicity.

The induction of oxidative stress has also been implied in cluster I as two well- known oxidative stress marker genes, NQO1 and SRXN1, are among the enriched core genes of this cluster (cluster I, Table 2). SLC7A11 in this cluster encodes the transporter of cysteine/ glutamate (see the expression pattern of SLC7A11 in fig. 2). This transporter plays an important role in maintaining cellular glutathione level, which is crucial in protecting cells against oxidative stress (Sakakura et al., 2007). Oxidative stress has been shown to be involved in in vivo and in vitro immunotoxicity induced by a wide range of chemicals, such as fungal toxins, heavy metals, and organic substances (Ashry et al., 2010; Hannam et al., 2010; Liu et al., 2012; Mishra et al., 2008). NQO1 and SRXN1 are also known as the downstream target genes of NRF2 pathway (Schäfer et al., 2010). The induction of oxidative stress, mainly through activation of the NRF2 pathway, has also been identified as the key mechanism to distinguish skin sensitizers from irritants (Vandebriel et al., 2010), thus emphasizing the relevance of this pathway for immunotoxicity in a broad sense.

Besides these two types of stress responses, the response to hypoxia was also identified as being a potential relevant process for immunotoxicity (cluster XI, Fig. 1 and Table 2). This response was specially induced by $\mathrm{CoCl}_{2}$, which is a well-known hypoxia-mimetic agent (Stenger et al., 2011) and can activate gene expression in a HIF1a-dependent fashion (Gao et al., 2012). Cobalt chromium has also been shown to induce hypoxia in fibroblasts in vitro (Madathil et al., 2010). The $\mathrm{CoCl}_{2}$ mediated induction of hypoxia could be confirmed by Q-RT-PCR, as AK4, a well-known hypoxia-inducible gene (Greijer et al., 2005), was highly induced by $\mathrm{CoCl}_{2}$ (Fig. 2). Hypoxia increases the production of reactive oxygen species in the mitochondria, which eventually leads to oxidative stress and cell death (Duranteau et al., 1998; Guillemin and Krasnow, 1997; Wang et al., 2000). Some cohort studies suggested that patients with metal-on-metal implants are at a higher risk of developing lymphopenia and metal hypersensitivity because of the cobalt and chromium nanoparticles released from the bearing surface of these devices (Gill et al., 2012). One possible cause for this could be the induction of hypoxia.

Another stress response, response to metals, was found to be highly induced by the heavy metal $\mathrm{As}_{2} \mathrm{O}_{3}$, which is related to the induction of several metallothioneins (MTs) (cluster X, Table 2, also see Supplementary fig. 1). We selected one MT, MT1F, for Q-RT-PCR analysis and could verify the increased expression of this gene as initially observed in the microarray experiment (Fig. 2). MTs can bind to heavy metals, especially to cadmium, and control cellular stresses such as hypoxia and oxidative stress (Otsuka et al., 2007; Wang et al., 2004). MT-null mice are more susceptible to the hematotoxic and immunotoxic effects of cadmium exposure, indicating the involvement of MTs in the detoxification of heavy metals and cytoprotection (Liu et al., 1999). MTs have also been suggested to act as chemoattractants to support the movement of leukocytes to the site of inflammation, which 
further supported their important roles in the immunomodulation against toxicant exposure (Yin et al., 2005).

Microarray data analysis at the level of GO processes also led to the observation that genes involved in the inhibition of apoptosis were commonly activated by direct immunotoxicants, including $\mathrm{As}_{2} \mathrm{O}_{3}$, lindane, and OTA (+ S9 and - S9) (cluster VI, Fig. 1 and Table 2). This observation indicates that transcriptional activation of anti-apoptotic genes is possibly a common cytoprotective mechanism upon exposure to direct immunotoxicants. We used relatively low doses that were sub-cytotoxic for all the compounds in this study. It could be that at higher concentrations, this putative compensatory mechanism is not sufficient anymore to prevent apoptosis. $\mathrm{As}_{2} \mathrm{O}_{3}$, lindane, and OTA have also been found to induce apoptosis in various human and murine cell lines (Battaglia et al., 2010a; Olgun et al., 2004; Qian et al., 2007; Zhou et al., 2005).

The other cluster of processes, which was affected by more than three immunotoxicants (including $\mathrm{As}_{2} \mathrm{O}_{3}, \mathrm{BaP}$ [+ S9], prednisolone, and rapamycin), is negative regulation of biological process and molecular functions (cluster IV, Fig. 1 and Table 2). ID1 and ID2, which encode two important helixloop-helix transcription factors that participate in cell differentiation and proliferation, are among the enriched core genes of this cluster (see the expression patterns of ID1 and ID2 in Fig. 2). The expression of ID1 has been linked with attenuated cytotoxicity induced by $\mathrm{TiO}_{2}$ in lung cells in vitro (Lee et al., 2009). The expression level of ID2 has been found to be suppressed by DEHP in mouse liver in vivo (Wong and Gill, 2002). In T cells, both ID1 and ID2 have been shown to inhibit T cell lineage commitment (Jones-Mason et al., 2012; Wang et al., 2009). ID2 has also been shown to inhibit lymphoid tissue development and haematopoietic stem cell differentiation (Cherrier et al., 2012). Therefore, the compounds that affect the mRNA levels of these genes can potentially alter the differentiation status of lymphoid organs and T-cells.

Both ID1 and ID2 are also among the enriched core genes of cluster II, which comprises the processes related with cell cycle control, regulation of gene transcription and protein translation. This cluster was mainly upregulated by OTA (+ S9 and - S9), and downregulated by lindane (cluster II, Fig. 1 and Table 2). Regulation of transcription and translation is likely associated with cell cycle control (DiPaola, 2002). Due to the high proliferation rate of immune cells, the cell cycle controlling pathways in immune cells might be more sensitive to toxicants than other cell types. Our observations that OTA and lindane both affected the mRNA levels of genes involved in cell cycle regulation are in agreement with previous studies. Induction of cell cycle arrest by OTA has been reported in human PBMCs (Liu et al., 2012). Lindane has also been demonstrated to disrupt cell cycle in various cell lines (Kalantzi et al., 2004). In addition, our observation that OTA affected the genes involved in regulation of transcription and translation is in line with previous studies, in which inhibition of RNA (Dirheimer and Creppy, 1991) and DNA synthesis (Stormer and Lea, 1995) have been linked with OTA-induced toxicity, both in vivo and in vitro.

Metabolism and biosynthesis of cholesterol and lipids were found to be activated by the S1P-receptor antagonist FTY720 and inhibited by the organochlorine insecticide lindane and the organophosphate 
herbicide propanil (cluster V, Fig. 1 and Table 2). Recent murine studies have shown that (1) enrichment of plasma membrane cholesterol drives T cells towards a Th1 phenotype, which indicates a predisposition towards autoimmune and inflammatory diseases (Surls et al., 2012), and (2) deficiency of LXR $\beta$, a key nuclear receptor for cellular cholesterol efflux, results in lymphoid hyperplasia and enhances responses to antigenic challenge (Bensinger et al., 2008). These studies underline the relevance of cholesterol metabolism in maintaining immune system homeostasis. Our results are in line with previous in vivo studies, as FTY720 has been shown to induce hypercholesterolemia in mice (Klingenberg et al., 2007) and propanil exposure has been found to decrease serum cholesterol level in rats (Santillo et al., 1995).

Furthermore, we observed that the mRNA levels of two $G$ protein-coupled receptors (GPCRs), GPR17 (cluster VII) and GPR18 (cluster I) (see the expression patterns of GPR17 and GPR18 in Fig. 2), were altered by various immunotoxicants. These GPCRs are known to be expressed by $T$ cells and play important roles in mediating cellular immune responses. For instance, GPR17 is known to aggravate Th2 immune responses mediated by cysteinyl leukotrienes (Laidlaw and Boyce, 2012), and GPR18 expression correlates with the Th1 phenotype of human T cells (Herrera et al., 2006). Further research is needed to establish whether these GPCRs could also mediate chemically induced immunotoxicity.

Besides the processes that were commonly affected by immunotoxicants, we also identified processes that were affected by one or two of the immunomodulating compounds. Notch and NF-KB signaling pathways were found to be activated by OTA (+ S9 and - S9) (cluster VII, Fig. 1 and Table 2). Notch signaling has been well characterized for its role in promoting $T$ lineage commitment and maturation (Yuan et al., 2010). It also contributes to the peripheral T-cell response as the inhibition of endogenous Notch activation decreases the proliferation of activated T cells via enhancing a positive feedback loop involving IL-2 and its high affinity receptor CD25 (Adler et al., 2003). The importance of NF-KB signaling in the immune system has been well described. In general, NF-KB family members are transcription factors that regulate various aspects of both innate and adaptive immune responses by controlling the transcription of cytokines and other soluble factors that regulate cellular proliferation, differentiation, and survival (Hayden et al., 2006; Vallabhapurapu and Karin, 2009). The NF-kB signaling pathway can be activated by ER stress, increased calcium levels, and oxidative stress (Fardoun et al., 2007; Pahl and Baeuerle, 1996). This pathway is also known to play a role in T cell activation (Katika et al., 2011). NF-KB signaling pathway has already been associated with OTAinduced toxicity both in vivo and in vitro (Ferrante et al., 2008; Kumar et al., 2013; Schwerdt et al., 2007).

Response to retinoic acid was found to be induced by TBTC and TBTO (cluster XIII, Fig. 1 and Table 2). Retinoic acid is known to be a ligand of retinoic acid receptors (RARs) and retinoic $X$ receptors (RXRs) (Pfahl and Chytil, 1996). Three enriched core genes of cluster XIII, ABCA1, ABCG1 and KLF2, are known target genes of the nuclear receptor LXR (Zelcer and Tontonoz, 2006), RAR (Berry et al., 2012), and RXR (Costet et al., 2003). The up-regulation of ABCA1, ABCG1, and KLF2 by TBTC and TBTO indicated the activation of these receptors (see the expression patterns of $A B C A 1$ and $A B C G 1$ 
in Fig. 2). Our results are in line with the recently reported findings of Cui et al., (2011) that TBTC exposure increased $A B C A 1$ mRNA expression by activating $L X R \alpha / R X R$ signaling and thereby modulating cellular efflux of cholesterol in mouse macrophages. Effects of TBTO on lipid metabolism have also been suggested in previous toxicogenomics studies with rodent thymocytes (Baken et al., 2007; van Kol et al., 2012). LXR, RXR, and RAR-signaling have been shown to be a key pathway in controlling immune tolerance and adaptive immune responses (Hall et al., 2011; Kidani and Bensinger, 2012). Another study with TBTO-treated Jurkat cells has demonstrated not only the induction of ER stress and oxidative stress, but also the activation of NF-KB and nuclear factor of activated T cells pathways and the induction of apoptosis (Katika et al., 2011). However, the concentrations of TBTO used in the latter study (200 and 500nM) were higher than the one applied in the present study $(100 \mathrm{nM})$ and might represent the onset of cytotoxicity.

Furthermore, we observed that the mycotoxin OTA (+ S9 and - S9) and the organochlorine insecticide lindane both induced ALDH8A1, a gene encoding an enzyme involved in the biosynthesis of retinoic acid (cluster V, Table 1 and Fig. 2). ALDH8A1 converts 9-cis-retinal into RXR ligand 9-cis-retinoic acid. Presently, it is not known whether $\mathrm{T}$ lymphocytes can produce retinoic acid, although retinoic acid that is produced by dendritic cells or epithelial cells can regulate the differentiation and functionality of $T$ lymphocytes (Hall et al., 2011). Therefore further research is required to verify whether the transcriptional activation of ALDH8A1 by OTA or lindane could result in increased cellular retinoic acid biosynthesis in T lymphocytes, or in other immune cells, and could thereby potentially be involved in OTA or lindane-mediated immunotoxicity.

Most of the biological processes and molecular functions that we identified with Metacore were confirmed by GSEA. Compared to Metacore, GSEA is a more sensitive statistical tool because it uses the full transcriptome data as the input without enforcing a statistical cutoff beforehand. In this way, GSEA allows the detection of subtle effects on the mRNA levels of large groups of genes. GSEA analyses showed that the gene sets related with ribonucleoprotein complex, nucleolus, and mitochondria were commonly affected by direct immunotoxicants (Supplementary fig. 3B). The effects on the ribonucleoprotein complex and the nucleolus are likely related with regulation of transcription and translation, which are among the affected biological processes that were identified by Metacore analyses. Mitochondrial dysfunction has been linked to direct immunotoxicity of a wide range of chemicals, including metals, organotin compounds, dioxins, and immunosuppressive drugs (Baken et al., 2007; Bustamante et al., 2004; Kobayashi et al., 2009; Mitra et al., 2013). Mitochondrial dysfunction is also known to be associated with oxidative stress and apoptosis, both in vivo and in vitro (Gerlach et al., 2007; Yuzefovych et al., 2013). These latter processes were also among the biological processes that were implied by our data.

Some of the pathways and processes that we identified overlap with those described in previous immunotoxicogenomics studies that also aimed at identifying common pathways/genes involved in immunotoxicity, including the processes related with cellular stress responses (response to chemical stimulus, response to unfolded proteins, and response to oxidative stress), and regulation of NF-KB 
signaling (Hochstenbach et al., 2010, 2012; Vandebriel et al., 2010). Response to oxidative stress was also found in other toxicogenomics studies, for instance, for OTA in rat kidney cells in vivo (Lühe et al., 2003) and human liver cells in vitro (Hundhausen et al., 2008), and for $\mathrm{MeHg}$ in fish liver in vivo (Yadetie et al., 2013). Furthermore, our study revealed new processes and pathways that are potentially associated with immunotoxicity, such as modulation of lipid metabolism, retinoic acid signaling, and Notch signaling. Further research is required to validate the relevance of these potential mechanisms.

When comparing our data with the $\mathrm{As}_{2} \mathrm{O}_{3}$, TBTO, and OTA data from other toxicogenomics studies, the degree of overlap between Jurkat and other immune cells was higher than the overlap between Jurkat cells and the liver cell line HepG2, which suggested a cell type dependent effect of the compounds on gene expression (Supplementary fig. 4 to 6 ).

In order to estimate the potential relevance of these effects for immunomodulation in humans in vivo we compared the exposure concentrations that we applied in vitro with circulating concentrations in humans in vivo (Supplementary table 3). This approach showed that for some crucial test compounds, being the immunomodulators $\mathrm{As}_{2} \mathrm{O}_{3}$, DON, TBTC, TBTO, FTY720 and mycophenolic acid and the nonimmunotoxic control $\mathrm{AgNO}_{3}$, furosemide, and azathioprine, the exposure concentrations that we applied in vitro were within the same range as humans in vivo (0.33-3x). For the other test compounds, the concentrations that we used were different from the in vivo situation, as a consequence of the selection criteria for the concentrations ( $\leq \mathrm{CV} 80$ at $24 \mathrm{~h}$ ).

Furthermore, some of the genes we identified overlap with human in vivo data. For instance, we have found an induction of the gene $\mathrm{FBXO} 32$ by $\mathrm{As}_{2} \mathrm{O}_{3}$ (Supplementary fig. 1). A similar effect on $\mathrm{FBXO} 32$ has been found in lymphocytes of human individuals chronically exposed to $\mathrm{As}_{2} \mathrm{O}_{3}$-contaminated drinking water (Andrew et al., 2008). This observation underlines the relevance of the effects on mRNA expression levels that we observed in the present in vitro study for human direct immunotoxicity in vivo.

In conclusion, by applying an in vitro toxicogenomics approach, we have identified cellular pathways and processes that are transcriptionally modulated upon exposure to direct immunotoxicants. The present study has demonstrated the value of the Jurkat $T$ cell line as a sensitive system for identifying mechanisms underlying direct immunotoxicity. Because apparently diverse MOAs are involved, it is envisaged that an assay based on a set of pathways or genes, rather than one single gene, will allow to screen compounds for direct immunotoxicity. Finally, it should be noted that the Jurkat cell model may not be useful to test compounds that specifically target immune cells other than $T$ cells. Therefore, the assay that can be developed based on the results of the present study should not be considered as a universal screening assay but as a promising assay to complement animal free immunotoxicity testing approaches. 


\section{Funding}

Netherlands Genomics Initiative; Netherlands Organisation for Scientific Research; Netherlands Toxicogenomics Centre (05060510); Dutch Technology Foundation Stichting voor de Technische Wetenschappen (MFA6809). 


\section{References}

Adler, S. H., Chiffoleau, E., Xu, L., Dalton, N. M., Burg, J. M., Wells, A. D., Wolfe, M. S., Turka, L. A., and Pear, W. S. (2003). Notch signaling augments T cell responsiveness by enhancing CD25 expression. J. Immunol. 171, 2896-2903.

Al-Anati, L., and Petzinger, E. (2006). Immunotoxic activity of ochratoxin A. J. Vet. Pharmacol. Ther. 29, 79-90.

Alluwaimi, A. M., and Hussein, Y. (2007). Diazinon immunotoxicity in mice: Modulation of cytokines level and their gene expression. Toxicology 236, 123-131.

Andrew, A. S., Jewell, D. A., Mason, R. A., Whitfield, M. I., Moore, J. H., and Karagas, M. R. (2008). Drinking-water arsenic exposure modulates gene expression in human lymphocytes from a U.S. population. Environ. Health Perspect. 116, 524-531.

Ashry, K. M., El-Sayed, Y. S., Khamiss, R. M., and El-Ashmawy, I. M. (2010). Oxidative stress and immunotoxic effects of lead and their amelioration with myrrh (Commiphora molmol) emulsion. Food Chem. Toxicol. 48, 236-241.

Baken, K. A., Arkusz, J., Pennings, J. L., Vandebriel, R. J., and van Loveren, H. (2007). In vitro immunotoxicity of bis(tri-n-butyltin)oxide (TBTO) studied by toxicogenomics. Toxicology 237, 35-48.

Baken, K. A., Pennings, J. L., Jonker, M. J., Schaap, M. M., de Vries, A., van Steeg, H., Breit, T. M., and van Loveren, H. (2008). Overlapping gene expression profiles of model compounds provide opportunities for immunotoxicity screening. Toxicol. Appl. Pharmacol. 226, 46-59.

Ballou, L. M., and Lin, R. Z. (2008). Rapamycin and mTOR kinase inhibitors. J. Chem. Biol. 1, 27-36.

Battaglia, C. L., Gogal, R. M., Jr. Zimmerman, K., and Misra, H. P. (2010a). Malathion, lindane, and piperonyl butoxide, individually or in combined mixtures, induce immunotoxicity via apoptosis in murine splenocytes in vitro. Int. J. Toxicol. 29, 209-220.

Battaglia, C. L., Gogal, R. M., Jr. Zimmerman, K., and Misra, H. P. (2010b). Malathion, lindane, and piperonyl butoxide, individually or in combined mixtures, induce immunotoxicity via apoptosis in murine splenocytes In vitro. Int. J. Toxicol. 29, 209-220.

Bensinger, S. J., Bradley, M. N., Joseph, S. B., Zelcer, N., Janssen, E. M., Hausner, M. A., Shih, R., Parks, J. S., Edwards, P. A., Jamieson, B. D., and Tontonoz, P. (2008). LXR signaling couples sterol metabolism to proliferation in the acquired immune response. Cell 134, 97-111.

Berry, D. C., DeSantis, D., Soltanian, H., Croniger, C. M., and Noy, N. (2012). Retinoic acid upregulates preadipocyte genes to block adipogenesis and suppress diet-induced obesity. Diabetes 61, 1112-1121.

Borchers, A., Teuber, S. S., Keen, C. L., and Gershwin, M. E. (2010). Food Safety. Clin. Prev. Allergy Immunol. 39, 95-141.

Briscoe, V. J., Ertl, A. C., Tate, D. B., and Davis, S. N. (2008). Effects of the selective serotonin reuptake inhibitor, fluoxetine, on counterregulatory responses to hypoglycemia in individuals with T1DM. Diabetes. 57, 3315-3322.

Brouard, S., Puig-Pey, I., Lozano, J. J., Pallier, A., Braud, C., Giral, M., Guillet, M., Londoño, M. C., Oppenheimer, F., Campistol, J. M., Soulillou, J. P., and Sanchez-Fueyo, A. (2010). Comparative 
transcriptional and phenotypic peripheral blood analysis of kidney recipients under cyclosporin $A$ or sirolimus monotherapy. Am. J. Transplant. 10, 2604-2614.

Bustamante, J., Caldas Lopes, E., Garcia, M., Di Libero, E., Alvarez, E., and Hajos, S. E. (2004). Disruption of mitochondrial membrane potential during apoptosis induced by PSC 833 and CsA in multidrug-resistant lymphoid leukemia. Toxicol. Appl. Pharmacol. 199, 44-51.

Carlson, E. A., Li, Y., and Zelikoff, J. T. (2004). Benzo[a]pyrene-induced immunotoxicity in Japanese medaka (Oryzias latipes): relationship between lymphoid CYP1A activity and humoral immune suppression. Toxicol. Appl. Pharmacol. 201, 40-52.

Chakrabarti, A., Chen, A. W., and Varner, J. D. (2011). A review of the mammalian unfolded protein response. Biotechnol. Bioeng. 108, 2777-2793.

Chen, J., Yusuf, I., Andersen, H.M., and Fruman, D. A. (2006). FOXO Transcription Factors Cooperate with $\delta E F 1$ to Activate Growth Suppressive Genes in B Lymphocytes. J. Immunol. 176, 2711-2721.

Chen, Q., Zhang, Z., Zhang, R., Niu, Y., Bian, X. and Zhang, Q. (2011). Tributyltin chloride-induced immunotoxicity and thymocyte apoptosis are related to abnormal Fas expression. Int. J. Hyg. Environ. Health 214, 145-150.

Cherrier, M., Sawa, S., and Eberl, G. (2012). Notch, Id2, and RORyt sequentially orchestrate the fetal development of lymphoid tissue inducer cells. J. Exp. Med. 209, 729-740.

Chou, J. L., Su, H. Y., Chen, L. Y., Liao, Y. P., Hartman-Frey, C., Lai, Y. H., Yang, H. W., Deatherage, D. E., Kuo, C. T., Huang, Y. W., Yan, P. S., Hsiao, S. H., Tai, C. K., Lin, H. J. L., Davuluri, R. V., Chao, T. K., Nephew, K. P., Huang, T. H., Lai, H. C., and Chan, M. W. Y. (2010). Promoter hypermethylation of FBXO32, a novel TGF-[beta]/SMAD4 target gene and tumor suppressor, is associated with poor prognosis in human ovarian cancer. Lab. Invest. 90, 414-425.

Chun, J., and Hartung, H. P. (2010). Mechanism of Action of Oral Fingolimod (FTY720) in Multiple Sclerosis. Clin. Neuropharmacol. 33, 91-101.

Coelho, P., Costa, S., Silva, S., Walter, A., Ranville, J., Sousa, A. C., Costa, C., Coelho, M., GarcíaLestón, J., Pastorinho, M. R., Laffon, B., Pásaro, E., Harrington, C., Taylor, A., and Teixeira, J. P. (2012). Metal(Loid) Levels in Biological Matrices from Human Populations Exposed to Mining Contamination-Panasqueira Mine (Portugal). J. Toxicol. Environ. Health, Part A 75, 893-908.

Corsini, E., and Roggen, E. L. (2009). Immunotoxicology: opportunities for non-animal test development. Altern. Lab. Anim. 37, 387-397.

Corsini, E., Sangiovanni, E., Avogadro, A., Galbiati, V., Viviani, B., Marinovich, M., Galli, C. L., Dell'Agli, M. and Germolec, D. R. (2012a). In vitro characterization of the immunotoxic potential of several perfluorinated compounds (PFCs). Toxicol. Appl. Pharmacol. 258, 248-255.

Corsini, E., Sokooti, M., Galli, C. L., Moretto, A. and Colosio, C. (2012b). Pesticide induced immunotoxicity in humans: A comprehensive review of the existing evidence. Toxicology. 307. 123135.

Costet, P., Lalanne, F., Gerbod-Giannone, M. C., Molina, J. R., Fu, X., Lund, E. G., Gudas, L. J., and Tall, A. R. (2003). Retinoic acid receptor-mediated induction of ABCA1 in macrophages. Mol. Cell. Biol. 23, 7756-7766. 
Cui, H., Okuhira, K., Ohoka, N., Naito, M., Kagechika, H., Hirose, A., and Nishimaki-Mogami, T. (2011). Tributyltin chloride induces ABCA1 expression and apolipoprotein A-I-mediated cellular cholesterol efflux by activating LXRalpha/RXR. Biochem. Pharmacol. 81, 819-824.

Dar, S. A., Das, S., Ramachandran, V. G., Bhattacharya, S. N., Mustafa, M. D., Banerjee, B. D., and Verma, P. (2012). Alterations in T-lymphocyte sub-set profiles and cytokine secretion by PBMC of systemic lupus erythematosus patients upon in vitro exposure to organochlorine pesticides. $J$. Immunotoxicol. 9, 85-95.

Descotes, J. (2005). Immunotoxicology: role in the safety assessment of drugs. Drug Saf. 28, 127136.

DiPaola, R. S. (2002). To Arrest or Not To G2-M Cell-Cycle Arrest. Clin. Cancer Res. 8, 3311-3314.

Dirheimer, G. and Creppy, E. (1991). Mechanism of action of ochratoxin A. IARC Sci. Publ. 115, 171186.

Dombroski, B. A., Nayak, R. R., Ewens, K. G., Ankener, W., Cheung, V. G., and Spielman, R. S. (2010). Gene expression and genetic variation in response to endoplasmic reticulum stress in human cells. Am. J. Hum. Genet. 86, 719-729.

Duranteau, J., Chandel, N. S., Kulisz, A., Shao, Z., and Schumacker, P. T. (1998). Intracellular Signaling by Reactive Oxygen Species during Hypoxia in Cardiomyocytes. J. Biol. Chem. 273, 1161911624.

Eisen, M. B., Spellman, P. T., Brown, P. O., and Botstein, D. (1998). Cluster analysis and display of genome-wide expression patterns. Proc. Nat. Acad. Sci. U.S.A. 95, 14863-14868.

Ekhart, C., Rodenhuis, S., Beijnen, J., and Huitema, A. (2009). Carbamazepine induces bioactivation of cyclophosphamide and thiotepa. Cancer Chemother. Pharmacol. 63, 543-547.

Ekins, S., Bugrim, A., Brovold, L., Kirillov, E., Nikolsky, Y., Rakhmatulin, E., Sorokina, S., Ryabov, A., Serebryiskaya, T., Melnikov, A., Metz, J., and Nikolskaya, T. (2006). Algorithms for network analysis in systems-ADME/Tox using the MetaCore and MetaDrug platforms. Xenobiotica 36, 877-901.

Fakata, K. L., Elmquist, W. F., Swanson, S. A., Vorce, R. L., Prince, C., and Stemmer, P. M. (1998). Cyclosporin a has low potency as a calcineurin inhibitor in cells expressing high levels of $\mathrm{P}$ glycoprotein. Life Sci. 62, 2441-2448.

Fardoun, R. Z., Asghar, M., and Lokhandwala, M. (2007). Role of nuclear factor kappa B (NF-kB) in oxidative stress-induced defective dopamine D1 receptor signaling in the renal proximal tubules of Sprague-Dawley rats. Free Radic. Biol. Med. 42, 756-764.

Ferrante, M. C., Raso, G. M., Bilancione, M., Esposito, E., lacono, A., and Meli, R. (2008). Differential modification of inflammatory enzymes in J774A.1 macrophages by ochratoxin $A$ alone or in combination with lipopolysaccharide. Toxicol. Lett. 181, 40-46.

Food and Drug Administration. (2006). International Conference on Harmonisation; Guidance on S8 Immunotoxicity Studies for Human Pharmaceuticals; availability. Notice. Fed. Regist. 71, 1919319194.

Frawley, R., White, K., Jr. Brown, R., Musgrove, D., Walker, N., and Germolec, D. (2011). Gene Expression Alterations in Immune System Pathways in the Thymus after Exposure to Immunosuppressive Chemicals. Environ. Health. Perspect. 119, 371-376. 
Galbiati, V., Mitjans, M., and Corsini, E. (2010). Present and future of in vitro immunotoxicology in drug development. J. Immunotoxicol. 7, 255-267.

Galluzzi, L., De Santi, M., Crinelli, R., De Marco, C., Zaffaroni, N., Duranti, A., Brandi, G., and Magnani, M. (2012). Induction of Endoplasmic Reticulum Stress Response by the Indole-3-Carbinol Cyclic Tetrameric Derivative CTet in Human Breast Cancer Cell Lines. PLoS ONE 7, e43249.

Gao, S., Zhou, J., Zhao, Y., Toselli, P. and Li, W. (2012). Hypoxia Response Element (HRE)-directed Transcriptional Regulation of the Rat Lysyl Oxidase Gene in Response to Cobalt and Cadmium. Toxicol. Sci. 132, 379-389.

Gerlach, M., Deckert, J., Double, K., Koutsilieri, E., Leuner, K., Pantel, J., Frey, C., Schindowski, K., Schulz, K., Wegat, T., Maurer, K., Eckert, A., and Müller, W. E. (2007). Enhanced apoptosis, oxidative stress and mitochondrial dysfunction in lymphocytes as potential biomarkers for Alzheimer's disease. pp. 207-215. Springer, Vienna, Austria.

Gill, H. S., Grammatopoulos, G., Adshead, S., Tsialogiannis, E., and Tsiridis, E. (2012). Molecular and immune toxicity of $\mathrm{CoCr}$ nanoparticles in MoM hip arthroplasty. Trends Mol. Med. 18, 145-155.

Gómez, S. E., del Razo, L. M., and Muñoz Sanchez, J. L. (2005). Induction of DNA damage by free radicals generated either by organic or inorganic arsenic (AsIII, MMAIII, and DMAIII) in cultures of B and T lymphocytes. Biol. Trace Elem, Res. 108, 115-126.

Greijer, A. E., van der Groep, P., Kemming, D., Shvarts, A., Semenza, G. L., Meijer, G. A., van de Wiel, M. A., Belien, J. A. M., van Diest, P. J., and van der Wall, E. (2005). Up-regulation of gene expression by hypoxia is mediated predominantly by hypoxia-inducible factor 1 (HIF-1). J. Pathol. 206, 291-304.

Griesmacher, A., Weigel, G., Seebacher, G., and Müller, M. M. (1997). IMP-dehydrogenase inhibition in human lymphocytes and lymphoblasts by mycophenolic acid and mycophenolic acid glucuronide. Clin. Chem. 43, 2312-2317.

Guillemin, K., and Krasnow, M. A. (1997). The Hypoxic Response: Huffing and HIFing. Cell 89, 9-12. Hall, J. A., Grainger, J. R., Spencer, S. P., and Belkaid, Y. (2011). The Role of Retinoic Acid in Tolerance and Immunity. Immunity 35, 13-22.

Hannam, M. L., Bamber, S. D., John Moody, A., Galloway, T. S., and Jones, M. B. (2010). Immunotoxicity and oxidative stress in the Arctic scallop Chlamys islandica: Effects of acute oil exposure. Ecotoxicol. Environ. Saf. 73, 1440-1448.

Hayden, M. S., West, A. P., and Ghosh, S. (2006). NF-kappaB and the immune response. Oncogene, 25, 6758-6780.

$\mathrm{He}, \mathrm{X}$., and Ma, Q. (2009). Induction of metallothionein I by arsenic via metal-activated transcription factor 1: critical role of c-terminal cysteine redidues in arsenic sensing. J. Biol. Chem. 284, 1260912621.

Herrera, J., Guenther, C., Lametschwandtner, G., and Werner, G. (2006). GPR18 as a biomarker for TH1 mediated immune response. G01N33/50 ed. Novartis AG, Novatis-Pharma Gmbh.

Hochstenbach, K., van Leeuwen, D., Gmuender, H., Gottschalk, R., Stølevik, S. B., Nygaard, U. C., Lovik, M., Granum, B., Namork, E., Meltzer, H. M., Kleinjans, J., van Delft, J. H., and van Loveren, H. 
(2012). Toxicogenomic profiles in relation to maternal immunotoxic exposure and immune functionality in newborns. Toxicol. Sci. 129, 315-324.

Hochstenbach, K., van Leeuwen, D. M., Gmuender, H., Stølevik, S. B., Nygaard, U. C., Løvik, M., Granum, B., Namork, E., van Delft, J. H., and van Loveren, H. (2010). Transcriptomic Profile Indicative of Immunotoxic Exposure: In Vitro Studies in Peripheral Blood Mononuclear Cells. Toxicol. Sci. 118, 19-30.

Hollnagel, A., Oehlmann, V., Heymer, J., Rüther, U., and Nordheim, A. (1999). Id genes are direct targets of bone morphogenetic protein induction in embryonic stem cells. J. Biol. Chem. 274, 1983819845.

Hundhausen, C., Boesch-Saadatmandi, C., Matzner, N., Lang, F., Blank, R., Wolffram, S., Blaschek, W., and Rimbach, G. (2008). Ochratoxin A lowers mRNA levels of genes encoding for key proteins of liver cell metabolism. Cancer Genomics Proteomics 5, 319-332.

Institoris, L., Siroki, O., Desi, I., Lesznyak, J., Serenyi, P., Szekeres, E., and Petri, I. (1998). Extension of the protocol of OECD guideline 407 (28-day repeated dose oral toxicity test in the rat) to detect potential immunotoxicity of chemicals. Hum. Exp. Toxicol. 17, 206-211.

Jakobsen, C. H., Størvold, G. L., Bremseth, H., Follestad, T., Sand, K., Mack, M., Olsen, K. S., Lundemo, A. G., Iversen, J. G., Krokan, H. E., and Schønberg, S. A. (2008). DHA induces ER stress and growth arrest in human colon cancer cells: associations with cholesterol and calcium homeostasis. J. Lipid Res. 49, 2089-2100.

Jo, H., Choe, S. S., Shin, K. C., Jang, H., Lee, J. H., Seong, J. K., Back, S. H. and Kim, J. B. (2012). Endoplasmic reticulum stress induces hepatic steatosis via increased expression of the hepatic very low-density lipoprotein receptor. Hepatology, 57, 1366-1377.

Jones-Mason, M. E., Zhao, X., Kappes, D., Lasorella, A., lavarone, A., and Zhuang, Y. (2012). E protein transcription factors are required for the development of CD4(+) lineage T Cells. Immunity 36, 348-361.

Kalantzi, O. I., Hewitt, R., Ford, K. J., Cooper, L., Alcock, R. E., Thomas, G. O., Morris, J. A., McMillan, T. J., Jones, K. C., and Martin, F. L. (2004). Low dose induction of micronuclei by lindane. Carcinogenesis 25, 613-622.

Katika, M. R., Hendriksen, P. J. M., de Ruijter, N. C., van Loveren, H., and Peijnenburg, A. (2012a). Immunocytological and biochemical analysis of the mode of action of bis (tri-n-butyltin) tri-oxide (TBTO) in Jurkat cells. Toxicol. Lett. 212, 126-136.

Katika, M. R., Hendriksen, P. J., Shao, J., van Loveren, H., and Peijnenburg, A. (2012b). Transcriptome analysis of the human $\mathrm{T}$ lymphocyte cell line Jurkat and human peripheral blood mononuclear cells exposed to deoxynivalenol (DON): New mechanistic insights. Toxicol. Appl. Pharmacol. 264, 51-64.

Katika, M. R., Hendriksen, P. J., van Loveren, H., and Peijnenburg, A. (2011). Exposure of Jurkat cells to bis (tri-n-butyltin) oxide (TBTO) induces transcriptomics changes indicative for ER- and oxidative stress, T cell activation and apoptosis. Toxicol. Appl. Pharmacol. 254, 311-322.

Katoh, M., and Katoh, M. (2009). Transcriptional regulation of WNT2B based on the balance of Hedgehog, Notch, BMP and WNT signals. Int. J. Oncol. 34, 1411-1415. 
Kaufman, R. J., Scheuner, D., Schroder, M., Shen, X., Lee, K., Liu, C. Y., and Arnold, S. M. (2002). The unfolded protein response in nutrient sensing and differentiation. Nat. Rev. Mol. Cell Biol. 3, 411421.

Kawata, K., Yokoo, H., Shimazaki, R., and Okabe, S. (2007). Classification of heavy-metal toxicity by human DNA microarray analysis. Environ. Sci. Technol. 41, 3769-3774.

Kidani, Y., and Bensinger, S. J. (2012). Liver $X$ receptor and peroxisome proliferator-activated receptor as integrators of lipid homeostasis and immunity. Immunol. Rev. 249, 72-83.

Klingenberg, R., Nofer, J.-R., Rudling, M., Bea, F., Blessing, E., Preusch, M., Grone, H. J., Katus, H. A., Hansson, G. K., and Dengler, T. J. (2007). Sphingosine-1-Phosphate Analogue FTY720 Causes Lymphocyte Redistribution and Hypercholesterolemia in ApoE-Deficient Mice. Arterioscler. Thromb. Vasc. Biol. 27, 2392-2399.

Kobayashi, D., Ahmed, S., Ishida, M., Kasai, S., and Kikuchi, H. (2009). Calcium/calmodulin signaling elicits release of cytochrome c during 2,3,7,8-tetrachlorodibenzo-p-dioxin-induced apoptosis in the human lymphoblastic T-cell line, L-MAT. Toxicology 258, 25-32.

Koch, H. M., Preuss, R., and Angerer, J. (2006). Di(2-ethylhexyl)phthalate (DEHP): human metabolism and internal exposure - an update and latest results1. Int. J. Androl. 29, 155-165.

Kumar, R., Alam, S., Chaudhari, B. P., Dwivedi, P. D., Jain, S. K., Ansari, K. M., and Das, M. (2013). Ochratoxin A-induced cell proliferation and tumor promotion in mouse skin by activating the expression of cyclin-D1 and cyclooxygenase-2 through nuclear factor-kappa B and activator protein-1. Carcinogenesis 34, 647-657.

Laidlaw, T. M., and Boyce, J. A. (2012). Cysteinyl leukotriene receptors, old and new; implications for asthma. Clin. Exp. Allergy 42, 1313-1320.

Lankveld, D. P., van Loveren, H., Baken, K. A., Vandebriel, R. J., and Dietert, R. R. (2010). In vitro testing for direct immunotoxicity: state of the art immunotoxicity testing (J. M. Walker, Ed.), pp. 401423. Humana Press: NY, USA.

Lee, Y. S., Yoon, S., Yoon, H. J., Lee, K., Yoon, H. K., Lee, J. H., and Song, C. W. (2009). Inhibitor of differentiation 1 (Id1) expression attenuates the degree of TiO2-induced cytotoxicity in H1299 nonsmall cell lung cancer cells. Toxicol. Lett. 189, 191-199.

Levin, N., Bischoff, E. D., Daige, C. L., Thomas, D., Vu, C. T., Heyman, R. A., Tangirala, R. K., and Schulman, I. G. (2005). Macrophage liver $X$ receptor is required for antiatherogenic activity of LXR agonists. Arterioscler. Thromb. Vasc. Biol. 25, 135-142.

Liu, J., Liu, Y., Habeebu, S. S., and Klaassen, C. D. (1999). Metallothionein-null mice are highly susceptible to the hematotoxic and immunotoxic effects of chronic $\mathrm{CdCl} 2$ exposure. Toxicol. Appl. Pharmacol. 159, 98-108.

Liu, J., Wang, Y., Cui, J., Xing, L., Shen, H., Wu, S., Lian, H., Wang, J., Yan, X., and Zhang, X. (2012). Ochratoxin $A$ induces oxidative DNA damage and $\mathrm{G} 1$ phase arrest in human peripheral blood mononuclear cells in vitro. Toxicol. Lett. 211, 164-171.

Luebke, R. W., Holsapple, M. P., Ladics, G. S., Luster, M. I., Selgrade, M., Smialowicz, R. J., Woolhiser, M. R., and Germolec, D. R. (2006). Immunotoxicogenomics: the potential of genomics technology in the immunotoxicity risk assessment process. Toxicol. Sci. 94, 22-27. 
Lühe, A., Hildebrand, H., Bach, U., Dingermann, T., and Ahr, H. J. (2003). A new approach to studying ochratoxin A (OTA)-induced nephrotoxicity: expression profiling in vivo and in vitro employing cDNA microarrays. Toxicol. Sci. 73, 315-328.

Maccubbin, A. E., Caballes, L., Riordan, J. M., Huang, D. H., and Gurtoo, H. L. (1991). A cyclophosphamide/DNA phosphoester adduct formed in vitro and in vivo. Cancer Res. 51, 886-892.

Madathil, B. K., Lin, Q., Hew, C. L., and Mohanty, M. (2010). Hypoxia-like effect of Cobalt Chromium alloy micro particles on fibroblasts in vitro. J. Orthop. Res. 28, 1360-1367.

Magkoufopoulou, C., Claessen, S. M., Tsamou, M., Jennen, D. G., Kleinjans, J. C., and van Delft, J. H. M. (2012). A transcriptomics-based in vitro assay for predicting chemical genotoxicity in vivo. Carcinogenesis 33, 1421-1429.

Manderville, R. A. (2005). A case for the genotoxicity of ochratoxin A by bioactivation and covalent DNA adduction. Chem. Res. Toxicol. 18, 1091-1097.

Martini, F., Fernández, C., Tarazona, J. V., and Pablos, M. V. (2012). Gene expression of heat shock protein 70 , interleukin-1 $\beta$ and tumor necrosis factor $\alpha$ as tools to identify immunotoxic effects on Xenopus laevis: A dose-response study with benzo[a]pyrene and its degradation products. Environ. Pollut. 160, 28-33.

Matulis, S. M., Morales, A. A., Yehiayan, L., Croutch, C., Gutman, D., Cai, Y., Lee, K. P., and Boise, L. $H$. (2009). Darinaparsin induces a unique cellular response and is active in an arsenic trioxideresistant myeloma cell line. Mol. Cancer Ther. 8, 1197-1206.

Milićević, D. R., Škrinjar, M., and Baltić, T. (2010). Real and perceived risks for mycotoxin contamination in foods and feeds: challenges for food safety control. Toxins (Basel) 2, 572-592.

Mishra, P. K., Panwar, H., Bhargava, A., Gorantla, V. R., Jain, S. K., Banerjee, S., and Maudar, K. K. (2008). Isocyanates induces DNA damage, apoptosis, oxidative stress, and inflammation in cultured human lymphocytes. J. Biochem. Mol. Toxicol. 22, 429-440.

Mitra, S., Keswani, T., Ghosh, N., Goswami, S., Datta, A., Das, S., Maity, S., and Bhattacharyya, A. (2013). Copper induced immunotoxicity promote differential apoptotic pathways in spleen and thymus. Toxicology 306, 74-84.

Moore, B. A., Jevons, S., and Brammer, K. W. (1979). Inhibition of transpeptidase activity in escherichia coli by thienamycin. Antimicrob. Agents Chemother. 15, 831-833.

Mungrue, I. N., Pagnon, J., Kohannim, O., Gargalovic, P. S., and Lusis, A. J. (2009). CHAC1/MGC4504 is a novel proapoptotic component of the unfolded protein response, downstream of the ATF4-ATF3-CHOP cascade. J. Immunol. 182, 466-476.

Newton, R. (2000). Molecular mechanisms of glucocorticoid action: what is important? Thorax $\mathbf{5 5}$, 603-613.

Olgun, S., Gogal Jr, R. M., Adeshina, F., Choudhury, H., and Misra, H. P. (2004). Pesticide mixtures potentiate the toxicity in murine thymocytes. Toxicology 196, 181-195.

Otsuka, F., Ohno, S., Suzuki, K., Takahashi, K., Ohsawa, M., and Koizumi, S. (2007). Mechanism of metallothionein gene activation mediated by heavy-metal dependent transcription factor MTF-1. Yakugaku Zasshi 127, 675-684. 
Pahl, H. L., and Baeuerle, P. A. (1996). Activation of NF-kB by ER stress requires both $\mathrm{Ca2+}$ and reactive oxygen intermediates as messengers. FEBS Lett. 392, 129-136.

Pestka, J. J. (2010). Deoxynivalenol: mechanisms of action, human exposure, and toxicological relevance. Arch. Toxicol. 84, 663-679.

Pfahl, M., and Chytil, F. (1996). Regulation of Metabolism by Retinoic Acid and its Nuclear Receptors. Annu. Rev. Nutr. 16, 257-283.

Qian, W., Liu, J., Jin, J., Ni, W., and Xu, W. (2007). Arsenic trioxide induces not only apoptosis but also autophagic cell death in leukemia cell lines via up-regulation of Beclin-1. Leuk. Res. 31, 329-339. Rosado-Berrios, C. A., Vélez, C., and Zayas, B. (2011). Mitochondrial permeability and toxicity of diethylhexyl and monoethylhexyl phthalates on TK6 human lymphoblasts cells. Toxicol. In Vitro 25, 2010-2016.

Sakakura, Y., Sato, H., Shiiya, A., Tamba, M., Sagara, J. I., Matsuda, M., Okamura, N., Makino, N., and Bannai, S. (2007). Expression and function of cystine/glutamate transporter in neutrophils. J. Leukoc. Biol. 81, 974-982.

Salazar, K. D., Ustyugova, I. V., Brundage, K. M., Barnett, J. B., and Schafer, R. (2008). A Review of the Immunotoxicity of the Pesticide 3,4-Dichloropropionanalide. J. Toxicol. Environ. Health B Crit. Rev. 11, 630-645.

Santillo, M., Rippa, C., Morte, R. D., Villani, G. R., Santangelo, F., Staiano, N.. and Mondola, P. (1995). Enhancement of tissue lipoperoxidation in propanil-treated rats. Toxicol. Lett. 78, 215-218.

Schäfer, M., Dütsch, S., auf dem Keller, U., Navid, F., Schwarz, A., Johnson, D. A., Johnson, J. A., and Werner, S. (2010). Nrf2 establishes a glutathione-mediated gradient of UVB cytoprotection in the epidermis. Genes Dev. 24, 1045-1058.

Schlezinger, J. J., Howard, G. J., Hurst, C. H., Emberley, J. K., Waxman, D. J., Webster, T., and Sherr, D. H. (2004). Environmental and Endogenous Peroxisome Proliferator-Activated Receptor $Y$ Agonists Induce Bone Marrow B Cell Growth Arrest and Apoptosis: Interactions between Mono(2ethylhexyl)phthalate, 9-cis-Retinoic Acid, and 15-Deoxy- $\Delta$ 12,14-prostaglandin J2. J. Immunol. 173, 3165-3177.

Schröder, M., and Kaufman, R. J. (2005). The mammalian unfolded protein response. Annu. Rev. Biochem. 74, 739-789.

Schwerdt, G., Holzinger, H., Sauvant, C., Königs, M., Humpf, H. U., and Gekle, M. (2007). Long-term effects of ochratoxin A on fibrosis and cell death in human proximal tubule or fibroblast cells in primary culture. Toxicology 232, 57-67.

Shenker, B. J., Pankoski, L., Zekavat, A., and Shapiro, I. M. (2004). Mercury-induced apoptosis in human lymphocytes: caspase activation is linked to redox status. Antioxid. Redox Signal. 4, 379-389.

Shimizu, Y., Nakatsuru, Y., Ichinose, M., Takahashi, Y., Kume, H., Mimura, J., Fujii-Kuriyama, Y., and Ishikawa, T. (2000). Benzo[a]pyrene carcinogenicity is lost in mice lacking the aryl hydrocarbon receptor. Proc. Natl. Acad. Sci. U.S.A. 97, 779-782.

Sikalidis, A., Lee, J. I., and Stipanuk, M. (2011). Gene expression and integrated stress response in HepG2/C3A cells cultured in amino acid deficient medium. Amino Acids 41, 159-171. 
Slotkin, T. A., and Seidler, F. J. (2008). Oxidative and excitatory mechanisms of developmental neurotoxicity: transcriptional profiles for chlorpyrifos, diazinon, dieldrin, and divalent nickel in PC12 cells. Environ. Health Perspect. 117, 587-596.

Snoeij, N. J., Penninks, A. H., and Seinen, W. (1988). Dibutyltin and tributyltin compounds induce thymus atrophy in rats due to a selective action on thymic lymphoblasts. Int. J. Immunopharmacol. 10, 891-899.

Song, S., Kole, S., Precht, P., Pazin, M. J., and Bernier, M. (2010). Activation of heat shock factor 1 plays a role in pyrrolidine dithiocarbamate-mediated expression of the co-chaperone BAG3. Int. J. Biochem. Cell Biol. 42, 1856-1863.

Stenger, C., Naves, T., Verdier, M., and Ratinaud, M. H. (2011). The cell death response to the ROS inducer, cobalt chloride, in neuroblastoma cell lines according to p53 status. Int. J. Oncol. 39, 601-609. Stormer, F. C., and Lea, T. (1995). Effects of ochratoxin A upon early and late events in human T-cell proliferation. Toxicology 95, 45-50.

Subramanian, A., Tamayo, P., Mootha, V. K., Mukherjee, S., Ebert, B. L., Gillette, M. A., Paulovich, A., Pomeroy, S. L., Golub, T. R., Lander, E. S., and Mesirov, J. P. (2005). Gene set enrichment analysis: A knowledge-based approach for interpreting genome-wide expression profiles. Proc. Natl. Acad. Sci. U.S.A. 102, 15545-15550.

Suh, M., Ariese, F., Small, G. J., Jankowiak, R., Hewer, A., and Phillips, D. H. (1995). Formation and persistence of benzo[a]pyrene-DNA adducts in mouse epidermis in vivo: importance of adduct conformation. Carcinogenesis 16, 2561-2569.

Surls, J., Nazarov-Stoica, C., Kehl, M., Olsen, C., Casares, S., and Brumeanu, T. D. (2012). Increased Membrane Cholesterol in Lymphocytes Diverts T-Cells toward an Inflammatory Response. PLoS One 7, e38733.

Takahashi, N., Chernavvsky, D. R., Gomez, R. A., Igarashi, P., Gitelman, H. J., and Smithies, O. (2000). Uncompensated polyuria in a mouse model of Bartter's syndrome. Proc. Natl. Acad. Sci. U.S.A. 97, 5434-5439.

Tan, J., Yang, X., Zhuang, L., Jiang, X., Chen, W., Lee, P. L., Karuturi, R. K., Tan, P. B., Liu, E. T., and Yu, Q. (2007). Pharmacologic disruption of Polycomb-repressive complex 2-mediated gene repression selectively induces apoptosis in cancer cells. Genes Dev. 21, 1050-1063.

Taylor, G. M., Raghuwanshi, S. K., Rowe, D. T., Wadowsky, R. M., and Rosendorff, A. (2011). Endoplasmic reticulum stress causes EBV lytic replication. Blood 118, 5528-5539.

Tchounwou, P. B., Ayensu, W. K., Ninashvili, N., and Sutton, D. (2003). Environmental exposure to mercury and its toxicopathologic implications for public health. Environ. Toxicol. 18, 149-175.

Tontonoz, P., and Mangelsdorf, D. J. (2003). Liver X receptor signaling pathways in cardiovascular disease. Mol. Endocrinol. 17, 985-993.

Treiman, M., Caspersen, C., and Christensen, S. B. (1998). A tool coming of age: thapsigargin as an inhibitor of sarco-endoplasmic reticulum Ca2+-ATPases. Trends Pharmacol. Sci. 19, 131-135.

Usuki, F., Fujita, E., and Sasagawa, N. (2008). Methylmercury activates ASK1/JNK signaling pathways, leading to apoptosis due to both mitochondria- and endoplasmic reticulum (ER)-generated processes in myogenic cell lines. Neurotoxicology 29, 22-30. 
Vallabhapurapu, S., and Karin, M. (2009). Regulation and Function of NF-kB Transcription Factors in the Immune System. Annu. Rev. Immunol. 27, 693-733.

van Kol, S. W., Hendriksen, P. J., van Loveren, H., and Peijnenburg, A. (2012). Transcriptomics analysis of primary mouse thymocytes exposed to bis(tri-n-butyltin)dioxide (TBTO). Toxicology 296, 37-47.

Vandebriel, R. J., Pennings, J. L., Baken, K. A., Pronk, T. E., Boorsma, A., Gottschalk, R., and Van Loveren, H. (2010). Keratinocyte Gene Expression Profiles Discriminate Sensitizing and Irritating Compounds. Toxicol. Sci. 117, 81-89.

Veraldi, A., Costantini, A. S., Bolejack, V., Miligi, L., Vineis, P., and van Loveren, H. (2006). Immunotoxic effects of chemicals: A matrix for occupational and environmental epidemiological studies. Am. J. Ind. Med. 49, 1046-1055.

Wang, G., Hazra, T. K., Mitra, S., Lee, H. M., and Englander, E. W. (2000). Mitochondrial DNA damage and a hypoxic response are induced by $\mathrm{CoCl}(2)$ in rat neuronal $\mathrm{PC} 12$ cells. Nucleic Acids Res. 28, 2135-2140.

Wang, H. C., Perry, S. S., and Sun, X. H. (2009). Id1 attenuates notch signaling and impairs T-cell commitment by elevating deltex1 expression. Mol. Cell. Biol. 29, 4640-4652.

Wang, Y., Wimmer, U., Lichtlen, P., Inderbitzin, D., Stieger, B., Meier, P. J., Hunziker, L., Stallmach, T., Forrer, R., Rulicke, T., Georgiev, O., and Schaffner, W. (2004). Metal-responsive transcription factor-1 (MTF-1) is essential for embryonic liver development and heavy metal detoxification in the adult liver. FASEB J. 18, 1071-1079.

Weinreich, M. A., Takada, K., Skon, C., Reiner, S. L., Jameson, S. C., and Hogquist, K. A. (2009). KLF2 transcription-factor deficiency in $\mathrm{T}$ cells results in unrestrained cytokine production and upregulation of bystander chemokine receptors. Immunity 31, 122-130.

Wong, J. S., and Gill, S. S. (2002). Gene expression changes induced in mouse liver by di(2ethylhexyl) phthalate. Toxicol. Appl. Pharmacol. 185, 180-196.

Yadetie, F., Karlsen, O. A., Lanzén, A., Berg, K., Olsvik, P., Hogstrand, C., and Goksøyr, A. (2013). Global transcriptome analysis of Atlantic cod (Gadus morhua) liver after in vivo methylmercury exposure suggests effects on energy metabolism pathways. Aquat. Toxicol. 126, 314-325.

Yen, Y. P., Tsai, K. S., Chen, Y. W., Huang, C. F., Yang, R. S. and Liu, S. H. (2012). Arsenic induces apoptosis in myoblasts through a reactive oxygen species-induced endoplasmic reticulum stress and mitochondrial dysfunction pathway. Arch. Toxicol. 86, 923-933.

Yin, X., Knecht, D. A., and Lynes, M. A. (2005). Metallothionein mediates leukocyte chemotaxis. BMC Immunol. 6, 21.

Yuan, J. S., Kousis, P. C., Suliman, S., Visan, I., and Guidos, C. J. (2010). Functions of notch signaling in the immune system: consensus and controversies. Annu. Rev. Immunol. 28, 343-365.

Yuzefovych, L. V., Musiyenko, S. I., Wilson, G. L., and Rachek, L. I. (2013). Mitochondrial DNA damage and dysfunction, and oxidative stress are associated with endoplasmic reticulum stress, protein degradation and apoptosis in high fat diet-induced insulin resistance mice. PLoS One 8, e54059. 
Zelcer, N., and Tontonoz, P. (2006). Liver $\mathrm{X}$ receptors as integrators of metabolic and inflammatory signaling. J. Clin. Invest. 116, 607-614.

Zhou, L., Jing, Y., Styblo, M., Chen, Z., and Waxman, S. (2005). Glutathione-S-transferase pi inhibits As2O3-induced apoptosis in lymphoma cells: involvement of hydrogen peroxide catabolism. Blood 105, 1198-1203. 


\section{CHAPTER 3}

\section{Protein phosphorylation profiling identifies potential mechanisms for direct immunotoxicity}

Jia Shao, Inge Stout, Peter J. M. Hendriksen, Henk van Loveren Ad A. C. M. Peijnenburg, and Oscar L. Volger

Submitted for publication 


\begin{abstract}
Signalling networks are essential elements that are involved in diverse cellular processes. One group of fundamental components in various signalling pathways concerns protein tyrosine kinases (PTKs). Various toxicants have been demonstrated to exert their toxicity via modulation of tyrosine kinase activity. The present study aimed to identify common cellular signaling pathways that are involved in chemical-induced direct immunotoxicity. To this end, an antibody array-based profiling approach was applied in order to assess the effects of five immunotoxicants, two immunosuppressive drugs, and two nonimmunotoxic control chemicals on the phosphorylation of 28 receptor tyrosine kinases and 11 crucial signalling nodes in Jurkat T cells. The phosphorylation of ribosomal protein S6 (RPS6), and of kinases Akt, Src, and p44/42 was found to be commonly regulated by immunotoxicants and/or immunosuppressive drugs (at least three compounds), with the largest effect observed upon RPS6. Flow cytometry and western blotting were used to further examine the effect of the model immunotoxicant TBTO on the components of the mTOR-p70S6K-RPS6 pathway. These analyses revealed that both TBTO and the mTOR inhibitor rapamycin inactivate RPS6 but via different mechanisms. Finally, comparison of the protein phosphorylation data to previously obtained transcriptome data of TBTO-treated Jurkat cells resulted in a good correlation at the pathway level and indicated that TBTO affects ribosome biogenesis and leukocyte migration. The effect of TBTO on the latter process was confirmed using a CXCL12 chemotaxis assay.
\end{abstract}




\section{Introduction}

Signalling networks are important elements involved in diverse cellular processes, such as appropriate responses to the extracellular environment, inter- and intracellular communication, metabolism, and cell cycle regulation. Errors in intra-/ intercellular communication give rise to certain diseases such as cancer, diabetes, and autoimmunity (Berg et al., 2002). Protein tyrosine kinases (PTKs), enzymes that catalyse the transfer of phosphate groups from ATP to tyrosine residues of their protein substrates, are fundamental components of diverse signaling pathways. In the human genome, 58 tyrosine kinases are identified as receptor types (RTKs) and 32 are non-receptor types (nRTKs) (Robinson et al., 2000).

PTKs are of widespread interest not only because of their intrinsic physiological functions, but also their roles in the toxicity of various immunotoxicants. For instance, the immunotoxic effects of benzo[a]pyrene has been linked to activation of the nRTK Fyn kinase and ZAP-70 kinases in human HPB-ALL T cells (Davila, 1999). The heavy metal mercuric chloride $\left(\mathrm{HgCl}_{2}\right)$ has been shown to exert its immunotoxic effects via activation of Src-family kinases such as Hck (nRTK) in human hematopoietic cells (Robbins et al., 2000). PTKs have also been of interest as drug targets in the treatment of malignant diseases such as cancer. For instance, the antibodies trastuzumab (antiErbB2) and cetuximab (anti-EFGR) are used in treatment of ErbB2 ${ }^{+}$breast cancer and EGFR ${ }^{+}$ colorectal cancer, respectively (Tworkoski et al., 2011). Regarding immunological disorders, activation of ephrin receptors, for instance the EphA4 and EphB2 receptors, have been linked with autoimmune diseases (Mikasova et al., 2012; Munro et al., 2013).

In a previous study, we assessed the effects of a wide range of compounds on the transcriptome of the human Jurkat T-cell line in vitro with the aim to identify common mechanisms underlying direct immunotoxicity (Shao et al., 2013). Several general processes including ER stress, oxidative stress, regulation of cell cycle, and anti-apoptosis were found to be affected by immunotoxicants in common (Shao et al., 2013). The present work sought to identify cellular signalling pathways that are commonly modulated by immunotoxicants at the protein phosphorylation level, and to connect these signalling pathways to cellular functions, thereby defining potentially novel modes of action of direct immunotoxicants. For this purpose, Jurkat cells were exposed to a subset of the immunotoxicants used in the previous transcriptomics study, i.e., the immunotoxicants lindane, ochratoxin A, TBTC, TBTO, and DON, the immunosuppressive drugs rapamycin and mycophenolic acid, and the nonimmunotoxicants urethane and mannitol. Upon treatment of the Jurkat cells, the effects on phosphorylation of a set of 39 signalling proteins were assessed using receptor tyrosine kinase (RTK) antibody arrays. This set included 28 RTKs and 11 proteins belonging to other important signalling networks.

Our results suggested that various immunotoxicants affect the phosphorylation of ribosomal protein S6 (RPS6) in human T cells, which is an important downstream effector of the mTOR pathway. Further investigation into this pathway with the model immunotoxicant TBTO showed that TBTO affects the kinase p70S6K (the upstream regulator of RPS6) but via a different mechanism than the 
immunosuppressive drug rapamycin, which is a selective inhibitor of mTOR. Finally, the comparison of the transcriptome and protein phosphorylation data of TBTO-treated Jurkat cells revealed both known and novel signaling networks that mediate the cellular response to TBTO.

\section{Materials and Methods}

Chemicals and antibodies. All chemicals tested were purchased from Sigma-Aldrich (Zwijndrecht, The Netherlands). Stock solutions of all chemicals were made by dissolving the substances in dimethyl sulfoxide (DMSO; Merck, Darmstadt, Germany). Primary antibodies against total mTOR (2983), phospho-mTOR S2481 (2974), phospho-mTOR S2448 (2971), total p70S6K (9202), phosphorp70S6K T389 (9205), total RPS6 (3944), phosphor-RPS6 S235/6 (4858), phosphor-RPS6 S240/4 (5364), and fluorescent secondary antibodies anti-mouse IgG (PE conjugated, 8887) and anti-rabbit IgG (Alexa Fluor ${ }^{\circledR} 488$ conjugated, 4412) were purchased from Cell Signaling Technology (Cell Signaling Technology via Bioké, Leiden, The Netherlands). Fluorescent secondary antibodies for western blotting, including IRDye 800CW Donkey anti-Mouse IgG (926-32212), IRDye 800CW Donkey anti-Rabbit IgG (926-32213), IRDye 680RD Donkey anti-Mouse IgG (926-68072), and IRDye 680RD Donkey anti-Rabbit IgG (926-68073), were purchased from LiCor Biotechnology GmbH (Bad Homburg, Germany).

Cell culture. The Jurkat cell line was purchased from the American Type Culture Collection (ATCC $®$ TIB-152 ${ }^{\mathrm{TM}}$, MD). The cells were grown in RPMI-1640 medium supplemented with $10 \%$ heat inactivated fetal calf serum (FCS), $2 \mathrm{mM}$ glutamine, $1 \mathrm{mM}$ sodium pyruvate, $1 \mathrm{mM}$ nonessential amino acids, 100 $\mathrm{U} / \mathrm{ml}$ penicillin, and $100 \mu \mathrm{g} / \mathrm{ml}$ streptomycin (all reagents from Sigma). The cells were cultured T-75 flasks (Gibco, Breda, The Netherlands) in a humidified atmosphere at $37{ }^{\circ} \mathrm{C}$ with $5 \% \mathrm{CO}_{2}$. The culture medium was refreshed every two days.

Chemical exposures. Jurkat cells between passages 16-19 were used in the exposure experiments. Specifically, $2.7 \mathrm{ml}$ medium containing 750,000 cells were seeded in each well of 6-well plates and the cells were allowed to grown for $20 \mathrm{~h}$ before the exposure. Exposure was initiated by adding $0.3 \mathrm{ml}$ medium containing the test compounds (at subcytotoxic concentrations, TBTO 100nM, TBTC 100nM,

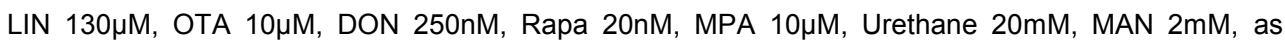
determined after $24 \mathrm{~h}$ exposure in (Shao et al., 2013)) or vehicle controls. The final DMSO concentration in the medium was $0.1 \%(\mathrm{v} / \mathrm{v})$ for all the samples. Exposures were performed on three independent days with Jurkat cells of different passages. 
Cell lysate preparation. After exposure, cells were collected by centrifugation ( 5 min at $300 \mathrm{~g}$ ) and washed with phosphate buffered saline (PBS). Cell pellets were resuspended in $300 \mu \mathrm{l}$ of M-PER mammalian protein extraction reagent (ThermoFisherScientific, Etten-Leur, The Netherlands) supplemented with $1 \%$ protease inhibitor cocktail and $1 \%$ phosphatase inhibitor cocktail (ThermoFisherScientific). The samples were kept on ice for $30 \mathrm{~min}$, and then centrifuged for $30 \mathrm{~min}$ at $10,000 \mathrm{~g}$. The supernatants (lysates) were collected and stored at $-80^{\circ} \mathrm{C}$ until further analysis. Protein concentration was determined by Bio-Rad DC protein assay (Veenendaal, The Netherlands) according to manufacturer's protocol.

Pathscan RTK Signalling antibody array. The Pathscan RTK Signalling antibody array (Cell Signaling Technology, Danvers, MA) is a slide-based antibody array which is based upon the sandwich immunoassay principle. In total, 28 receptor tyrosine kinases and 11 crucial signalling nodes can be detected when they are phosphorylated at tyrosine or indicated residues. This experiment was carried out in biological triplicate at four time points according to the manufacturer's protocol. For each sample, $150 \mu \mathrm{g}$ of cell lysate was loaded on the array.

Western blotting. An aliquot (16 $\mu \mathrm{g}$ protein/sample) of test lysate was loaded onto a $10 \%$ MiniPROTEAN TGX pre-cast gel (Bio-Rad) under reducing conditions. After resolution, the proteins were electrotransferred to a $0.45-\mu \mathrm{m}$ PVDF membrane (Millipore, Amsterdam, the Netherlands). The membrane was then blocked in Odyssey Blocking Buffer (LiCor Biotechnology $\mathrm{GmbH}$ ) for $1 \mathrm{hr}$ at room temperature. All primary antibodies were diluted in 5\% BSA in TBS with 1\% Tween (TBS-T), and then added to the system and the membrane was incubated at $4^{\circ} \mathrm{C}$ overnight. After repeated washes with TBS-T, secondary antibodies diluted in 5\% Protifar (Bio-Rad) in TBS-T, were placed in the system and the membrane was incubated at room temperature for $1 \mathrm{hr}$. Thereafter, the membrane was washed with TBS-T and then scanned using the Odyssey Infrared Imaging System (LiCor Biotechnology $\mathrm{GmbH})$.

Flow cytometry. Exposed cells were collected by centrifugation ( $5 \mathrm{~min}$ at $300 \mathrm{~g}$ ) and washed once with ice cold PBS. The cell pellet was then resuspended in $500 \mu \mathrm{l}$ fresh ice cold PBS. And $4.5 \mathrm{ml}$ of methanol was added for fixation and permeabilization. After $30 \mathrm{~min}$ incubation on ice, the cells were pelleted and washed two times with ice cold PBS. The cell pellet was then resuspended in PBS with $10 \%$ FCS (incubation buffer), and aliquoted into eppendorf tubes. Primary antibodies against total RPS6 (CST2317), phospho-RPS6 S235/6 (CST4858), phospho-RPS6 S240/4 (CST5364) were added to separate samples at dilutions provided by the manufacturers. After incubation at room temperature for $1 \mathrm{~h}$, all the samples were centrifuged. The resulting cell pellets were washed once with ice cold PBS and then resuspend in fresh incubation buffer. Secondary antibodies (anti-mouse IgG, PE conjugated, CST8887 for total RPS 6 and anti-rabbit IgG, Alexa Fluor ${ }^{\circledR} 488$ conjugated, CST 4412 for 
phosphor-RPS6 S235/6 and phosphor-RPS6 S240/4) were added to the corresponding samples according to their dilution factors provided by the manufacturers. After incubation for $30 \mathrm{~min}$ at room temperature, stained cells were pelleted, washed once with incubation buffer, and resuspended in PBS. All the samples were then examined by flow cytometry using a Cyan ADP Analyzer (Beckman Coulter, Woerden, The Netherlands) and Summit software 4.3 (Beckman Coulter). A minimum of 5,000 events per sample was acquired.

Gene set enrichment analysis. Gene set enrichment analysis (GSEA) was conducted as described before (Shao et al., 2013). The transcriptome dataset has been published online (GSE46909). Gene sets were used from a total of eight different collections, i.e., Gene Ontology (GO) (http://www.geneontology.org/) including biological process, cellular component, and molecular function, Biocarta-2 (http://www.biocarta.com), KEGG (http://www.genome.jp/kegg), Reactome (http://www.reactome.org), Tox-action (self-made), and Lymphocyte database (Shaffer et al., 2001). Gene sets with a $p$ value $<0.01$ and an FDR value $<0.25$ were considered as significantly regulated.

Molecular concept analysis. Molecular concept analysis allows visualization of the network in which the overlap between gene sets based on co-occurrence of genes is shown. This overlap was calculated based on the genes that were responsible for a gene set to be considered as significantly affected. For this, either the top $20 \%$ upregulated genes or the top $20 \%$ downregulated genes were used. To reduce the complexity of the figures, the selection criteria for significant gene sets were set at $p$ value $<0.01$ and an FDR value $<0.1$. In addition, gene sets containing $\leq 10$ genes were excluded from the analysis. The significance of overlap between gene sets was calculated based on the binomial distribution using Venn-Mapper (Smid et al., 2003). Gene sets showing significant overlap (a $z$ value $>5.2$ equals to a $p$ value $<0.0000001$ ) were connected in a network which was visualized using Cytoscape (Shannon et al., 2003). Gene sets with high overlap (high $z$ values) were clustered close to each other, and are expected to share biological processes.

Chemotaxis assay. Chemotaxis assay was performed according to the Boyden chamber assay (Chen, 2005), with slight modifications. Jurkat cells (passage number 16-19) were seeded in $900 \mu \mathrm{l}$ medium per well in regular 12-well plates (60,000 cells/well). After growing the cells for $20 \mathrm{~h}$, exposure was initiated by adding $100 \mu \mathrm{l}$ medium containing either $1 \mu \mathrm{m}$ TBTO (final exposure concentration: $100 \mathrm{nM}$ ) or vehicle controls. Cells were exposed to the compounds for $22 \mathrm{~h}$. The final DMSO concentration in the medium was $0.1 \%(\mathrm{v} / \mathrm{v})$ for all samples. After exposure, cells were counted and transferred to apical compartments of trans-well chemotaxis chambers $(150 \mu \mathrm{l} / \mathrm{insert})$. The pore size of the insert was $5 \mu \mathrm{m}$ (Corning Life Science B.V., Amsterdam, The Netherlands). A total of $500 \mu \mathrm{l}$ of medium containing $25 \mathrm{ng} / \mathrm{ml}$ of $\mathrm{CXCL} 12$ (Biosource, Camarillo, CA) and the same concentration of either TBTO $(100 \mathrm{nM})$ or DMSO $(0.1 \%)$ were added in the basolateral compartments. The migration was 
then allowed to occur for $2 \mathrm{~h}$ at $37^{\circ} \mathrm{C}$. After this period, the cells that migrated into the basolateral compartment were counted by Coulter Counter (Z2 Coulter Particle Count and Size Analyzer, Beckman Coulter Netherland BV, Woerden, The Netherlands). The percentage of the migrated cells were then calculated and compared to the results with wells that contained only DMSO.

\section{Results}

Identification of cellular signaling pathways affected by direct immunotoxicants

In order to identify the signaling pathways that are commonly regulated by immunotoxicants, timedependent effects of seven immunosuppressants, including five immunotoxicants (lindane, ochratoxin A, TBTC, TBTO, and DON), and two immunosuppressive drugs (rapamycin and mycophenolic acid), as well as two nonimmunotoxic control chemicals (urethane and mannitol), were assessed upon exposure of Jurkat cells to subcytotoxic concentrations. Upon treatment, cells were lysed and subjected to protein phosphorylation profiling using PathScan RTK Signalling antibody arrays. Hierarchical clustering analysis revealed that the following cellular signaling events were affected by at least three immunotoxicants (Fig. 1). The most predominant effects were observed on the phosphorylation of RPS6 (Ser235/6), which was increased by DON ( $\geq 1.84$-fold, from $3 \mathrm{~h}$ onwards) and decreased by rapamycin ( $\leq-1.99$-fold, from $0.5 \mathrm{~h}$ onwards), lindane $(\leq-1.97$-fold, from $0.5 \mathrm{~h}$ onwards), TBTO ( $\leq-2.99$-fold, from $3 \mathrm{~h}$ onwards), ochratoxin A (-2.14-fold, at $6 \mathrm{~h}$ ), and mycophenolic acid (-1.80-fold, at $24 \mathrm{~h}$ ) (Supplementary fig. 1). Phosphorylation of Akt at Thr308 position was increased by lindane, TBTO, and mycophenolic acid ( $\geq 1.33$-fold, from $0.5 \mathrm{~h}$ onwards) and slightly increased by rapamycin at earlier time points ( $\geq 1.22$-fold, from 0.5 till $6 \mathrm{~h}$ ). Another phosphorylation position of Akt at Ser473 was increased by lindane ( $\geq 1.30$-fold, from $0.5 \mathrm{~h}$ onwards), mycophenolic acid ( $\geq 1.28$-fold, from $0.5 \mathrm{~h}$ onwards), and TBTC ( $\geq 1.57$-fold, from $3 \mathrm{~h}$ onwards) but decreased by ochratoxin A (-1.64-fold, at $6 \mathrm{~h}$ ) and rapamycin ( $\leq-1.8$ - fold, from $3 \mathrm{~h}$ onwards). The phosphorylation of Src at pan-Tyr position was found to be increased by lindane ( $\geq 1.55$-fold, from $3 \mathrm{~h}$ onwards), ochratoxin A ( $\geq 1.79$-fold, from $6 \mathrm{~h}$ onwards), TBTC (1.69-fold, at $24 \mathrm{~h})$, TBTO (1.68-fold, at $24 \mathrm{~h}$ ), and rapamycin ( $\geq 1.50$-fold, from $0.5 \mathrm{~h}$ onwards) but decreased by TBTO $(-1.27$-fold, at $3 \mathrm{~h}$ ) and DON $(\leq-$ 1.53-fold, from $0.5 \mathrm{~h}$ onwards). The phosphorylation of $\mathrm{p} 44 / 42$ at Thr202/Tyr204 position was increased by lindane at earlier time points ( $\geq 1.58$-fold, from 0.5 till $6 \mathrm{~h}$ ) and rapamycin (1.38-fold, at 3 h) but decreased by lindane $(-2.18$-fold, at $24 \mathrm{~h}$ ), ochratoxin $A(\leq-1.24$-fold, from $0.5 \mathrm{~h}$ onwards), TBTC (-2.41-fold, at $24 \mathrm{~h}$ ), TBTO ( $\leq-1.22$-fold, from $3 \mathrm{~h}$ onwards), and mycophenolic acid (-1.49-fold, at $6 \mathrm{~h}$ ). The phosphorylation of Lck at pan-Tyr position was found to be increased by TBTC (1.92-fold, at $24 \mathrm{~h}), \mathrm{DON}$ (1.90-fold, at $24 \mathrm{~h})$, and mycophenolic acid (1.90-fold, at $24 \mathrm{~h}$ ) but decreased by lindane ( $\leq-2.97$-fold, from $6 \mathrm{~h}$ onwards), ochratoxin A (-1.65-fold, at $24 \mathrm{~h})$, and by DON at earlier time points ( $\leq-1.53$-fold, from 0.5 till $3 \mathrm{~h}$ ). However, this kinase was also affected from $3 \mathrm{~h}$ onwards by the two nonimmunotoxic control chemicals urethane ( $\geq 1.32$-fold) and mannitol ( $\geq 1.28$-fold). 
In addition, the following RTKs/ signaling nodes were found to be affected by two immunotoxicants (Fig. 1). The phosphorylation of STAT3 at Tyr705 position was increased by TBTO ( $\geq 1.31$-fold, from $0.5 \mathrm{~h}$ onwards) and decreased by lindane ( $\leq-1.30$-fold, from $3 \mathrm{~h}$ onwards). The phosphorylation of EphB1 and EphB3 at pan-Tyr position was found to be slightly increased by TBTO and DON ( $\geq 1.21$ fold, from $0.5 \mathrm{~h}$ onwards).

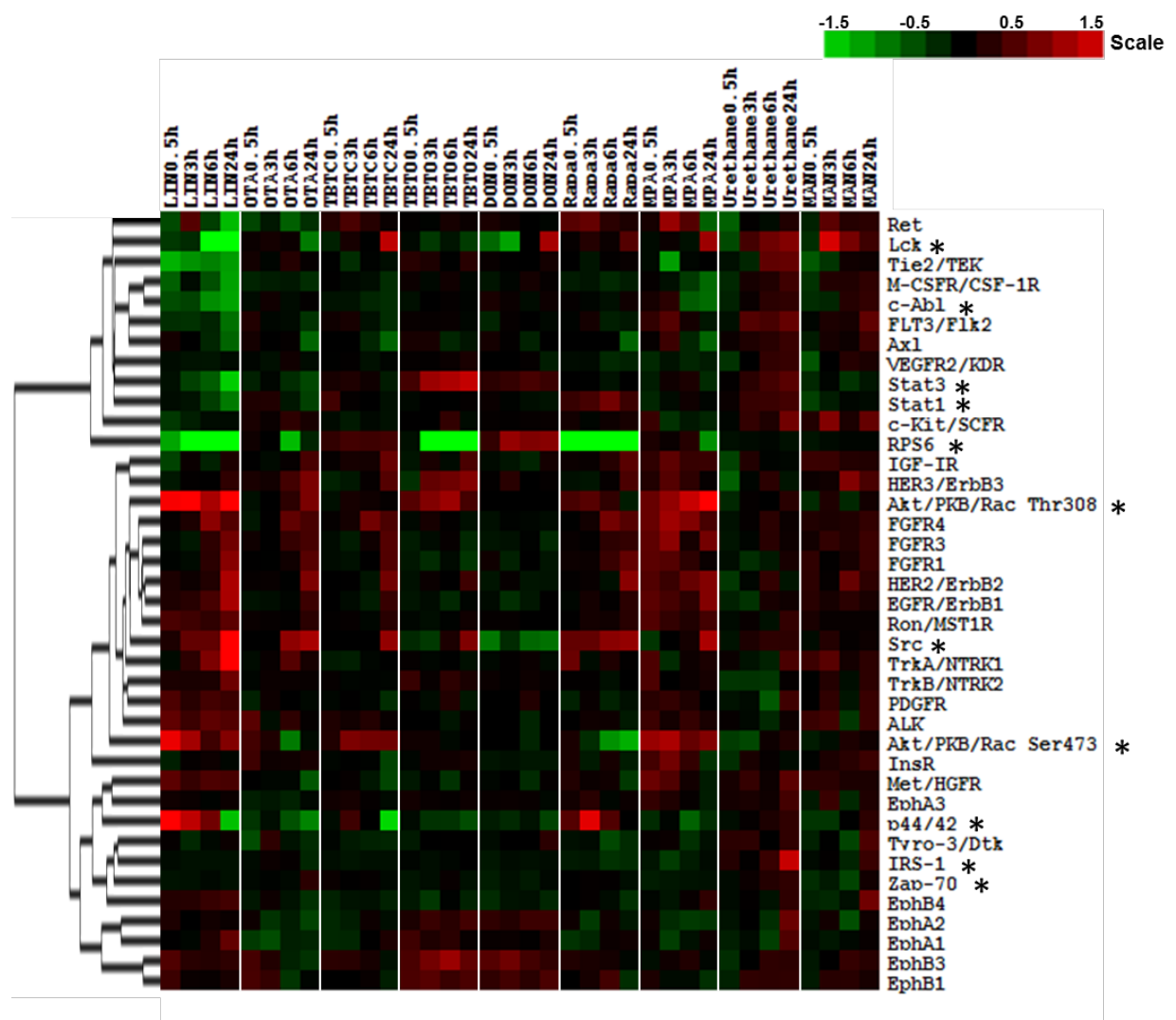

FIG. 1. Unsupervised hierarchical clustering of the phosphorylation of 28 receptor tyrosine kinases and 11 important signaling nodes. The heat map visualizes the phosphorylation of 28 receptor tyrosine kinases and 11 important signaling nodes (with *) in Jurkat cells upon exposure to one of five immunotoxicants (i.e. lindane, ochratoxin A, TBTC, TBTO, and DON), or two immunosuppressive drugs (i.e. rapamycin and mycophenolic acid), and two nonimmunotoxicants (i.e. urethane and mannitol) for 0.5, 3, 6, and $24 \mathrm{~h}$. Green represents a decrease in phosphorylation, red an increase in phosphorylation, and black no regulation, as compared to the carrier control of each exposure time point. The color intensity related to the $2 \log$ ratio is indicated by the bar.

TBTO-mediated effects on phosphorylation of RPS6 and other mTOR pathway intermediates 
To validate the PathScan array data and further expand from one kinase/ signaling node to a whole signalling pathway, we selected TBTO as the model immunotoxicant and started validating its effects on the phosphorylation of RPS6, one of the most commonly and clearly affected proteins by immunotoxicants in this study. Flow cytometry experiments on this protein were performed first to confirm the results from the PathScan array experiment (Fig. 2A and 2B), to examine whether this TBTO-mediated dephosphorylation of RPS6 was specific for Ser235/6 position, and to determine whether the reduced levels of phosphorylated RPS6 was due to dephosphorylation or to a decrease of total RPS6 protein. The results revealed that TBTO exposure decreased phosphorylation of RPS6 (Ser235/6) without altering the expression level of total RPS6 (Fig. 2C and 2D). Furthermore, our results demonstrated that this TBTO-mediated dephosphorylation of RPS6 also happened at Ser240/4 position (3.3-fold, after $24 \mathrm{~h}$ ), and to the same extent as at the Ser235/6 position (Fig. 2C and 2D).

A.

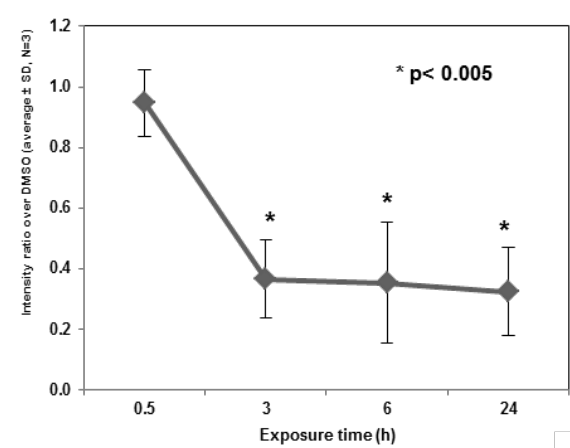

B.

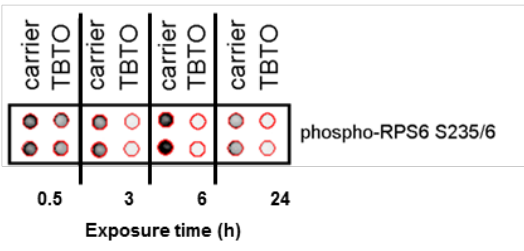

c.

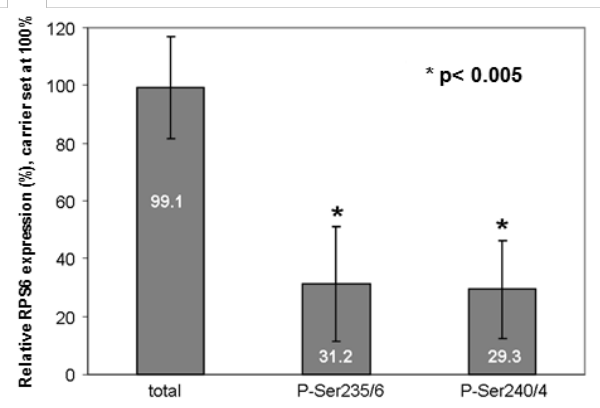

D.

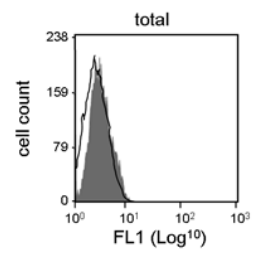

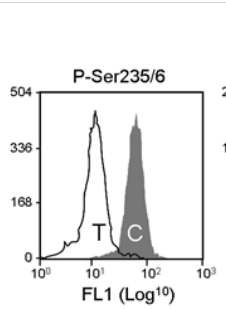

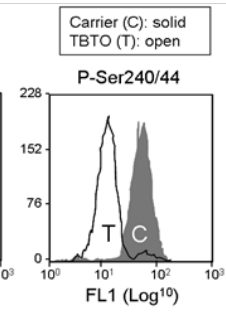

FIG. 2. Effects of TBTO on phosphorylation of RPS6 in time. Jurkat T cells were exposed to 100 nM TBTO for $0.5,3,6$, and $24 \mathrm{~h}$. The average results of the expression levels of phosphorylated RPS6 (Ser235/6) from three independent experiments with RTK antibody array are shown in A, and a representative scan of the RTK antibody array is shown in B. The effects of 24 h TBTO exposure on the expression levels of RPS6 (total, p-Ser235/6 and p-Ser240/4) were further tested by flow cytometry. Average results of three independent experiments were shown in $C$ and representative histograms were shown in $D$. *: $p<0.005$, two sample equal variance $T$ test, two tailed.

RPS6 is the downstream effector of p70 ribosomal protein S6 kinase (p70S6K) and the phosphorylation of the latter kinase is in turn directly controlled by mammalian target of rapamycin (mTOR) in mammalian cells (Chung et al., 1992). Therefore, this study also assessed the effect of TBTO on the phosphorylation of mTOR and p70S6K. Western blotting analysis showed that TBTO 
dephosphorylated p70S6K at Thr389 position starting from $0.5 \mathrm{~h}$ onwards, without affecting the expression levels of total p70S6K (Fig. 3). However, the expression levels of total and phosphorylated mTOR were not changed upon TBTO exposure (Fig. 3).

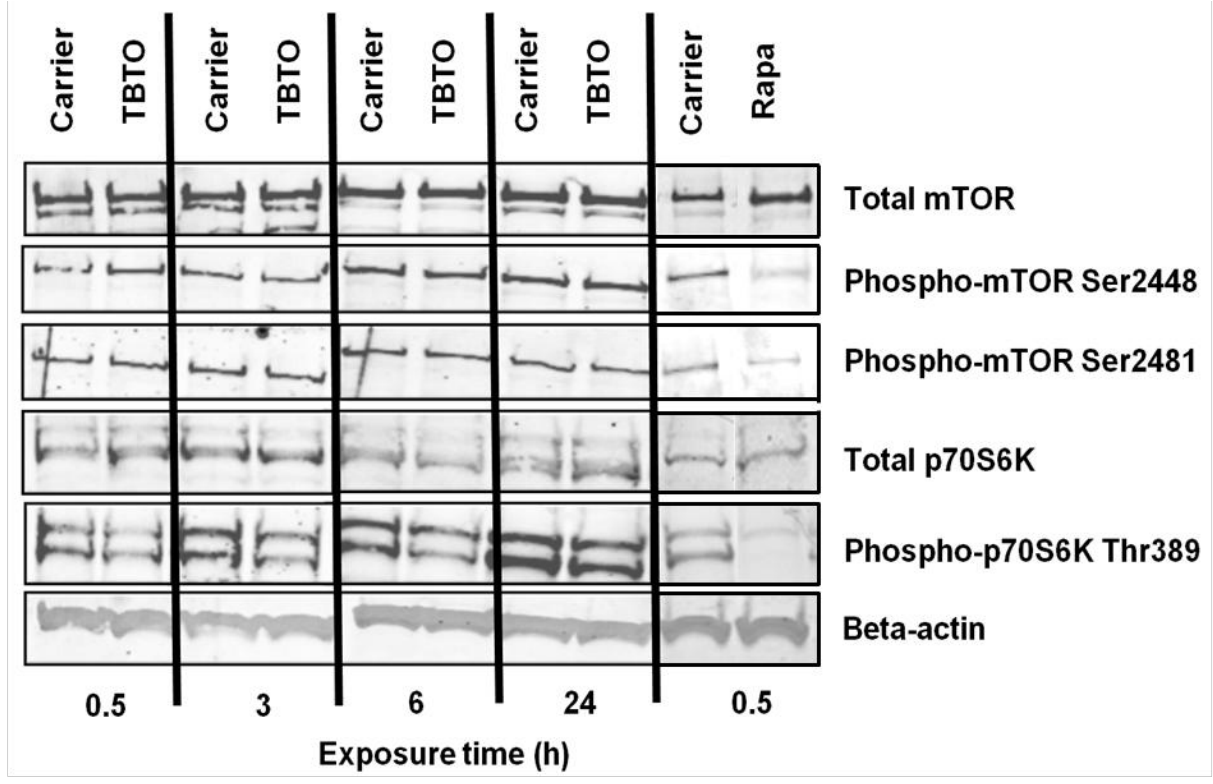

\begin{tabular}{c|c|c|c|c|c|c}
\hline \multirow{2}{*}{ Protein } & \multirow{2}{*}{$\begin{array}{c}\text { Phosphorylation } \\
\text { site }\end{array}$} & \multicolumn{5}{|c}{ Relative ratio (phospho/ total, DMSO set at 100\%) } \\
\cline { 3 - 7 } & & TBTO 0.5h & TBTO 3h & TBTO 6h & TBTO 24h & Rapamycin 0.5h \\
\cline { 3 - 7 } & Ser2448 & 104.8 & 103.22 & 101.5 & 105.9 & 48.7 \\
mTOR & Ser2481 & 102.6 & 96.7 & 101.6 & 102.0 & 46.7 \\
& Thr389 & 78.6 & 75.5 & 66.6 & 77.9 & 34.2 \\
\hline
\end{tabular}

FIG 3. Effects of TBTO on the protein expression levels of mTOR signaling intermediates. Jurkat cells were exposed to $100 \mathrm{nM}$ TBTO for 0.5, 3, 6, and $24 \mathrm{~h}$. The protein expression levels of the following mTOR signaling intermediates were quantified by western blot: total and phosphorylated (Ser2448 and Ser2481) mTOR, and total and phosphorylated (Thr389) p70 ribosomal protein S6 kinase (p70S6K). Quantification of the bands was shown in the table below the picture. The ratios between phosphorylated and total proteins were calculated, and relative ratios were calculated by setting the ratio of the DMSO sample of the corresponding time point at $100 \%$. Rapamycin (20 nM) was used as positive control.

Thus, TBTO exposure caused a decrease in the expression levels of phosphorylated p70S6K at Thr389 position and phosphorylated RPS6 at both Ser235/6 and Ser240/4 positions, without affecting the expression levels of total p70S6K and RPS6. However, in contrast to rapamycin, TBTO did not affect the expression levels of total and phosphorylated mTOR at either Ser2448 or Ser2481 position. 


\section{GSEA analysis of the microarray data of TBTO}

Gene set enrichment analysis (GSEA) of previously generated Jurkat transcriptome data (Shao et al., 2013) was performed in order to identify the gene sets affected by TBTO. Eight different collections of gene sets were used in the analysis (see Materials and Methods). In total, 95 gene sets were significantly affected by TBTO, of which 54 were upregulated and 41 downregulated. Molecular concept mapping on the most significantly affected gene sets was then performed for visualizing the overlap in genes among the significant gene sets (Fig. 4). The results of the molecular concept mapping are shown in Fig. 4. The gene sets upregulated after $6 \mathrm{~h}$ exposure to $100 \mathrm{nM}$ of TBTO can be clustered in three groups: regulation of cell cycle, leukocyte migration, and phosphatidylinositol signaling. The gene sets downregulated after $6 \mathrm{~h}$ exposure to $100 \mathrm{nM}$ of TBTO can be placed in four clusters: ribosome biogenesis, nucleus, biosynthesis of $\mathrm{N}$-glycan, and mitochondrial function. In the last cluster three themes can be further distinguished: mitochondrion ribosome, mitochondrion inner membrane, and mitochondrion electron transport.

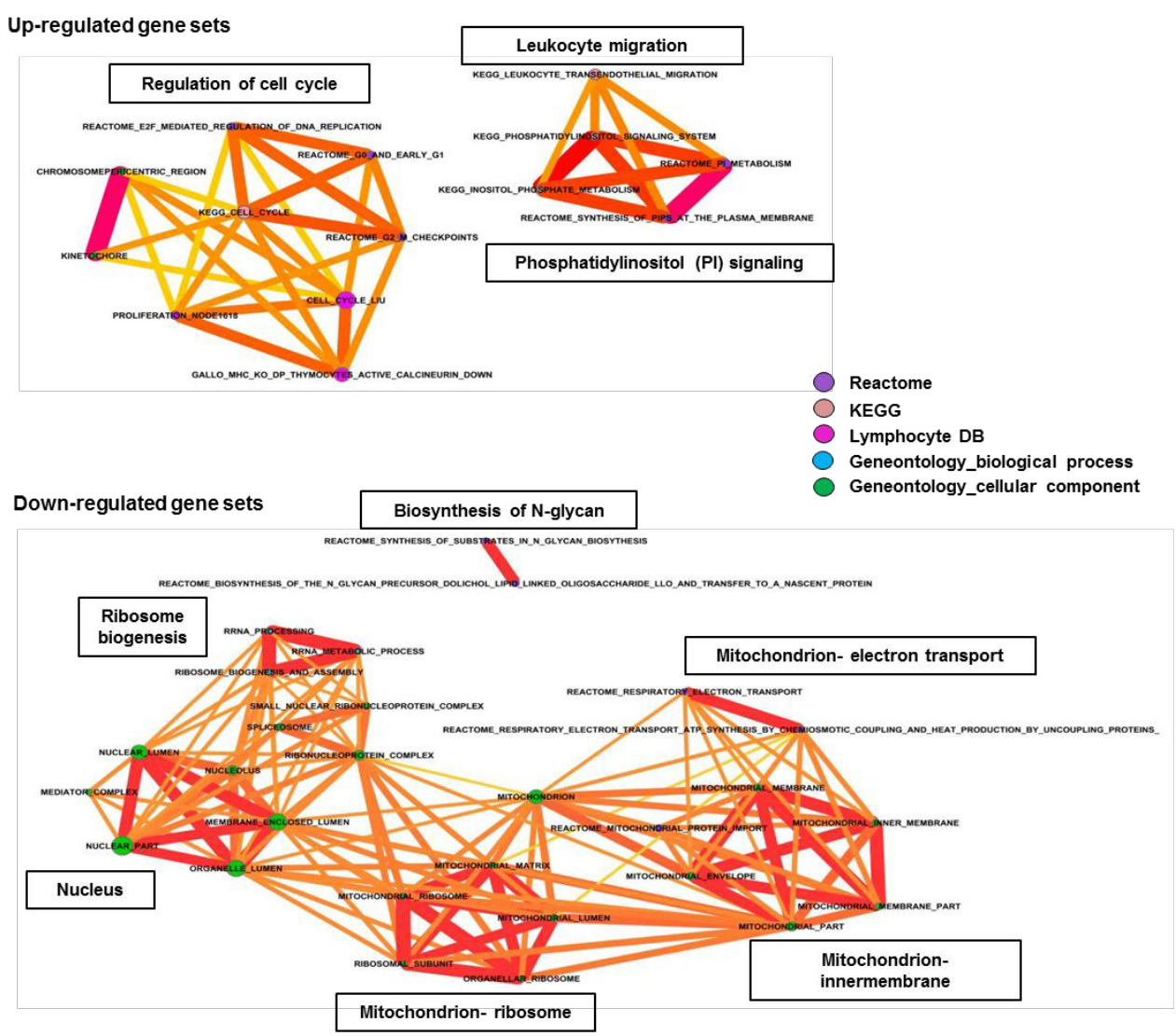


FIG 4. Molecular concept analysis of gene sets affected in Jurkat cells upon 6 h exposure to 100 nM TBTO. For clarity, gene sets with lower significance in overlap or contain $\leq 10$ genes were left out. The size of the nodes corresponds to the number of genes of the gene set. The thickness of the connecting lines indicates the degree of overlap between the gene sets. The colour of the nodes corresponds to the gene set collection from which the gene sets were derived: purple: Reactome; lightsalmon: KEGG; pink: Lymphocyte DB; blue: Geneontology_biological process; green: Geneontology_cellular component.

Regarding the upregulated biological processes, GSEA analysis clearly indicates that TBTO upregulated genes involved in the cell cycle related processes including DNA replication, mitosis, and cell division (PRIM2, BUB1, and CDK6) (Fig. 4). TBTO, however, also induced genes that are known to be involved in cell cycle arrest and apoptosis (SP1, CASP2, and CLIC4). The expression levels of these genes are shown in Supplementary fig. 2A. Another biological process that was upregulated by TBTO is leukocyte migration (Fig. 4). As shown in Fig. 5A, many genes that are known to be essential for leukocyte migration were upregulated by TBTO treatment, including ROCK1, ROCK2, and several leukocyte integrins, i.e., ITGA4, ITGB2 (alias CD18), and ITGAL (alias CD11a). Several gene sets related with phosphatidylinositol signaling were also up-regulated by TBTO treatment (Fig. 4); these included genes encoding different phosphorylated forms or subunits of PI3-kinase (PI3K) and genes encoding two members of the inositol-polyphosphate 5-phosphatase (PIPP) family: SYNJ1 (alias INPP5G) and SYNJ2 (alias INPP5H), which are regulators of the PI3K signaling pathway (Fig. 5B).

Biological processes downregulated by TBTO included gene sets associated with ribosome biogenesis (Fig. 4). These gene sets include genes encoding several factors that are known to be involved in biogenesis and quality control of ribosomes, i.e., RRP9, NOP58, NOP56, RPP30, RPP38, RPP40, EXOSC2, EXOSC3, and EXOSC7 (Fig. 5C). In addition, TBTO downregulated mitochondrial ribosome RNA levels (Fig. 5C, Supplementary fig. 2C). TBTO also downregulated mRNAs of genes involved in the inner membrane and electron transport of mitochondria including a number of TIMMs (e.g. TIMM9, 22, 23, and 50) and NDUFs (e.g. NDUFS4, S8, A5, and A6), which encoding translocases of the inner membrane and NADH dehydrogenases, respectively (Supplementary fig. 2D and 2E) (Fig. 4). In addition, TBTO downregulated gene sets related to the nucleus (Fig. 4). Part of the genes involved in this cluster (i.e., RRP9 and various RPPs, EXOSCs, and MRPs) overlapped with the genes involved in ribosome biogenesis and mitochondrial ribosomes (Fig. 4, Supplementary fig. 2B). In addition, these gene sets also involved genes encoding pre-mRNA splicing proteins, including SF3B3, SF3A2, PRPF18, and PRPF31 (Supplementary fig. 2B). Therefore, based on the genes of this cluster, it is clear that TBTO inhibited transcription and translation processes. Two gene sets related with $\mathrm{N}$-glycan biosynthesis were found to be down-regulated by TBTO (Fig. 4). These gene sets include genes encoding glycosyltransferases (ALG1, 3, 5, 12, and 14) and dolichyl-phosphate mannosyltransferase polypeptide 2 (DPM2) (Supplementary fig. 2C).

Part of the pathways that are revealed by GSEA analysis are also indicated by the protein phosphorylation data of Jurkat cells in response to TBTO. For instance, the dephosphorylation of 
RPS6 indicated an inhibitory effect of TBTO on ribosome biogenesis, since RPS6 is a crucial component involved in this process (Meyuhas, 2000; Salmond et al., 2009a). TBTO also affected the phosphorylation of Stat3, and slightly induced the phosphorylation of EphB1 and EphB3 (Fig. 1). The JAK/STAT pathway has been reported to be activated in CXCL12/ CXCR4 mediated chemotaxis in several human T cell lines (Vila-Coro et al., 1999), whereas the activation of ephrin receptors have been shown to inhibit this process in a human T cell line and primary T cells (Sharfe et al., 2002).

Taken together, both transcriptome and protein phosphorylation data of Jurkat cells indicated that TBTO affected ribosome biogenesis and cell migration.

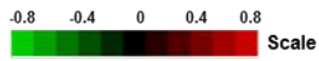

A. Leukocyte migration

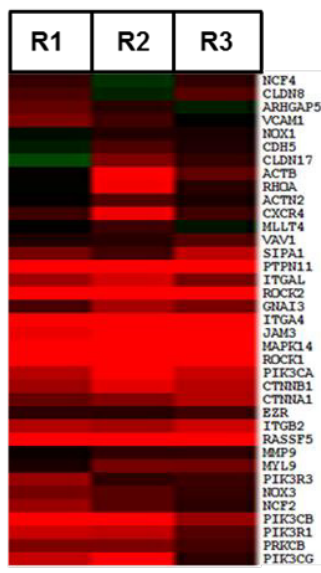

B. PI signaling

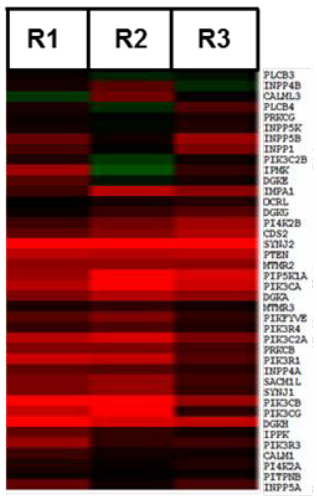

\section{Ribosome biogenesis}

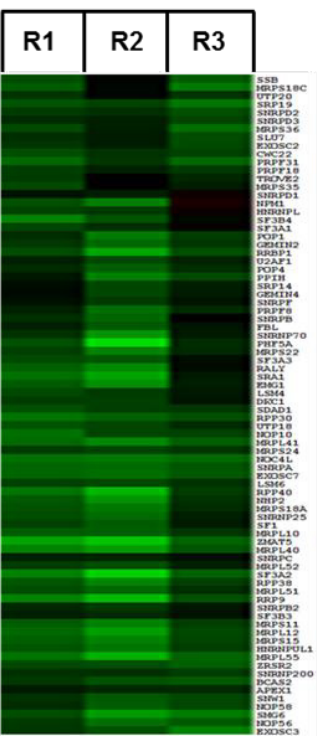

FIG. 5. Effects of TBTO on the expression levels of genes involved in leukocyte migration, phosphatidylinositol (PI) signaling, and ribosome biogenesis. The heat maps visualize the effects of TBTO exposure on the expression levels of genes involved in three different biological processes in Jurkat cells. Upon exposure for $6 h$, TBTO upregulated genes that are involved in leukocyte migration (A) and phosphatidylinositol signaling (B), and downregulated the genes that are involved in ribosome biogenesis (C). The heat maps show $2 \log$ ratios of TBTO vs. average of DMSO controls (in three biological replicates, $R 1$ to $R 3$ ). Green represents downregulation, red upregulation, and black no regulation. The color intensity is related to the $2 \log$ ratio and is indicated by the bar. 


\section{TBTO inhibited chemotaxis of Jurkat cells towards the chemokine CXCL12}

A trans-well based chemotaxis assay using the chemokine CXCL12 was performed in order to confirm the hypothesis that TBTO would affect the chemotaxis process of Jurkat cells, which was indicated by both transcriptome and protein phosphorylation data. The results showed that CXCL12 is a potent chemoattractant, as the number of migrated Jurkat cells increased significantly when CXCL12 was added in the basolateral compartment of the trans-well system (Fig. 6). In addition, the treatment of TBTO (100 nM, 24 h) significantly decreased the number of Jurkat cell migrated towards CXCL12.

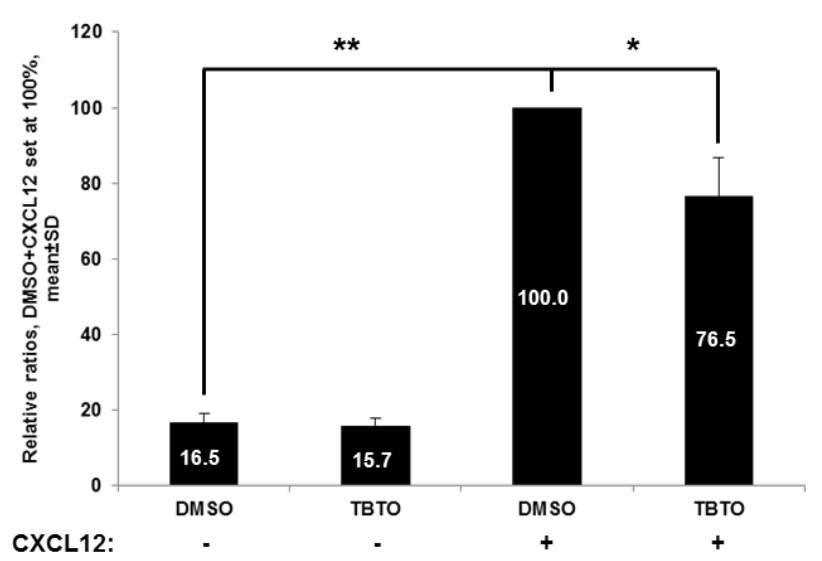

FIG. 6. Effects of TBTO on the chemotaxis of Jurkat cells towards the chemokine CXCL12. The bar graph shows the effect of TBTO treatment (100 nM) on the chemotaxis of Jurkat cell towards the chemokine CXCL12. CXCL12 is shown as a potent chemoattractant, as the amount of migrated Jurkat cell increased significantly when CXCL12 is added in the basolateral compartment. Upon exposure for $24 \mathrm{~h}$, TBTO decreased the percentage of migrated Jurkat cells to $76.5 \%$. *: $p<0.05$; **: $p<0.01$, two sample equal variance $T$ test, two tailed.

\section{Discussion}

The objective of the present study was to identify the cellular signaling pathways that are possibly involved in chemical-induced direct immunotoxicity. To that end, we used an antibody array to assess the effects of five immunotoxicants, two immunosuppressive drugs, and two nonimmunotoxic control chemicals on protein phosphorylation in human Jurkat T-cells in vitro. We identified in total four kinases/ proteins, i.e., RPS6, Akt, Src, and p44/42, whose phosphorylation was affected by at least three immunotoxicants and not by the nonimmunotoxic control chemicals. The most predominant effect was the modulation of the phosphorylation of RPS6. Further validation experiments with TBTO and rapamycin suggested that although a number of immunotoxicants modulated mTOR pathway, the underlying mechanisms were chemical-dependent. We also compared the transcriptome to the protein 
phosphorylation profiling data of TBTO. Both data indicated that TBTO affects ribosome biogenesis and leukocyte migration in Jurkat cells.

The phosphorylation of the kinase Akt, at either Thr308 or Ser473 position, was shown to be affected by a number of immunotoxicants (Fig. 1). Lindane and mycophenolic acid induced phosphorylation of Akt at both positions (Fig. 1). Akt plays an essential role in multiple fundamental cellular processes such as transcription, protein synthesis, cell proliferation, and apoptosis (Fayard et al., 2005). With respect to the immune system, a growing body of evidence now exists to show that Akt activation is associated with various immune-related pathologies including leukemia and autoimmune diseases (Bellacosa et al., 2005; Min et al., 2003; Patel and Mohan, 2005). Phosphorylation at the Thr308 position of Akt is known to be regulated through the PI3K/ PDK1 pathway (Alessi et al., 1997), whereas phosphorylation at the Ser473 position is thought regulated by mTORC2 (Sarbassov et al., 2005). Full activation of Akt requires phosphorylation at both positions (Hay and Sonenberg, 2004). Activated Akt will then activate mTORC1 via direct phosphorylation and inhibition of proline-rich Akt substrate of $40 \mathrm{kDa}$ (PRAS40) and tuberous sclerosis complex 2 (TSC2) (Brazil and Hemmings, 2001; Haar et al., 2007). In addition, partial activation of Akt by phosphorylation at Thr308 position alone has been demonstrated to be sufficient to activate mTORC1 (Haar et al., 2007).

The direct substrates of mTORC1 include p70S6K and eukaryotic translation initiation factor 4E binding protein 1 (4EBP1) (Hemmings and Restuccia, 2012). The activation of p70S6K by mTORC1 will in turn phosphorylate RPS6. Therefore, since Akt and RPS6 are in the same signaling pathway, it can be expected that the compounds that target this pathway by modulating Akt activity will affect the phosphorylation of RPS6 in the same direction. However, our results in Jurkat cells showed that the immunotoxicants lindane and TBTO induced the phosphorylation of Akt at Thr308 position throughout the duration of treatment, but dephosphorylated RPS6 (lindane from $0.5 \mathrm{~h}$ onwards, TBTO from $3 \mathrm{~h}$ onwards) (Fig. 1). These results indicated that the decreased phosphorylation of RPS6 induced by these two agents was independent from their modulation of Akt activity. Due to the early alteration in RPS6 activity in response to the exposure to these two compounds, it can be expected that these two compounds also target a protein/kinase located in the mTORC1-p70S6K pathway. Similar results have been reported before, as Chlamydia (Chlamydophila) pneumoniae infection activated Akt at both Thr308 and Ser473 positions in Jurkat cells, whereas the phosphorylation of p70S6K was inhibited (Hirai et al., 2013).

In the present study, phosphorylation of Src was induced by lindane, ochratoxin A, and rapamycin, and suppressed by DON (Fig. 1). Src belongs to the Src family tyrosine kinases which includes nine non-receptor tyrosine kinases that regulate cellular metabolism, survival, and proliferation (Thomas and Brugge, 1997). Src family kinase inhibitors like Bosutinib and Dasatinib, have been developed as candidate therapeutic agents for several malignancies, including solid tumors and melanoma, and have been approved by USFDA as treatment for leukaemia (Araujo and Logothetis, 2010; Cortes et al., 2012; Homsi et al., 2009; Zwaan et al., 2013). Regarding the immune system, Src family kinases are known to initiate and amplify numerous intracellular signaling pathways that are involved in innate 
and adaptive immune responses, including cell cycle, cell differentiation, migration, adhesion, and phagocytosis (Byeon et al., 2012; Korade-Mirnics and Corey, 2000; Salmond et al., 2009b). In various in vitro cell systems, Src kinase has been demonstrated to phosphorylate p44/42 via the activation of Raf-1 and MEK1/2 (Bełtowski et al., 2006; Guo et al., 2006; Hu et al., 2009; Shan et al., 2001). Our data showed that the activity of $\mathrm{Src}$ and $\mathrm{p} 44 / 42$ were regulated towards the same direction by lindane and rapamycin, suggesting these two immunosuppressants activated $p 44 / 42$ via activation of Src (Fig. 1). These results are in agreement with the findings that both lindane and rapamycin activated p44/42 in a Src-dependent manner in vitro, as pre-treatment with PP2 (a selective Src inhibitor) completely abrogated the activation of p44/42 (Chaturvedi et al., 2009; Mograbi et al., 2003).

Besides the modulation on phosphorylation of Akt, Src, and p44/42 by the various test immunotoxicants, the most predominant effect on protein phosporylation was found for RPS6 (Fig. 1). In total six immunotoxicants were found to modulate RPS6 phosphorylation, of which the mycotoxin DON induced phosphorylation of RPS6, whereas the other five (lindane, ochratoxin A, TBTO, rapamycin, and mycophenolic acid) suppressed the phosphorylation. RPS6 has been demonstrated to be an essential determinant in ribosome biogenesis (Meyuhas, 2000; Salmond et al., 2009a). Deletion of the RPS6 gene in mice resulted in a failure to form functional ribosomes, which eventually led to embryonic lethality; deletion of RPS6 in T cells abrogated T cell development (Šulić et al., 2005). Moreover, those Authors also showed that hemizygous expression of RPS6 impaired T cell proliferation in response to TCR stimulation via activation of a p53-dependent cell cycle checkpoint (Šulić et al., 2005). The phosphorylation of RPS6 is suggested to be controlled by the activities of p70S6K and p90S6K (RSK), in which p70S6K is generally believed to play a predominant role (Meyuhas and Kwang, 2008). The activation of RSK is under the control of p44/42 (Roux et al., 2007). Since none of the compounds that affected the phosphorylation of RPS 6 had prolonged effects on the phosphorylation of p44/42, the contribution of RSK in the modulation of RPS 6 phosphorylation by the tested compounds might be limited.

Rapamycin is a specific inhibitor of the mTOR pathway; therefore, the rapid and drastic dephosphorylation of RPS6 in Jurkat cells in response to rapamycin treatment was expected. Similar effects have also been reported for rapamycin in various cell lines and mouse primary cells in vitro (Chung et al., 1992; Holz and Blenis, 2005; Pende et al., 2004) and in rodents in vivo (Khalaileh et al., 2013). Treatment with other immunotoxicants, lindane, DON and TBTO, also induced an early effect on RPS6 phosphorylation starting from $0.5 \mathrm{~h}$ (for lindane) or $3 \mathrm{~h}$ (for DON and TBTO) of exposure and throughout the whole treatment period. These results indicated that RPS6 (or other mTORC1 pathway intermediates) might be one of the primary targets of these three immunotoxicants. The immunosuppressive drug mycophenolic acid also dephosphorylated RPS6 but only after $24 \mathrm{~h}$ of exposure; this implied this outcome might be an indirect effect of mycophenolic acid. The finding that DON had the opposite effect on the phosphorylation of RPS6 as compared to TBTO was in line with the results obtained from previous transcriptome experiment with Jurkat cells, in which DON upregulated the gene sets related with ribosome biogenesis and pre-mRNA splicing (See Supplementary fig. 3B in (Shao et al., 2013), whereas TBTO downregulated these gene sets (Fig. 4). 
Further investigation on the mTORC1 pathway using the model immunotoxicant TBTO revealed that TBTO, just like immunosuppressive rapamycin, suppressed the phosphorylation of RPS6 at both Ser235/6 and Ser240/4 positions (Fig. 2), and the upstream regulator p70S6K at Thr389 position (Fig. 3). However, a crucial difference between the two compounds is that rapamycin directly targets on mTOR, the upstream regulator of p70S6K, whereas TBTO had no effect on the phosphorylation of mTOR (Fig. 3). This observation indicated that the molecular mechanisms behind this inhibition are different between rapamycin and TBTO, although both compounds inhibited the p70S6K pathway. Our results are in line with the previously reported findings by Osman and van Loveren, 2012, that TBTO down-regulated the expression level of phosphorylated p70S6K in the mouse thymoma cell line EL4 (Osman and van Loveren, 2012). In addition, recent results from Osman and van Loveren have shown that although TBTO dephosphorylated p70S6K, the phosphorylation of the other downstream effector of mTORC1, 4EBP1, was induced by TBTO, whereas rapamycin dephosphorylated this protein (Osman and van Loveren, 2013). This result also indicated that the dephosphorylation of p70S6K by TBTO was independent of mTOR. Further investigation would be interesting in order to identify the targets of other immunotoxicants that also affected the phosphorylation of RPS6 in the present study.

Activation of p70S6K has also been linked with transcriptional program of ribosome biogenesis, as the expression of over $75 \%$ of the factors that are involved in this process have been shown to be controlled by p70S6K in mouse (Chauvin et al., 2013). Ribosome biogenesis is essential for protein synthesis, and has been linked with cell growth and cell cycle control. In addition, both RPS6 and p70S6K have been demonstrated as important regulators of translation processes of mRNAs containing a polypyrimidine tract at their 5' end, also known as 5'TOP mRNAs (Hutchinson et al., 2011; Pende et al., 2004). These mRNAs mainly encode components of the translational apparatus, including ribosomal proteins, translation elongation factors, and poly $(A)$ - binding protein (PABP) (Topisirovic and Sonenberg, 2011). Therefore, the TBTO-mediated dephosphorylation of RPS6 and p70S6K strongly suggest that TBTO suppressed the process of ribosome biogenesis and protein synthesis.

Our transcriptomics data of TBTO revealed similar results (Fig. 4), since $6 \mathrm{~h}$ of TBTO treatment was previously shown to downregulate the genes involved in biogenesis and quality control of the ribosomes, including RRP9, NOP58, NOP56, RPP30, RPP38, RPP40, EXOSC2, EXOSC3, and EXOSC7 (Fig. 5C) (Chauvin et al., 2013; Sloan, 2012). In addition, we also observed downregulation of several mitochondrial ribosomal proteins (MRPs) by TBTO treatment (Fig. 5C, Supplementary fig. 2C). Mitochondrial ribosomal proteins are directly involved in the process of mitochondrial protein synthesis, which is essential for maintaining the integrity of mitochondria (Takeuchi and Ueda, 2003). Taken together, the protein phosphorylation and transcriptome results indicate that TBTO does not only inhibit transcription of cytoplasmatic ribosomal RNAs but also of mitochondrial ribosomal RNAs. These findings are in agreement with the recently published results from our lab that $200 \mathrm{nM}$ of TBTO treatment downregulated the expression levels of genes involved in rRNA and tRNA processing and ribosome biogenesis in Jurkat cells (Schmeits et al., 2014). The present study further demonstrated 
that these effects can already be observed when TBTO is administered at a lower concentration (100 $\mathrm{nM})$.

Besides the effect on ribosome biogenesis, our microarray data also indicated that TBTO affected cell migration since the gene set "leukocyte migration" was upregulated. To validate this, we performed a trans-well based chemotoxis assay which demonstrated that TBTO inhibited CXCL12 induced cell migration. Therefore, the observed upregulation of gene set "leukocyte migration" could be an adaptive response to counteract the inhibition of the migration ability. It can be envisaged that at higher concentrations or after a longer exposure time, these genes would be downregulated.

In summary, by applying a phosphoproteome profiling based approach, we identified the mTOR signaling pathway as a major cellular pathway commonly affected in the Jurkat $\mathrm{T}$ cell line by immunotoxicants. Validation experiments with the model immunotoxicant TBTO and immunosuppressive drug rapamycin indicated that although a number of immunotoxicants modulated the p70S6K pathway, the underlying mechanisms were chemical-dependent. In addition, both transcriptome and protein phosphorylation data revealed an effect of TBTO on ribosome biogenesis and cell chemotaxis.

\section{Funding information}

This study was financially supported by the Netherlands Genomics Initiative, the Netherlands Toxicogenomics Centre (grant 05060510). 


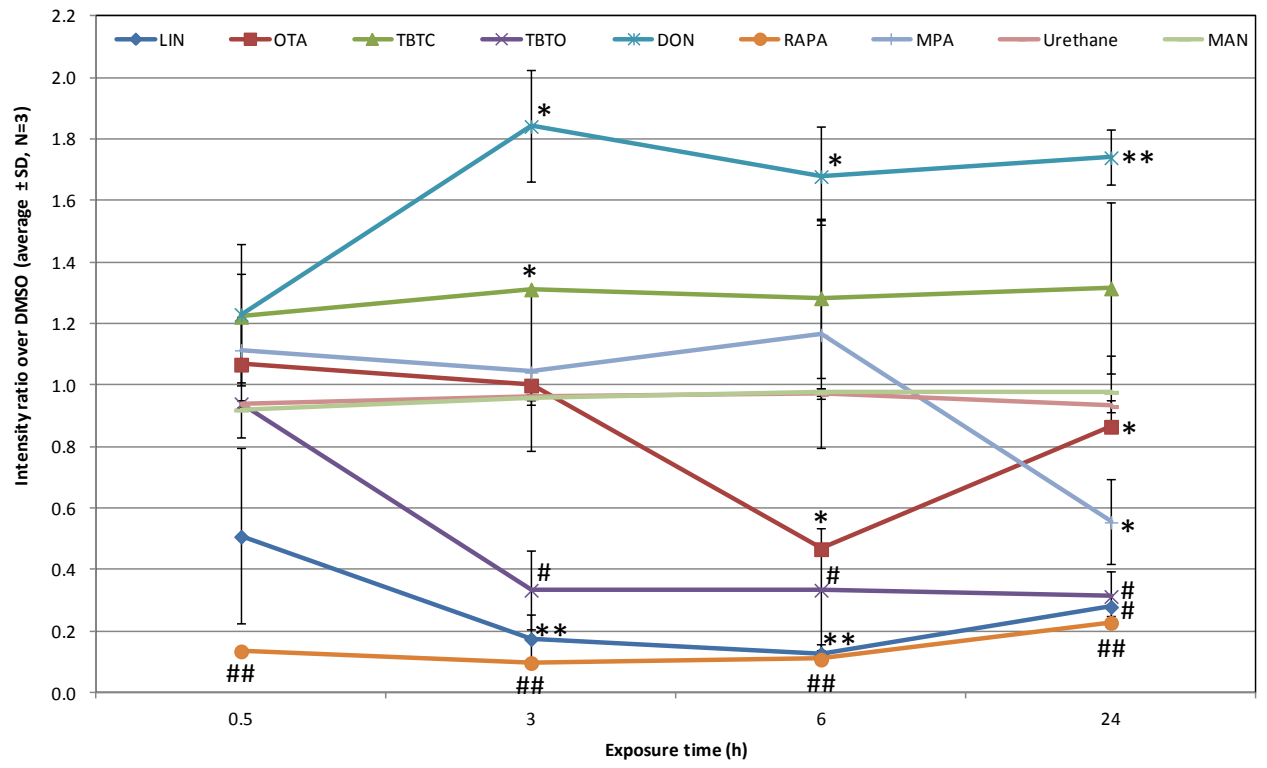

Supplementary fig. 1. Effects of nine compounds on the phosphorylation of RPS6 by antibody array analysis. Jurkat $T$ cells were exposed to five immunotoxicants (tributyltin oxide (TBTO), tributyltin chloride (TBTC), lindane (LIN), ochratoxin A (OTA), and deoxynivalenol (DON)), two immunosuppressive drugs (rapamycin (RAPA) and mycophenolic acid (MPA)), and two nonimmunotoxicants (urethane and mannitol (MAN)) for 0.5, 3, 6, and $24 \mathrm{~h}$. The average results of the expression levels of phosphorylated RPS6 (Ser235/6) from three independent experiments with PathScan RTK antibody arrays are shown in the figure. *: $p<0.05 ;{ }^{* *}: p<0.01, \#: p<0.005, \#$ : $p<0.001$, two sample equal variance $T$ test, two tailed. 

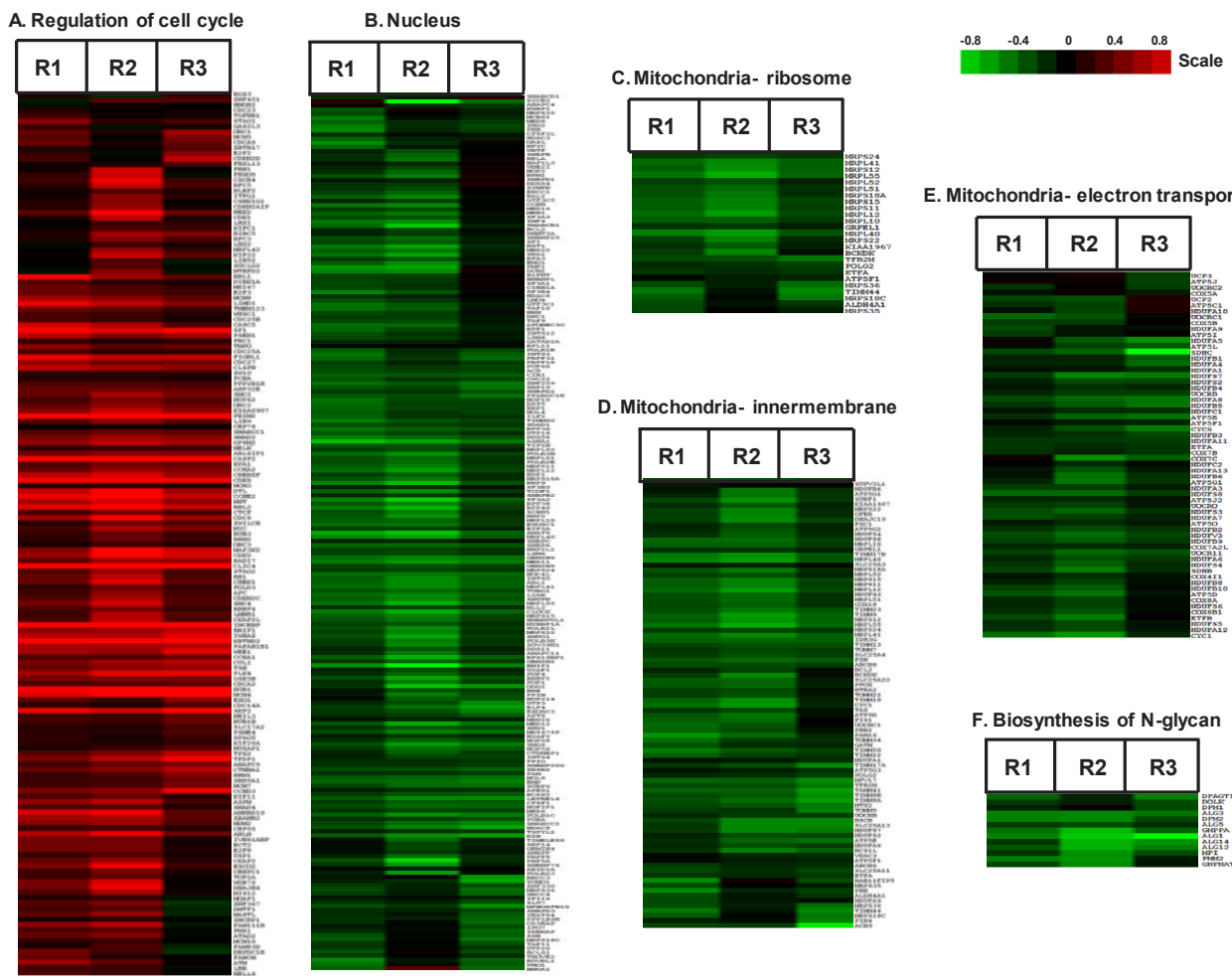

D. Mitochondria- innermembrane
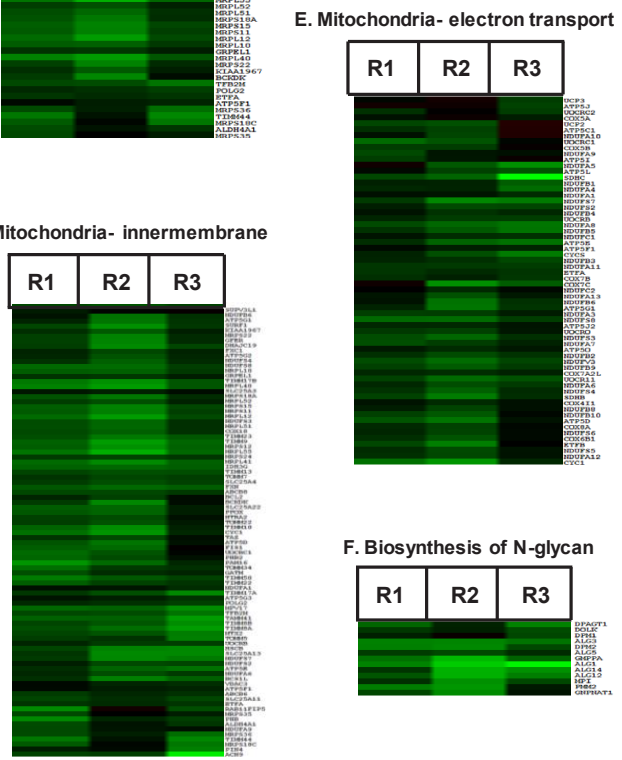

Supplementary fig. 2. Effects of TBTO on the expression levels of genes involved in regulation of cell cycle, nucleus, mitochondria- ribosome, mitochondria- innermembrane, mitochondria- electron transport, and biosynthesis of $\mathrm{N}$-glycan. The heat maps visualize the effects of TBTO exposure on the expression levels of genes involved in six different biological processes in Jurkat cells. Upon exposure for 6 , TBTO upregulated genes that are involved in regulation of cell cycle $(A)$, and downregulated the genes that are invovled in nucleus $(B)$, mitochondria- ribosome (C), mitochondria- innermembrane (D), mitochondria- electron transport $(E)$, and biosynthesis of $\mathrm{N}$-glycan (F). The heatmaps show 2 log ratios of TBTO vs. average of DMSO controls (in three biological replicates, $R 1$ to $R 3)$. Green represents downregulation, red upregulation, and black no regulation. The color intensity is related to the $2 \log$ ratio and is indicated by the bar. 


\section{Reference}

Alessi, D. R., James, S. R., Downes, C. P., Holmes, A. B., Gaffney, P. R. J., Reese, C. B., and Cohen, P. (1997). Characterization of a 3-phosphoinositide-dependent protein kinase which phosphorylates and activates protein kinase Ba. Current Biology 7(4), 261-269.

Araujo, J., and Logothetis, C. (2010). Dasatinib: A potent SRC inhibitor in clinical development for the treatment of solid tumors. Cancer Treatment Reviews 36(6), 492-500.

Bellacosa, A., Kumar, C. C., Cristofano, A. D., Testa, J. R., George, F. V. W., and George, K. (2005). Activation of AKT kinases in cancer: implications for therapeutic targeting. In Advances in Cancer Research (Vol. Volume 94, pp. 29-86. Academic Press.

Bełtowski, J., Wójcicka, G., Trzeciak, J., and Marciniak, A. (2006). H2O2 and Src-dependent transactivation of the EGF receptor mediates the stimulatory effect of leptin on renal ERK and $\mathrm{Na}+$, K+-ATPase. Peptides 27(12), 3234-3244.

Berg, J., Tymoczko, J., and Stryer, L. (2002). Defects in signaling pathways can lead to cancer and other diseases. In Biochemistry. 5th Edition. New York: W. H. Freeman, Section 15.5.

Brazil, D. P., and Hemmings, B. A. (2001). Ten years of protein kinase B signalling: a hard Akt to follow. Trends in Biochemical Sciences 26(11), 657-664.

Byeon, S. E., Yi, Y. S., Oh, J., Yoo, B. C., Hong, S., and Cho, J. Y. (2012). The role of Src kinase in macrophage-mediated inflammatory responses. Mediators of Inflammation 2012, 18.

Chaturvedi, D., Gao, X., Cohen, M. S., Taunton, J., and Patel, T. B. (2009). Rapamycin induces transactivation of the EGFR and increases cell survival. Oncogene 28(9), 1187-1196.

Chauvin, C., Koka, V., Nouschi, A., Mieulet, V., Hoareau-Aveilla, C., Dreazen, A., Cagnard, N., Carpentier, W., Kiss, T., Meyuhas, O., and Pende, M. (2013). Ribosomal protein S6 kinase activity controls the ribosome biogenesis transcriptional program. Oncogene 33(4), 474-483.

Chen, H. (2005). Boyden chamber assay. Methods Mol Biol 294, 15-22.

Chung, J., Kuo, C. J., Crabtree, G. R., and Blenis, J. (1992). Rapamycin-FKBP specifically blocks growth-dependent activation of and signaling by the $70 \mathrm{kd}$ S6 protein kinases. Cell 69(7), 1227-1236.

Cortes, J. E., Kim, D.-W., Kantarjian, H. M., Brümmendorf, T. H., Dyagil, I., Griskevicius, L., Malhotra, H., Powell, C., Gogat, K., Countouriotis, A. M., and Gambacorti-Passerini, C. (2012). Bosutinib versus imatinib in newly diagnosed chronic-phase chronic myeloid leukemia: results from the BELA trial. Journal of Clinical Oncology 30(28), 3486-3492.

Davila, D. R. (1999). Protein tyrosine kinase activation by polycyclic aromatic hydrocarbons in human HPB-ALL T cells. Journal of Toxicology and Environmental Health, Part A 56(4), 249-261.

Fayard, E., Tintignac, L. A., Baudry, A., and Hemmings, B. A. (2005). Protein kinase B/Akt at a glance. Journal of Cell Science 118(24), 5675-5678.

Guo, J., Wu, H. W., Hu, G., Han, X., De, W., and Sun, Y. J. (2006). Sustained activation of Src-family tyrosine kinases by ischemia: A potential mechanism mediating extracellular signal-regulated kinase cascades in hippocampal dentate gyrus. Neuroscience 143(3), 827-836.

Haar, E. V., Lee, S.-i., Bandhakavi, S., Griffin, T. J., and Kim, D.-H. (2007). Insulin signalling to mTOR mediated by the Akt/PKB substrate PRAS40. Nat Cell Bio/ 9(3), 316-323. 
Hay, N., and Sonenberg, N. (2004). Upstream and downstream of mTOR. Genes \& Development 18(16), 1926-1945.

Hemmings, B. A., and Restuccia, D. F. (2012). PI3K-PKB/Akt pathway. Cold Spring Harbor Perspectives in Biology 4(9).

Hirai, I., Ebara, M., Nakanishi, S., Yamamoto, C., Sasaki, T., Ikuta, K., and Yamamoto, Y. (2013). Jurkat cell proliferation is suppressed by Chlamydia (Chlamydophila) pneumoniae infection accompanied with attenuation of phosphorylation at Thr389 of host cellular p70S6K. Immunobiology 218(4), 527-532.

Holz, M. K., and Blenis, J. (2005). Identification of S6 kinase 1 as a novel mammalian target of rapamycin (mTOR)-phosphorylating kinase. Journal of Biological Chemistry 280(28), 26089-26093.

Homsi, J., Cubitt, C. L., Zhang, S., Munster, P. N., Yu, H., Sullivan, D. M., Jove, R., Messina, J. L., and Daud, A. I. (2009). Src activation in melanoma and Src inhibitors as therapeutic agents in melanoma. Melanoma Research 19(3), 167-175.

$\mathrm{Hu}, \mathrm{X} ., \mathrm{Wu}, \mathrm{X} ., \mathrm{Xu}$, J., Zhou, J., Han, X., and Guo, J. (2009). Src kinase up-regulates the ERK cascade through inactivation of protein phosphatase $2 \mathrm{~A}$ following cerebral ischemia. $B M C$ Neuroscience 10(1), 74.

Hutchinson, J. A., Shanware, N. P., Chang, H., and Tibbetts, R. S. (2011). Regulation of ribosomal protein $\mathrm{S} 6$ phosphorylation by casein kinase 1 and protein phosphatase 1. Journal of Biological Chemistry 286(10), 8688-8696.

Khalaileh, A., Dreazen, A., Khatib, A., Apel, R., Swisa, A., Kidess-Bassir, N., Maitra, A., Meyuhas, O., Dor, Y., and Zamir, G. (2013). Phosphorylation of ribosomal protein S6 attenuates DNA damage and tumor suppression during development of pancreatic cancer. Cancer Research 73(6), 1811-1820.

Korade-Mirnics, Ž., and Corey, S. J. (2000). Src kinase-mediated signaling in leukocytes. Journal of Leukocyte Biology 68(5), 603-613.

Meyuhas, O. (2000). Synthesis of the translational apparatus is regulated at the translational level. European Journal of Biochemistry 267(21), 6321-6330.

Meyuhas, O., and Kwang, W. J. (2008). Chapter 1 Physiological roles of ribosomal protein S6: one of its kind. In International Review of Cell and Molecular Biology (Vol. 268), pp. 1-37. Academic Press.

Mikasova, L., De Rossi, P., Bouchet, D., Georges, F., Rogemond, V., Didelot, A., Meissirel, C., Honnorat, J., and Groc, L. (2012). Disrupted surface cross-talk between NMDA and Ephrin-B2 receptors in anti-NMDA encephalitis. Brain 135(5), 1606-1621.

Min, Y. H., Eom, J. I., Cheong, J. W., Maeng, H. O., Kim, J. Y., Jeung, H. K., Lee, S. T., Lee, M. H., Hahn, J. S., and Ko, Y. W. (2003). Constitutive phosphorylation of Akt//PKB protein in acute myeloid leukemia: its significance as a prognostic variable. Leukemia 17(5), 995-997.

Mograbi, B., Corcelle, E., Defamie, N., Samson, M., Nebout, M., Segretain, D., Fénichel, P., and Pointis, G. (2003). Aberrant Connexin 43 endocytosis by the carcinogen lindane involves activation of the ERK/mitogen-activated protein kinase pathway. Carcinogenesis 24(8), 1415-1423.

Munro, K. M., Dixon, K. J., Gresle, M. M., Jonas, A., Kemper, D., Doherty, W., Fabri, L. J., Owczarek, C. M., Pearse, M., Boyd, A. W., Kilpatrick, T. J., Butzkueven, H., and Turnley, A. M. (2013). EphA4 
receptor tyrosine kinase Is a modulator of onset and disease severity of experimental autoimmune encephalomyelitis (EAE). PLoS ONE 8(2), e55948.

Osman, A. M., and van Loveren, H. (2012). Phosphoproteomic analysis of mouse thymoma cells treated with tributyltin oxide: TBTO affects proliferation and energy sensing pathways. Toxicological Sciences 126(1), 84-100.

Osman, A. M., and van Loveren, H. (2013). The immunosuppressant tributyltin oxide blocks the mTOR pathway, like rapamycin, albeit by a different mechanism. Journal of Applied Toxicology, 34: 13611367.

Patel, R., and Mohan, C. (2005). PI3K/AKT signaling and systemic autoimmunity. Immunol. Res. 31(1), 47-55.

Pende, M., Um, S. H., Mieulet, V., Sticker, M., Goss, V. L., Mestan, J., Mueller, M., Fumagalli, S., Kozma, S. C., and Thomas, G. (2004). S6K1-/-/S6K2-/- mice exhibit perinatal lethality and rapamycin-sensitive 5'-terminal oligopyrimidine mRNA translation and reveal a mitogen-activated protein kinase-dependent S6 kinase pathway. Molecular and Cellular Biology 24(8), 3112-3124.

Robbins, S. M., Quintrell, N. A., and Bishop, J. M. (2000). Mercuric chloride activates the Src-family protein tyrosine kinase, Hck in myelomonocytic cells. European Journal of Biochemistry 267(24), 7201-7208.

Robinson, D., Wu, Y., and Lin, S. (2000). The protein tyrosine kinase family of the human genome. Oncogene 19(49), 5548-5557.

Roux, P. P., Shahbazian, D., Vu, H., Holz, M. K., Cohen, M. S., Taunton, J., Sonenberg, N., and Blenis, J. (2007). RAS/ERK signaling promotes site-specific ribosomal protein $\mathrm{S} 6$ phosphorylation via RSK and stimulates cap-dependent translation. Journal of Biological Chemistry 282(19), 14056-14064. Salmond, R. J., Emery, J., Okkenhaug, K., and Zamoyska, R. (2009a). MAPK, phosphatidylinositol 3kinase, and mammalian target of rapamycin pathways converge at the level of ribosomal protein S6 phosphorylation to control metabolic signaling in CD8 T cells. The Journal of Immunology 183(11), 7388-7397.

Salmond, R. J., Filby, A., Qureshi, I., Caserta, S., and Zamoyska, R. (2009b). T-cell receptor proximal signaling via the Src-family kinases, Lck and Fyn, influences T-cell activation, differentiation, and tolerance. Immunological Reviews 228(1), 9-22.

Sarbassov, D. D., Guertin, D. A., Ali, S. M., and Sabatini, D. M. (2005). Phosphorylation and regulation of Akt/PKB by the Rictor-mTOR complex. Science 307(5712), 1098-1101.

Schmeits, P. C. J., Katika, M. R., Peijnenburg, A. A. C. M., van Loveren, H., and Hendriksen, P. J. M. (2014). DON shares a similar mode of action as the ribotoxic stress inducer anisomycin while TBTO shares ER stress patterns with the ER stress inducer thapsigargin based on comparative gene expression profiling in Jurkat T cells. Toxicology Letters 224(3), 395-406.

Shaffer, A. L., Rosenwald, A., Hurt, E. M., Giltnane, J. M., Lam, L. T., Pickeral, O. K., and Staudt, L. M. (2001). Signatures of the immune response. Immunity 15(3), 375-385.

Shan, X., Balakir, R., Criado, G., Wood, J. S., Seminario, M.-C., Madrenas, J., and Wange, R. L. (2001). ZAP-70-independent Ca2+ mobilization and Erk activation in Jurkat T cells in response to TCell antigen receptor ligation. Molecular and Cellular Biology 21(21), 7137-7149. 
Shannon, P., Markiel, A., Ozier, O., Baliga, N. S., Wang, J. T., Ramage, D., Amin, N., Schwikowski, B., and Ideker, T. (2003). Cytoscape: a software environment for integrated models of biomolecular interaction networks. Genome Research 13(11), 2498-2504.

Shao, J., Katika, M. R., Schmeits, P. C. J., Hendriksen, P. J. M., van Loveren, H., Peijnenburg, A. A. C. M., and Volger, O. L. (2013). Toxicogenomics-based identification of mechanisms for direct immunotoxicity. Toxicological Sciences 135(2), 328-346.

Sharfe, N., Freywald, A., Toro, A., Dadi, H., and Roifman, C. (2002). Ephrin stimulation modulates T cell chemotaxis. Eur. J. Immunol. 32(12), 3745-3755.

Sloan, K. (2012). The exosome and human ribosome biogenesis. PhD, Newcastle University, Newcastle University.

Smid, M., Dorssers, L. C. J., and Jenster, G. (2003). Venn Mapping: clustering of heterologous microarray data based on the number of co-occurring differentially expressed genes. Bioinformatics 19(16), 2065-2071.

Šulić, S., Panić, L., Barkić, M., Merćep, M., Uzelac, M., and Volarević, S. (2005). Inactivation of S6 ribosomal protein gene in $\mathrm{T}$ lymphocytes activates a p53-dependent checkpoint response. Genes \& Development 19(24), 3070-3082.

Takeuchi, N., and Ueda, T. (2003). Down-regulation of the mitochondrial translation system during terminal differentiation of HL-60 cells by 12-o-tetradecanoyl-1-phorbol-13-acetate: comparison with the cytoplasmic translation system. Journal of Biological Chemistry 278(46), 45318-45324.

Thomas, S. M., and Brugge, J. S. (1997). Cellular functions regulated by Src family kinases. Annual Review of Cell and Developmental Biology 13(1), 513-609.

Topisirovic, I., and Sonenberg, N. (2011). Translational control by the eukaryotic ribosome. Cell 145(3), 333-334.

Tworkoski, K., Singhal, G., Szpakowski, S., Zito, C. I., Bacchiocchi, A., Muthusamy, V., Bosenberg, M., Krauthammer, M., Halaban, R., and Stern, D. F. (2011). Phosphoproteomic screen identifies potential therapeutic targets in melanoma. Molecular Cancer Research 9(6), 801-812.

Vila-Coro, A. J., Rodriguez-Frade, J. M., Martin de Ana, A., Moreno-Ortiz, M. C., Martinez-A, C., and Mellado, M. (1999). The chemokine SDF-1a triggers CXCR4 receptor dimerization and activates the JAK/STAT pathway. The FASEB Journal 13(13), 1699-1710.

Zwaan, C. M., Rizzari, C., Mechinaud, F., Lancaster, D. L., Lehrnbecher, T., van der Velden, V. H. J., Beverloo, B. B., den Boer, M. L., Pieters, R., Reinhardt, D., Dworzak, M., Rosenberg, J., Manos, G., Agrawal, S., Strauss, L., Baruchel, A., and Kearns, P. R. (2013). Dasatinib in children and adolescents with relapsed or refractory leukemia: results of the CA180-018 phase I dose-escalation study of the innovative therapies for children with cancer consortium. Journal of Clinical Oncology 31(19), 24602468. 


\section{CHAPTER 4}

\section{Inhibition of CXCL12-mediated chemotaxis of Jurkat cells by direct immunotoxicants}

Jia Shao, Inge Stout, Oscar L. Volger, Peter J. M. Hendriksen, Henk van Loveren, and Ad A. C. M. Peijnenburg

In preparation. 


\begin{abstract}
Directional migration of cells to specific locations is required in tissue development, wound healing, and immune responses. Immune cell migration plays a crucial role in both innate and adaptive immunity. Chemokines are small pro-inflammatory chemoattractants that control the migration of leukocytes. In addition, they are also involved in other immune processes such as lymphocyte development and immune pathology. In a previous toxicogenomics study using the Jurkat T-cell line, we have shown that the model immunotoxicant TBTO inhibited chemotaxis towards the chemokine CXCL12. In the present work, we aimed at identifying other immunotoxicants with chemotaxismodulating properties based on their effects on mRNA expression of genes that are known to be related with cell migration. With this analysis, seven immunotoxicants were identified as potential chemotaxis modulators, of which five $\left(\mathrm{CoCl}_{2}, \mathrm{MeHg}\right.$, ochratoxin $\mathrm{A}$, S9-treated ochratoxin A and TBTO) were confirmed as chemotaxis inhibitor in an in vitro trans-well chemotaxis assay using the chemokine CXCL12. The transcriptome data of the five compounds together with previously obtained protein phosphorylation profiles for two out of five compounds (i.e., ochratoxin A and TBTO) revealed that the mechanisms behind the chemotaxis inhibition are different for these immunotoxicants. Moreover, the mTOR inhibitor rapamycin had no effect on the chemotaxis of Jurkat cells, indicating that the mTOR pathway is not involved in CXCL12-mediated chemotaxis of Jurkat cells, which is opposite to the findings on human primary $T$ cells. Thus, the results obtained from the chemotaxis assay conducted with Jurkat cells might not fully represent the results obtained with human primary $T$ cells. Despite this difference, the present study indicated that some compounds may exert their immunotoxic effects through inhibition of CXCL12-mediated chemotaxis.
\end{abstract}




\section{Introduction}

Directional cell migration is a tightly controlled process, which is crucial for numerous biological processes including tissue development, wound healing, and immune responses. Immune cell migration is now recognized to be intimately associated with both innate and adaptive immunity (Madri and Graesser, 2000). For instance, leukocyte extravasation from the circulation to the site of affected tissue is essential for the initiation of inflammatory responses (Newton and Dixit, 2012), while the local inflammatory microenvironment plays a critical role in regulating the migration of maturing dendritic cells to secondary lymphoid organs, where they activate antigen specific naive $T$ lymphocytes (Del Prete et al., 2007). Migration of leukocytes is mediated by chemokines which are small proinflammatory chemoattractants and exert their effect by binding to seven-transmembrane $\mathrm{G}$ proteincoupled receptors. They have been demonstrated not only to regulate immune cell migration and adhesion, but also to play a vital role in certain immune processes that do not directly involve leukocyte migration, such as lymphocyte development and immune pathology (Moser et al., 2004).

Among the various chemokines, $\mathrm{CXCL} 12$ (initially called stromal cell derived factor $1-\alpha$, or SDF-1 $\alpha$ ) is a highly efficient chemoattractant for major types of immune cells (Munk et al., 2011). Signalling through CXCL12 and its cognate receptor, chemokine receptor 4 (CXCR4), has been implicated in different processes such as immune regulation, inflammation progression, and cancer development (Wojcechowskyj et al., 2011). Deletion of CXCL12 or CXCR4 is lethal in mice and causes disruption in lymphopoiesis and bone marrow myolopoiesis (Nagasawa et al., 1996; Zou et al., 1998). It had been believed for many years that CXCR4 was the only receptor for CXCL12 until the discovery of another receptor CXCR7 (Balabanian et al., 2005). However, in contrast to CXCR4, deletion of CXCR7 does not affect hematopoiesis in mice but instead induces cardiovascular defects, which highlights the distinct roles of CXCR4 and CXCR7 in regulating CXCL12-mediated effects with CXCR7 being more involved in cardiac development (Sierro et al., 2007; Gerrits et al., 2008). In addition, it has been revealed that CXCL12 does not induce signaling through CXCR7. Instead, CXCR7 forms heterodimers with CXCR4 and subsequently enhances the cellular responsiveness to CXCL12, leading to enhanced chemotaxis towards CXCL12 (Sierro et al., 2007; Levoye et al., 2009).

Immune cell chemotaxis induced by CXCL12 is achieved by membrane remodelling and cytoskeletal rearrangement including actin polymerization (Vicente-Manzanares et al., 2005; Voermans et al., 2001), which is the result of a cascade of intracellular signaling events including JAK/STAT, PI3K, and MAPK/ ERK pathways (Vila-Coro et al., 1999; Tilton et al., 2000; Ticchioni et al., 2002; Kumar et al., 2006). Src family kinases have also been indicated to be involved in the chemotaxis of $T$ cells in response to CXCL12, as the Src kinase inhibitor PP2 partially blocks this process (Okabe et al., 2002). More recently, it has been suggested that also the mTOR signaling pathway is involved in CXCL12mediated chemotaxis, as the mTOR inhibitor rapamycin significantly inhibits the chemotaxis of both primary human resting T cells and the human leukemia T cell line CEM (Munk et al., 2011). 
In a previous toxicogenomics study using the Jurkat $T$ cell line, we have shown that the model immunotoxicant TBTO inhibited CXCL12-induced chemotaxis (Shao et al., in preparation). In the present work, based on the alteration of mRNA expression of genes that are known to be related with cell migration in Jurkat cells, we identified seven immunotoxicants (including TBTO) as potential chemotaxis modulators. Subsequently, we investigated the effects of these immunotoxicants on the CXCL12-mediated chemotaxis of Jurkat cells in an in vitro trans-well chemotaxis assay. Five out of the seven compounds inhibited the chemotaxis of Jurkat cells, being $\mathrm{CoCl}_{2}, \mathrm{MeHg}$, ochratoxin $\mathrm{A}, \mathrm{S} 9$ treated ochratoxin A, and TBTO. The transcriptome data of these five compounds and the effects on protein phosphorylation for two out of the five compounds (i.e., ochratoxin A and TBTO) indicated that these immunotoxicants inhibit chemotaxis via different mechanisms. Furthermore, the results obtained with the mTOR inhibitor rapamycin indicated that, unlike human primary T cells, the mTOR pathway is not involved in CXCL12-mediated chemotaxis of Jurkat cells. Despite this difference, the data of the present study showed that inhibition of CXCL12-mediated chemotaxis might be involved in the immunotoxic effects of some immunotoxicants.

\section{Materials and Methods}

Chemicals. Selection criteria and supplier information for the test compounds were described before (Shao et al., 2013). In total three compounds were known to be bio-tranformed in vivo, which results in the production of immunosuppressive metabolites, being benzo[a]pyrene (BaP), cyclophosphamide (CP), and ochratoxin A (OTA) (Carlson et al., 2004; Creppy et al., 1983; Ekhart et al., 2009). Therefore, these three compounds were subjected to in vitro metabolic activation using a human liver S9 fraction (BD Bioscience, Breda, The Netherlands). The metabolic activation system and incubation protocol were described before (Shao et al., 2013).

Cell culture. The Jurkat cell line was obtained from the American Type Culture Collection (ATCC). They were grown in RPMI-1640 medium supplemented with $10 \%$ heat inactivated fetal calf serum (FCS), $2 \mathrm{mM}$ glutamine, $1 \mathrm{mM}$ sodium pyruvate, $1 \mathrm{mM}$ nonessential amino acids, $100 \mathrm{U} / \mathrm{ml}$ penicillin, and $100 \mu \mathrm{g} / \mathrm{ml}$ streptomycin. Cells were cultured at $37^{\circ} \mathrm{C}$ with $5 \% \mathrm{CO}_{2}$ in a humidified atmosphere. Refreshment of the medium was done every 2 days.

Gene set enrichment analysis. Gene set enrichment analysis (GSEA) was performed on the transcriptome data set generated in a previous study, in which Jurkat cells were exposed for $h$ to subcytotoxic concentrations of 31 compounds and then subjected to microarray analysis (Shao et al., 2013). The method for GSEA analysis was also described in the same study (Shao et al., 2013). In the present study, we used the gene set which was a combination of the gene set "cell migration" from Gene Ontology consortium (downloaded from MSigDB, 
http://www.broadinstitute.org/gsea/msigdb/index.jsp) and the gene set "leukocyte_transendothelial_migration" from KEGG (downloaded from KEGG, http://www.genome.jp/kegg). In total, this combined gene set contains 202 genes. 198 out of the 202 genes remained in the transcriptome data set after custom CDF filtering of the raw data (Shao et al., 2013). Therefore, the GSEA analysis was performed using the gene set containing 198 genes (Supplementary Table 1). Compounds with a $p$ value $<0.05$ and an FDR value $<0.25$ were considered as potential chemotaxis modulators.

Chemotaxis assay. The chemotaxis of Jurkat cells towards the chemokine CXCL12 was assessed using 24-well trans-well chambers with $5 \mu \mathrm{m}$ pores (Corning Life Science BV, Amsterdam, The Netherlands). This assay was performed according to Boyden chamber assay (Chen, 2005) with minor modifications. Jurkat cells (passage number 16-19) were seeded in $900 \mu \mathrm{l}$ medium per well in 12-well plates $(60,000$ cells/well). After growing the cells for $20 \mathrm{~h}$, exposure was initiated by adding $100 \mu \mathrm{l}$ medium containing either test chemicals or DMSO. The concentrations for all the test chemicals were subcytotoxic for up to at least $24 \mathrm{~h}$, and were the same as the ones used in the transcriptomics experiment (Shao et al., 2013). The final DMSO concentration was $0.1 \%(\mathrm{v} / \mathrm{v})$ for all samples. Subsequently, cells were exposed for $24 \mathrm{~h}$. In the last $2 \mathrm{~h}$ of exposure, cells were counted and transferred to the apical compartments of the trans-well system ( $150 \mu \mathrm{l}$, in total $1.5 \times 10^{5}$ cells/ insert). $500 \mu \mathrm{l}$ of medium containing $25 \mathrm{ng} / \mathrm{ml}$ of CXCL12 (Biosource, Camarillo, CA) and either the same exposure concentration of test chemical or $0.1 \%$ DMSO were added to the basolateral compartments. Migration was allowed for $2 \mathrm{~h}$ at $37^{\circ} \mathrm{C}$. After the experiments, cells that migrated into the basolateral compartments were counted by a Z2 Coulter Particle Count and Size Analyzer (Beckman Coulter Netherland BV, Woerden, The Netherlands). The percentage of the migrated cells were then calculated and compared to that of DMSO.

Real time quantitative PCR. The primers for qRT-PCR were designed by ourselves using BEACON designer 7.0 software (Premier Biosoft International, Palo Alto, CA), close to the poly-A tail and intron spanning where possible. The primer sequences are shown in Table 1. RPLP0 and GAPDH were used as reference genes. cDNA samples were synthesized from the same RNA samples as used for the microarray experiment using miScript Reverse Transcription kit according to the manufacturer's protocol (Qiagen, Venlo, Netherlands). qRT-PCR reactions were performed on Bio-Rad CFX96 real time detection system (Veenendaal, The Netherlands). The reaction mixture consisted of $2 \mu \mathrm{l}$ of cDNA, $8.5 \mu \mathrm{l}$ of SYBRgreen super mix, $2.5 \mu \mathrm{l}$ of RNase free water, $1 \mu \mathrm{l}$ of forward and $1 \mu \mathrm{l}$ of reverse primer. The reaction mixtures were incubated for $3 \mathrm{~min}$ at $95^{\circ} \mathrm{C}$ and followed by 45 cycles of amplification. The PCR settings were as follows: denaturation $10 \mathrm{~s}$ at $95^{\circ} \mathrm{C}$, annealing $30 \mathrm{~s}$ at $60^{\circ} \mathrm{C}$, and extension $10 \mathrm{~s}$ at $95{ }^{\circ} \mathrm{C}$ after the last cycle of the PCR reaction. Relative expression levels were calculated using Bio-Rad CFX manager v2.0. The significance of different expression between treated and control samples was determined by two sample equal variance T-test. 
TABLE 1. Primer sequences used in $q R T-P C R$ verification experiments.

\begin{tabular}{c|c|c|c}
\hline Accession Number & Gene & Oligo sequence $\left(\mathbf{5}^{\prime} \rightarrow \mathbf{3}^{\prime}\right)$ sense & Oligo sequence $\left(\mathbf{5}^{\prime} \rightarrow \mathbf{3}^{\prime}\right)$ anti sense \\
\hline NM_001008540 & CXCR4 & AGTGAGGCAGATGACAGATA & CAATACCAGGCAGGATAAGG \\
NM_001002 & RPLP0 & ATCATCAACGGGTACAAACGAGTC & GCAGATGGATCAGCCAAGAAGG \\
NM_002046 & GAPDH & AGGTCGGAGTCAACGGATTTGG & GCTCCTGGAAGATGGTGATGGG \\
\hline
\end{tabular}

\section{Results}

Gene set enrichment analysis (GSEA) identified potential chemotaxis modulators

GSEA was used to assess the effects of 31 compounds on a combined gene set consisting of 198 genes that are known to be related to cell migration. This gene set was found to be significantly affected (upregulated) by seven immunotoxicants, i.e., S9-treated benzo[a]pyrene, $\mathrm{CoCl}_{2}, \mathrm{MeHg}$, ochratoxin A, S9-treated ochratoxin A, TBTC, and TBTO, when considering a $p$ value $<0.05$ and a FDR value $<0.25$ as the cut off (Table 2). The expression levels of the 198 genes in response to $6 \mathrm{~h}$ exposure to the seven compounds are shown in Figure 1. For comparison, the effects of two nonimmunotoxic control compounds, being mannitol and urethane, on the expression levels of these 198 genes were also included in the heatmap (Figure 1). Genes that are commonly upregulated by these seven immunotoxicants include the ones encoding three leukocyte integrins, i.e. ITGAL (alias CD11a), ITGA4, and ITGB2 (alias CD18), two Rho-associated, coiled-coil containing protein kinases, i.e. ROCK1 and ROCK2, and three catalytic subunits of PI3-kinase (PI3K), i.e. PIK3CA, PIK3CB, and PIK3CG (Figure 1, enlarged in Supplementary Figure 1A). In addition, a number of genes were specifically regulated by two or three of the seven immunotoxicants. These include genes encoding protein kinase $C$ beta (PRKCB) and extracellular matrix protein, spondin 2 (SPON2) that were upregulated by ochratoxin A and S9-treated ochratoxin A (Figure 1, enlarged in Supplementary Figure 1B). Genes encoding two enzymes involved in sphingolipid metabolism, being sphingosine kinase 1 (SPHK1) and a member of the phosphatidic acid phosphatase family, being phosphatidic acid phosphatase 2a (PPAP2A); two enzymes involved in the formation of inositol 1, 4, 5-trisphosphate (IP3) and diacylglycerol (DAG), being phospholipase C, gamma 1 and 2 (PLCG1 and PLCG2); the receptor for chemokine CXCL12, chemokine receptor 4 (CXCR4); and mitogen-activated protein kinase 12 (MAPK12) were specifically downregulated by ochratoxin A and S9-treated ochratoxin A (Figure 1, enlarged in Supplementary Figure 1C). The gene encoding $\mathrm{S} 100$ calcium binding protein $\mathrm{P}$ (S100P) was upregulated by ochratoxin A and downregulated by S9-treated benzo[a]pyrene (Figure 1, enlarged in Supplementary Figure 1B). The gene encoding Tec family kinase (ITK) was downregulated by ochratoxin A, S9-treated ochratoxin A, and S9 treated benzo[a]pyrene (Figure 1, enlarged in Supplementary Figure 1C). 
TABLE 2. Results of GSEA analysis for 31 compounds using the combined gene set for cell migration.

\begin{tabular}{|c|c|c|c|c|c|c|c|}
\hline Chemical & Abbreviation & ES & NES & $p$ value & FDR & $\begin{array}{l}\text { Potential } \\
\text { chemotaxis } \\
\text { modulator }\end{array}$ & Class \\
\hline Benzo[a]pyrene S9 treated & BaP S9 & 0.26 & 1.20 & 0.04 & 0.04 & \multirow{7}{*}{ Yes } & \multirow{15}{*}{ Immunotoxicants } \\
\hline Cobalt(II) chloride & $\mathrm{CoCl} 2$ & 0.32 & 1.55 & 0.00 & 0.00 & & \\
\hline Methylmercury & $\mathrm{MeHg}$ & 0.26 & 1.29 & 0.02 & 0.02 & & \\
\hline Ochratoxin A & OTA & 0.30 & 1.34 & 0.01 & 0.01 & & \\
\hline Ochratoxin A S9 treated & OTA S9 & 0.31 & 1.46 & 0.00 & 0.00 & & \\
\hline Tributyltin chloride & TВTC & 0.27 & 1.38 & 0.00 & 0.00 & & \\
\hline Bis(tributyltin)oxide & TВТО & 0.32 & 1.58 & 0.00 & 0.00 & & \\
\hline Arsenic trioxide & As2O3 & -0.21 & -0.80 & 0.93 & 0.71 & \multirow{21}{*}{ No } & \\
\hline Benzo[a]pyrene & $\mathrm{BaP}$ & 0.18 & 0.99 & 0.52 & 0.52 & & \\
\hline Dibutyltin chloride & DBTC & 0.22 & 1.13 & 0.14 & 0.14 & & \\
\hline Deoxynivalenol & DON & -0.27 & -1.12 & 0.21 & 0.21 & & \\
\hline Diazinon & $\mathrm{DZN}$ & 0.18 & 0.90 & 0.81 & 0.81 & & \\
\hline Lindane & LIN & 0.24 & 1.06 & 0.24 & 0.24 & & \\
\hline Mono-2-ethylhexyl phthalate & MEHP & -0.16 & -0.67 & 1.00 & 1.00 & & \\
\hline Propanil & PROP & 0.19 & 0.98 & 0.54 & 0.54 & & \\
\hline Cyclophosphamide & $\mathrm{CP}$ & -0.17 & -0.78 & 0.94 & 0.94 & & \multirow{7}{*}{$\begin{array}{c}\text { Immunosuppressive } \\
\text { drug }\end{array}$} \\
\hline Cyclophosphamide S9 treated & CP S9 & 0.24 & 1.08 & 0.24 & 0.24 & & \\
\hline Cyclosporine A & CsA & 0.21 & 1.01 & 0.44 & 0.44 & & \\
\hline Fingolimod & FTY720 & -0.23 & -0.97 & 0.54 & 0.54 & & \\
\hline Mycophenolic acid & MPA & -0.22 & -0.86 & 0.84 & 0.84 & & \\
\hline Predinisolone & PRD & -0.17 & -0.73 & 0.98 & 0.98 & & \\
\hline Rapamycin & Rapa & 0.20 & 0.88 & 0.80 & 0.80 & & \\
\hline Silver nitrate & AgNO3 & -0.20 & -0.90 & 0.70 & 0.70 & & \multirow{6}{*}{$\begin{array}{l}\text { Non-immunotoxic } \\
\text { control chemicals }\end{array}$} \\
\hline Ampicillin & AMP & 0.30 & 1.29 & 0.06 & 0.06 & & \\
\hline Azathioprine & AZA & 0.26 & 1.19 & 0.09 & 0.09 & & \\
\hline Bis(2-ethylhexyl) phthalate & DEHP & 0.23 & 1.15 & 0.14 & 0.14 & & \\
\hline Fluoxetine & $\mathrm{FLX}$ & 0.19 & 0.84 & 0.87 & 0.87 & & \\
\hline Furosemide & FURO & -0.17 & -0.77 & 0.95 & 0.95 & & \\
\hline
\end{tabular}




\begin{tabular}{c|c|c|c|c|c|c|} 
Mannitol & MAN & 0.27 & 1.15 & 0.14 & 0.14 & \\
Sodium citrate & Nacitr & 0.26 & 1.17 & 0.13 & 0.13 & \\
Urethane & Urethane & -0.26 & -1.30 & 0.30 & 0.30 & \\
\hline
\end{tabular}

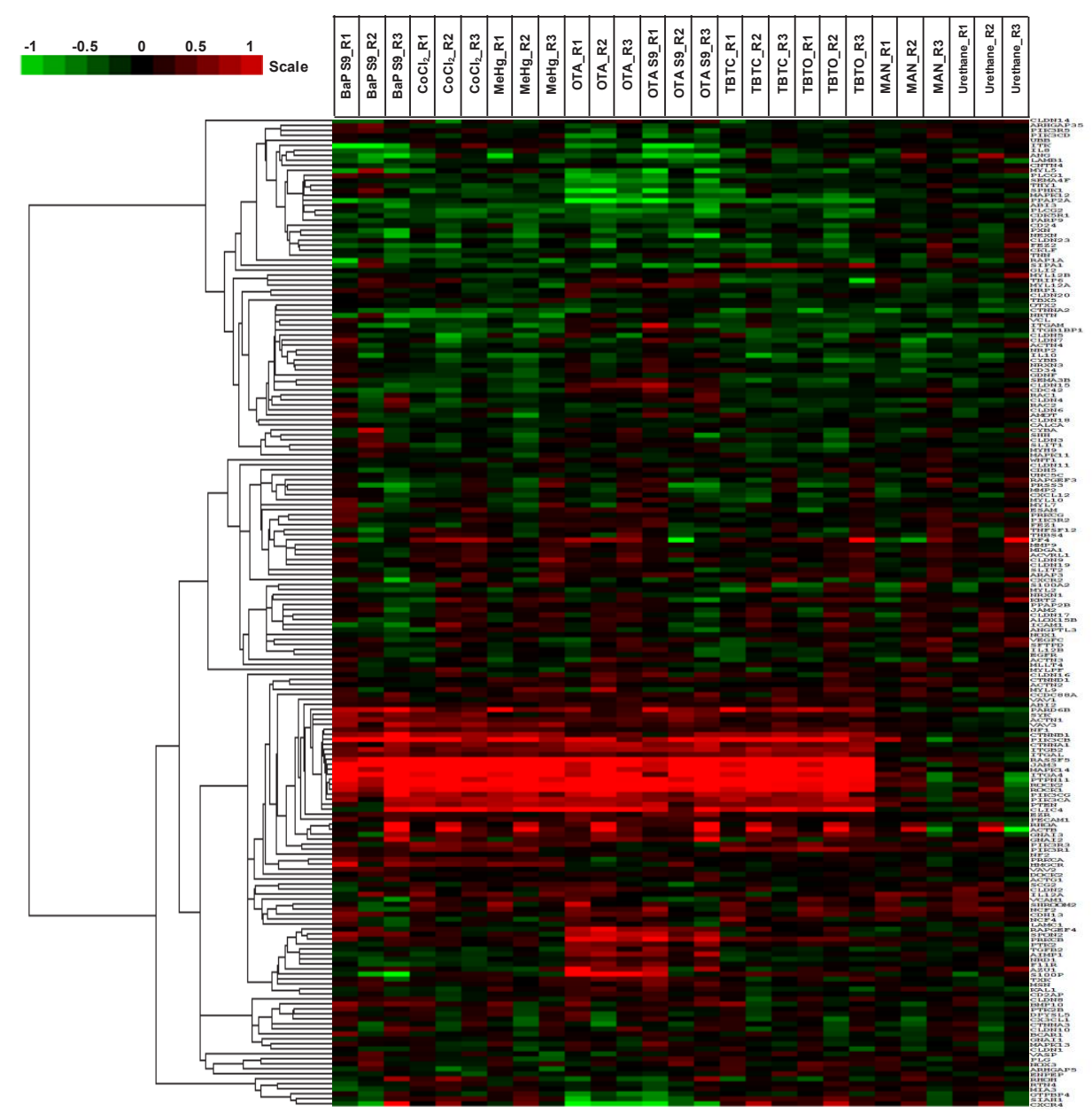

FIG. 1. Unsupervised hierarchical clustering of 198 genes that are known to be related with cell migration. Heat map visualizes the up- or down-regulation of 198 genes in Jurkat cells in response to $6 h$ exposure to subcytotoxic levels of seven immunotoxicants and two nonimmunotoxic control compounds. Green represents down regulation, black no regulation, and red up regulation. The colour intensity related to the 2 log ratio is indicated by the bar. For the abbreviations of all the compounds, see Table 2. 


\section{Modulation of CXCL12-mediated chemotaxis of Jurkat cells by immunotoxicants}

Based on GSEA analysis of the microarray data, seven immunotoxicants were identified as potential chemotaxis modulators (Table 2). Our previous results using the trans-well chemotaxis assay have shown that one of the seven immunotoxicants, TBTO, significantly inhibited the chemotaxis of Jurkat cells towards the chemokine CXCL12 (Shao et al., in preparation). Therefore, we continued to use the same assay set up to assess the effects of the other six immunotoxicants on CXCL12-mediated chemotaxis of Jurkat cells. Another set of six compounds were included in the experiment in order to verify whether compounds that were not indicated as chemotaxis modulators in the GSEA analysis would also be negative in the trans-well chemotaxis assay. These compounds include the immunotoxicants lindane and DON, the immunosuppressive drugs rapamycin and mycophenolic acid, and the nonimmunotoxic compounds urethane and mannitol. In order to avoid compounds that showing significant results because of a small standard deviation, a result between $90 \%$ and $110 \%$ was not considered as a biologically relevant effect. Using this criterion, our results indicated that six out of the 13 tested compounds, i.e., $\mathrm{CoCl}_{2}, \mathrm{MeHg}$, mycophenolic acid, ochratoxin A, S9-treated ochratoxin A, and TBTO, significantly inhibited the chemotaxis of Jurkat cells towards the chemokine CXCL12 (Figure 2). Ochratoxin A was shown to be the most potent chemotaxis inhibitor, as it decreased the percentage of migrated cells to $39.7 \%$. After S9 treatment, ochratoxin A still decreased the percentage of migrated cells to $71.1 \%$ (Figure 2). Mycophenolic acid decreased the percentage of migrated cells to $65 \%$ (Figure 2), although it was not identified as a chemotaxis modulator based on its effects on the mRNA expression of genes that are known to be related with cell migration (Table 2). The mTOR inhibitor rapamycin had no effect on the chemotaxis of Jurkat cell (Figure 2), which indicated that the mTOR pathway was not involved in CXCL12-mediated chemotaxis of Jurkat cells. Another compound that has also been shown to potently inhibit the mTOR signaling by our previous protein phosphorylation data (Shao et al., in preparation), lindane, did not affect the chemotaxis of Jurkat cells either (Figure 2), which also corroborates with the finding that the mTOR pathway is not crucial for inhibiting CXCL12-mediated chemotaxis of Jurkat cells. 


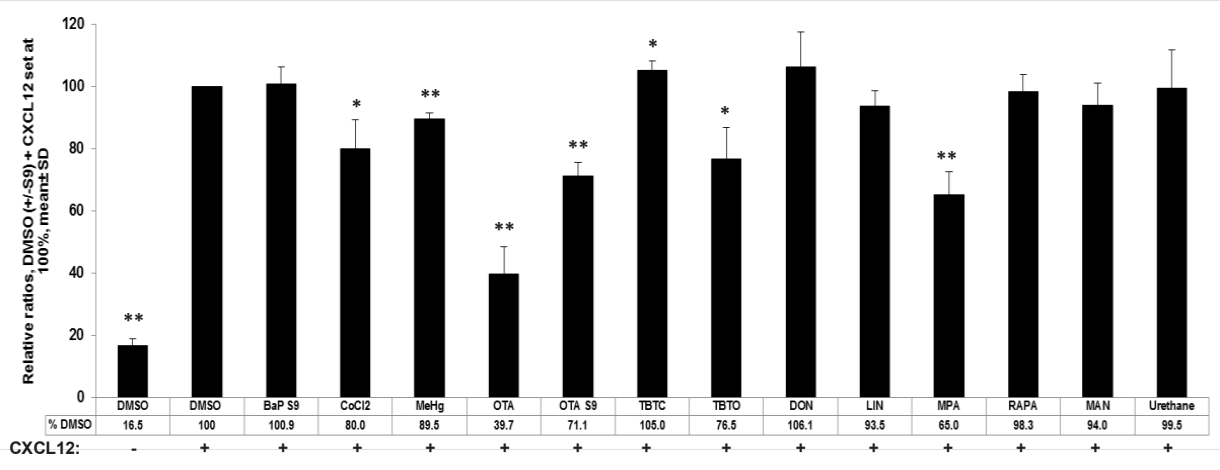

FIG. 2. Modulation of CXCL12-CXCR4 mediated chemotaxis of Jurkat cells by different immunotoxicants and immunosuppressive drugs. Jurkat cells were exposed to the test compounds for $24 \mathrm{~h}$. In the last $2 \mathrm{~h}$ of exposure, cells were counted and transferred to the apical compartments of the trans-well system with medium containing CXCL12 in the basolateral compartment. Migration was allowed for $2 \mathrm{~h}$. The percentages of migrated Jurkat cells were then calculated for each compound. Relative ratios were calculated by setting the average result of the carrier control, i.e., DMSO (+/-S9), at 100\%. Data presented in the figure are the average results ( \pm SD) of three independent experiments. *: $p$ value $<0.05$, **: $p$ value $<0.01$, two sample equal variance $T$ test, two tailed.

A.

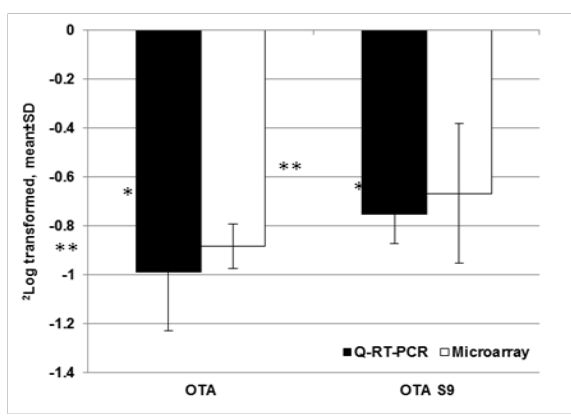

B.

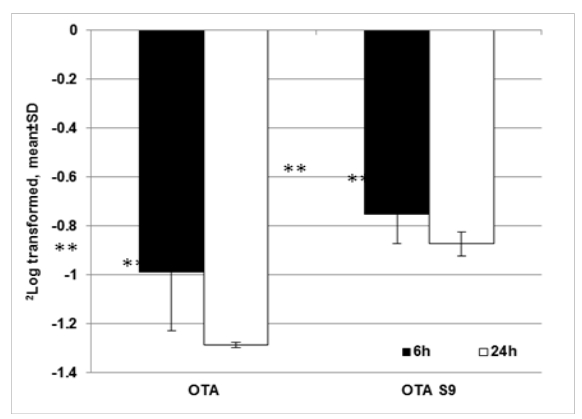

FIG 3. Quantitative Real-Time PCR verification of the expression levels of CXCR4 in Jurkat cell upon exposure to ochratoxin A (OTA) or S9-treated ochratoxin A (OTA S9). The bar graphs show the expression levels of CXCR4 in Jurkat cells in response to OTA or OTA S9 exposure: $(A)$ comparison between the expression levels of CXCR4 in microarray and qRT-PCR upon $6 \mathrm{~h}$ exposure to OTA or OTA S9; (B) time course expression levels of CXCR4 in qRT-PCR upon 6 or $24 \mathrm{~h}$ exposure to OTA or OTA S9. Values are 2 Log ratios of treatment compared to the average of DMSO controls. *: $p$ value $<0.05,{ }^{* *}: p$ value $<0.01$, two sample equal variance $T$ test, two tailed. 
Real time quantitative PCR (qRT-PCR) verification on the expression levels of CXCR4

Our transcriptome analysis determined that $6 \mathrm{~h}$ of exposure to either ochratoxin A or S9-treated ochratoxin A, but not to other compounds, decreased the expression levels of CXCR4, which encodes the receptor for chemokine CXCL12 (Figure 1). To verify these results, we performed GRT-PCR on CXCR4 in Jurkat cells exposed for 6 or $24 \mathrm{~h}$ to ochratoxin A or S9-treated ochraotoxin A. Both ochratoxin A and S9-treated ochratoxin A significantly downregulated CXCR4 after 6 and $24 \mathrm{~h}$ (Figure $3)$.

\section{Discussion}

Our previous studies on the effects of the model immunotoxicant TBTO on the transcriptome and protein phosphorylation profile of Jurkat cells indicated that TВTO affected the process of cell migration. This result was further confirmed by an in vitro trans-well chemotaxis assay using the chemokine CXCL12 (Shao et al., in preparation). The present study aimed to identify more immunotoxic compounds exhibiting chemotaxis-modulating properties. To this end, we pre-selected seven potential chemotaxis modulators based on their effects on gene expression profiling of Jurkat cells, and tested them in the trans-well chemotaxis assay. Another set of six compounds was tested in the chemotaxis assay as well, in order to check whether compounds that were not indicated as chemotaxis modulators by their effects on gene expression are negative in the trans-well chemotaxis assay. Our results showed that six out of the 13 compounds, including TBTO, significantly inhibited the chemotaxis of Jurkat cells towards the chemokine CXCL12. Furthermore, the transcriptome and protein phosphorylation profiles of the test compounds indicate that the mechanisms responsible for this inhibition are different for different immunotoxicants.

All seven compounds that have been pre-selected as potential chemotaxis modulators showed upregulation of the genes encoding leukocyte integrins (ITGAL, ITGA4, and ITGB2), Rho-associated, coiled-coil containing protein kinases (ROCK1 and ROCK2), and PI3Kinase subunits (PIK3CA, PIK3CB, and PIK3CG) (Figure 1). Integrins are adhesion receptors that play crucial roles in controlling lymphocytes to attach to and migrate through endothelium and interact with antigen-presenting cells (Kinashi et al., 2007). Both PI3K and RhoA/ROCK signaling have been demonstrated to be indispensable in the migration of lymphocytes (Ward, 2006; Worthylake and Burridge, 2003). Based on the gene expression data, it could be expected that these compounds increased the migration of Jurkat cells. However, in the trans-well chemotaxis assay, five out of seven compounds showed an inhibitory effect on the chemotaxis of Jurkat cells. Therefore, the observed upregulation of the above mentioned genes could be an adaptive response of the cells to restore their chemotactic capability.

The parent compound ochratoxin A and S9-treated ochratoxin A were identified as the most potent chemotaxis inhibitors, which reduced the percentage of migrated Jurkat cells to 39.7 and $71.1 \%$ after 24h exposure, respectively (Figure 2). The inhibitory effect of ochratoxin A was decreased by S9 treatment indicating that the metabolites of ochratoxin A had no or less effects on Jurkat cell 
chemotaxis compared to the parent compound. This observation agrees with the effects of ochratoxin $A$ and S9 treated ochratoxin A on the expression levels of CXCR4. Both treatments significantly downregulated CXCR4 mRNA expression levels in Jurkat cells, but ochratoxin A had a larger effect than S9-treated ochratoxin A (Figure 1 and 3 ). These results suggest that ochratoxin $A$ inhibits chemotaxis via downregulation of CXCR4. In addition, our transcriptomics results showed that ochratoxin A and S9-treated ochratoxin A downregulate the gene coding for the Tec family kinase ITK (Figure 1). ITK has been shown to regulate CXCR4-mediated chemotaxis of T cells, as ITK null T cells exhibited reduced chemotaxis towards CXCL12 (Fischer et al., 2004). Another gene that was downregulated by ochratoxin A, PLCG2, has been linked with leukocyte migration before: a patient carrying a missense mutation in PLCG2 had impaired leukocyte chemotaxis (Zhou et al., 2012). Our results are in concordance with those of Richetti et al., who demonstrated that several mycotoxins, including ochratoxin $\mathrm{B}$, are potent inhibitors of chemotactic peptide-induced chemotaxis of human primary neutrophils (Richetti et al., 2005).

The present study also identified the heavy metals methyl mercury and $\mathrm{CoCl}_{2}$ as chemotaxis inhibitors, which reduced the percentage of migrated Jurkat cells to 89.5 and $80.0 \%$ after $24 \mathrm{~h}$ exposure, respectively (Figure 2). Impairment of neutrophil chemotaxis has been reported as one of the immunotoxic effects of mercury before, as the migration and respiratory burst of neutrophils isolated from workers exposed to "safe levels" of mercury have been found to decrease significantly and the reduced chemotaxis persisted for 6 months after exposure ended (Perlingeiro and Queiroz, 1995). $\mathrm{CoCl}_{2}$ has been reported to indirectly stimulate chemotaxis of neutrophils and lymphocytes by promoting the secretion of chemoattractants such as IL8 and monocyte-chemoattractant protein 1 (MCP1) from endothelial cells (Kim et al., 2006; Ninomiya et al., 2013). However, a direct inhibiting effect of $\mathrm{CoCl}_{2}$ on the chemotaxis of lymphocytes has not been reported yet.

Among the seven compounds that showed significant effects on the expression of the genes belonging to the combined gene set for cell migration, S9-treated benzo[a]pyrene and TBTC did not affect CXCL12-mediated chemotaxis of Jurkat cells (Figure 2). This indicates that the changes at mRNA levels alone may not be sufficient to modulate CXCL12-mediated chemotaxis. The observation that TBTC had no effect on CXCL12-mediated chemotaxis of Jurkat cells was not expected, since it had similar effects on gene expression in Jurkat cells as compared to TBTO (Figure 1). However, we did observe a big difference between the effects of these two compounds on the protein phosphorylation profile of Jurkat cells, for instance TBTO inhibited the p70S6K pathway, and activated the kinases STAT3, ErbB3, Akt, EphB1 and EphB3, whereas, TBTC had no effects on these cellular signaling events (Shao et al., in preparation). Therefore, the different effects of TBTO and TBTC on CXCL12-mediated chemotaxis of Jurkat cells might be associated to their different effects on protein phosphorylation in Jurkat cells. This might also be the reason why benzo[a[pyrene had no effect on chemotaxis of Jurkat cells.

In order to check whether compounds that were not indicated as chemotaxis modulators in gene expression data analysis are also negative in the trans-well chemotaxis assay, we also included 
lindane, DON, rapamycin, mycophenolic acid, urethane, and mannitol in the in vitro trans-well chemotaxis assay. Among these six compounds, the immunosuppressive drug mycophenolic acid decreased the percentage of migrated Jurkat cells to $65.0 \%$ (Figure 2). Mycophenolic acid has been reported to inhibit the expression of the chemoattractant MCP-1 in epithelial cells (Shui et al., 2010; Schuchardt et al., 2011), which might indicate an indirect effect on the chemotaxis of immune cells. However, no studies have been reported focusing on the direct inhibiting effect of mycophenolic aicd on the chemotaxis of lymphocytes. In addition, this results again indicates that CXCL12-mediated chemotaxis is not only regulated at mRNA level.

It was expected that the mTOR inhibitor rapamycin would inhibit CXCL12-mediated chemotaxis of Jurkat cells, as mTOR has been shown to be involved in the migration of numerous cell types (Sakakibara et al., 2005; Liu and Parent, 2011; He et al., 2013), including CXCL12-mediated chemotaxis of human primary $T$ lymphocytes (Munk et al., 2011). However, rapamycin did not affect CXCL12-mediated chemotaxis of Jurkat cells in our experiments (Figure 2), which actually agrees with our results from GSEA analysis that rapamycin had no significant effect on the migration gene set (Table 2). In addition, lindane and DON, two other compounds that have been shown as modulators of the p70S6K pathway (Shao et al., in preparation), which is the pathway under direct control of mTOR, had no effect on CXCL12-mediated chemotaxis of Jurkat cells either. Therefore, it is evident that the mTOR pathway is not involved in the chemotaxis of Jurkat cells in response to CXCL12. Thus, in contrast to other cell types, inhibition of the mTOR pathway does not negatively affect the chemotaxis of Jurkat cells.

In summary, we have identified seven immunotoxicants as potential chemotaxis modulators based on their effects on the transcriptome of Jurkat cells. Five out of these seven compounds were confirmed as chemotaxis inhibitors in an in vitro trans-well chemotaxis assay using the chemokine CXCL12. Based on the transcriptome and protein phosphorylation data of the immunotoxicants, we propose that the mechanisms behind the inhibition of chemotaxis are different for different immunotoxicants. For instance, our finding that ochratoxin A and S9-treated ochratoxin A inhibited CXCL12 mediatedchemotaxis of Jurkat cells and downregulated CXCR4 mRNA expression suggests that the chemotaxis-inhibitory effects of ochratoxin A might be mediated via CXCR4 downregulation. However, the inhibitory effect of the organotin compound TBTO might be caused by its effects at both mRNA and phosphoprotein levels. Furthermore, our results indicated that, unlike human primary $T$ cells, the mTOR pathway is not involved in CXCL12-mediated chemotaxis of Jurkat cells. Thus, chemotaxis assays based on Jurkat cells could create false-negative results for those compounds that affect chemotaxis through mTOR signaling.

\section{Funding information}

This study was financially supported by the Netherlands Genomics Initiative, the Netherlands Toxicogenomics Centre (grant 05060510). 
Supplementary table 1. List of 198 genes that were used as migration gene set in GSEA analysis.

\begin{tabular}{|c|c|c|}
\hline Gene Symbol & Description & mRNA accession \\
\hline $\mathrm{ABI2}$ & abl-interactor 2 & NM_005759 \\
\hline $\mathrm{ABI} \mid 3$ & ABI family, member 3 & NM_001135186 \\
\hline ACTB & actin, beta & NM_001101 \\
\hline ACTG1 & actin, gamma 1 & NM_001199954 \\
\hline ACTN1 & actinin, alpha 1 & NM_001102 \\
\hline ACTN2 & actinin, alpha 2 & NM_001103 \\
\hline ACTN3 & actinin, alpha 3 & NM_001104 \\
\hline ACTN4 & actinin, alpha 4 & NM_004924 \\
\hline ACVRL1 & activin A receptor type II-like 1 & NM_000020 \\
\hline AIMP1 & $\begin{array}{l}\text { aminoacyl tRNA synthetase complex-interacting } \\
\text { multifunctional protein } 1\end{array}$ & NM_001142415 \\
\hline ALOX15B & arachidonate 15-lipoxygenase, type B & NM_001039130 \\
\hline AMOT & angiomotin & NM_001113490 \\
\hline ANG & angiogenin, ribonuclease, RNase $A$ family, 5 & NM_001097577 \\
\hline ANGPTL3 & angiopoietin-like 3 & NM_014495 \\
\hline ARAP3 & $\begin{array}{l}\text { ArfGAP with RhoGAP domain, ankyrin repeat and } \mathrm{PH} \text { domain } \\
3\end{array}$ & NM_022481 \\
\hline ARHGAP35 & Rho GTPase activating protein 35 & NM_004491 \\
\hline ARHGAP5 & Rho GTPase activating protein 5 & NM_001030055 \\
\hline AZU1 & azurocidin 1 & NM_001700 \\
\hline BCAR1 & breast cancer anti-estrogen resistance 1 & NM_001170714 \\
\hline BMP10 & bone morphogenetic protein 10 & NM_014482 \\
\hline CALCA & calcitonin-related polypeptide alpha & NM_001033952 \\
\hline CCDC88A & coiled-coil domain containing $88 \mathrm{~A}$ & NM_001135597 \\
\hline CD24 & CD24 molecule & NM_013230 \\
\hline CD2AP & CD2-associated protein & NM_012120 \\
\hline CD34 & CD34 molecule & NM_001025109 \\
\hline CDC42 & cell division cycle 42 (GTP binding protein, $25 \mathrm{kDa}$ ) & NM_001039802 \\
\hline $\mathrm{CDH} 13$ & cadherin $13, \mathrm{H}$-cadherin (heart) & NM_001220488 \\
\hline $\mathrm{CDH} 5$ & cadherin 5 , type 2 (vascular endothelium) & NM_001795 \\
\hline CDK5R1 & cyclin-dependent kinase 5 , regulatory subunit 1 (p35) & NM_003885 \\
\hline CKLF & chemokine-like factor & NM_001040138 \\
\hline
\end{tabular}




\begin{tabular}{|c|c|c|}
\hline CLDN1 & claudin 1 & NM_021101 \\
\hline CLDN10 & claudin 10 & NM_001160100 \\
\hline CLDN11 & claudin 11 & NM_001185056 \\
\hline CLDN14 & claudin 14 & NM_001146077 \\
\hline CLDN15 & claudin 15 & NM_001185080 \\
\hline CLDN16 & claudin 16 & NM_006580 \\
\hline CLDN17 & claudin 17 & NM_012131 \\
\hline CLDN18 & claudin 18 & NM_001002026 \\
\hline CLDN19 & claudin 19 & NM_001123395 \\
\hline CLDN2 & claudin 2 & NM_001171092 \\
\hline CLDN20 & claudin 20 & NM_001001346 \\
\hline CLDN23 & claudin 23 & NM_194284 \\
\hline CLDN3 & claudin 3 & NM_001306 \\
\hline CLDN4 & claudin 4 & NM_001305 \\
\hline CLDN5 & claudin 5 & NM_001130861 \\
\hline CLDN6 & claudin 6 & NM_021195 \\
\hline CLDN7 & claudin 7 & NM_001185022 \\
\hline CLDN8 & claudin 8 & NM_199328 \\
\hline CLDN9 & claudin 9 & NM_020982 \\
\hline CLIC4 & chloride intracellular channel 4 & NM_013943 \\
\hline CNTN4 & contactin 4 & NM_001206955 \\
\hline CTNNA1 & catenin (cadherin-associated protein), alpha 1, 102kDa & NM_001903 \\
\hline CTNNA2 & catenin (cadherin-associated protein), alpha 2 & NM_001164883 \\
\hline CTNNA3 & catenin (cadherin-associated protein), alpha 3 & NM 001127384 \\
\hline CTNNB1 & catenin (cadherin-associated protein), beta $1,88 \mathrm{kDa}$ & NM_001098209 \\
\hline CTNND1 & catenin (cadherin-associated protein), delta 1 & NM_001085458 \\
\hline CX3CL1 & chemokine (C-X3-C motif) ligand 1 & NM_002996 \\
\hline CXCL12 & chemokine (C-X-C motif) ligand 12 & NM_000609 \\
\hline CXCR2 & chemokine (C-X-C motif) receptor 2 & NM 001168298 \\
\hline CXCR4 & chemokine (C-X-C motif) receptor 4 & NM 001008540 \\
\hline CYBA & cytochrome b-245, alpha polypeptide & NM 000101 \\
\hline CYBB & cytochrome b-245, beta polypeptide & NM 000397 \\
\hline DOCK2 & dedicator of cytokinesis 2 & NM 004946 \\
\hline
\end{tabular}




\begin{tabular}{|c|c|}
\hline DPYSL5 & dihydropyrimidinase-like 5 \\
\hline EGFR & epidermal growth factor receptor \\
\hline ENPEP & glutamyl aminopeptidase (aminopeptidase A) \\
\hline ESAM & endothelial cell adhesion molecule \\
\hline EZR & ezrin \\
\hline F11R & F11 receptor \\
\hline FEZ1 & fasciculation and elongation protein zeta 1 (zygin I) \\
\hline FEZ2 & fasciculation and elongation protein zeta 2 (zygin II) \\
\hline GDNF & glial cell derived neurotrophic factor \\
\hline GLI2 & GLI family zinc finger 2 \\
\hline GNAl1 & $\begin{array}{c}\text { guanine nucleotide binding protein (G protein), alpha } \\
\text { inhibiting activity polypeptide } 1\end{array}$ \\
\hline GNAI2 & $\begin{array}{c}\text { guanine nucleotide binding protein ( } G \text { protein), alpha } \\
\text { inhibiting activity polypeptide } 2\end{array}$ \\
\hline GNAI3 & $\begin{array}{l}\text { guanine nucleotide binding protein ( } \mathrm{G} \text { protein), alpha } \\
\text { inhibiting activity polypeptide } 3\end{array}$ \\
\hline GTPBP4 & GTP binding protein 4 \\
\hline HMGCR & 3-hydroxy-3-methylglutaryl-CoA reductase \\
\hline ICAM1 & intercellular adhesion molecule 1 \\
\hline IL10 & interleukin 10 \\
\hline IL12A & $\begin{array}{l}\text { interleukin } 12 \mathrm{~A} \text { (natural killer cell stimulatory factor } 1 \text {, } \\
\text { cytotoxic lymphocyte maturation factor } 1, \mathrm{p} 35 \text { ) }\end{array}$ \\
\hline IL12B & $\begin{array}{l}\text { interleukin } 12 \mathrm{~B} \text { (natural killer cell stimulatory factor } 2 \text {, } \\
\text { cytotoxic lymphocyte maturation factor } 2, \mathrm{p} 40 \text { ) }\end{array}$ \\
\hline IL8 & interleukin 8 \\
\hline ITGA4 & $\begin{array}{c}\text { integrin, alpha } 4 \text { (antigen CD49D, alpha } 4 \text { subunit of VLA-4 } \\
\text { receptor) }\end{array}$ \\
\hline ITGAL & $\begin{array}{l}\text { integrin, alpha } L \text { (antigen CD11A ( } \mathrm{p} 180 \text { ), lymphocyte } \\
\text { function-associated antigen 1; alpha polypeptide) }\end{array}$ \\
\hline ITGAM & $\begin{array}{l}\text { integrin, alpha M (complement component } 3 \text { receptor } 3 \\
\text { subunit) }\end{array}$ \\
\hline ITGB1BP1 & integrin beta 1 binding protein 1 \\
\hline ITGB2 & $\begin{array}{c}\text { integrin, beta } 2 \text { (complement component } 3 \text { receptor } 3 \text { and } 4 \\
\text { subunit) }\end{array}$ \\
\hline ITK & IL2-inducible T-cell kinase \\
\hline JAM2 & junctional adhesion molecule 2 \\
\hline JAM3 & junctional adhesion molecule 3 \\
\hline KAL1 & Kallmann syndrome 1 sequence \\
\hline
\end{tabular}

NM_020134

NM_005228

NM_001977

NM_138961

NM_001111077

NM_016946

NM_005103

NM_001042548

NM_000514

NM_005270

NM_002069

NM_001166425

NM_006496

NM_012341

NM_000859

NM_000201

NM_000572

NM_000882

NM_002187

NM_000584

NM_000885

NM_001114380

NM_000632

NM_004763

NM_000211

NM_005546

NM_021219

NM_001205329

NM_000216 


\begin{tabular}{|c|c|c|}
\hline KRT2 & keratin 2 & NM_000423 \\
\hline LAMB1 & laminin, beta 1 & NM_002291 \\
\hline LAMC1 & laminin, gamma 1 (formerly LAMB2) & NM_002293 \\
\hline MAPK11 & mitogen-activated protein kinase 11 & NM_002751 \\
\hline MAPK12 & mitogen-activated protein kinase 12 & NM_002969 \\
\hline MAPK13 & mitogen-activated protein kinase 13 & NM_002754 \\
\hline MAPK14 & mitogen-activated protein kinase 14 & NM_001315 \\
\hline MDGA1 & MAM domain containing glycosylphosphatidylinositol anchor & NM_153487 \\
\hline MIA3 & melanoma inhibitory activity family, member 3 & NM_198551 \\
\hline MLLT4 & $\begin{array}{l}\text { myeloid/lymphoid or mixed-lineage leukemia (trithorax } \\
\text { homolog, Drosophila); translocated to, } 4\end{array}$ & NM_001040000 \\
\hline MMP2 & $\begin{array}{c}\text { matrix metallopeptidase } 2 \text { (gelatinase } \mathrm{A}, 72 \mathrm{kDa} \text { gelatinase, } \\
72 \mathrm{kDa} \text { type IV collagenase) }\end{array}$ & NM_001127891 \\
\hline MMP9 & $\begin{array}{l}\text { matrix metallopeptidase } 9 \text { (gelatinase } \mathrm{B}, 92 \mathrm{kDa} \text { gelatinase, } \\
\text { 92kDa type IV collagenase) }\end{array}$ & NM_004994 \\
\hline MSN & moesin & NM_002444 \\
\hline MYH9 & myosin, heavy chain 9 , non-muscle & NM_002473 \\
\hline MYL10 & myosin, light chain 10 , regulatory & NM_138403 \\
\hline MYL12A & myosin, light chain $12 \mathrm{~A}$, regulatory, non-sarcomeric & NM_006471 \\
\hline MYL12B & myosin, light chain $12 \mathrm{~B}$, regulatory & NM_001144944 \\
\hline MYL2 & myosin, light chain 2, regulatory, cardiac, slow & NM_000432 \\
\hline MYL5 & myosin, light chain 5 , regulatory & NM_002477 \\
\hline MYL7 & myosin, light chain 7 , regulatory & NM_021223 \\
\hline MYL9 & myosin, light chain 9 , regulatory & NM_006097 \\
\hline MYLPF & myosin light chain, phosphorylatable, fast skeletal muscle & NM_013292 \\
\hline NCF2 & neutrophil cytosolic factor 2 & NM_000433 \\
\hline NCF4 & neutrophil cytosolic factor $4,40 \mathrm{kDa}$ & NM_000631 \\
\hline NEXN & nexilin ( $\mathrm{F}$ actin binding protein) & NM_001172309 \\
\hline NF1 & neurofibromin 1 & NM_000267 \\
\hline NF2 & neurofibromin 2 (merlin) & NM_000268 \\
\hline NOX1 & NADPH oxidase 1 & NM_007052 \\
\hline NOX3 & NADPH oxidase 3 & NM_015718 \\
\hline NRD1 & nardilysin ( $\mathrm{N}$-arginine dibasic convertase) & NM_001101662 \\
\hline NRP1 & neuropilin 1 & NM_001024628 \\
\hline
\end{tabular}




\begin{tabular}{|c|c|c|}
\hline NRP2 & neuropilin 2 & NM_003872 \\
\hline NRTN & neurturin & NM_004558 \\
\hline NRXN1 & neurexin 1 & NM_001135659 \\
\hline NRXN3 & neurexin 3 & NM_001105250 \\
\hline OTX2 & orthodenticle homeobox 2 & NM_021728 \\
\hline PARD6B & par-6 partitioning defective 6 homolog beta (C. elegans) & NM_032521 \\
\hline PARP9 & poly (ADP-ribose) polymerase family, member 9 & NM_001146102 \\
\hline PECAM1 & platelet/endothelial cell adhesion molecule & NM_000442 \\
\hline PF4 & platelet factor 4 & NM_002619 \\
\hline PIK3CA & phosphoinositide-3-kinase, catalytic, alpha polypeptide & NM_006218 \\
\hline PIK3CB & phosphoinositide-3-kinase, catalytic, beta polypeptide & NM_006219 \\
\hline PIK3CD & phosphoinositide-3-kinase, catalytic, delta polypeptide & NM_005026 \\
\hline PIK3CG & phosphoinositide-3-kinase, catalytic, gamma polypeptide & NM_002649 \\
\hline PIK3R1 & phosphoinositide-3-kinase, regulatory subunit 1 (alpha) & NM_001242466 \\
\hline PIK3R2 & phosphoinositide-3-kinase, regulatory subunit 2 (beta) & NM_005027 \\
\hline PIK3R3 & phosphoinositide-3-kinase, regulatory subunit 3 (gamma) & NM_001114172 \\
\hline PIK3R5 & phosphoinositide-3-kinase, regulatory subunit 5 & NM_001142633 \\
\hline PLCG1 & phospholipase C, gamma 1 & NM_002660 \\
\hline PLCG2 & phospholipase C, gamma 2 (phosphatidylinositol-specific) & NM_002661 \\
\hline PLG & plasminogen & NM_000301 \\
\hline PPAP2A & phosphatidic acid phosphatase type $2 \mathrm{~A}$ & NM_003711 \\
\hline PPAP2B & phosphatidic acid phosphatase type $2 B$ & NM_003713 \\
\hline PRKCA & protein kinase $\mathrm{C}$, alpha & NM_002737 \\
\hline PRKCB & protein kinase $\mathrm{C}$, beta & NM_002738 \\
\hline PRKCG & protein kinase $\mathrm{C}$, gamma & NM_002739 \\
\hline PRSS3 & protease, serine, 3 & NM_001197097 \\
\hline PTEN & phosphatase and tensin homolog & NM_000314 \\
\hline PTK2 & PTK2 protein tyrosine kinase 2 & NM_001199649 \\
\hline PTK2B & PTK2B protein tyrosine kinase 2 beta & NM_004103 \\
\hline PTPN11 & protein tyrosine phosphatase, non-receptor type 11 & NM_002834 \\
\hline PXN & paxillin & NM_001080855 \\
\hline RAC1 & $\begin{array}{l}\text { ras-related C3 botulinum toxin substrate } 1 \text { (rho family, small } \\
\text { GTP binding protein Rac1) }\end{array}$ & NM_006908 \\
\hline RAC2 & ras-related C 3 botulinum toxin substrate 2 (rho family, small & NM_002872 \\
\hline
\end{tabular}




\begin{tabular}{|c|c|}
\hline & GTP binding protein Rac2) \\
\hline RAP1A & RAP1A, member of RAS oncogene family \\
\hline RAPGEF3 & Rap guanine nucleotide exchange factor (GEF) 3 \\
\hline RAPGEF4 & Rap guanine nucleotide exchange factor (GEF) 4 \\
\hline RASSF5 & Ras association (RalGDS/AF-6) domain family member 5 \\
\hline RHOA & ras homolog gene family, member $\mathrm{A}$ \\
\hline $\mathrm{RHOH}$ & ras homolog gene family, member $\mathrm{H}$ \\
\hline ROCK1 & Rho-associated, coiled-coil containing protein kinase 1 \\
\hline ROCK2 & Rho-associated, coiled-coil containing protein kinase 2 \\
\hline RTN4 & reticulon 4 \\
\hline S100A2 & S100 calcium binding protein A2 \\
\hline S100P & S100 calcium binding protein $P$ \\
\hline SCG2 & secretogranin II \\
\hline SEMA3B & $\begin{array}{l}\text { sema domain, immunoglobulin domain (Ig), short basic } \\
\text { domain, secreted, (semaphorin) 3B }\end{array}$ \\
\hline SEMA4F & $\begin{array}{l}\text { sema domain, immunoglobulin domain (Ig), transmembrane } \\
\text { domain (TM) and short cytoplasmic domain, (semaphorin) 4F }\end{array}$ \\
\hline SFTPD & surfactant protein $\mathrm{D}$ \\
\hline $\mathrm{SHH}$ & sonic hedgehog \\
\hline SHROOM2 & shroom family member 2 \\
\hline $\mathrm{SIAH} 1$ & seven in absentia homolog 1 (Drosophila) \\
\hline SIPA1 & signal-induced proliferation-associated 1 \\
\hline SLIT1 & slit homolog 1 (Drosophila) \\
\hline SLIT2 & slit homolog 2 (Drosophila) \\
\hline SPHK1 & sphingosine kinase 1 \\
\hline SPON2 & spondin 2, extracellular matrix protein \\
\hline SYK & spleen tyrosine kinase \\
\hline TBX5 & T-box 5 \\
\hline TGFB2 & transforming growth factor, beta 2 \\
\hline THBS4 & thrombospondin 4 \\
\hline THY1 & Thy- 1 cell surface antigen \\
\hline TNFSF12 & tumor necrosis factor (ligand) superfamily, member 12 \\
\hline TNN & tenascin $\mathrm{N}$ \\
\hline TRIP6 & thyroid hormone receptor interactor 6 \\
\hline
\end{tabular}

NM_001010935

NM_001098531

NM_001100397

NM_182663

NM_001664

NM_004310

NM_005406

NM_004850

NM_007008

NM_005978

NM_005980

NM_003469

NM_001005914

NM_004263

NM_003019

NM_000193

NM_001649

NM_001006610

NM_006747

NM_003061

NM_004787

NM_001142601

NM_001128325

NM_001135052

NM_000192

NM_001135599

NM_003248

NM_006288

NM_003809

NM_022093

NM_003302 


\begin{tabular}{c|c|c} 
TXK & TXK tyrosine kinase & NM_003328 \\
UBB & ubiquitin B & NM_018955 \\
UNC5C & unc-5 homolog C (C. elegans) & NM_003728 \\
VASP & vasodilator-stimulated phosphoprotein & NM_003370 \\
VAV1 & vav 1 guanine nucleotide exchange factor & NM_005428 \\
VAV2 & vav 2 guanine nucleotide exchange factor & NM_001134398 \\
VAV3 & vav 3 guanine nucleotide exchange factor & NM_001079874 \\
VCAM1 & vascular cell adhesion molecule 1 & NM_001078 \\
VCL & vinculin & NM_003373 \\
VEGFC & vascular endothelial growth factor C & NM_005429 \\
WNT1 & wingless-type MMTV integration site family, member 1 & NM_005430
\end{tabular}




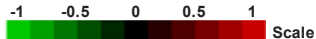

A.

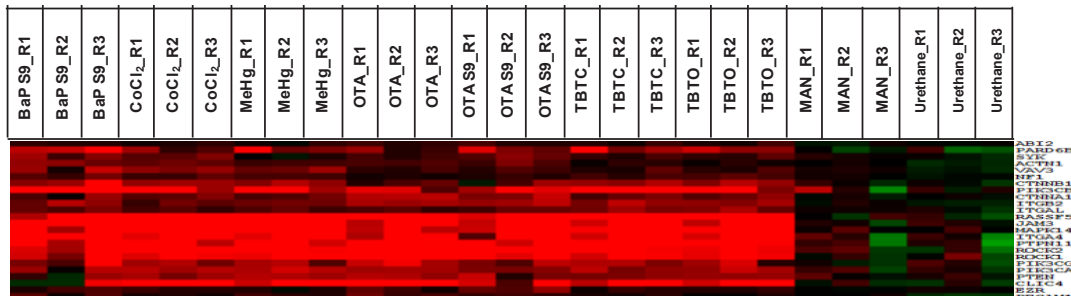

B.

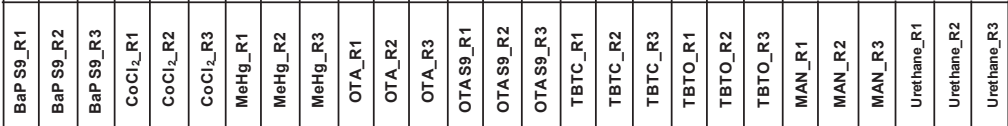

C.

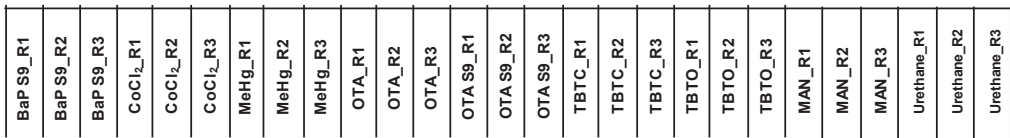

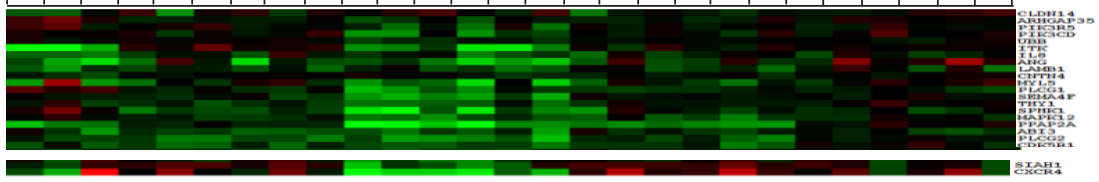

Supplementary fig. 1. Enlargements of Figure 1. Heat map visualizes the up-or downregulation of the genes in Jurkat cells in response to $6 \mathrm{~h}$ exposure to subcytotoxic levels of (A) seven immunotoxicants; (B) ochratoxin $A$ and S9-treated ochratoxin $A$; and (C) benzo[a]pyrene, ochratoxin $A$, and S9 treated-ochratoxin $A$. Green represents down regulation, black no regulation, and red up regulation. The colour intensity related to the 2log ratio is indicated by the bar. For the abbreviations of all the compounds, see Table 1. 


\section{Reference}

Balabanian, K., Lagane, B., Infantino, S., Chow, K. Y., Harriague, J., Moepps, B., ArenzanaSeisdedos, F., Thelen, M., and Bachelerie, F. (2005). The chemokine SDF-1/CXCL12 binds to and signals through the orphan receptor RDC1 in T lymphocytes. J. Biol. Chem. 280(42), 35760-35766.

Carlson, E. A., Li, Y., and Zelikoff, J. T. (2004). Benzo[a]pyrene-induced immunotoxicity in Japanese medaka (Oryzias latipes): relationship between lymphoid CYP1A activity and humoral immune suppression. Toxicol. Appl. Pharmacol. 201(1), 40-52.

Chen, H. (2005). Boyden chamber assay. Methods Mol. Biol. 294, 15-22.

Creppy, E. E., Størmer, F. C., Röschenthaler, R., and Dirheimer, G. (1983). Effects of two metabolites

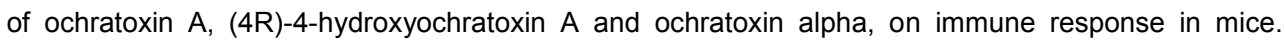
Infection and immunity 39(3), 1015-1018.

Del Prete, A., Shao, W. H., Mitola, S., Santoro, G., Sozzani, S., and Haribabu, B. (2007). Regulation of dendritic cell migration and adaptive immune response by leukotriene B4 receptors: a role for LTB4 in up-regulation of CCR7 expression and function. Blood 109(2), 626-631.

Ekhart, C., Rodenhuis, S., Beijnen, J., and Huitema, A. R. (2009). Carbamazepine induces bioactivation of cyclophosphamide and thiotepa. Cancer Chemother. Pharmacol. 63(3), 543-547.

Fischer, A. M., Mercer, J. C., lyer, A., Ragin, M. J., and August, A. (2004). Regulation of CXC Chemokine Receptor 4-mediated Migration by the Tec Family Tyrosine Kinase ITK. J. Biol. Chem. 279(28), 29816-29820.

Gerrits, H., van Ingen Schenau, D. S., Bakker, N. E., van Disseldorp, A. J., Strik, A., Hermens, L. S., Koenen, T. B., Krajnc-Franken, M. A., and Gossen, J. A. (2008). Early postnatal lethality and cardiovascular defects in CXCR7-deficient mice. Genesis 46(5), 235-245.

He, Y., Li, D., Cook, S. L., Yoon, M.-S., Kapoor, A., Rao, C. V., Kenis, P. J. A., Chen, J., and Wang, F. (2013). Mammalian target of rapamycin and Rictor control neutrophil chemotaxis by regulating Rac/Cdc42 activity and the actin cytoskeleton. Mol. Biol. Cell 24(21), 3369-3380.

Kim, K. S., Rajagopal, V., Gonsalves, C., Johnson, C., and Kalra, V. K. (2006). A novel role of hypoxia-inducible factor in cobalt chloride- and hypoxia-mediated expression of IL-8 chemokine in human endothelial cells. J. Immunol. 177(10), 7211-7224.

Kinashi, T., (2007). Integrin regulation of lymphocyte trafficking: lessons from structural and signaling studies. Advances in Immunology 93, 185-227.

Kumar, A., Humphreys, T. D., Kremer, K. N., Bramati, P. S., Bradfield, L., Edgar, C. E., and Hedin, K. E. (2006). CXCR4 Physically Associates with the T Cell Receptor to Signal in T Cells. Immunity 25(2), 213-224.

Levoye, A., Balabanian, K., Baleux, F., Bachelerie, F., and Lagane, B. (2009). CXCR7 heterodimerizes with CXCR4 and regulates CXCL12-mediated G protein signaling. Blood 113(24), 6085-6093.

Liu, L., and Parent, C. A. (2011). TOR kinase complexes and cell migration. J. Cell Biol. 194(6), 815824. 
Madri, J. A., and Graesser, D. (2000). Cell migration in the immune system: the evolving inter-related roles of adhesion molecules and proteinases. Developmental Immunology 7(2-4), 103-116.

Moser, B., Wolf, M., Walz, A., and Loetscher, P. (2004). Chemokines: multiple levels of leukocyte migration control. Trends in Immunology 25(2), 75-84.

Munk, R., Ghosh, P., Ghosh, M. C., Saito, T., Xu, M., Carter, A., Indig, F., Taub, D. D., and Longo, D. L. (2011). Involvement of mTOR in CXCL12 mediated T cell signaling and migration. PLOS ONE 6(9), e24667.

Nagasawa, T., Hirota, S., Tachibana, K., Takakura, N., Nishikawa, S. I., Kitamura, Y., Yoshida, N., Kikutani, H., and Kishimoto, T. (1996). Defects of B-cell lymphopoiesis and bone-marrow myelopoiesis in mice lacking the CXC chemokine PBSF/SDF-1. Nature 382(6592), 635-638.

Newton, K., and Dixit, V. M. (2012). Signaling in innate immunity and inflammation. Cold Spring Harbor Perspectives in Biology 4(3).

Ninomiya, J. T., Kuzma, S. A., Schnettler, T. J., Krolikowski, J. G., Struve, J. A., and Weihrauch, D. (2013). Metal ions activate vascular endothelial cells and increase lymphocyte chemotaxis and binding. J. Orthop. Res. 31(9), 1484-1491.

Okabe, S., Fukuda, S., and Broxmeyer, H. (2002). Src kinase, but not the Src kinase family member p56lck, mediates stromal cell-derived factor $1 \alpha / C X C L 12$-induced chemotaxis of a $T$ cell line. J. Hematother. Stem Cell Res. 11(6), 923-928.

Perlingeiro, R., and Queiroz, M. (1995). Measurement of the respiratory burst and chemotaxis in polymorphonuclear leukocytes from mercury-exposed workers. Hum. Exp. Toxicol. 14(3), 281-6.

Richetti, A., Cavallaro, A., Ainis, T., and Fimiani, V. (2005). Effect of mycotoxins on some activities of isolated human neutrophils. Immunopharmacol. Immunotoxicol. 27(3), 433-446.

Sakakibara, K., Liu, B., Hollenbeck, S., and Kent, K. C. (2005). Rapamycin inhibits fibronectin-induced migration of the human arterial smooth muscle line (E47) through the mammalian target of rapamycin. American Journal of Physiology - Heart and Circulatory Physiology 288(6), H2861-H2868.

Schuchardt, M., Tölle, M., Prüfer, J., Huber, S., Huang, T., Höhne, M., Zidek, W., and Van Der Giet, M. (2011). Mycophenolic acid diminishes monocyte chemoattractant protein-1 production in vascular smooth muscle cells via ROS inhibition. Journal of Hypertension 29, e475-e476.

Shao, J., Katika, M. R., Schmeits, P. C. J., Hendriksen, P. J. M., van Loveren, H., Peijnenburg, A. A. C. M., and Volger, O. L. (2013). Toxicogenomics-based identification of mechanisms for direct immunotoxicity. Toxicol. Sci. 135(2), 328-346.

Shao, J., Stout, I., Hendriksen, P. J., van Loveren, H., Peijnenburg, A. A., and Volger, O. L. (in preparation). Protein phosphorylation profiling identifies potential mechanisms for direct immunotoxicity.

Shui, H., Gao, P., Si, X., and Ding, G. (2010). Mycophenolic acid inhibits albumin-induced MCP-1 expression in renal tubular epithelial cells through the p38 MAPK pathway. Mol Biol Rep 37(4), 17491754.

Sierro, F., Biben, C., Martínez-Muñoz, L., Mellado, M., Ransohoff, R. M., Li, M., Woehl, B., Leung, H., Groom, J., Batten, M., Harvey, R. P., Martínez-A, C., Mackay, C. R., and Mackay, F. (2007). Disrupted 
cardiac development but normal hematopoiesis in mice deficient in the second CXCL12/SDF-1 receptor, CXCR7. Proceedings of the National Academy of Sciences 104(37), 14759-14764.

Ticchioni, M., Charvet, C., Noraz, N., Lamy, L., Steinberg, M., Bernard, A., and Deckert, M. (2002). Signaling through ZAP-70 is required for CXCL12-mediated T-cell transendothelial migration. Blood 99(9), 3111-3118.

Tilton, B., Ho, L., Oberlin, E., Loetscher, P., Baleux, F., Clark-Lewis, I., and Thelen, M. (2000). Signal transduction by CXC chemokine receptor 4: stromal cell-derived factor 1 stimulates prolonged protein kinase B and extracellular signal-regulated kinase 2 activation in T lymphocytes. The Journal of Experimental Medicine 192(3), 313-324.

Vicente-Manzanares, M., Cruz-Adalia, A., Martín-Cófreces, N. B., Cabrero, J. R., Dosil, M., AlvaradoSánchez, B., Bustelo, X. R., and Sánchez-Madrid, F. (2005). Control of lymphocyte shape and the chemotactic response by the GTP exchange factor Vav. Blood 105(8), 3026-3034.

Vila-Coro, A. J., Rodriguez-Frade, J. M., Martin de Ana, A., Moreno-Ortiz, M. C., Martinez-A, C., and Mellado, M. (1999). The chemokine SDF-1a triggers CXCR4 receptor dimerization and activates the JAK/STAT pathway. The FASEB Journal 13(13), 1699-1710.

Voermans, C., Anthony, E. C., Mul, E., van der Schoot, E., and Hordijk, P. (2001). SDF-1-induced actin polymerization and migration in human hematopoietic progenitor cells. Experimental Hematology 29(12), 1456-1464.

Ward, S. G. (2006). T lymphocytes on the move: chemokines, PI 3-kinase and beyond. Trends in Immunology 27(2), 80-87.

Wojcechowskyj, J. A., Lee, J. Y., Seeholzer, S. H., and Doms, R. W. (2011). Quantitative phosphoproteomics of CXCL12 (SDF-1) signaling. PLoS ONE 6(9), e24918.

Worthylake, R. A., and Burridge, K. (2003). RhoA and ROCK promote migration by limiting membrane protrusions. J. Biol. Chem. 278(15), 13578-13584.

Zhou, Q., Lee, G. S., Brady, J., Datta, S., Katan, M., Sheikh, A., Martins, Marta S., Bunney, Tom D., Santich, Brian H., Moir, S., Kuhns, Douglas B., Priel, Debra A. L., Ombrello, A., Stone, D., Ombrello, M. J., Khan, J., Milner, J. D., Kastner, Daniel L., and Aksentijevich, I. (2012). A Hypermorphic missense mutation in PLCG2, encoding phospholipase CY2, causes a dominantly inherited autoinflammatory disease with immunodeficiency. Am. J. Hum. Genet. 91(4), 713-720.

Zou, Y. R., Kottmann, A. H., Kuroda, M., Taniuchi, I., and Littman, D. R. (1998). Function of the chemokine receptor CXCR4 in haematopoiesis and in cerebellar development 393(6685), 595-599. 


\title{
CHAPTER 5
}

\section{Microarray analysis of human peripheral blood mononuclear cells (PBMCs) treated with tributyltin-oxide}

(TBTO)

\author{
Madhumohan R. Katika, Jia Shao, Peter J. M. Hendriksen, \\ Jan Bergervoet, Henk van Loveren, and Ad A. C. M. Peijnenburg \\ In preparation.
}




\section{Abstract}

Tributyltin-oxide (TBTO) is an organotin compound, known to modulate immune functions in various cell types. Previously, we studied the effects of TBTO on the transcriptome of the human T lymphocyte Jurkat cell line, which provided insight in its modes of action. In order to verify whether the processes affected by TBTO in the Jurkat cell line would also be affected in primary cells, the present study investigated the effect of TBTO on whole genome gene expression in human peripheral blood mononuclear cells (PBMCs) in vitro. To this end, PBMCs derived from four different donors were exposed to 0.5 and $1 \mu \mathrm{M}$ TBTO for 6 and $24 \mathrm{~h}$ and subjected to DNA microarray analysis. Analysis of the array data revealed that TBTO affects various biological processes. TBTO inhibits protein synthesis, induces ER and oxidative stress, NF-kB/TNF- $\alpha$, NFAT pathway, T cell activation and apoptosis. Furthermore, gene set enrichment analysis (GSEA) showed that TBTO affects genes that specifically are related to lymphocyte subtypes ( $T, B$ and NK cells), monocytes, dendritic cells and granulocytes. Comparative analysis of the PBMC microarray results with the results previously obtained with Jurkat cells revealed that most of the biological processes affected by TBTO in the Jurkat cell line are also affected in PBMCs. 


\section{Introduction}

The future of toxicity testing is considered to be based on the use of human in vitro models that substitute or complement traditional animal tests (Hartung et al., 2011 and NRC, 2013). Within this view, the identification of primary cellular targets of toxicants and unraveling the mode of actions or adverse outcome pathways are crucial goals (Hartung et al., 2011 and Meek et al., 2014). Previously, we have used the human T lymphocyte Jurkat cell line in two toxicogenomics studies with different exposure conditions to detect the mode of actions of tributyltin-oxide (TBTO). Microarray analysis indicated that TBTO induces cellular stress responses like ER and oxidative stress at all tested concentrations (Katika et al., 2011; Shao et al., 2013), and T cell activation and apoptosis at higher concentrations (Katika et al., 2011). These cellular processes have also been confirmed by immunocytological and biochemical experiments (Katika et al., 2012). An important issue is whether these toxic events are induced in humans in vivo as well. Since it is unethical to expose human volunteers to TBTO, we exposed human PBMCs in vitro to TBTO followed by microarray analysis. We then assessed whether the mode of actions previously identified in Jurkat cells are also induced in PBMCs, and whether PBMCs allow to detect mode of actions that were not identified using Jurkat cells.

In daily life, humans and animals are exposed to a variety of immunotoxic compounds of which tributyltin-oxide (TBTO) is one of the best studied ones. TBTO is a member of the organotin family which has been shown to induce immunosuppression in rodents (Snoeij et al., 1987; Antizar-Ladislao, 2008). TBTO has been widely used as a biocide in agriculture, as a catalyst and stabilizer in polyurethane plastic industries and as antifouling agent in paints. Due to its wide range of applications it has highly accumulated in the environment and food (Fent, 1996; Granmo et al., 2002; AntizarLadislao, 2008; Camps et al., 2011). Humans are primarily exposed to TBTO through consumption of contaminated food such as milk, meat and fish (Takahashi et al., 1999; Inadera, 2006).

TBTO is known to affect the reproductive system, neuroendocrine function, liver and the immune system. Particularly, TBTO affects T lymphocyte-mediated immune responses and causes depletion of cortical thymocytes in rodents leading to immunosuppression (Snoeij et al., 1988). Tributyltin (TBT) compounds also decrease the activity of cells of the innate immune system, such as NK cells, eosinophils and macrophages (Aluoch et al., 2007; Sroka et al., 2008; Whalen et al., 2010). TBTO inhibits cell proliferation and induces apoptosis in human $\mathrm{T}$ lymphocyte Jurkat cells and rat thymocytes (Grundler et al., 2001; Berg et al., 2003; Baken et al., 2007; Katika et al., 2011). Proteomics studies on the mouse thymoma cell line EL-4 revealed that TBTO affects proteins involved in cell proliferation, protein synthesis and protein trafficking (Osman et al., 2009). A recent phosphoproteomic analysis showed that TBTO modulates the activity of proteins involved in proliferation and energy sensing pathways (Osman et al., 2012). Previously, we reported on the effect of TBTO on gene expression in the human $T$ cell line Jurkat.

The main aim of the present study was to investigate whether the in vitro effects of TBTO on whole genome mRNA levels in the human Jurkat cell line (Katika et al., 2011; Shao et al., 2013) would also 
occur in human immune cells. To that end, PBMCs derived from four different individuals were exposed for 6 and $24 \mathrm{~h}$ to TBTO $(0.5$ and $1 \mu \mathrm{M})$ and subjected to DNA microarray analysis. Finally, the PBMC microarray data were compared with the previously published transcriptomics data obtained in Jurkat cells.

\section{Materials and methods}

Chemicals. Tributyltin tri-oxide (TBTO) was purchased from Sigma Aldrich (Zwijndrecht, The Netherlands). TBTO was $97 \%$ pure and were dissolved in $96 \%$ ethanol.

Isolation and culturing of PBMCs. Buffycoats from four different individuals were obtained from Sanquin (Amsterdam, The Netherlands). Peripheral blood mono nuclear cells (PBMCs) were isolated from these buffy coats using Lymphoprep kit according to the manufacturer's protocols (Lymphoprep, Wemmel, Belgium). After isolation, PBMCs were washed with phosphate buffer saline (PBS) and cells were counted with a coulter counter (Beckman Coulter, Woerden, The Netherlands). PBMCs were cultured at $37{ }^{\circ} \mathrm{C}$ in a humidified atmosphere consisting of $5 \% \mathrm{CO}_{2}$ in RPMI-1640 medium supplemented with $10 \%(\mathrm{v} / \mathrm{v})$ heat-inactivated FBS, $2 \mathrm{mM}$ L-glutamine, $100 \mathrm{U} / \mathrm{ml}$ penicillin and $100 \mu \mathrm{g} / \mathrm{ml}$ streptomycin.

Cell viability. The WST-1 assay was used to determine the cytotoxic concentrations of TBTO (Roche diagnostics, Germany). The assay is based on the ability of viable cells to metabolize and convert the water soluble tetrazolium salt (WST-1) to a yellow colored formazan by mitochondrial dehydrogenases. PBMCs were seeded in a 96-well plate at a concentration of 500,000 cells $/ \mathrm{ml}$ in quadruplicates per condition. TBTO solutions or medium (vehicle controls) were added to a total volume of $100 \mu \mathrm{l}$. PBMCs were exposed for 6 and $24 \mathrm{~h}$ to TBTO $(0.25,0.5$ and $1 \mu \mathrm{M})$ or vehicle control (ethanol). During the last $2 \mathrm{~h}$ of exposure, $10 \mu \mathrm{l}$ of WST-1 reagent was added. Absorbance was measured at $450 \mathrm{~nm}$ in a calorimetric microplate reader (BioTek, Winooski, VT). TBTO stock solutions were diluted in RPMI-1640 medium without FCS. The final ethanol concentration in the medium controls was $<0.1 \%$ which was not exerting any effect on cell viability. Relative viability was measured by comparing the mean optical density of the TBTO treated samples with the mean optical density of the vehicle controls.

Gene expression profiling experiment. On the basis of the results of the cell viability study, suitable doses were selected for microarray experiments. PBMCs were cultured in 6-well plates overnight and exposed for 6 and $24 \mathrm{~h}$ to final doses of 0.5 and $1 \mu \mathrm{M}$ of TBTO. Exposures were performed in quadruplicate. 
Total RNA isolation. After exposure of PBMCs to TBTO for 6 and $24 \mathrm{~h}$, cells were harvested, centrifuged (10 min, $1200 \mathrm{prm}, 4^{\circ} \mathrm{C}$ ) and washed with phosphate buffered saline (PBS). The cell pellet was dissolved in Trizol reagent (Qiagen, Venlo, The Netherlands) and immediately stored at $-80^{\circ} \mathrm{C}$. Total RNA was isolated using the Qiagen RNAeasy kit following manufacturer's instructions. RNA concentration and purity were assessed using a NanoDrop spectrophotometer (NanoDrop 2000, Thermoscientific, via Isogen-lifescience, De Meern, The Netherlands) by measuring absorption ratios at $260 / 280$ and $230 / 280 \mathrm{~nm}$. The integrity of the RNA samples was examined using Bio-Rad's Experion system (Veenendaal, The Netherlands).

Microarray hybridization. Gene expression profiling was performed using Affymetrix U133 Plus 2.0 Arrays (Affymetrix, Santa Clara, CA). For each RNA sample, complementary cDNA was synthesized by using $5 \mu \mathrm{g}$ of total RNA and subsequent biotin-labeled cRNA was synthesized using GeneChip One-Cycle Target Labeling and control reagents including the One-Cycle cDNA synthesis kit, Poly-A RNA control kit, sample cleanup module, IVT labeling kit, and hybridization control kit (Affymetrix, Santa Clara, CA) according to the manufacturer's protocol. The yield of cRNA was measured spectrophotometrically (Nanodrop) and the integrity was determined using the bio analyzer before and after fragmentation of $15 \mu \mathrm{g}$ of the labeled cRNA. Fragmented samples were hybridized to the arrays for $16 \mathrm{~h}$ at $45^{\circ} \mathrm{C}$ (GeneChip Hybridization Oven 640; Affymetrix), after which the chips were washed and stained using the GeneChip hybridization, wash and stain kit using the GeneChip Fluidics Station 450 (Affymetrix, Santa Clara, CA). Chips were then scanned using the Affymetrix GeneChip Scanner 3000 7G, and raw data were extracted using the GeneChip Operating Software (Affymetrix, Santa Clara, CA). The scaling factor, background, percentage present spots, and housekeeping controls were checked for each chip.

Processing of microarray data. Quality control of the gene expression data was performed using Bioconductor packages (including simpleaffy and affyplm) through the R pipeline. GenePattern (Reich et al., 2006) was used for preprocessing of these data. The scaling factor, background, percentage present spots, and housekeeping controls were checked for each chip. Arrays were normalized using Robust Multichip Average (RMA), employing the Expression File Creator module. RMA is a common algorithm for converting raw Affymetrix data to gene expression values using MBNI Custom CDF, which contains updated probe set definitions for Entrez Gene IDs (http://brainarray.mbni.med.umich.edu/Brainarray/Database/CustomCDF/11.0.1/entrezg.asp). After normalization, $2 \log$ ratios were calculated for each array vs. the average expression of the control treatments at the same exposure time.

Hierarchial clustering and pathway analysis. Hierarchical clustering was performed using the programs Cluster (uncentered correlation; average linkage clustering) and Treeview (Eisen et al., 1998). For 
genes within the subclusters of the hierarchical cluster heat-map, pathway analysis was done using Metacore. This is an online program used for functional and biological interpretation of gene expression data. Metacore uses hypergeometric distribution to assess significances for overrepresentation of affected genes from signalling and metabolic pathways (Ekins et al., 2006). Pathways with a $p$ value $<10^{-5}$ were considered as significant.

Gene set enrichment analysis. For biological interpretation, data were also subjected to gene set enrichment analysis (GSEA). GSEA is a statistical analysis tool for microarray data that was used to detect biological processes affected and to provide insight into the affected molecular mechanisms. GSEA makes use of predefined gene sets that are based on previous experimental results and literature. GSEA has the advantage over other statistical tools that no initial filtering is applied to the dataset to select significantly differentially expressed genes. GSEA ranks all the genes on their expression ratios and determines whether a particular gene set is significantly enriched at the top or the bottom of the ranked list (Subramanian et al., 2005). This enables detection of significantly affected gene sets, while the fold change of expression of individual genes can be relatively modest. GSEA was performed for all treatment groups vs. controls at the same time point. Gene sets with a $p$ value $<0.05$ and an FDR value $<0.25$ were considered being significantly affected.

For GSEA analysis, we used gene sets from various sources,

1. Lymphocyte database: gene sets were taken from a database published by Shaffer et al., 2001 (Shaffer et al., 2001).

2. Tox-action (self-made).

3. Biocarta-2: gene sets were downloaded from biocarta database.

(http://www.biocarta.com/).

4. Transcription factor targets: gene sets were selected from microarray studies on a) toxic action of compounds or b) target genes of toxicity-related transcription factors.

5. Gene Ontology: gene sets, including those related to molecular function, biological process and cellular components, were downloaded from the Gene Ontology consortium (http://www.geneontology.org/).

6. Specific blood cell types: gene sets were selected from microarray studies on different leukocyte subsets (Hoffmann et al., 2003; Lyons et al., 2007).

Comparative analysis of microarray data. To evaluate whether processes and pathways affected by TBTO in human PBMCs are also affected by TBTO in Jurkat cells, the PBMC array data were 
compared with previously published microarray data on Jurkat cells treated with TBTO for 3, 6, 12 and $24 \mathrm{~h}$ (Katika et al., 2011). The microarray data were converted into 2 log ratios of treatment vs. average of the controls. The expression of the genes of several major pathways/processes including (e.g. ER stress, oxidative stress, NF-kB pathway, $T$ cell activation and apoptosis) was assessed for each of these microarray studies. Genes were filtered on $\geq 1.5$-fold up- or downregulation in at least three of the treatments. Those genes were hierarchically clustered and visualized in heat maps.

\section{Results}

Cell viability

Prior to the microarray experiments, cell viability was determined by the extent of WST-1 cleavage by mitochondrial dehydrogenases in TBTO exposed cells compared to the controls. Fig.1 shows the viability of PBMCs after 3,6 and $24 \mathrm{~h}$ of exposure to TBTO $(0.25,0.5,1$ and $2 \mu \mathrm{M})$. The viability of the PBMCs was seriously affected ( $<80 \%$ viability) already at $3 \mathrm{~h}$ by $2 \mu \mathrm{M}$ TBTO and at $24 \mathrm{~h}$ by 1 and 2 $\mu \mathrm{M}$ TBTO. In contrast, the lower doses TBTO $(0.25 \mu \mathrm{M})$ tended to give an increase of the WST-1 signal at $6 \mathrm{~h}$. Exposure of PBMCs to 0.5 and $1 \mu \mathrm{M}$ TBTO for $24 \mathrm{~h}$ reduced the viability to 80 and $40 \%$ respectively. Thus, except for the $24 \mathrm{~h} 1 \mu \mathrm{M}$ TBTO treatment, all conditions examined by microarray analysis were being considered noncytotoxic.

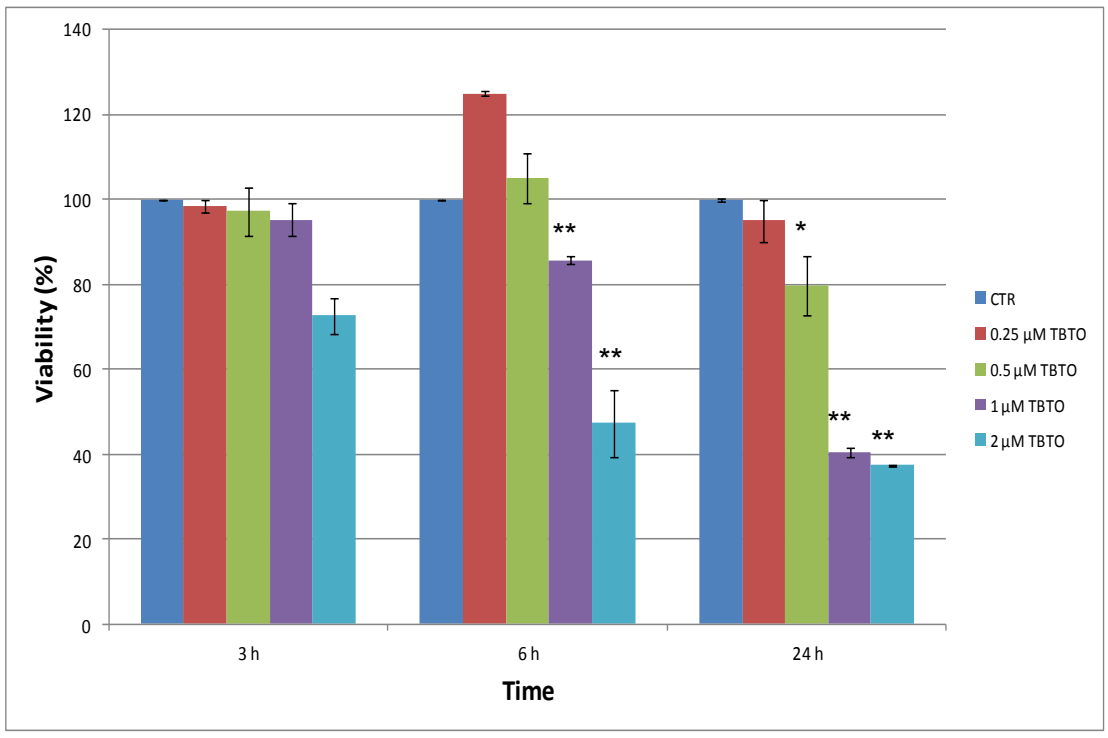

FIG. 1. Effect of TBTO on viability of PBMCs. Cell viability was determined using the WST-1 assay upon exposure of PBMCs for 6 and $24 h$ to $0.25,0.5,1$ and $2 \mu M$ TBTO. The viability after treatment is presented as percentage compared to the vehicle control at the same time point. Results shown are mean $\pm S D$ from quadruplicate exposures. **: $P<0.01$ (Student's T-test). 
Hierarchical clustering and pathway analysis

PBMCs exposed to 0.5 and $1 \mu \mathrm{M}$ TBTO for 6 and $24 \mathrm{~h}$ and RNA samples were subjected to microarray analysis. Then, unsupervised hierarchical clustering was performed for 4000 spots that were $\geq 2.5$ fold up- or down-regulated versus the average of the controls at the same time point in $\geq 3$ of 16 arrays and four main gene clusters, A-D, could be distinguished (Fig. 2). For genes within each of these clusters, pathway analysis was performed using Metacore. The majority of genes in cluster $A$ were downregulated by all conditions. Genes from cluster A were involved in immune response, NK cell signaling, cytokine signaling (IL5 and IL-17), chemotaxis in leukocytes, antigen presentation, PIP3 signaling in B cells and protein folding/membrane trafficking. Cluster B also contains genes that were mainly downregulated by all conditions of which $6 \mathrm{~h}$ exposure to $1 \mu \mathrm{M}$ TBTO induced the highest effect. Genes from cluster B were involved in ICOS (inducible co stimulator) pathway in T helper cells, TREM1 (triggering receptor expressed on myeloid cells 1) signaling, IL8 signaling in neutrophils and "Regulation of T cell function by CTLA4 and CD40 signaling. Genes in cluster C were upregulated by all conditions except for 6 h $1 \mu \mathrm{M}$ TBTO. Genes in cluster $C$ were involved in granzyme A \& B signaling pathways, NF-kB pathway, transcription regulation of granulocytes and PGE2 (prostaglandin E2) signaling. The main part of the genes of cluster $D$ was upregulated by all conditions. Cluster $D$ contains genes involved in oxidative stress, oncostatin M signaling, cytokine signaling (IL1, IL2, IL6, IL22 and IL3), ER stress, calcium signaling, apoptosis, NFAT signaling and T cell differentiation. 


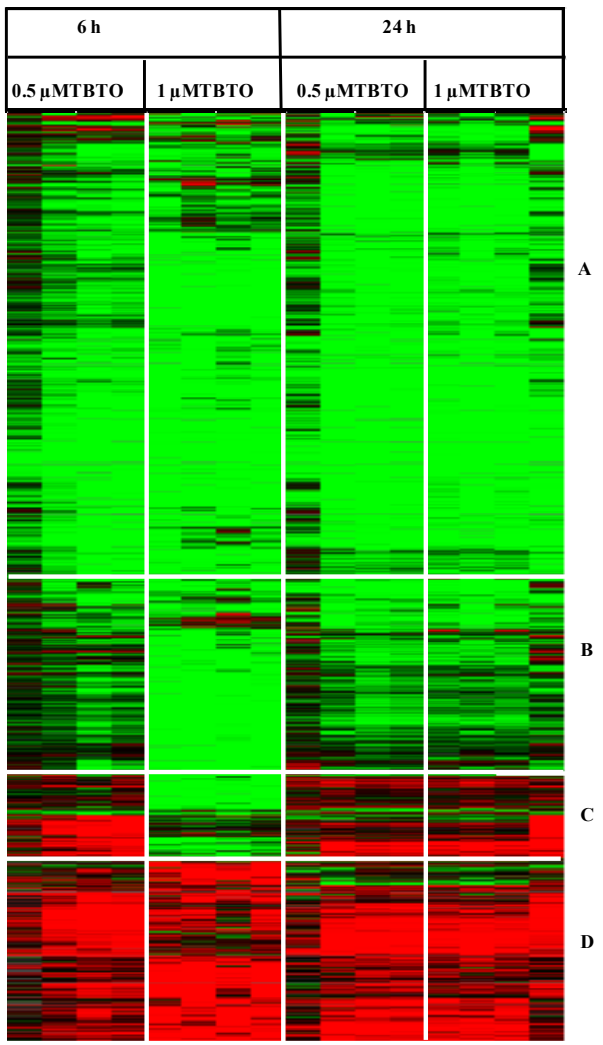

$\begin{array}{ccccccc}-0.8 & -0.4 & -0.2 & 0 & 0.2 & 0.4 & 0.8\end{array}$

Immune response, CD16 signaling in NK cells, IL5, IL-17 signaling, Leukocyte chemo taxis, antigen presentation by MHC-II, PIP3 signaling in B lymphocytes, protein folding/membrane trafficking,

Monocyte function and dendritic cell maturation and function.

B ICOS pathway in T helper cells, TREM1 signaling, IL8 signaling neutrophils, Regulation of $\mathrm{T}$ cell function by CTLA4, CD40 signaling.

Granzyme A \& B signaling, NF-kB signaling, Transcription regulation of granulocytes, PGE2 signaling.

Oxidative stress, Oncostatin M signaling, IL1, IL2, IL6, IL22,

IL3 signaling, ER stress, Ca+2 signaling, NFAT immune response, apoptosis, $\mathrm{T}$ cell differentiation

FIG. 2. Heat-map of genes differentially expressed upon exposure of PBMCs to TBTO. Differentially regulated genes were subjected to unsupervised hierarchical clustering analysis. For this analysis genes were selected that were $\geq 2$ times up- or downregulated versus the average of the control at the same exposure time in at least 3 of 16 arrays. Green represents downregulation, black no regulation, red upregulation. Four groups of gene expression patterns (clusters $A-D$ ) can be distinguished. Pathways or biological processes for which genes are overrepresented in a cluster according to Metacore analysis are indicated.

\section{Gene set enrichment analysis}

Gene set enrichment analysis (GSEA) was applied for the identification of biological processes / pathways affected by TBTO at the gene set level. GSEA was performed on the array data of the 6 and $24 \mathrm{~h}$ treatment groups and compared to those of the control samples at the same time point. Gene sets were taken from seven different collections (see Materials and Methods section). Up- and downregulation of genes within significantly affected gene sets were visualized in heat maps enabling comparison between the treatment groups. The results of GSEA analysis are described into more detail below. The following processes were found to be affected by TBTO (Fig. 3):

\section{RNA biosynthesis and translation}


TBTO upregulates genes related to RNA biosynthesis (Fig.3 and Supplementary fig. 1a). The gene set containing genes involved in translation was downregulated by $1 \mu \mathrm{M}$ TBTO at $24 \mathrm{~h}$ (Fig. 3 and Fig. 4A).

ER stress and unfolded protein response

TBTO upregulated the expression of genes involved in ER stress and unfolded protein response. ER stress related genes upregulated by TBTO included ATF3, DDIT3, EGR1 and XBP1 (Fig. 4B). Genes related to unfolded protein response (e.g., PFDN2, PFDN4, ERO1LB, СCT2, СCT3, CCT4, and CCT8) and members of DNAJ family heat shock proteins (e.g. DNAJA1, DNAJA4, DNAJB1, DNAJB2, DNAJB4, DNAJB9 and DNAJC3) were also upregulated by TBTO (Supplementary fig. 1b).

\section{$T$ cell activation}

As shown in Fig. $4 \mathrm{C}$ genes that are upregulated in $\mathrm{T}$ lymphocytes during $\mathrm{T}$ cell activation are also upregulated by TBTO. Key genes related to T cell activation (e.g. CD69, PMAIP1, FOSB, CTLA4, TAGAP, GEM, JUN, and NF-kB) are upregulated by TBTO. Genes were most upregulated by $0.5 \mu \mathrm{M}$ TBTO at $6 \mathrm{~h}$. The gene set containing genes involved in the NF-kB pathway (e.g. TNF, RELB, LTA, NF-kBI2, NF-kB2, IL6 and JUND) was upregulated by TBTO as well (Supplementary fig. 1c).

\section{Oxidative stress}

TBTO upregulates genes involved in oxidative stress and anti-oxidative response NRF2 pathway (Fig. 4D). Generally, genes were more upregulated at $24 \mathrm{~h}$ than at $6 \mathrm{~h}$.

\section{Mitochondria}

Genes related to mitochondrial function and mitochondrial ribosomal proteins were clearly upregulated by TBTO after 6 and particularly after $24 \mathrm{~h}$ (Fig. 3 and Supplementary fig. 1d).

\section{Apoptosis}

TBTO clearly induced upregulation of apoptosis related genes, including DEDD2, TNFSF14, SOD1, CD28, NPM1, TNF, PMAIP1, BRAF, BBC3, BAG3, CYS and GADD45A (Fig. 4E).

\section{Effect of TBTO on leukocyte type specific gene expression}

GSEA revealed that genes that are related to lymphocyte sub populations ( $T$, B and NK cells), monocytes, dendritic cells and granulocytes were downregulated by TBTO exposure (Fig. 3). TBTO downregulates the majority of genes that are related to T cells (CD4+ and CD8+), B cells, NK cells, granulocytes, monocytes and dendritic cells, particularly upon $24 \mathrm{~h}$ exposure (Supplementary fig. 2a$\mathrm{g}$, bottom part of heatmaps). 


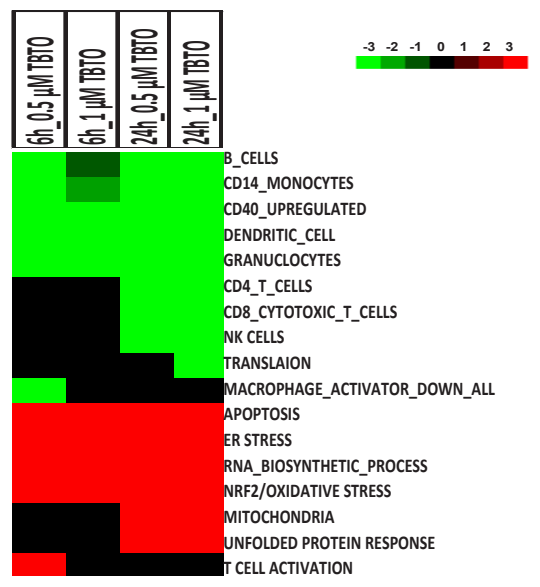

FIG. 3. Heatmap of processes significantly altered in PBMCs showing the overview of the effects of TBTO on a selection of gene sets that were run in GSEA (selection: $p$ value $\leq 0.05$, FDR $\leq 0.25$ ). Each line represents one gene set. Scale indicates statistical significance ( $p$-value) of the gene set. Green represents downregulation, red upregulation and black no effect.

A.

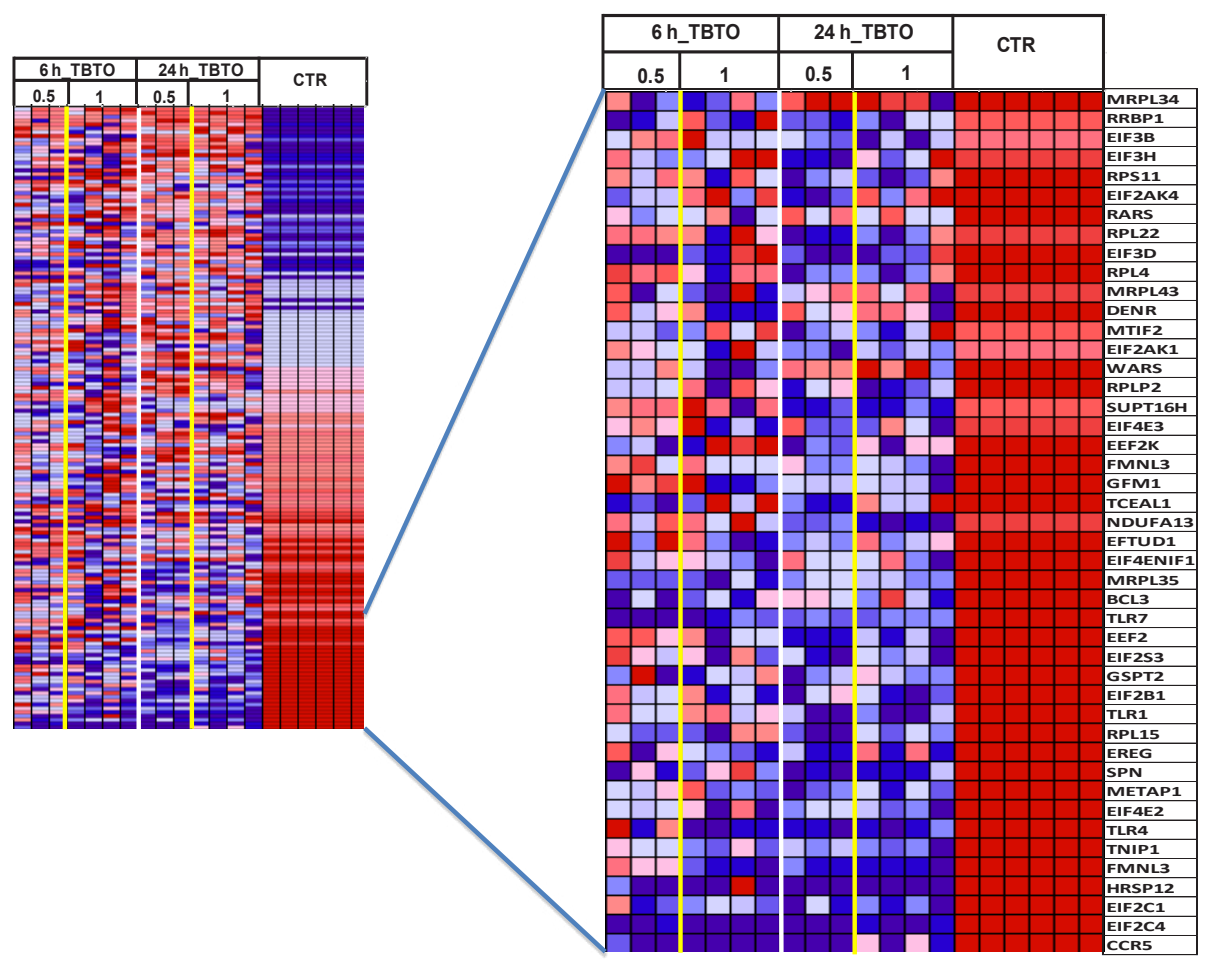


B.

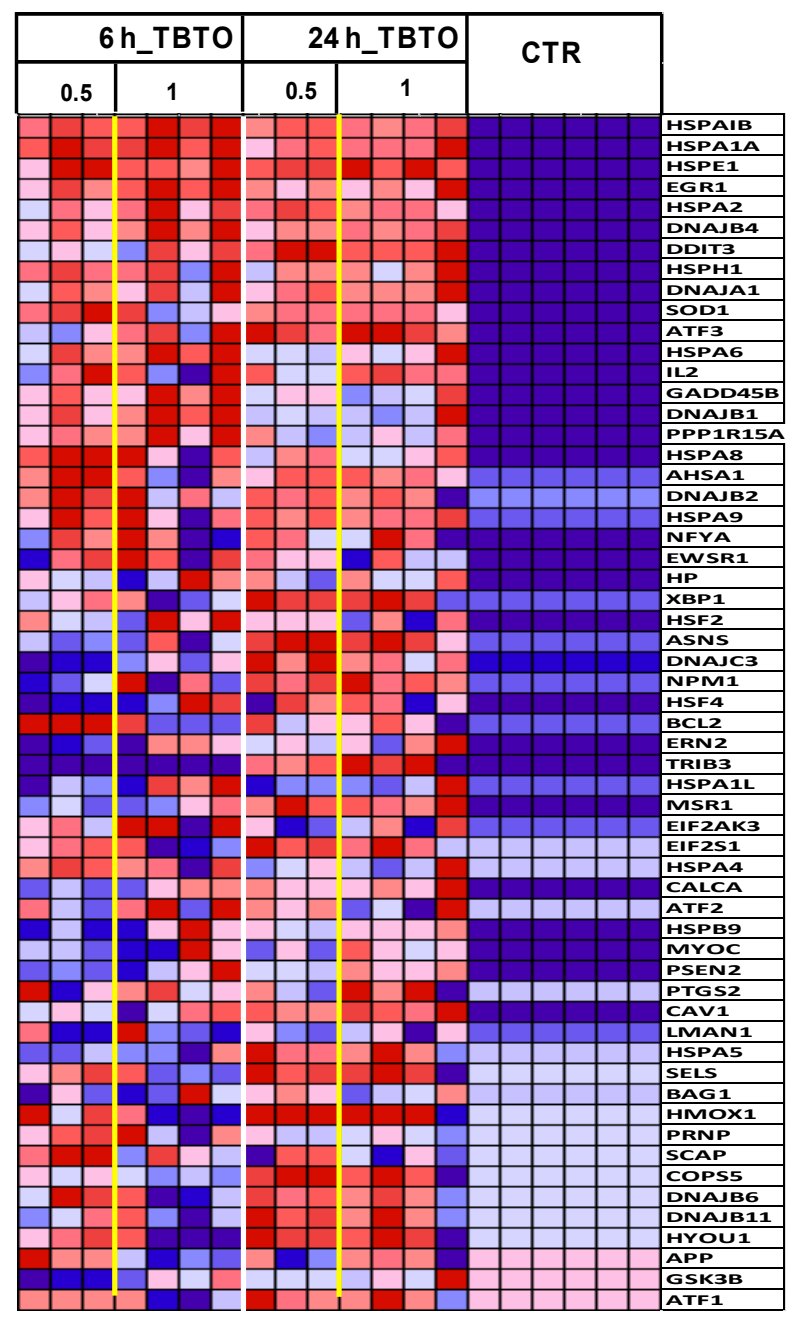


c.

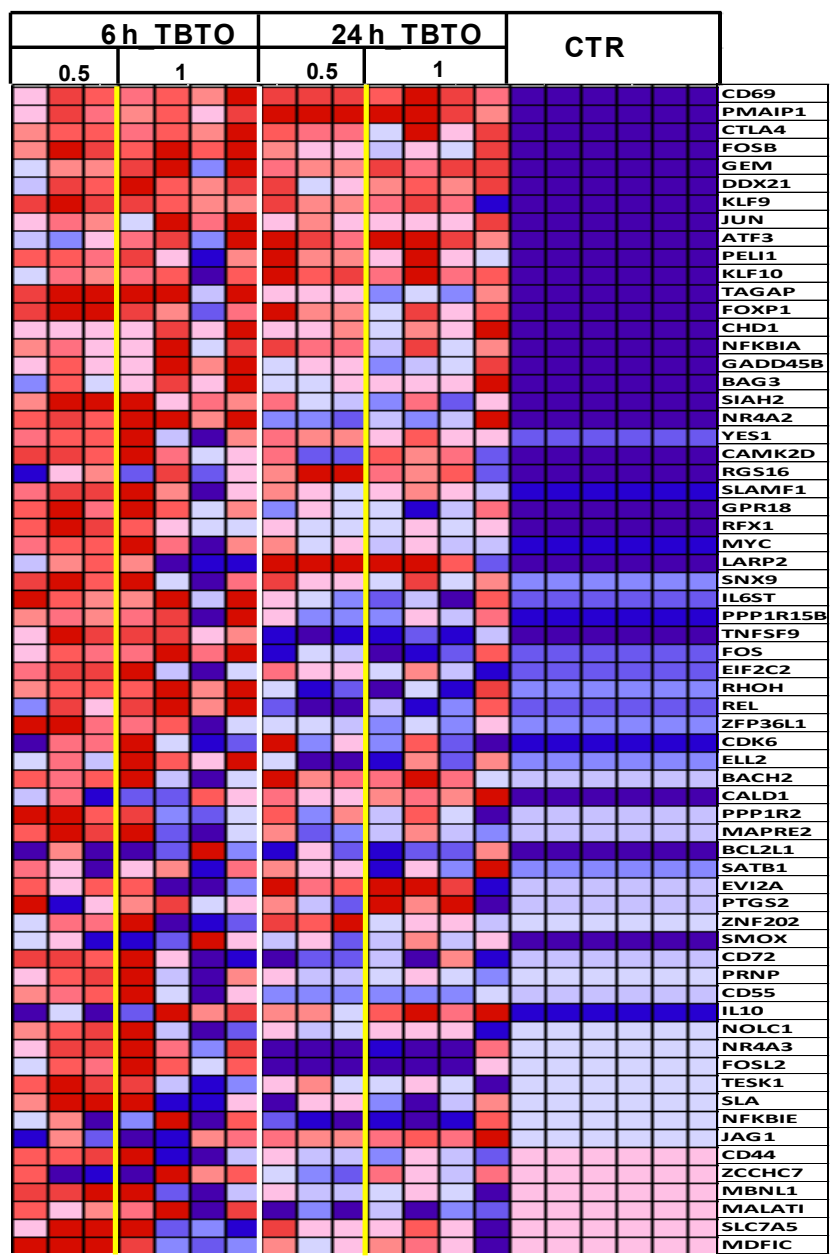


D.

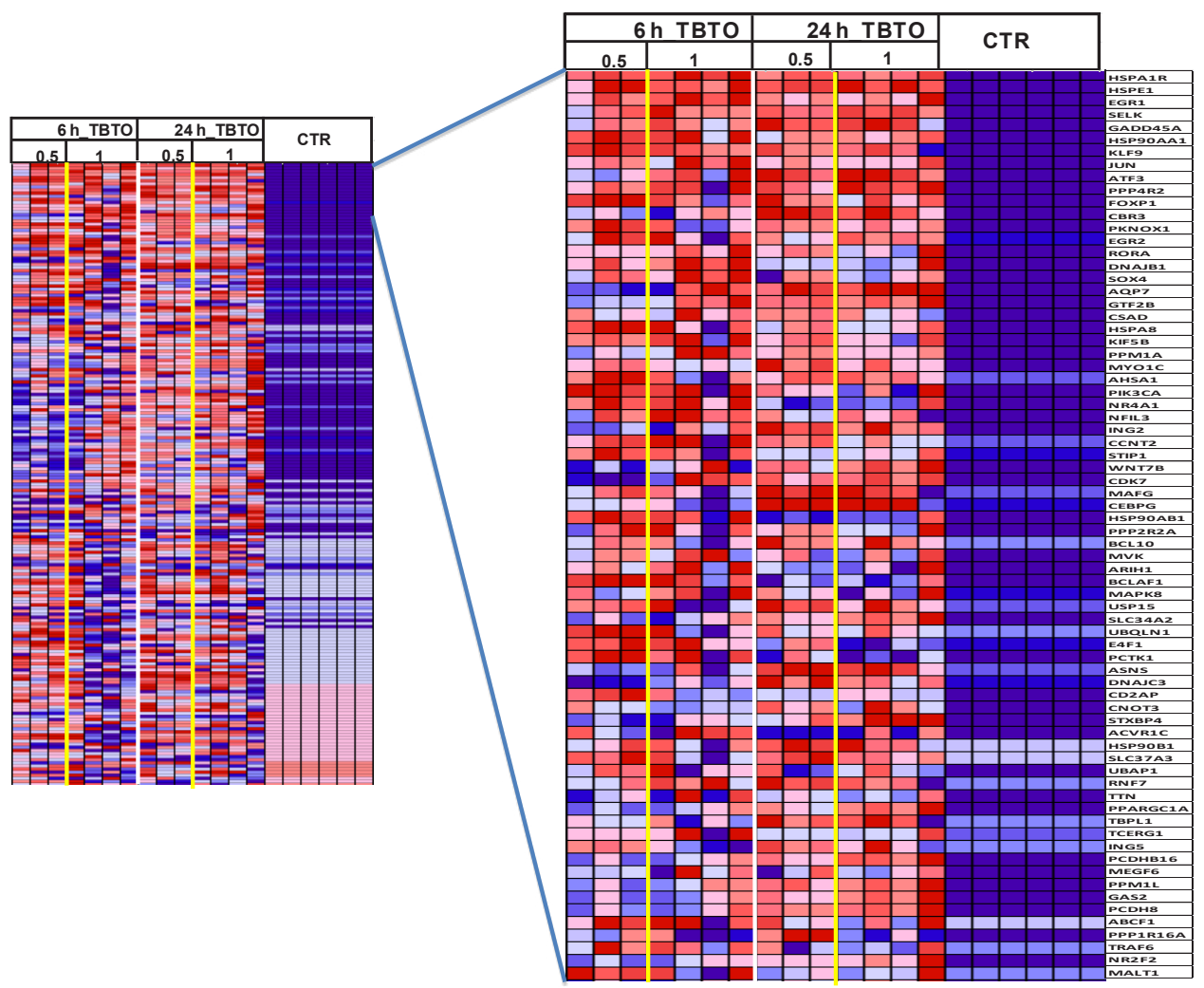


E.

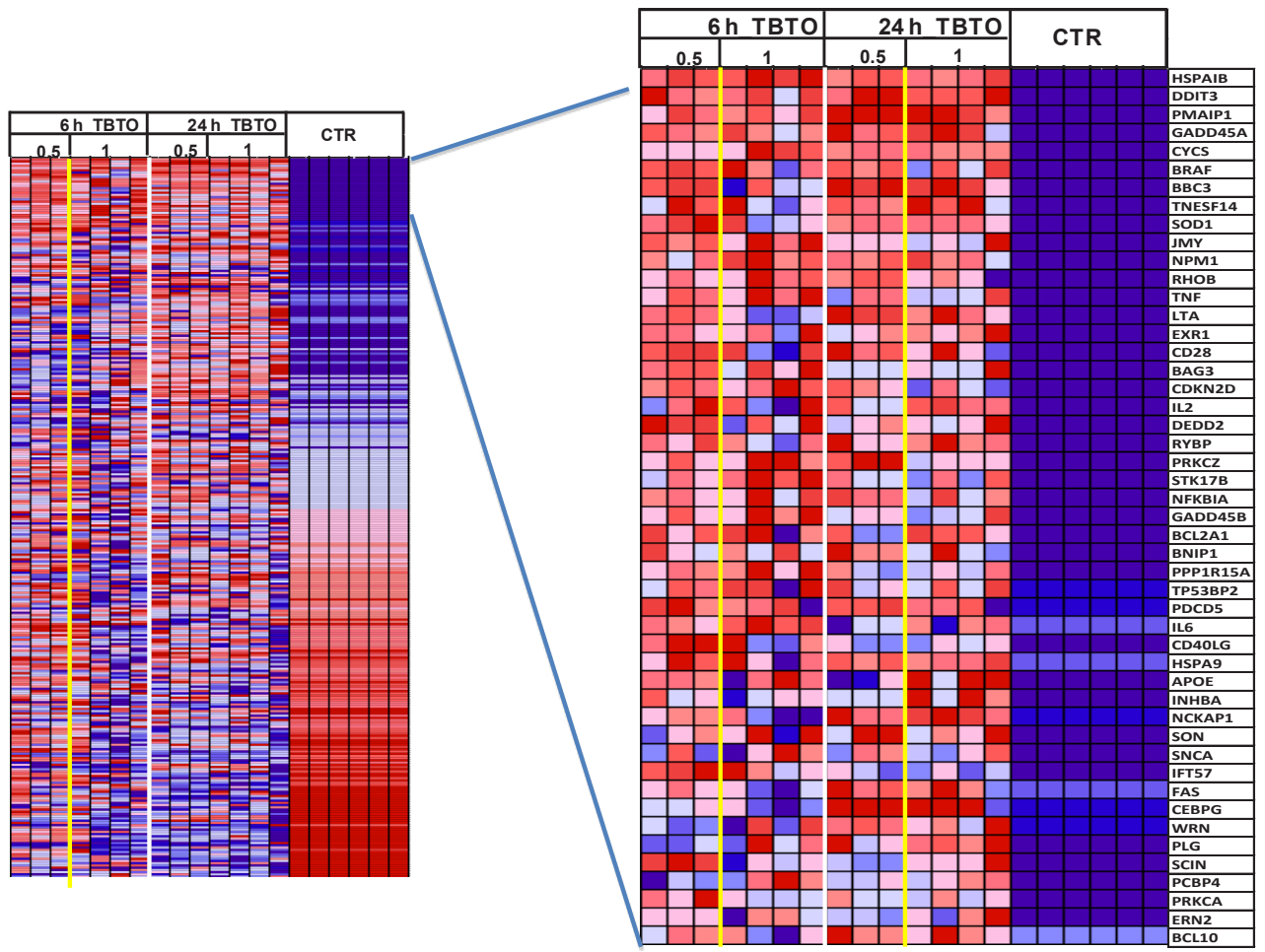

FIG. 4. TBTO modulates the expression of genes involved in translation, ER stress, $T$ cell activation, oxidative stress, and apoptosis. TBTO exposure results in differential expression of genes that are involved in A) Translation B) ER stress, C) T cell activation, D) oxidative stress, and E) apoptosis. The heat maps are based on $2 \log$ ratios of treatment versus the average of the control at the same time point. For visualization, six columns containing zeros were added, representing the relative expression of the control samples. Colors represent relative gene expression levels, blue indicates low expression, red indicates high expression.

\section{Comparative microarray data analysis}

We evaluated whether the pathways and biological processes affected by TBTO are not unique to PBMCs but are also affected in Jurkat cells. For this, we compared microarray data of PBMCs to those obtained before in microarray studies that examined the effect of TBTO on the transcriptome of Jurkat cells (Katika et al., 2012; Shao et al., 2013; Shao et al., submitted). A summary of the comparison of 
the data at pathway level is listed in Table 1. The comparison of the data of PBMCs and Jurkat cells at gene level is shown between in Supplementary fig. 3.

Next to PBMCs, ER stress related genes were clearly affected by TBTO in Jurkat cells with the higher doses inducing the most pronounced effects (Supplementary fig. 3a). A proportion of the ER stress related genes upregulated in PBMCs were downregulated in Jurkat cells.

NF-kB target genes were affected by TBTO in PBMCs and Jurkat cell (Supplementary fig. 3b).

Genes involved in oxidative stress and anti-oxidative response NRF2 pathway were affected in a comparable way in PBMCs and Jurkat cells (Supplementary fig. 3c). A part of genes upregulated in PBMCs is not upregulated in Jurkat cells.

T cell activation related genes are affected in PBMCs and Jurkat cells. (Supplementary fig. 3d). Many $\mathrm{T}$ cell activation related genes are upregulated in PBMCs but not in Jurkat cells or even downregulated in Jurkat cells.

Next to the PBMCs, TBTO upregulated pro-apoptotic genes and downregulated anti-apoptotic genes in PBMCs and Jurkat cells (Supplementary fig. 3e). Relatively high number of genes was affected in PBMCs.

Although comparative analysis showed similarities in the response of PBMCs and Jurkat cells to TBTO, a number of processes/gene sets related immune response were uniquely affected in PBMCs (Fig.2 and Table 1). These processes were mainly related to functions of PBMCs other than those of $T$ cells, i.e. NK cells, B cells, granulocytes, monocytes, dendritic cells, and macrophages (Fig.2 and Table 1) . 
TABLE 1. Overview of gene sets, processes, and pathways modulated by TBTO in PBMCs and Jurkat cells. Several processes were affected in PBMCs but not in Jurkat cells.

\begin{tabular}{|c|c|c|}
\hline Name gene set/process/pathway & PBMCs & Jurkat \\
\hline Translation & Fig. $4 \mathrm{~A}$ & Katika et al., 2012 \\
\hline ER stress & Fig. 4B & katika et al., 2012; Shao et al., 2013 \\
\hline oxidative stress & Fig. $4 \mathrm{C}$ & Katika et al., 2012; Shao et al., 2013 \\
\hline $\mathrm{T}$ cell activation & Fig. 4D & Katika et al., 2012 \\
\hline Apoptosis & Fig. $4 \mathrm{E}$ & Katika et al., 2012 \\
\hline Cell cycle & Suppl. Fig. 1a & Katika et al., 2012 \\
\hline RNA biosynthesis & Suppl. Fig. 1b & Shao et al., submitted \\
\hline Unfolded protein response (UPR) & Suppl. Fig. 1c & Katika et al., 2012; Shao et al., 2013 \\
\hline NF-kB pathway & Suppl. Fig. 1d & Katika et al., 2012 \\
\hline Mitochondria & Suppl. Fig. 1e & Shao et al., submitted \\
\hline Cytokine/chemokine signaling & Fig. 2 & Katika et al., 2012 \\
\hline CD40 signaling & Fig. 2 & Katika et al., 2012 \\
\hline Leukocyte chemotaxis & Fig. 2 & Shao et al., submitted \\
\hline TLR signaling & Fig. 2 & $x$ \\
\hline CD16 signaling in NK cells & Fig. 2 & $x$ \\
\hline B cell activation (PIP3 signaling) & Fig. 2 & $x$ \\
\hline Antigen presentation & Fig. 2 & $\mathrm{X}$ \\
\hline Transcriptional regulation in granulocytes & Fig. 2 & $x$ \\
\hline Granzyme A\&B signaling & Fig. 2 & $x$ \\
\hline t cell differentiation & Fig. 2 & $x$ \\
\hline Monocyte function & Fig. 2 & $x$ \\
\hline Dendritic cell maturation and function & Fig. 2 & $x$ \\
\hline PEG2 signaling & Fig. 2 & $x$ \\
\hline ICOS pathway in T helper cells & Fig. 2 & $x$ \\
\hline TREM1 signaling & Fig. 2 & $x$ \\
\hline Regulation of T cell function by CTLA4 & Fig. 2 & $x$ \\
\hline IL8 signaling neutrophils & Fig. 2 & $x$ \\
\hline
\end{tabular}

\section{Discussion}

Previously we have studied the effects of TBTO on the transcriptome of the human T lymphocyte cell line Jurkat (Katika et al., 2011; Shao et al., 2013). Biological interpretation of the microarray data revealed that various biological processes were affected upon treatment of this cell line with TBTO. The main purpose of the present study was to investigate whether TBTO would have similar effects on PBMCs. Since PBMCs contain lymphocyte subsets (T, B and NK cells), monocytes and dendritic cells, it allows to investigate the effects of TBTO on various immune cell types at the same time. To that end, PBMCs were exposed to TBTO $(0.5$ and $1 \mu \mathrm{M})$ for 6 and $24 \mathrm{~h}$ and subjected to whole genome expression profiling. Microarray data analysis showed that TBTO inhibits protein synthesis, induces 
ER stress, NFAT and NF-kB pathway as well as T cell activation, oxidative stress and apoptosis. Most of these processes were also found to be affected in Jurkat cells treated with TBTO (Katika et al., 2011; Shao et al., 2013).

Both Metacore and GSEA results revealed that TBTO upregulates genes related to RNA biosynthesis (Fig 2 and suppl.Fig. 1a). TBTO down regulates genes involved in translation (particularly after $24 \mathrm{~h}$ ) (Fig. 4A). These results indicate that TBTO inhibits protein synthesis, which correlate well with our findings on protein phosphorylation profiling of Jurkat cells that TBTO affected protein biosynthesis by inhibiting the p70S6K pathway starting from $3 \mathrm{~h}$ of exposure (Shao et al., submitted). The inhibition of protein synthesis by TBTO has also been reported by others in HEL-30 cells and EL4 cells (Marinovich et al., 1990; Osman et al., 2012).

TBTO clearly upregulated endoplasmic reticulum (ER) stress response genes according to Metacore and GSEA analysis (Fig. 4B). Previous genomic and immunocytological studies confirmed that TBTO primarily induces ER stress response in Jurkat cells (Katika et al., 2011; Shao et al., 2013; and Katika et al., 2013). Major ER stress genes including ATF3, DDIT3 and XBP1 are upregulated by TBTO in PBMCs and Jurkat cells. ATF3 is one of the early ER stress marker that is involved in the initiation of ER stress response and apoptosis (Holtz et al., 2006 and Li et al., 2006). DDIT3 (alias GADD153) is a C/EBP homologous transcription factor induces apoptotic genes during ER stress mediated apoptosis (Oyadomari et al., 2004). Pathways that are known to play a role in ER stress such as unfolded protein response and protein folding were also affected by TBTO (Suppl. Fig. 1a). The ER is known to be involved in the regulation of calcium homeostasis, protein folding and processing (Xu et al., 2005). ER stress is induced by accumulation of unfolded proteins and disturbance of $\mathrm{Ca}^{2+}$ homeostasis and cellular redox balance (Xu et al., 2005). The role of ER stress in the toxicity of TBTO was supported with ER stress inhibitor phenyl butyrate (PBA) which offered partial protection to TBTO induced impairment of cell viability in Jurkat cells (Katika et al., 2011).

Pathway analysis of the microarray data indicated that TBTO induces calcium mediated signaling (Fig. 2, cluster 4). Major genes involved in calcium mediated signalling (e.g. CALMI, CAMKIV, CAMK2D, FKBP14 and HDAC7) are upregulated by TBTO in PBMCs. Induction of intracellular free $\mathrm{Ca}^{2+}$ is a well-known effect of ER stress (Xu et al., 2005). Previously we confirmed the effect of TBTO on induction of intracellular calcium levels in Jurkat cells (Katika et al., 2012). The role of calcium in TBTO toxicity was supported by co-exposure experiments with the calcium chelator BAPTA in Jurkat cells showing protection against TBTO toxicity (Katika et al., 2011). Tributyltin (TBT) has also been reported to induce a substantial and rapid rise in intracellular $\mathrm{Ca}^{2+}$ in rat thymocytes, $\mathrm{B}$ lymphocytes and natural killer cells (Chow et al., 1992; Lane et al., 2009; Bissonnette et al., 2010).

The PBMC microarray data indicated that TBTO activates the NF-kB/ TNF- $\alpha$ pathway (Fig. 2, cluster D; Supplementary fig. 1c). The NF-kB pathway is known to be activated by ER stress, increased $\mathrm{Ca}^{2+}$ levels, and oxidative stress (Pahl and Baeuerle, 1996). Activation of NF-kB signaling by TBTO is also induced in Jurkat cells (Katika et al., 2011; Katika et al., 2012) and has also be reported for rat thymocytes (Nakano et al., 2004). 
TBTO also upregulates genes that are involved T cell activation, particularly after $6 \mathrm{~h}$ and much less after $24 \mathrm{~h}$ exposure (Fig. 4C). Pathways that are known to play a role in $\mathrm{T}$ cell activation, such as NFkB/ TNF- $\alpha /$ RELA/RELB and NFAT are upregulated by TBTO as well (Fig.2 and Supplementary fig.1c). The effect of TBTO on T cell activation was confirmed by qRT-PCR for two major early $T$ cell activation markers (CD69 and CD44) in Jurkat cells (Katika et al., 2013). Our Previous studies demonstrated that TBTO induces T cell activation at $3 \mathrm{~h}$ and downregulates after $6 \mathrm{~h}$ onwards which likely explain that Jurkat cells might undergo apoptosis quickly after induction of $T$ cell activation (Katika et al., 2011). Nuclear translocation of NFATC1 and activation of NF-kB(p65) was demonstrated by immunocytology (Katika et al., 2011; Katika et al., 2012). Most of the TBTO induced effects in PBMCs described above also occur during the normal $T$ cell activation, i.e., calcium release from the ER, calcium influx through CRAC channels, activation of calcineurin and NF-kB and translocation of NFAT to the nucleus (Gwack et al., 2007).

The microarray data indicated that TBTO upregulates genes involved in mitochondrial structure and function, in particular after $24 \mathrm{~h}$ exposure and a subset of genes are also downregulated (Supplementary fig. 1d). Previously, gene expression studies revealed that TBTO upregulates genes related to mitochondrial function and energy metabolism in time dependent manner in mouse thymocytes in vivo (Baken et al., 2007). In rat primary thymocytes, mitochondria related genes were upregulated at lower doses $(0.3$ and $0.5 \mu \mathrm{M})$ and downregulates at high doses $(1 \mu \mathrm{M})$ of TBTO. GSEA analysis of the transcriptome data of Jurkat cells indicated that TBTO downregulated the gene sets related to the function of mitochondrial inner membrane, ribosome, and electron transport at low concentration $(0.1 \mu \mathrm{M})$ after $6 \mathrm{~h}$ of exposure (Shao et al., submitted). It has been described before that TBT has an effect on mitochondria eventually leading to increased production of reactive oxygen species that in turn triggers NF-KB activation (Corsini et al., 1997). In the present experiment, TBTO treatment of PBMCs resulted clearly in upregulation of genes involved in the oxidative stress and the NRF2 antioxidant response pathway (Fig. 4D). The effect of TBTO on oxidative stress response has been demonstrated by immunocytological studies with Jurkat cells showing the activation of NRF2 and KEAP1 and the translocation of these proteins from the cytoplasm into the nucleus (Katika et al., 2011).

In PBMCs, TBTO clearly upregulated genes that are known to be involved in apoptosis (Fig. 4E). TBTO has been shown to upregulate apoptosis related genes and activate apoptosis related events such as M-calpain and caspase-3 in Jurkat cells (Katika et al.,2012). The induction of apoptosis by TBTO has been reported in rat thymocytes as well (Raffray et al., 1991; Raffray et al., 1993).

GSEA on the microarray data also indicated that TBTO downregulated genes that are highly expressed in lymphocyte subsets ( $T, B$ and NK cells), monocytes, dendritic cells and granulocytes in a dose and time dependent manner (Fig. 3) The effects were particularly more pronounced at higher doses ( $1 \mu \mathrm{M}$ TBTO) and at the longer time period ( $24 \mathrm{~h})$. The changes in gene expression profiles of lymphocytes subtypes, monocytes, dendritic cells and granulocytes is likely due to the result of two 
effects: (1) the shift in the proportion of the blood cell types, and (2) the effects on various biological processes in the blood cells (Table. 1).

Comparative analysis of microarray data indicates that most of the processes such as ER stress, T cell activation, NF-kB, oxidative stress, RNA biosynthesis, mitochondria, leukocyte chemotaxis, and apoptosis affected by TBTO are not unique to PBMCs but are also affected in Jurkat cells (Table 1, Supplementary fig. 3a-e). These findings support the modes of action of TBTO demonstrated by our previous study in the Jurkat T-cell line also take place in PBMCs. Despite this similarity in response, TBTO induced various immune response-related pathways in PBMCs such as dendritic cell maturation and function, Granzyme A\&B signaling, T cell differentiation, CD16 signaling in NK cells, TLR signaling, TREM1 signaling, PEG2 signaling, IL8 signaling in neutrophils, and monocyte function (Fig.2 and Table 1). The effects of TBTO on PBMCs are likely to be a mixture of responses of different immune cell types having their own gene expression.

In summary, the present study focused on identification of adverse outcome pathways and modes action of TBTO in human PBMCs and comparison of the data to those obtained with the human Jurkat $T$ cell line. Biological interpretation of microarray data revealed that TBTO inhibits protein synthesis, induces ER stress, NFAT, NF-kB/ TNF- $\alpha$, T cell activation response, oxidative stress and apoptosis. This study showed similar mode of action of TBTO detected before in the Jurkat cell line thereby confirming the mechanism of action of TBTO. The main mode of action of TBTO (ER stress) can be very well detected using Jurkat cells; but the application of PBMCs allows detection of additional toxic effects of TBTO on other immune cells then T cells, which cannot be detected using Jurkat cells (Table 1).

\section{Acknowledgements}

The study is supported by a grant (MFA 6809) that the University of Maastricht received from the Dutch Technology Foundation STW. 
A.

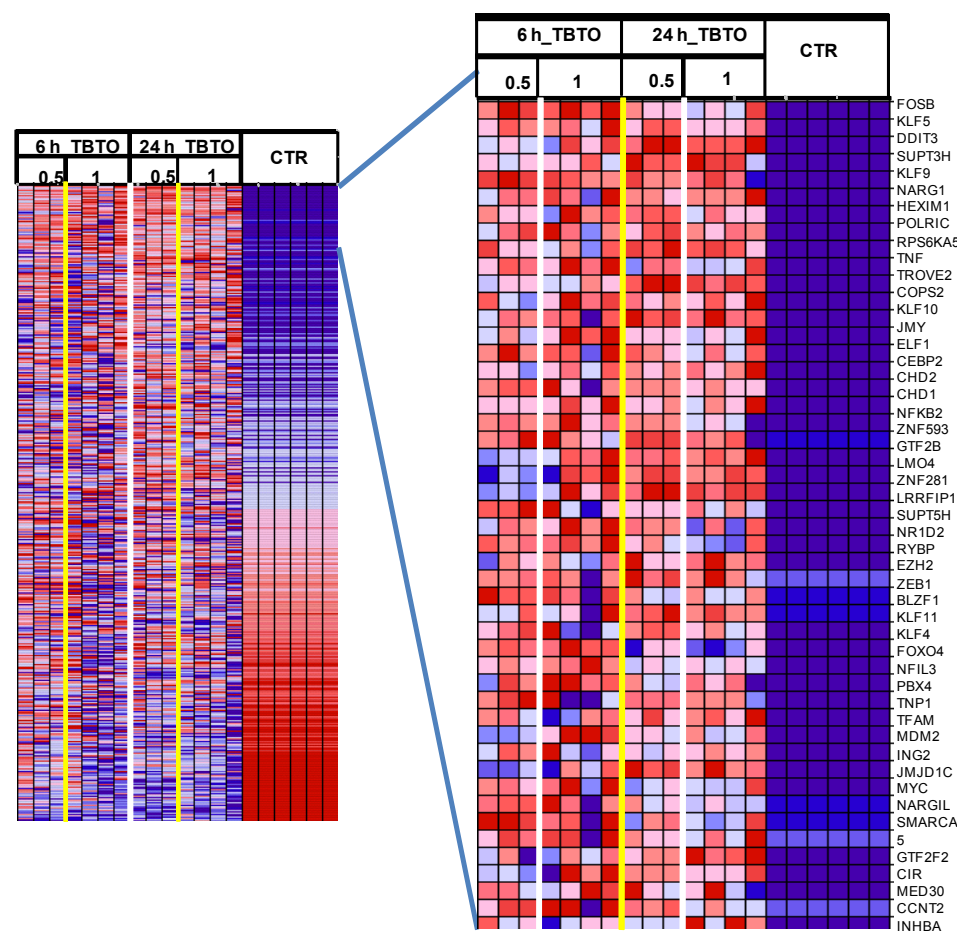

B.

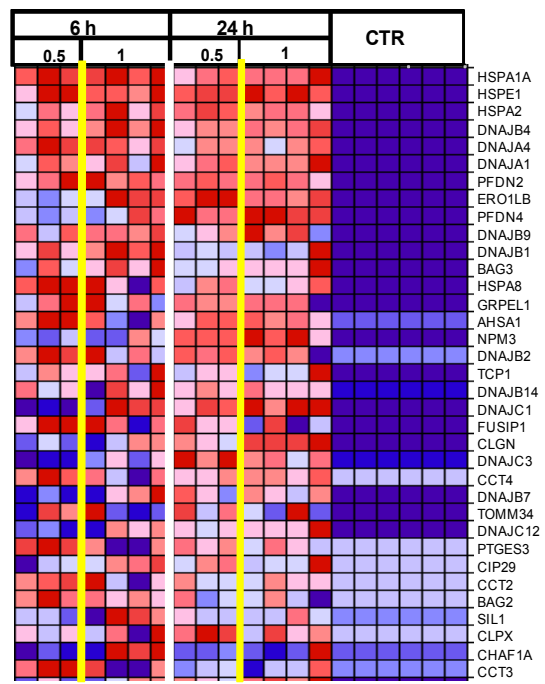

c.

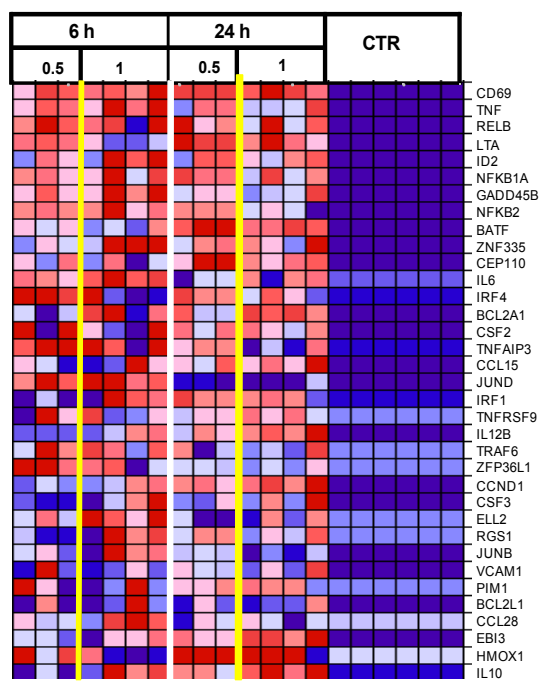


D.

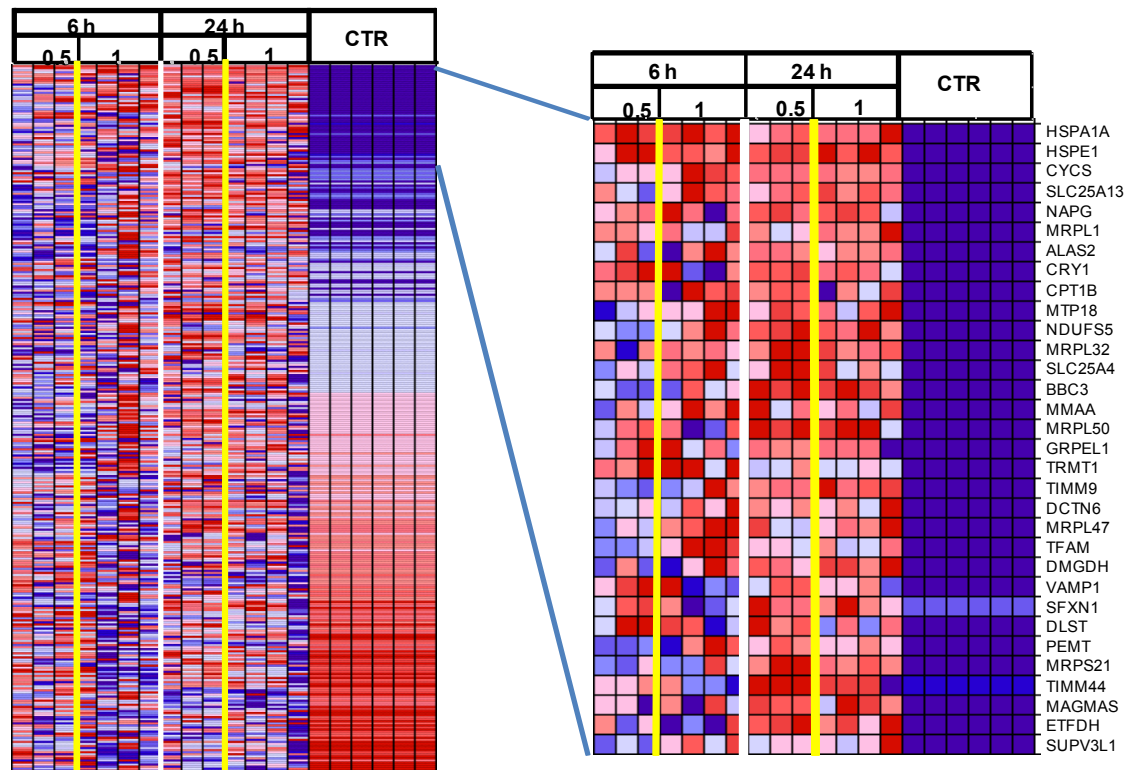

Supplementary fig. 1. TBTO modulates the expression of genes involved in RNA biosynthesis, unfolded protein response, NF-KB pathway, and mitochondria functions. TBTO exposure results in differential expression of genes that are involved in A) RNA biosynthesis, B) unfolded protein response, C) NF-кB pathway, D) mitochondria functions. The heat maps are based on 2 log ratios of treatment versus the average of the control at the same time point. For visualization, six columns containing zeros were added, representing the relative expression of the control samples. Colors represent relative gene expression levels, blue indicates low expression, red indicates high expression. 
A.

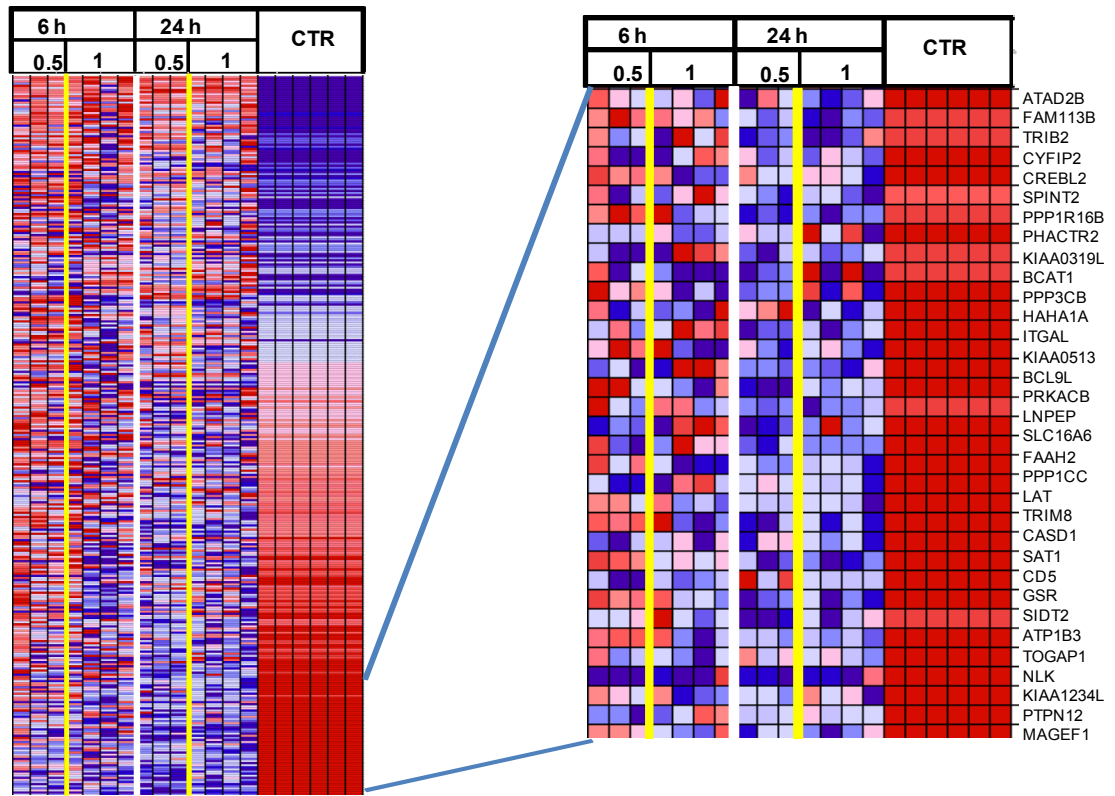

B.

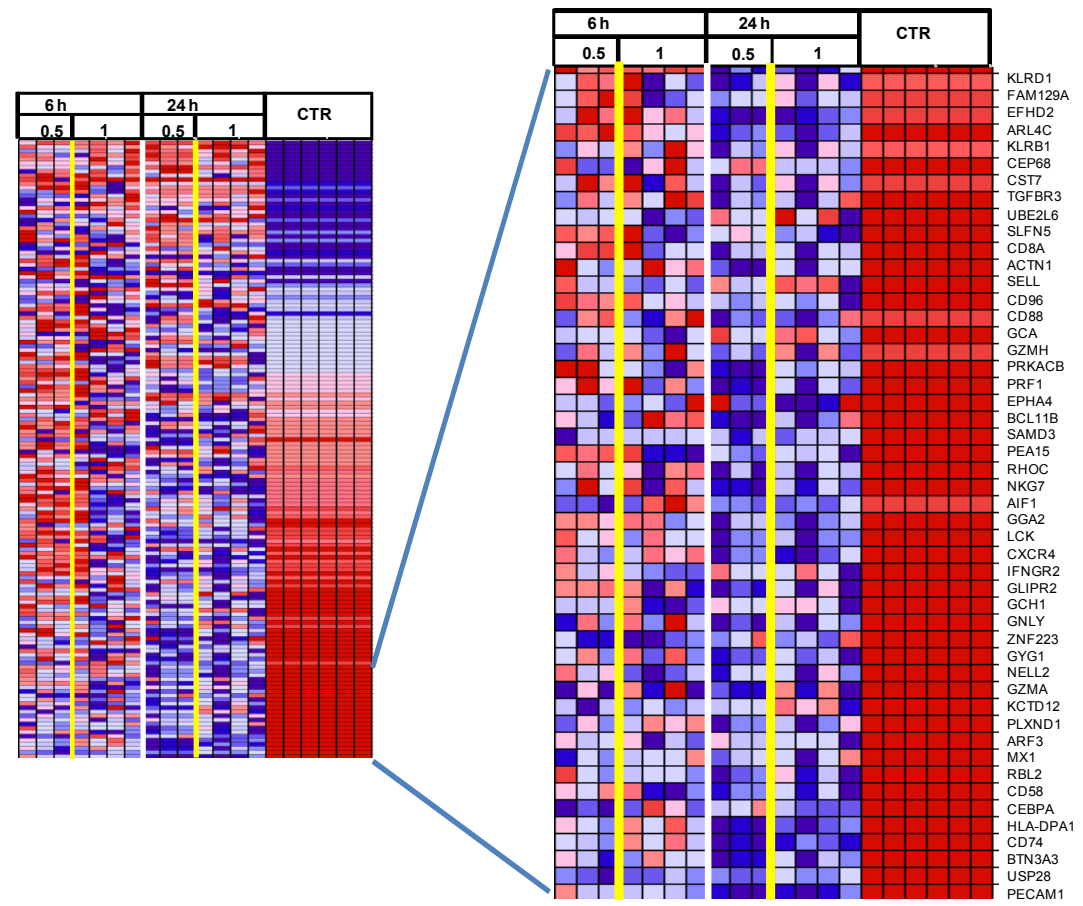


c.

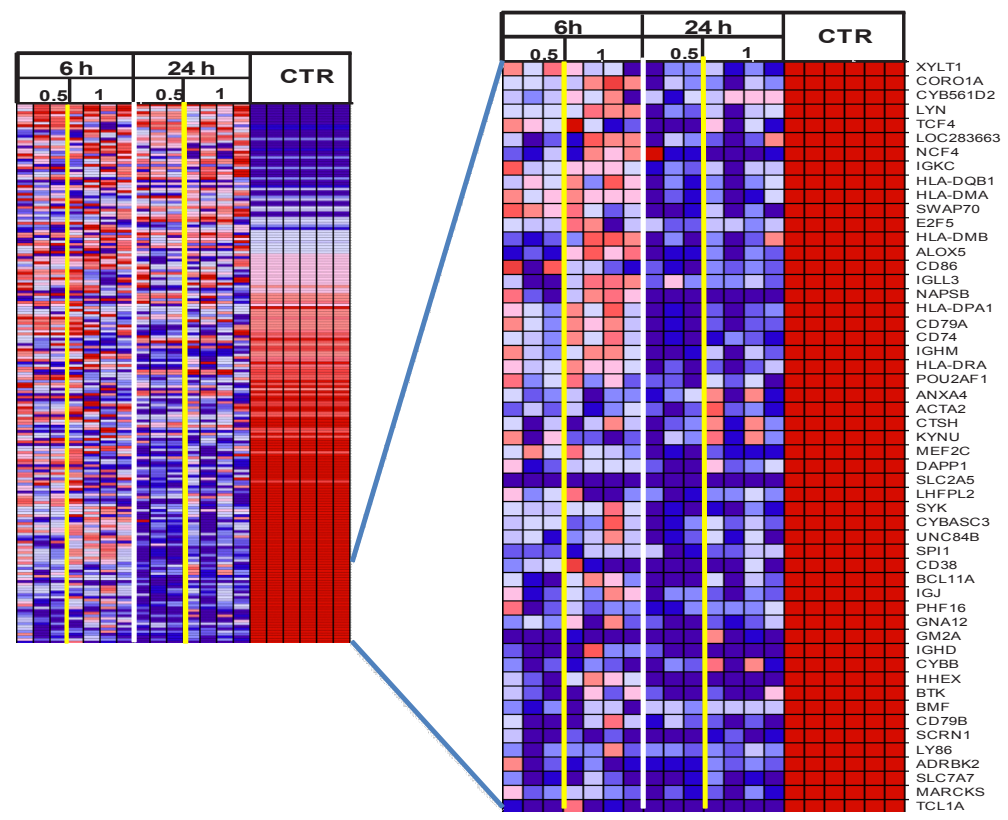

D.

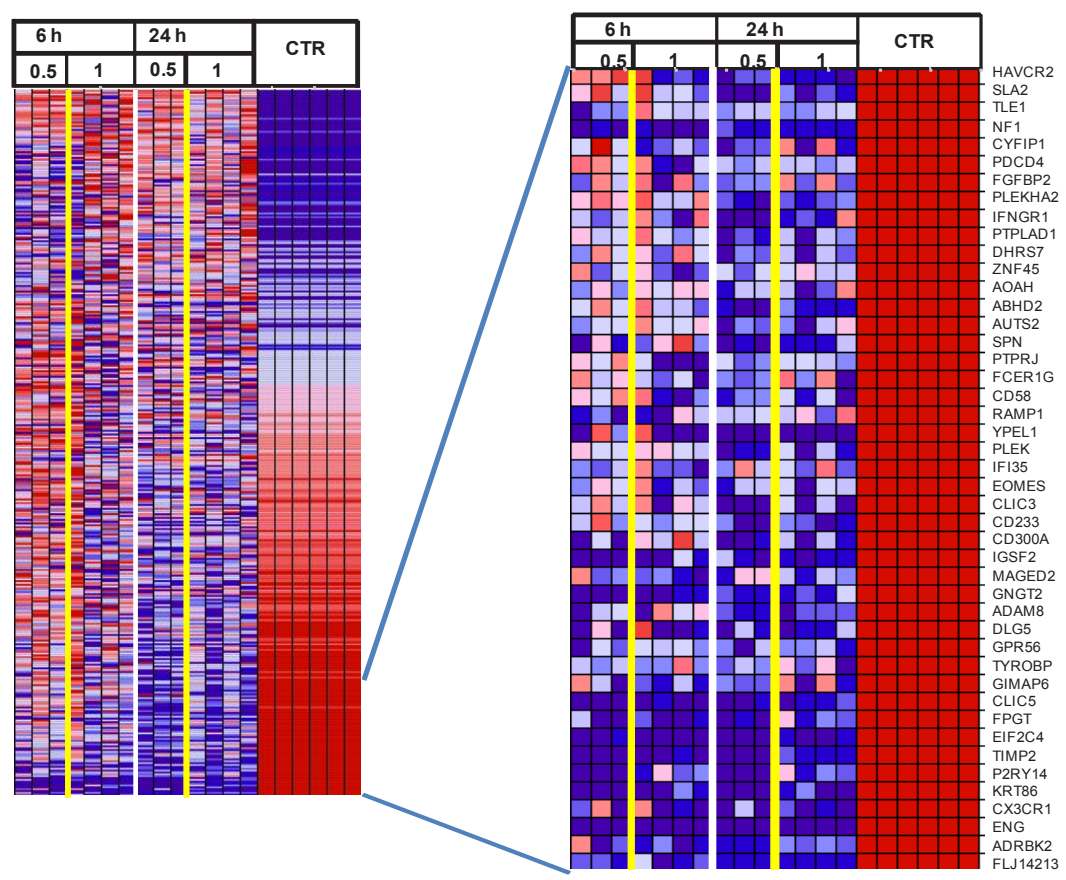


E.

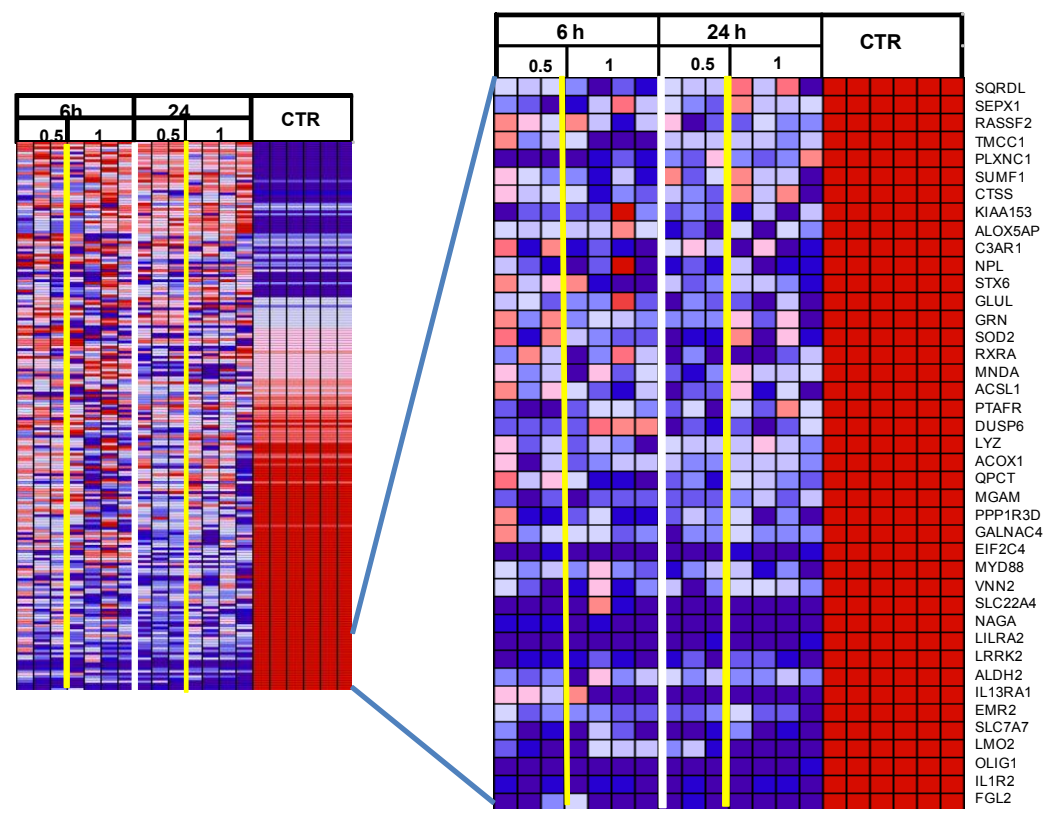

F.

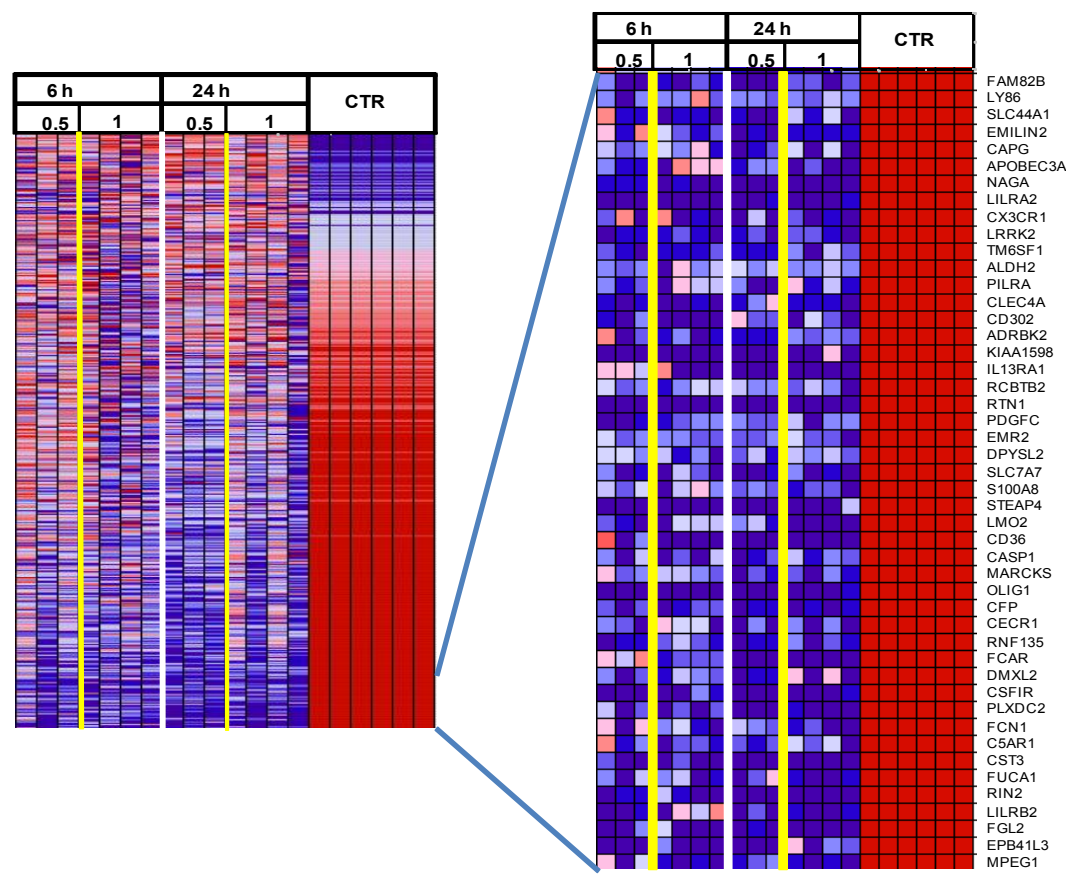


G.

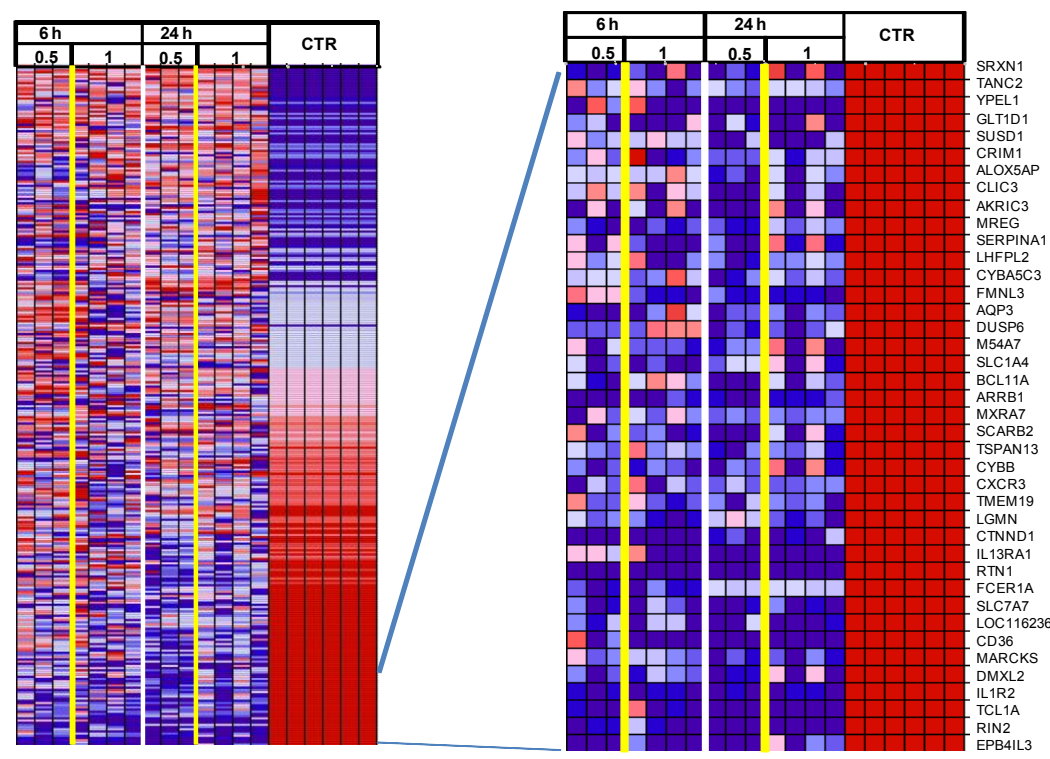

Supplementary fig. 2. TBTO downregulated the majority of genes that are related to $T$ cells $(C D 4+[A]$ and $C D 8+[B]), B$ cells $(C), N K$ cells $(D)$, granulocytes $(E)$, monocytes $(F)$ and dendritic cells $(G)$, particularly upon $24 \mathrm{~h}$ exposure. 
$\ddot{1}$

A.

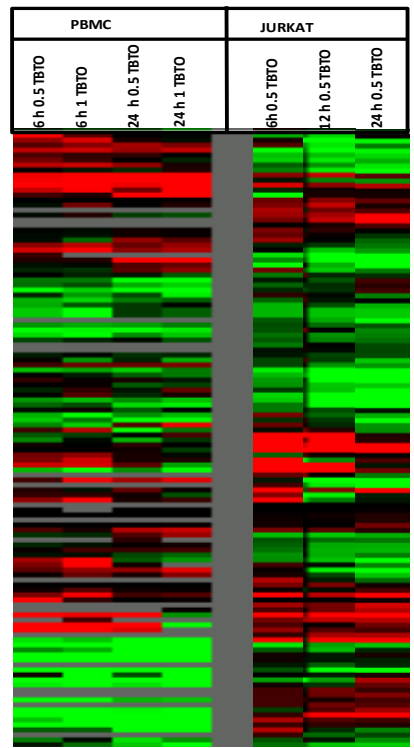

D.

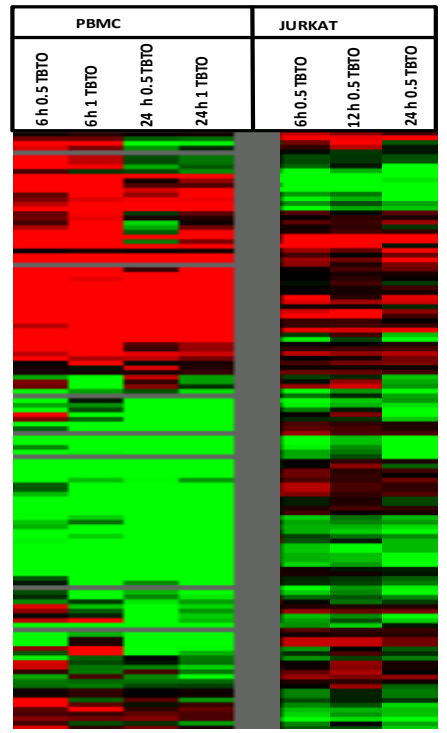

B.

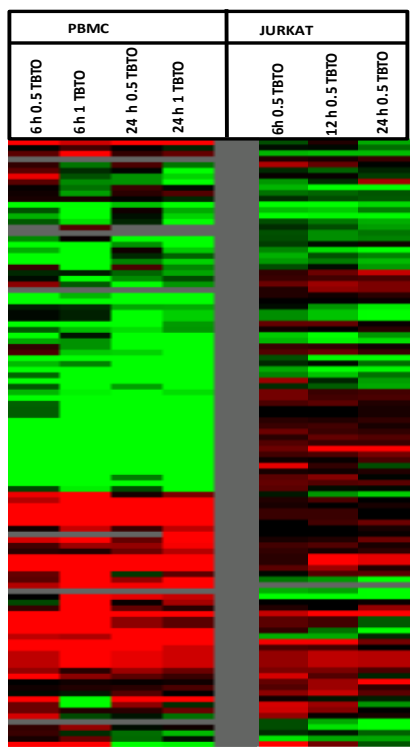

E.

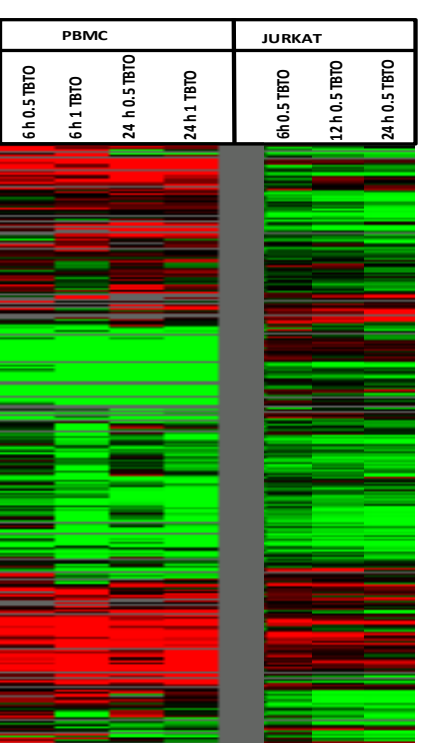

C.

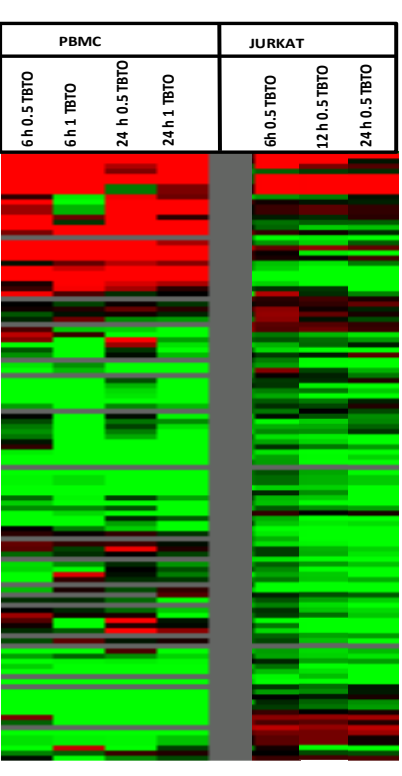

. 
Supplementary fig. 3. Comparison of the response of gene expression to TBTO exposure of PBMCs and Jurkat cells. Genes were selected on involvement in relevant pathways or processes: (A) ER stress, (B) NF-kB, (C) oxidative stress; (D) $T$ cell activation, and (E) apoptosis. Heatmaps were made on 2log ratios of treatment vs. average of the controls. Green indicates down regulation and red upregulation. Black indicates no significant effect. Genes were filtered on up- or downregulation >1.7-fold in at least 1 of 12 samples. 


\section{References}

Aluoch, A.O., Odman-Ghazi, S.O. and Whalen, M.M. 2007. Pattern of MAP kinases p44/42 and JNK activation by non-lethal doses of tributyltin in human natural killer cells. Arch Toxicol 81, 271-277.

Antizar-Ladislao, B. 2008. Environmental levels, toxicity and human exposure to tributyltin (TBT)contaminated marine environment. a review. Environ Int 34, 292-308.

Baken, K.A., Arkusz, J., Pennings, J.L., Vandebriel, R.J. and van Loveren, H. 2007. In vitro immunotoxicity of bis(tri-n-butyltin)oxide (TBTO) studied by toxicogenomics. Toxicology 237, 35-48.

Berg, C.P., Rothbart, A., Lauber, K., Stein, G.M., Engels, I.H., Belka, C., Janicke, R.U., SchulzeOsthoff, K. and Wesselborg, S. 2003. Tributyltin (TBT) induces ultra-rapid caspase activation independent of apoptosome formation in human platelets. Oncogene 22, 775-780.

Bissonnette, S.L., Haas, A., Mann, K.K. and Schlezinger, J.J. 2010. The role of CaMKII in calciumactivated death pathways in bone marrow B cells. Toxicological sciences 118, 108-118.

Camps, M., Briand, J.F., Guentas-Dombrowsky, L., Culioli, G., Bazire, A. and Blache, Y. 2011. Antifouling activity of commercial biocides vs. natural and natural-derived products assessed by marine bacteria adhesion bioassay. Mar Pollut Bull.

Chow, S.C., Kass, G.E., McCabe, M.J., Jr. and Orrenius, S. 1992. Tributyltin increases cytosolic free $\mathrm{Ca} 2+$ concentration in thymocytes by mobilizing intracellular $\mathrm{Ca} 2+$, activating a $\mathrm{Ca} 2+$ entry pathway, and inhibiting Ca2+ efflux. Arch Biochem Biophys 298, 143-149.

Eisen, M.B., Spellman, P.T., Brown, P.O. and Botstein, D. 1998. Cluster analysis and display of genome-wide expression patterns. P Natl Acad Sci USA 95, 14863-14868.

Ekins, S., Bugrim, A., Brovold, L., Kirillov, E., Nikolsky, Y., Rakhmatulin, E., Sorokina, S., Ryabov, A., Serebryiskaya, T., Melnikov, A., Metz, J. and Nikolskaya, T. 2006. Algorithms for network analysis in systems-ADME/Tox using the MetaCore and MetaDrug platforms. Xenobiotica 36, 877-901.

Fent, K. 1996. Ecotoxicology of organotin compounds. Crit Rev Toxicol 26, 1-117.

Granmo, A., Ekelund, R., Sneli, J.A., Berggren, M. and Svavarsson, J. 2002. Effects of antifouling paint components (TBTO, copper and triazine) on the early development of embryos in cod (Gadus morhua L.). Mar Pollut Bull 44, 1142-1148.

Grundler, W., Dirscherl, P., Beisker, W., Marx, K., Stampfl, A., Maier, K., Zimmermann, I. and Nusse, M. 2001. Early functional apoptotic responses of thymocytes induced by Tri-n-butyltin. Cytometry 44, 45-56.

Gwack, Y., Feske, S., Srikanth, S., Hogan, P.G. and Rao, A. 2007. Signalling to transcription: storeoperated Ca2+ entry and NFAT activation in lymphocytes. Cell Calcium 42, 145-156.

Inadera, H. 2006. The immune system as a target for environmental chemicals: Xenoestrogens and other compounds. Toxicology Letters 164, 191-206.

Katika, M.R., Hendriksen, P.J., van Loveren, H. and Peijnenburg, A. 2011. Exposure of Jurkat cells to bis (tri-n-butyltin) oxide (TBTO) induces transcriptomics changes indicative for ER- and oxidative stress, T cell activation and apoptosis. Toxicol Appl Pharm 254, 311-322. 
Katika, M.R., Hendriksen, P.J., de Ruijter N. C., van Loveren, H. and Peijnenburg, A. 2012. Immunocytological and biochemical analysis of the mode of action of bis(tri-n-butyltin)tri-oxide (TBTO) in Jurkat cells. Toxicol Lett 212 (2), 126-136.

Lane, R., Ghazi, S. and Whalen, M. 2009. Increases in Cytosolic Calcium lon Levels in Human Natural Killer Cells in Response to Butyltin Exposure. Archives of Environmental Contamination and Toxicology 57, 816-825.

Nakano, K., Tsunoda, M. and Konno, N. 2004. Tributyltin (TBT) Increases TNF \&alpha; mRNA Expression and Induces Apoptosis in the Murine Macrophage Cell Line in Vitro. Environmental Health and Preventive Medicine 9, 266-271.

Osman, A.M., Pennings, J.L.A., Blokland, M., Peijnenburg, A. and van Loveren, H. 2010. Protein expression profiling of mouse thymoma cells upon exposure to the trichothecene deoxynivalenol (DON): Implications for its mechanism of action. Journal of Immunotoxicology 7, 147-156.

Pahl, H.L. and Baeuerle, P.A. 1996. Activation of NF-kappa B by ER stress requires both $\mathrm{Ca}+$ and reactive oxygen intermediates as messengers. FEBS Letters 392, 129-136.

Raffray, M. and Cohen, G.M. 1991. Bis(tri-n-butyltin)oxide induces programmed cell death (apoptosis) in immature rat thymocytes. Arch Toxicol 65, 135-139.

Raffray, M., Mccarthy, D., Snowden, R.T. and Cohen, G.M. 1993. Apoptosis as a Mechanism of Tributyltin Cytotoxicity to Thymocytes - Relationship of Apoptotic Markers to Biochemical and Cellular Effects. Toxicol Appl Pharm 119, 122-130.

Reich, M., Liefeld, T., Gould, J., Lerner, J., Tamayo, P. and Mesirov, J.P. 2006. GenePattern 2.0. Nat Genet 38, 500-501.

Shaffer, A.L., Rosenwald, A., Hurt, E.M., Giltnane, J.M., Lam, L.T., Pickeral, O.K. and Staudt, L.M. 2001. Signatures of the Immune Response. Immunity 15, 375-385.

Snoeij, N.J., Penninks, A.H. and Seinen, W. 1987. Biological activity of organotin compounds--an overview. Environ Res 44, 335-353.

Snoeij, N.J., Penninks, A.H. and Seinen, W. 1988. Dibutyltin and tributyltin compounds induce thymus atrophy in rats due to a selective action on thymic lymphoblasts. Int J Immunopharmacol 10, 891-899. Sroka, J., Wlosiak, P., Wilk, A., Antonik, J., Czyz, J. and Madeja, Z. 2008. The effect of tributyltin on human eosinophilic [correction of eosinophylic] leukemia EoL-1 cells. Cell Mol Biol Lett 13, 67-73.

Takahashi, S., Mukai, H., Tanabe, S., Sakayama, K., Miyazaki, T. and Masuno, H. 1999. Butyltin residues in livers of humans and wild terrestrial mammals and in plastic products. Environmental Pollution 106, 213-218.

Whalen, M.M., Dudimah, F.D., Abraha, A. and Wang, X.F. 2010. Activation of p44/42 in human natural killer cells decreases cell-surface protein expression: Relationship to tributyltin-induced alterations of protein expression. Toxicol Mech Method 20, 544-555.

Xu, C.Y., Bailly-Maitre, B. and Reed, J.C. 2005. Endoplasmic reticulum stress: cell life and death decisions. J Clin Invest 115, 2656-2664. 


\section{SECTION II}

Identification and validation of functional biomarkers for predictive immunotoxicity tesing 



\section{CHAPTER 6}

\section{Transcriptome-based functional classifiers for direct immunotoxicity}

Jia Shao, Laura F. Berger, Peter J. M. Hendriksen, Ad A. C. M. Peijnenburg Henk van Loveren, and Oscar L. Volger

Arch. Toxicol. 2014, 88: 673-689

Supplementary materials can be found at: http://link.springer.com/article/10.1007\%2Fs00204-013-1179-1 


\begin{abstract}
Current screening methods for direct immunotoxic chemicals are mainly based on general toxicity studies with rodents. The present study aimed to identify transcriptome-based functional classifiers that can eventually be exploited for the development of in vitro screening assays for direct immunotoxicity. To this end, a toxicogenomics approach was applied in which gene expression changes in human Jurkat lymphoblastic $T$ cells were investigated in response to a wide range of compounds, including direct immunotoxicants, immunosuppressive drugs, and non-immunotoxic control chemicals. On the basis of DNA microarray data previously obtained by exposure of Jurkat cells to 31 test compounds (Shao et al., in Toxicol. Sci. 135(2): 328-346, 2013), we identified a set of 93 genes, of which 80 were significantly regulated (|numerical ratio| $\geq 1.62$ ) by at least three compounds and the other 13 genes were significantly regulated by either one single compound or compound class. A total of 28 most differentially regulated genes were selected for qRT-PCR verification using a training set of 44 compounds consisting of the above mentioned 31 compounds (23 immunotoxic and 8 non-immunotoxic) and 13 additional immunotoxicants. Good correlation between the results of microarray and qRT-PCR (Pearson's correlation, $R \geq 0.69$ ) was found for 27 out of the 28 genes. Redundancy analysis of these 27 potential classifiers led to a final set of 25 genes. To assess the performance of these genes, Jurkat cells were exposed to 20 additional compounds (external verification set) followed by qRT-PCR. The classifier set of 25 genes gave a good performance in the external verification: accuracy $85 \%$, true positive rate (sensitivity) $88 \%$, and true negative rate (specificity) $67 \%$. Furthermore, on the basis of the gene ontology (GO) annotation of the 25 classifier genes, the immunotoxicants examined in this study could be categorized into distinct functional subclasses. In conclusion, we have identified and validated classifier genes that can be used for the development of an in vitro assay for the identification and initial characterization of hazards for direct immunotoxicity of chemicals and drugs. This assay promises to complement animal free toxicity testing approaches within the field of direct immunotoxicity.
\end{abstract}




\section{Introduction}

Immunotoxicity is defined as the adverse effects of a xenobiotic on the functioning of the immune system. Two types of immunotoxicity can be distinguished, i.e., direct and indirect immunotoxicities (De Jong and van Loveren, 2007; Lankveld et al., 2010). Direct immunotoxicity refers to the toxicological effect of a compound on components of the immune system leading to immunosuppression or immunostimulation. Indirect immunotoxicity is triggered by specific immune response to a compound itself or to a self-antigen altered by the compound, resulting in hypersensitivity or autoimmunity (Lankveld et al., 2010). The assessment of direct immunotoxicity is an important component of the overall toxicological investigation of chemicals (Lankveld et al., 2010). Guidelines from the EMA (2000) and FDA/ CDER (2002) emphasized the need to evaluate immunotoxic effects during the preclinical phase of drug development, while similar guidelines have also been issued by OECD (Institóris et al., 1998) and EPA (1998) with respect to the safety assessment of chemicals and food additives. Current methods for screening direct immunotoxicity are mainly based on in vivo toxicity studies with rodent-based animal models. A tiered approach has been recommended for these in vivo tests with TIER 1 being a 28-day repeated dose toxicity study with emphasis on the evaluation of immune-related organs. TIER 2 consists of several immunological experiments, in order to (1) investigate the effects of chemicals on immune functionality, (2) determine the extent of the immunotoxicity, and (3) to identify the specific functional target in the immune system (De Jong and van Loveren, 2007). However, the current animal-based approach has several disadvantages, such as its limited prediction toward the human situation due to rodent-human interspecies differences (Kawabata and Evans, 2012), the time- and money-consuming nature of animal experimentation, and the ethical concerns being raised against animal experiments in society. Another essential problem is that the accuracy of animal tests for toxicity of chemicals or pharmaceuticals is rather low, which is estimated as 37-50\% (Perel et al., 2007). Regarding pharmaceuticals, there has been much concern on adverse drug reactions. This is due to the fact that many drugs that successfully passed the animal tests turned out to give serious adverse effects in humans. It has been estimated in 2008 that 197,000 EU citizens were killed annually because of that (Archibald et al., 2011). Therefore, the development of in vitro testing methods as alternatives for animal models of human toxicity is being promoted by EU policy (Bouvier d'Yvoire et al., 2012).

For many immunotoxic compounds, the precise mechanisms underlying toxicity are not understood. The application of genomics in immunotoxicology, also referred to as immunotoxicogenomics, may be able to fill this gap (Luebke et al., 2006). Immunotoxicogenomics can provide insights into the modes of action by analyzing chemical-induced changes in gene expression patterns. In addition, gene expression profiling has been demonstrated to be a useful tool for the characterization of structural and functional compound classes and the development of new biomarkers for toxicity endpoints (Tugwood et al., 2003; Waters and Fostel, 2004).

In a previous study, we carried out DNA microarray experiments with the human Jurkat $T$ cell line in order to identify mechanisms underlying direct immunotoxicity (Shao et al., 2013). The Jurkat cell line 
was chosen as in vitro model system since it has frequently been used successfully in traditional in vitro studies of the immune system, such as the assessment of mitogenic stimuli (Makar et al., 1994; Daaka et al., 1996; Fernández-Riejos et al., 2008) and cell migration (Fischer et al., 2004). Recent toxicogenomics studies in our laboratory have also demonstrated that the responses of Jurkat cells to immunotoxic compound deoxynivalenol are comparable with the responses of human PBMCs (Katika et al., 2012). Furthermore, Jurkat is a cell line, which makes experiments less labor-intensive and more reproducible as compared to primary cells. Moreover, Jurkat cells are of human origin, therefore facilitating extrapolation to the human situation. In our previous study, Jurkat cells were exposed to 31 test compounds including direct immunotoxicants, immunosuppressive drugs with various modes of action, and non-immunotoxic chemicals. Several general processes including response to ER stress, response to oxidative stress, regulation of cell cycle, and anti-apoptosis were found to be commonly affected by the immunomodulating compounds. Processes including responses to hypoxia and retinoic acid were found to be specifically affected by one or two of the immunomodulating compounds (Shao et al., 2013).

In the present work, these array data were used to select candidate classifier genes that are capable to distinguish between direct immunotoxic and non-immunotoxic compounds. For this aim, we started with a set of 27 most differentially regulated genes in our first study that are involved in a wide range of modes of action leading to immunotoxicity. The microarray results of these 27 genes have been confirmed by quantitative real-time PCR (qRT-PCR) (Shao et al., 2013). For the present study, we exposed Jurkat cells to 13 other immunotoxicants, which was followed by qRT-PCR on the set of 27 genes. These 13 newly tested immunotoxicants, together with the 31 compounds tested before (23 immunotoxic and 8 non-immunotoxic), were used as a training set. A set of another 20 compounds (17 immunotoxic and 3 non-immunotoxic) was used as an external verification set. The training set of 44 compounds was used to identify candidate classifier genes for direct immunotoxicity and to perform a redundancy analysis in order to determine the optimal number of classifier genes that is required to accurately distinguish between immunotoxic and non-immunotoxic compounds. The external verification set of 20 compounds was used to check the statistical power of the classifiers identified with the training set. The results demonstrated that detection of changes in mRNA levels of a set of 25 classifier genes is sufficient to yield a good prediction (85\% accuracy) of immunotoxic effects of compounds. This set of classifiers can potentially be used as an in vitro high throughput screen that employs mRNA expression profiling in order to detect possible direct immunotoxic properties of chemicals and drugs, and to categorize these direct immunotoxicants into distinct functional subclasses.

\section{Materials and Methods}

Cell culture. The human lymphoblastic T-cell line (Jurkat) was obtained from the American Type Culture Collection (ATCC). Cells were grown at $37^{\circ} \mathrm{C}$ in a humidified atmosphere with $5 \% \mathrm{CO}_{2}$, in RPMI-1640 medium supplemented with $10 \%$ heat inactivated fetal calf serum (FCS), $2 \mathrm{mM}$ glutamine, 156 
$1 \mathrm{mM}$ sodium pyruvate, $1 \mathrm{mM}$ nonessential amino acid, $100 \mathrm{U} / \mathrm{ml}$ penicillin, and $100 \mu \mathrm{g} / \mathrm{ml}$ streptomycin. The cultures were maintained in T-75 flasks (Gibco, Breda, The Netherlands), and the medium was refreshed every 2 days.

Compound selection. The criteria used for the selection of the direct immunotoxicants were the severity of immunotoxicity and the environmental abundance. Besides environmental contaminants, we also incorporated a group of immunosuppressive drugs with well-defined molecular targets, which include DNA alkylation, glucocorticoid receptor activation, inhibition of purine synthesis, calcineurin, mTOR, sphingosine-1-phosphate receptor 1 (S1PR1), and cyclooxygenase 2 (COX-2). See Table 1 for the complete chemical list of both training and external verification sets. Mono-2-ethylhexyl phthalate (MEHP) was purchased from Wako chemicals, Neuss, Germany. Fingolimod (FTY720) was purchased from Selleck Chemicals, via Bio-Connect Diagnostics, Huissen, The Netherlands. 2-Amino3-methyl-3H-imidazo[4,5-F]quinoline (IQ) and 2-amino-1-methyl-6-phenylimidazo[4,5-b]pyridine (PhIP) were purchased from Santa Cruz via Tebu-Bio, Heerhugowaard, The Netherlands. 4-Hydroxy-2nonenal (4-HNE) was purchased from Merck, Darmstadt, Germany. Okadaic acid (OA) was purchased from Calbiochem, Roden, The Netherlands. Other chemicals were purchased from Sigma-Aldrich (Zwijndrecht, The Netherlands). Stock solutions of all chemicals were made in dimethyl sulfoxide (DMSO; Merck, Darmstadt, Germany), except for DMNA, which was received as a ready-to-use stock in methanol.

Metabolic activation system. Aflatoxin B1, benzo[a]pyrene, cyclophosphamide, and ochratoxin A need to be biotransformed before exerting their immunomodulatory effects (Carlson et al., 2004; Ekhart et al., 2009; Manderville, 2005; Ramsdell et al., 1991). Therefore, these compounds were bioactivated in vitro using human liver S9 fraction. The S9 reaction mixtures had a total volume of $1 \mathrm{ml}$, which consisted of $570 \mu \mathrm{l} \mathrm{H}_{2} \mathrm{O}$ (MQ), $200 \mu \mathrm{l} 0.5 \mathrm{M}$ potassium phosphate buffer ( $\mathrm{pH} 7.4$ ), $100 \mu \mathrm{l}$ NADPH regeneration system solution A (BD Bioscience, Breda, The Netherlands), $20 \mu$ NADPH regeneration system solution B (BD Bioscience), $10 \mu \mathrm{l}$ compound stock in DMSO, and $100 \mu \mathrm{l}$ S9 mix (BD Bioscience). After incubating, for respectively 1,6 , and $24 \mathrm{~h}$, the S9-chemical reaction mixtures were heat inactivated $\left(5 \mathrm{~min}\right.$ at $\left.56^{\circ} \mathrm{C}\right)$ and pooled at equal volumes.

\section{Viability and cytotoxicity assays}

WST-1 assay. WST-1 (4-[3-(4-iodophenyl)-2-(4-nitrophenyl)-2H-5-tetrazolio]-1,3-benzene disulfonate, Roche Diagnostic Ned BV, Almere, The Netherlands) is a water-soluble tetrazolium salt, which is cleaved by mitochondrial dehydrogenases to form a colored formazan complex and the amount of formazan correlates to the viability of the cells. Jurkat cells were plated $20 \mathrm{~h}$ in advance in 96 well plates, resulting in about 20,000 cells per well at the starting point of exposure. Exposure was done in 
triplicate in $100 \mu \mathrm{l}$ medium for $24 \mathrm{~h}$ to increasing concentrations of compounds or to the vehicle controls. $10 \mu \mathrm{l}$ of WST-1 reagent was added in the last $2 \mathrm{~h}$ of exposure. Absorbance was measured at $450 \mathrm{~nm}$ in a microplate reader (BioTek, Winooski, VT).

ATPlite assay. ATPlite assay (Perkin Elmer, Oosterhout, The Netherlands) is based on the production of light caused by the reaction of ATP with added luciferase and D-luciferin. The emitted light is proportional to the amount of ATP, which is a marker for cell viability. Plating of the cells and the exposure were done as described above for the WST-1 assay. After exposure, the assay was performed according the manufacturer's protocol.

ToxiLight assay. ToxiLight assay (Lonza, Breda, The Netherlands) is luminescent cytotoxicity assay based on the release of adenylate kinase (AK) from damaged cells. Plating of the cells and the exposure were done as described above for the WST-1 assay. After exposure, the assay was performed according the manufacturer's protocol.

Chemical exposure. Jurkat cells between passages 16-19 were used in the exposure experiment. About 750,000 cells were seeded in $2.7 \mathrm{ml}$ medium per well in 6-well plates. After growing the cells for $20 \mathrm{~h}$, exposure was initiated by adding $0.3 \mathrm{ml}$ medium containing the test compounds (at noncytotoxic concentrations) or vehicle controls. The final DMSO concentration in the medium was kept at $0.1 \%$ $(\mathrm{v} / \mathrm{v})$ for all the samples. The final methanol concentration in the medium was kept at $0.05 \%(\mathrm{v} / \mathrm{v})$ for DMNA. Exposures were performed for $6 \mathrm{~h}$ on three different days with Jurkat cells of different passages. Different batches of cells were used for the exposure experiments with regard to the training and external verification sets of compounds.

Total RNA isolation and cDNA synthesis. After exposure of Jurkat cells for $6 \mathrm{~h}$, the culture medium was removed by centrifugation of the cell suspension ( 5 min at $300 \mathrm{~g}, 4^{\circ} \mathrm{C}$ ). The resulting cells pellet was homogenized in $600 \mu \mathrm{l}$ red cell lysis buffer (RLT) (Qiagen, Venlo, The Netherlands) supplemented with $10 \% \beta$-mercaptoethanol and stored at $-80^{\circ} \mathrm{C}$ until further processing. RNA was isolated with the Qiagen QIAshredder kit according to the manufacturer's protocol. Subsequent RNA purification was performed using the miRNeasy Mini kit (Qiagen) according to the manufacturer's protocol. RNA yield was assessed spectrophotometrically (NanoDrop 2000, Thermoscientific, via Isogen-lifescience, De Meern, The Netherlands). RNA quality was determined by automated gel electrophoresis using BioRad's Experion system (Veenendaal, The Netherlands). Samples with a RNA Quality Index (RQI) number $>8.5$ were considered to be of sufficient quality. cDNA samples were synthesized from the same RNA samples as used for the microarray experiment using miScript Reverse Transcription kit according to the manufacturer's protocol (Qiagen). 
Fluidigm Real -Time PCR verification. Verification of the microarray outcome was performed at ServiceXS (ServiceXS BV, Leiden, The Netherlands) using the 96.96 BioMark Dynamic Array for Real-Time PCR (Fluidigm Corporation, San Francisco, CA), according to the manufacturer's instructions. This platform has the following advantages: (1) it is a high throughput system that allows 9,216 PCR reactions ( $96 \times 96$ ) to be performed on one plate; (2) it is sensitive and reproducible (Jang et al., 2011), without the potential problems related to dye interferences commonly observed in multicolor multiplex qRT-PCR platforms (Morrison, 2010); (3) affordable because of its reaction volume of $6.7 \mathrm{nl}$, which is small as compared to other mRNA expression profiling platforms that are used in biomarker studies, such as Luminex or deep sequencing; (4) it has successfully been applied as high throughput system in large human gene expression studies (Chan et al., 2011; Hollants et al., 2012). In total, three 96.96 BioMark Dynamic Arrays were used for these experiments. The cDNA samples were synthesized from the same RNA samples as used for the microarray experiment using miScript Reverse Transcription kit according to the manufacturer's protocol (Qiagen, Venlo, The Netherlands), and were shipped on dry ice to Service XS in 96-wells plates at a concentration of 10 $\mathrm{ng} / \mathrm{\mu l}$ and a volume of $10 \mu \mathrm{l}$. Before being used on the BioMark array, the cDNA samples were first subjected to 14 cycles of specific target amplification using a $0.2 x$ mixture of all Taqman Gene Expression assays in combination with the Taqman PreAmp Master Mix (Applied Biosystems), followed by $5 \mathrm{x}$ dilution. Water (provided by ServiceXS) was included as no template control (NTC) at the indicated positions. The NTCs were also included in the STA reaction, to serve as a true negative control for the entire procedure. After the $5 x$ dilution, the STA samples were used on a BioMark 96.96 Dynamic Array for Real-Time PCR, for the determination of $\mathrm{Ct}$ values.

Pair-wise combinations of all samples were made with each of the assays in duplicate on the array. The empty assay positions were filled with no assay controls (NAC, in which the 20x assay mix was substituted with water). The default Taqman PCR protocol was used on the BioMark instrument with an annealing temperature of $60^{\circ} \mathrm{C}$ and a total of 35 cycles of PCR.

Analyses of $q R T-P C R$ data. At the end of each PCR cycle, data was collected from the 9,216 reaction chambers on each array, and Ct values were extracted using the BioMark Real-Time PCR analysis software version 3.0.2. The quality threshold was set at 0.65 (default value).

The relative mRNA expression levels were calculated for each individual sample by applying the deltadelta-CT method. Three genes were selected as reference genes, based on the low variability of the relative mRNA expression levels quantified by the microarrays, being beta-2-macroglobulin (B2M, high abundant), Golgi to ER traffic protein 4 (GET4, low abundant), and phosphatidylinositol glycan anchor biosynthesis class $\mathrm{G} 1$ (PIGG). The mRNA expression levels of these genes were not affected (no more than 1.25 -fold) by exposure to the test compounds, Supplementary fig. 1. 
Classifier redundancy analysis. We performed a redundancy analysis on the training set in order to determine the optimal number of classifier genes that are required to accurately distinguish between immunotoxic and nonimmunotoxic compounds. This redundancy analysis started with the 27 classifier genes selected on basis of the microarray data of a set of 31 compounds ( 23 immunotoxic and 8 nonimmunotoxic) that were verified by qRT-PCR. We performed exposures to 13 additional immunotoxic compounds for which the transcriptomic analysis was limited to qRT-PCR on the 27 genes. The expression data of these 44 compounds were taken together to form the training set, in which the redundancy analysis was performed. In this analysis, the criterion for classifying a test compound as direct immunotoxic was when the mRNA expression level of at least one gene was changed by $\geq 2.0$ fold in at least two out of the three independent experiments. In general, for the expression level of the same gene, qRT-PCR gives a higher signal than microarray; therefore, we set the cutoff at twofold. The performance of the identified classifier sets was tested in the external verification set consisting of 17 new immunotoxic and 3 new nonimmunotoxic compounds (Table 1).

Functional interpretation of the 25 classifiers. The major gene ontology (GO) processes and molecular functions in which the 25 classifiers are involved were obtained from $G O$ (http://www.geneontology.org/) and GeneCards (http://www.genecards.org/) (Shao et al., 2013), and were categorized into eight functional themes (Table 6). The visualization of the effects of each compound on these functional themes was performed using ToxPi software from the US EPA (Reif et al., 2013). The mRNA levels of the 25 classifier genes of the 44 compounds from the training set were used as the input for ToxPi software. ToxPi calculates a ToxPi score for each compound on each functional theme and rank the compounds by their overall ToxPi scores. The ToxPi score (between 0 to 1 ) is calculated by dividing the results of each chemical on the genes that are involved in this functional theme by the maximum results on the same genes from the 44 compounds. 


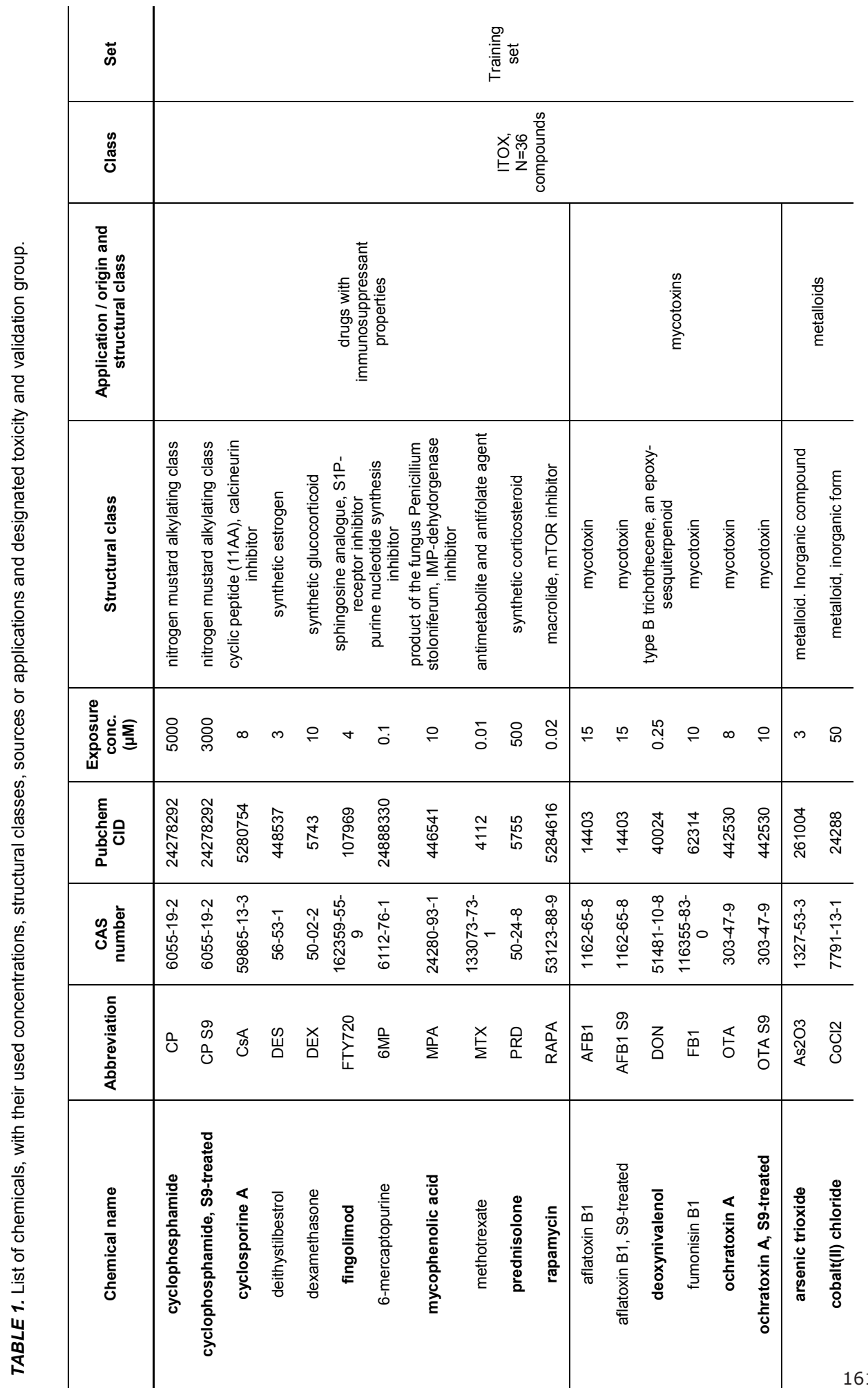




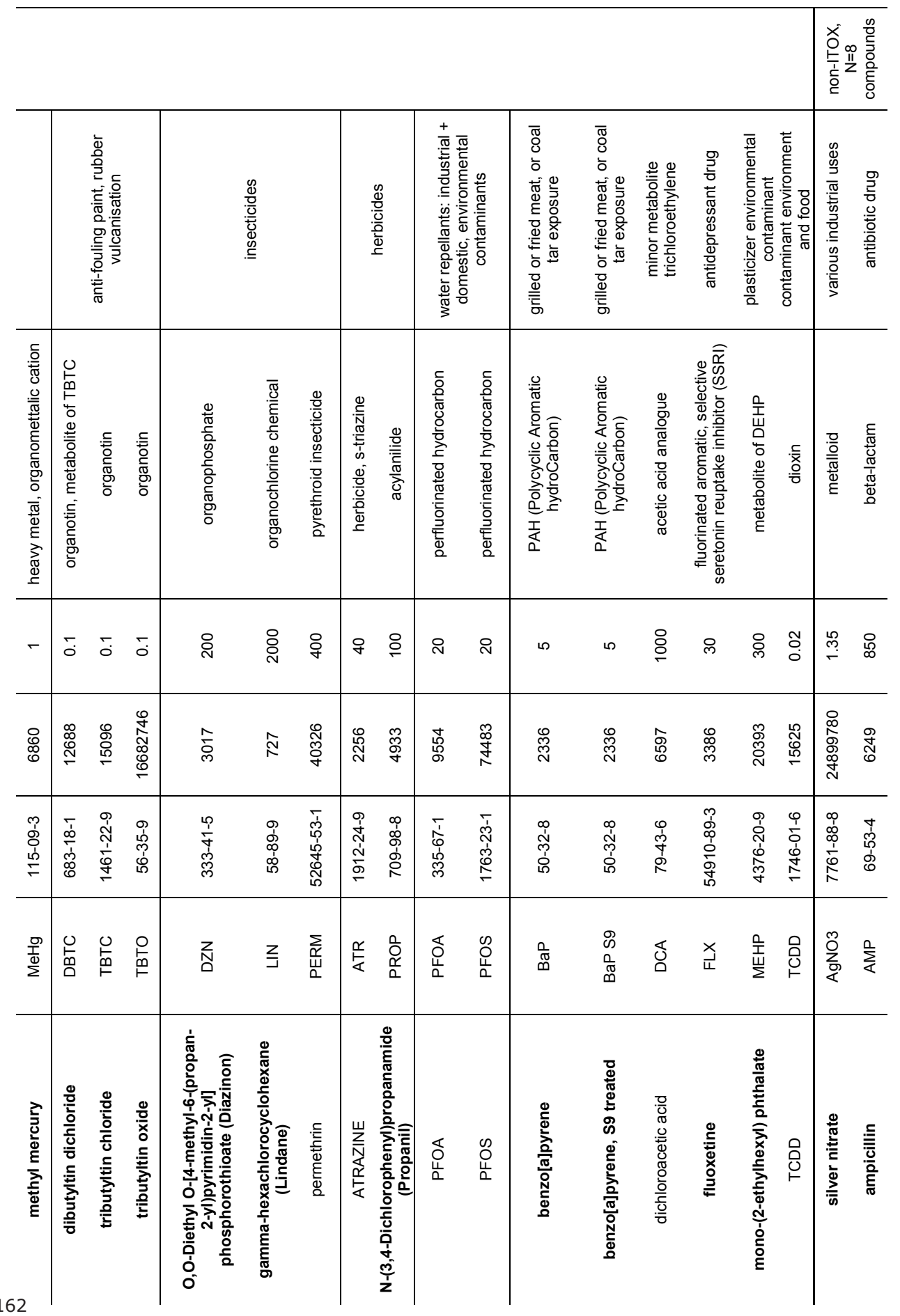




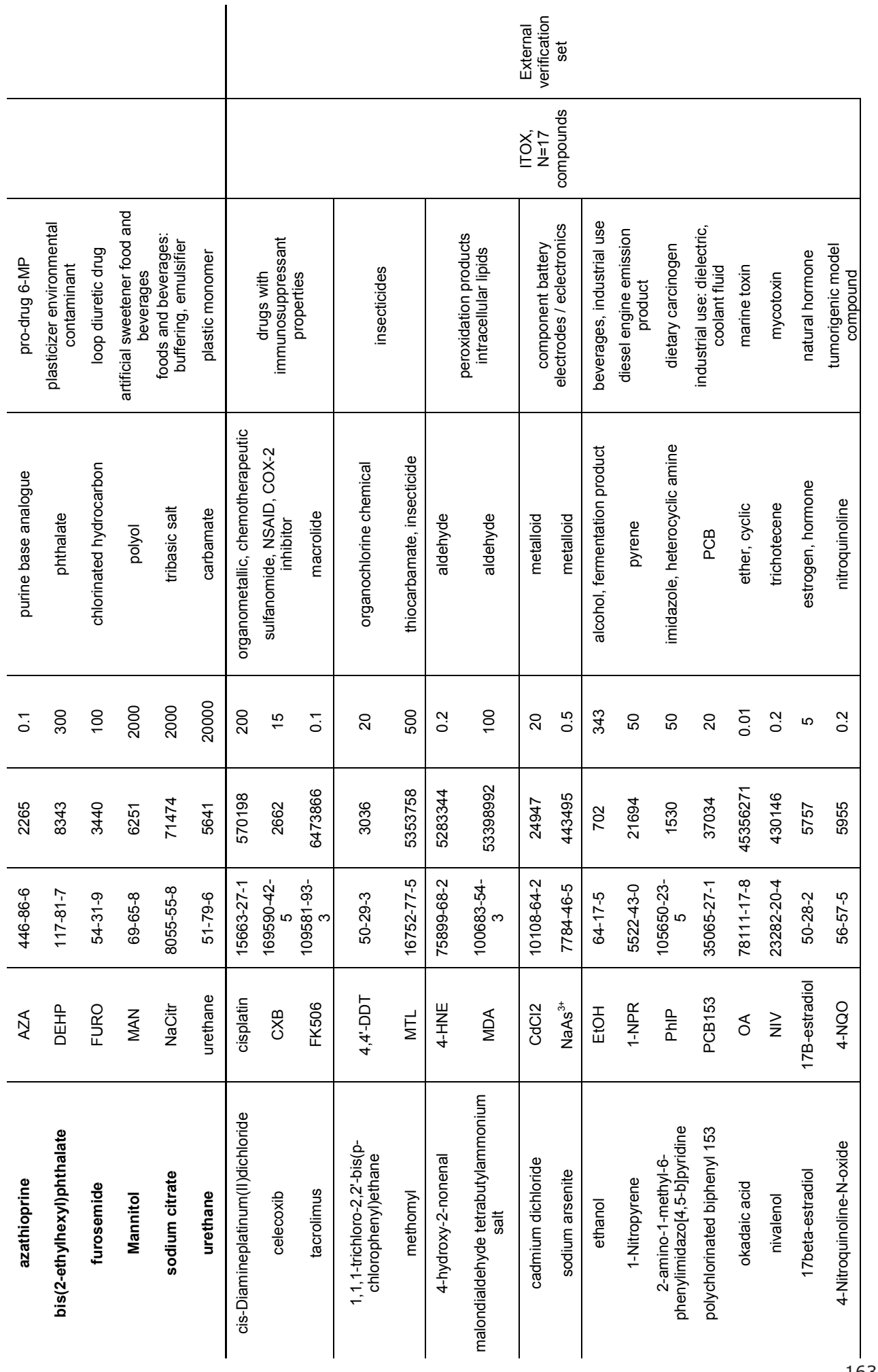




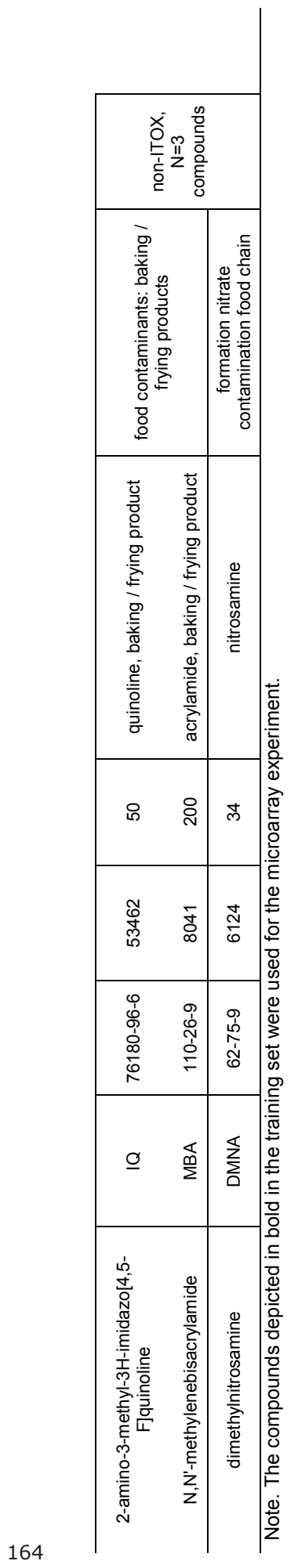




\section{Results}

\section{Selection of test compounds}

Two sets of compounds were constructed, a training set for the identification of the classifiers and an external verification set for determining the performance of the selected classifiers.

For the training set, 44 test compounds were selected for assessing their effects on gene expression of the human Jurkat $T$ cell line in vitro. These test compounds comprised direct immunotoxic chemicals, immunosuppressive drugs with known modes of action, and nonimmunotoxic control compounds (Table 1). The training set consisted of the 31 compounds (23 immunotoxic and 8 nonimmunotoxic) that were previously used in a Jurkat transcriptome study (Shao et al., 2013) and 13 additional immunotoxicants. Aflatoxin B1, benzo[a]pyrene, cyclophosphamide, and ochratoxin A were bioactivated using human liver S9 fraction. For these four compounds, both the parent compounds and the bioactivated samples were included in the study. The training set was used for the selection of the candidate classifiers.

In order to verify the statistical power of the identified classifiers, we further selected another set of 20 compounds, being the external verification set, which consisted of 17 immunotoxic and 3 nonimmunotoxic compounds

\section{Exposure of Jurkat cells to the test compounds}

The effect of the test compounds on gene expression in Jurkat cells was assessed upon $6 \mathrm{~h}$ of exposure to subcytotoxic concentrations of the test compounds (Table 1). Subcytotoxic concentrations were defined as the concentrations that led to $<20 \%$ decrease of viability (CV80) and $<20 \%$ induction of cytotoxicity after $24 \mathrm{~h}$ of exposure. Viability was first assessed by the WST-1 and ATPLite assays (Roche and PerkinElmer, respectively). Dose-response curves were made for each of the test compounds. Thereafter, cytotoxicity was assessed for the selected doses by the Toxilight assay (Lonza), which showed that none of the selected doses induced cytotoxicity, see Supplementary table 1 for results.

\section{Identification and external verification of candidate classifier genes}

In the microarray study, genes were considered to be significantly affected when upregulated $\geq 1.62$ fold (2log 0.7 ) or downregulated $\leq 1.62(2 \log -0.7)$ in at least three of the four biological replicates as compared to the average of the vehicle controls. Based on these criteria, 1,445 genes were affected by at least one compound in the microarray experiment (Shao et al., 2013). From these 1,445 genes, 93 genes were further selected, and 80 of them were affected by at least three compounds, and the other 13 genes were affected by either one single compound or compound class (Supplementary table 
2). The majority of these 93 genes are associated with human immune-related diseases (Supplementary table 3). We subjected these genes to unsupervised hierarchical clustering analysis thereby identifying 18 clusters (A-R) of co-regulated genes (Fig. 1). Out of these 93 genes, we selected 28 genes based on the largest up- or downregulation in response to the immunotoxicants for qRT-PCR verification. These 28 genes covered 16 out of 18 clusters. qRT-PCR was used to determine the expression levels of the 28 genes in Jurkat cells exposed to the 44 compounds of the training set. With respect to the 31 compounds already examined in the previous transcriptome study, qRT-PCR-based expression patterns correlated well with the microarray data for 27 out of 28 genes $(R \geq 0.69)$ (Shao et al., 2013). NANOS1 was the only exception $(R=0.14)$ and was excluded from further analyses (See Table 2 for the list of 27 genes). The qRT-PCR data of the 27 candidate classifier genes for the 44 compounds of the training set are shown in Table 3 . Then, the expression levels of the 27 candidate classifier genes were tested by qRT-PCR on the external verification set of 20 compounds. The results of this qRT-PCR experiment can be found in Table 4 .

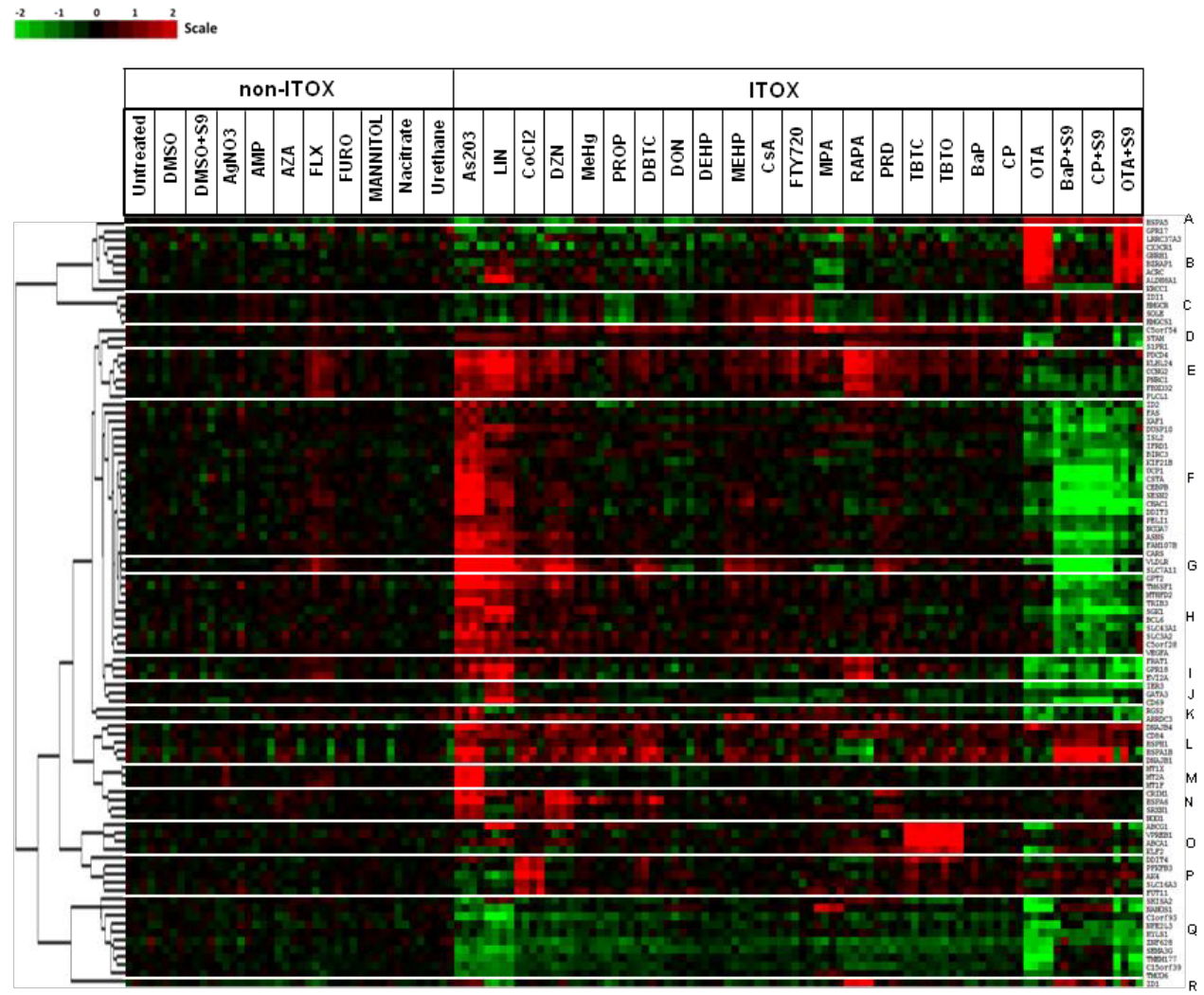

FIG. 1. Unsupervised hierarchical clustering of 93 genes. The heat map visualizes the microarray- based $m R N A$ expression patterns of 93 candidate genes in Jurkat cells upon $6 \mathrm{~h}$ exposures to subcytotoxic levels of 31 different 
immunotoxic and nonimmunotoxic chemicals and drugs. Microarray data of 93 genes are given for four independent experiments. These genes were selected by applying the following criteria: $\geq 1.62$-fold regulation (2log intensity ratio over carrier control $\geq 0.7$ or $\leq-0.7$ ) in at least three of the four biological replicates. Among these 93 genes, 80 genes were regulated by at least three compounds, and the other 13 genes were specifically regulated by a single immunotoxic chemical/ chemical class. Eighteen sub-clusters of co-regulated genes can be distinguished, as listed from $A$ to $R$. Green represents down regulation, black no regulation, and red up regulation. The colour intensity related to the 2log ratio is indicated by the bar. The 28 candidate classifier genes covered 16 out of these 18 sub-clusters. We did not select candidate genes from clusters $D$ and $\mathrm{J}$ because the genes in these clusters had similar expression patterns to clusters $E$ and I, respectively. For the abbreviations of all the compounds, see Table 1.

\section{Classifier redundancy analysis}

Redundancy analysis was performed on the data of the training set starting with the 27 genes (Fig. 2). We observed that the true negative rate (specificity) of the classifier set can be increased from 63 to $88 \%$ if the number of classifiers is reduced to 25 , whereas the true positive rate (sensitivity) of the classifier set still remains the same. This is because that MT1F and GPR17, the two genes that were deleted from the classifier set, each generated one false-negative outcome, being $\mathrm{AgNO}_{3}$ and Mannitol, respectively. We also observed that the same sensitivity and specificity can be achieved even if the number of classifiers is reduced to 7. These seven genes are GPR18, VPREB1, HMGCS1, ABCA1, CHAC1, ALDH8A1, and KLF2.

The performance of the set of 25 and seven classifier genes in the external verification set is shown in Table 5 . The 25 classifier gene set gave a very good performance showing an accuracy, sensitivity, and specificity of 85,88 , and $67 \%$, respectively. In total, three compounds were misclassified, being one false positive compound (2-amino-3-methyl-3H-imidazo[4,5-F]quinolone) and two false-negative compounds (17beta-estradiol and okadaic acid). The seven classifier gene set gave an accuracy, sensitivity, and specificity of 75,76 , and $67 \%$, respectively. The lower accuracy and sensitivity for the seven classifier gene set is caused by two additional false negative outcomes, being 4-hydroxy-2nonenal and sodium arsenite. 
TABLE 2. List of 27 genes that were verified by $q R T-P C R$ experiment.

\begin{tabular}{|c|c|c|c|c|}
\hline $\begin{array}{l}\text { Gene } \\
\text { symbol }\end{array}$ & Description & $\begin{array}{l}\text { mRNA } \\
\text { accession }\end{array}$ & Taqman assay & $\begin{array}{l}\text { classifier gene } \\
\text { sets }\end{array}$ \\
\hline ABCA1 & ATP-binding cassette, sub-family A (ABC1), member 1 & NM_005502 & Hs01059118_m1 & \multirow{25}{*}{$\begin{array}{l}\mathrm{N}=25 \text { classifier } \\
\text { genes }\end{array}$} \\
\hline ABCG1 & $\begin{array}{c}\text { ATP-binding cassette, sub-family G (WHITE), member } \\
1\end{array}$ & NM_004915 & Hs00245154_m1 & \\
\hline AK4 & adenylate kinase 4 & NM_001005353 & Hs03405743_g1 & \\
\hline ALDH8A1 & aldehyde dehydrogenase 8 family, member $\mathrm{A} 1$ & NM_001193480 & Hs00988965_m1 & \\
\hline ARRDC3 & arrestin domain containing 3 & NM_020801 & Hs00385845_m1 & \\
\hline BZRAP1 & benzodiazapine receptor (peripheral) associated protein & NM_004758 & Hs00270490_m1 & \\
\hline CCNG2 & cyclin G2 & NM_004354 & Hs00171119_m1 & \\
\hline CEBPB & CCAAT/enhancer binding protein (C/EBP), beta & NM_005194 & Hs00270923_s1 & \\
\hline $\mathrm{CHAC1}$ & ChaC, cation transport regulator homolog 1 (E. coli) & NM_001142776 & Hs00225520_m1 & \\
\hline CRIM1 & $\begin{array}{c}\text { cysteine rich transmembrane BMP regulator } 1 \text { (chordin- } \\
\text { like) }\end{array}$ & NM_016441 & Hs00212750_m1 & \\
\hline FBXO32 & F-box protein 32 & NM_001242463 & Hs01041408_m1 & \\
\hline GPR18 & G protein-coupled receptor 18 & NM_001098200 & Hs00245542_m1 & \\
\hline HMGCS1 & 3-hydroxy-3-methylglutaryl-CoA synthase 1 (soluble) & NM_001098272 & Hs00940429_m1 & \\
\hline HSPA1B & heat shock $70 \mathrm{kDa}$ protein $1 \mathrm{~B}$ & NM_005346 & $\mathrm{Hs} 01040501-\mathrm{sH}$ & \\
\hline HSPA5 & $\begin{array}{l}\text { heat shock } 70 \mathrm{kDa} \text { protein } 5 \text { (glucose-regulated protein, } \\
78 \mathrm{kDa})\end{array}$ & NM_005347 & Hs00946084_g1 & \\
\hline ID1 & $\begin{array}{c}\text { inhibitor of DNA binding 1, dominant negative helix- } \\
\text { loop-helix protein }\end{array}$ & NM_002165 & Hs00357821_g1 & \\
\hline ID2 & $\begin{array}{l}\text { inhibitor of DNA binding 2, dominant negative helix- } \\
\text { loop-helix protein }\end{array}$ & NM_002166 & Hs00747379_m1 & \\
\hline KLF2 & Kruppel-like factor 2 (lung) & NM_016270 & Hs00360439_g1 & \\
\hline KLHL24 & kelch-like 24 (Drosophila) & NM_017644 & Hs00214210_m1 & \\
\hline NQO1 & $\mathrm{NAD}(\mathrm{P}) \mathrm{H}$ dehydrogenase, quinone 1 & NM_000903 & Hs01045995_m1 & \\
\hline SLC7A11 & $\begin{array}{l}\text { solute carrier family } 7 \text { (anionic amino acid transporter } \\
\text { light chain, xc- system), member } 11\end{array}$ & NM_014331 & Hs00921938_m1 & \\
\hline SRXN1 & sulfiredoxin 1 & NM_080725 & Hs00607800_m1 & \\
\hline TM6SF1 & transmembrane 6 superfamily member 1 & NM_001144903 & Hs00224823_m1 & \\
\hline VLDLR & very low density lipoprotein receptor & NM_001018056 & Hs01047538_m1 & \\
\hline VPREB1 & pre-B lymphocyte 1 & NM_007128 & Hs00356766_g1 & \\
\hline GPR17 & $\begin{array}{c}\text { G protein-coupled receptor } 17 \\
\text { metallothionein } 1 \mathrm{~F}\end{array}$ & $\begin{array}{c}\text { NM_001161415 } \\
\text { NM_005949 }\end{array}$ & $\begin{array}{l}\text { Hs00171137_m1 } \\
\text { Hs00744661_sH }\end{array}$ & not shortlisted \\
\hline
\end{tabular}




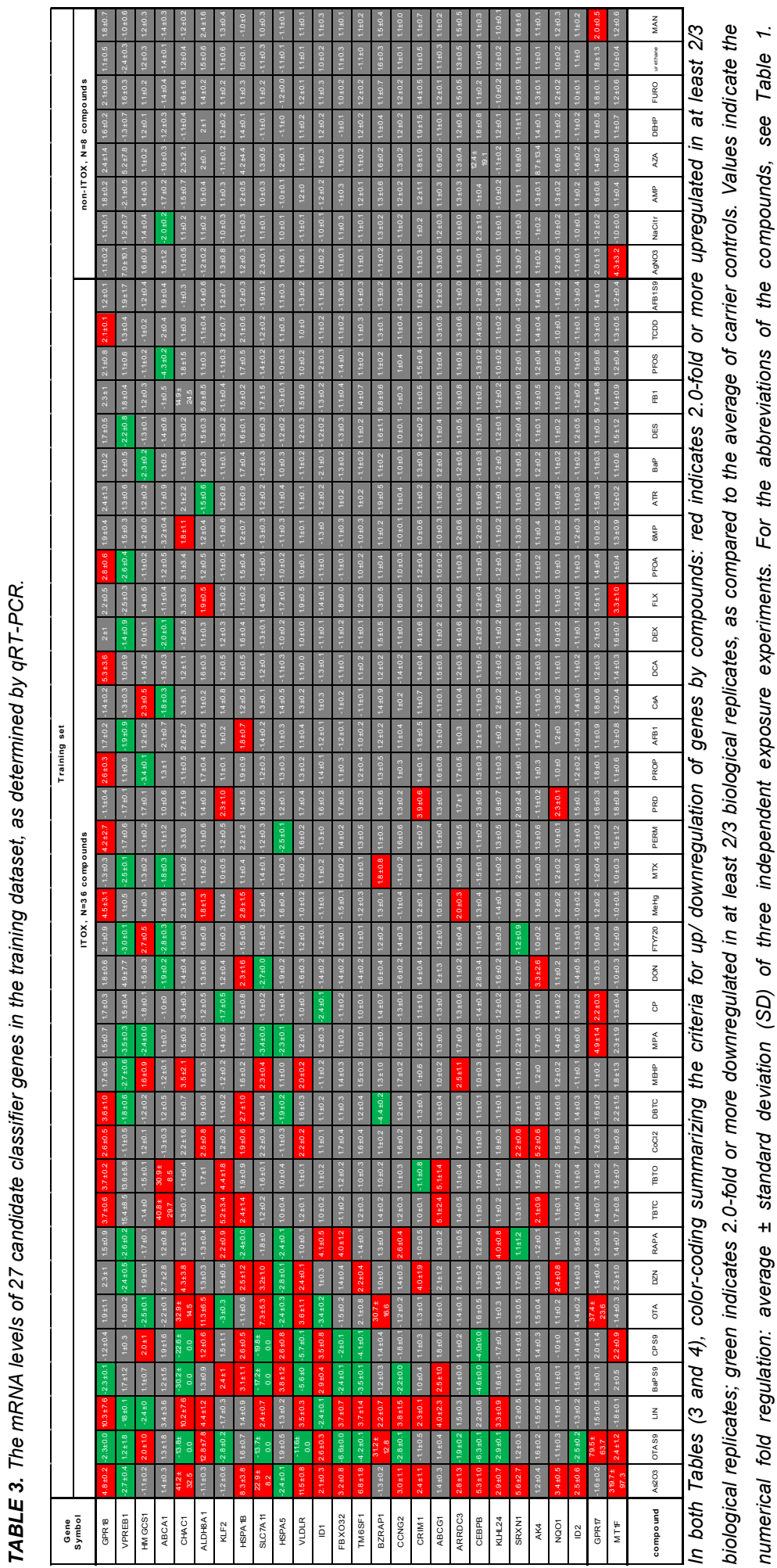


TABLE 4. The mRNA levels of 27 candidate classifier genes in the external verification dataset, as determined by qRT-PCR.

\begin{tabular}{|c|c|c|c|c|c|c|c|c|c|c|c|c|c|c|c|c|c|c|c|c|c|}
\hline \multirow{2}{*}{$\begin{array}{c}\text { classifier } \\
\text { set }\end{array}$} & \multirow{2}{*}{$\begin{array}{l}\text { Gene } \\
\text { Symbol }\end{array}$} & \multicolumn{20}{|c|}{ external verification set } \\
\hline & & \multicolumn{17}{|c|}{ ITOX $\mathrm{N}=17$ compounds } & \multicolumn{3}{|c|}{ non-ITOX: $N=3$ compounds } \\
\hline \multirow{25}{*}{ Yes } & GPR 18 & $3.1 \pm 4.7$ & $2.2 \pm 1.5$ & $2.3 \pm 2$ & $-1.4 \pm 1.9$ & $1.0 \pm 1.6$ & $1.6 \pm 1.8$ & $3.0 \pm 2.4$ & $2.0 \pm 1.7$ & $1.4 \pm 1.2$ & $2.6 \pm 2.3$ & $1.2 \pm 1.0$ & $1.6 \pm 1.6$ & $1.1 \pm 1.3$ & $1.7 \pm 3.1$ & $1.0 \pm 1.1$ & $1.1 \pm 1.0$ & $1.1 \pm 1.2$ & $1.0 \pm 1.0$ & $1.1 \pm 1.0$ & $1.0 \pm 2.2$ \\
\hline & VPREB 1 & $-3.2 \pm 1.4$ & $1.1 \pm 1.2$ & $-1.9 \pm 1.5$ & $-2.4 \pm 1.5$ & $-3.2 \pm 2.2$ & $-4.3 \pm 4$ & $-2.5 \pm 1.6$ & $1.0 \pm 1.8$ & $-1.3 \pm 2.1$ & $-2.5 \pm 1.9$ & $-2.2 \pm 1.5$ & $-1.1 \pm 1.5$ & $-2.4 \pm 1.4$ & $-2.2 \pm 1.3$ & $-4.1 \pm 3.3$ & $-1.9 \pm 1.6$ & $-2.9 \pm 2.4$ & $1.1 \pm 1.3$ & $-2.3 \pm 1.1$ & $-1.8 \pm 1.5$ \\
\hline & HMGCS1 & $-1.5 \pm 1.1$ & $2.2 \pm 1.3$ & $1.0 \pm 1.2$ & $-1.1 \pm 1.1$ & $1.8 \pm 1.1$ & $2.1 \pm 1.2$ & $2.0 \pm 1.2$ & $-1.2 \pm 1.1$ & $1.3 \pm 1.2$ & $-1.1 \pm 1.1$ & $-1.4 \pm 1.1$ & $-2.4 \pm 1.1$ & $-1.1 \pm 1.2$ & $-1.1 \pm 1.3$ & $1.1 \pm 1.2$ & $-1,1 \pm 1$ & $-1.1 \pm 1.1$ & $1.2 \pm 1.1$ & $-1.3 \pm 1.1$ & $1.0 \pm 1.1$ \\
\hline & ABCA 1 & $1.2 \pm 1.0$ & $13 \pm 1.3$ & $11 \pm 1.7$ & $-1.4 \pm 2.1$ & $-1.6 \pm 1.3$ & $-2+6.6$ & $1.1 \pm 1.4$ & $1.2 \pm 1.6$ & $1.5 \pm 1.3$ & $-1.1 \pm 1.7$ & $-2.2 \pm 2.5$ & $13 \pm 1.2$ & $-2.7 \pm$ & $-18 \pm$ & $-1.3 \pm 1.2$ & $10 \pm 2.0$ & $-2.1 \pm$ & $-18 \pm$ & $1.0 \pm 2.2$ & $-2.3 \pm 2.1$ \\
\hline & CHAC1 & $34.3 \pm 2.3$ & $70 \pm 1.5$ & $-1.5 \pm 2.5$ & $1.1 \pm 1.9$ & $1.7 \pm 2.5$ & $-1.2 \pm 1.9$ & $1.2 \pm 3$ & $2 \pm 2.4$ & $2.1 \pm 3.1$ & $19 \pm 2.5$ & $-1.1 \pm 2.4$ & $-1.2 \pm 1.4$ & $1.4 \pm 1.7$ & $-1.2 \pm 1.2$ & $1 \pm 3.6$ & $1.5 \pm 3$ & $14 \pm 2$ & $-1.5 \pm 1.5$ & $-1.1 \pm 2.2$ & $1.5 \pm 2.1$ \\
\hline & ALDHBA1 & $-1.4 \pm 1.9$ & $2.5 \pm 1.6$ & $-1.5 \pm 1.4$ & $-1.4 \pm 1.7$ & $1.4 \pm 1.4$ & $-1.6 \pm 1.7$ & $-1.3 \pm 1.3$ & $1.4 \pm 1.8$ & $13 \pm 2$ & $-1.3 \pm 1.6$ & $13 \pm 1.3$ & $1.2 \pm 1.7$ & $-19 \pm 13$ & $1.2 \pm 1.2$ & $1.4 \pm 2.1$ & $-1.2 \pm 1.7$ & $-1.3 \pm 1.5$ & $1 \pm 1.1$ & $1.1 \pm 1.7$ & $11+2.1$ \\
\hline & $\mathrm{KLF} 2$ & $1.1 \pm 1.3$ & $1.3 \pm 1.3$ & $-1.1 \pm 1.7$ & $-1.2+14$ & $-1.3 \pm 1.1$ & $1.0 \pm 1.2$ & $-1.6 \pm 1.4 \mid$ & $1.8 \pm 2.2$ & $1.1 \pm 3.2$ & $-1.2 \pm 1.3$ & $-1.2 \pm 1.4$ & $1.0 \pm 1.7$ & $-1.5 \pm 1.4$ & $-1.4 \pm 1.3$ & $-1.8+1.5$ & $-1.5 \pm 1.1$ & $1.1 \pm 1.4$ & $1.1 \pm 1.2$ & $1.2 \pm 1.4$ & $-13 \pm 1.6$ \\
\hline & HSPA $1 B$ & $2.0 \pm 1.4$ & $4.3 \pm 1.4$ & $-2.5 \pm 1.7$ & $-1.2 \pm 1.6$ & $-1.3 \pm 1.6$ & $-1.3 \pm 1.3$ & $-1.5 \pm 1.5$ & $1.7 \pm 1.6$ & $1.8 \pm 2.6$ & $-1.2 \pm 1.4$ & $-1.8 \pm 1.5$ & $0.1 \pm 1.5$ & $-1.5 \pm 1.2$ & $-1.5 \pm 1.2$ & $-1.3 \pm 1.7$ & $-1.2 \pm 1.3$ & $-1.2 \pm 1.5$ & $-1.7 \pm 1.1$ & $-2 \pm 1.7$ & $-1.3 \pm 1.7$ \\
\hline & SLC7A 11 & $14.1 \pm 1.3$ & $9.5 \pm 1.1$ & $-1.8 \pm 1.2$ & $1.1 \pm 1.2$ & $3.7 \pm 1.0$ & $-1.1 \pm 1.3$ & $1.2 \pm 1.1$ & $1.5 \pm 1.9$ & $2.8 \pm 2.1$ & $1.7 \pm 1.2$ & $1.1 \pm 1.2$ & $-111 \pm 1.5$ & 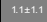 & $1.3 \pm 1.4$ & $1.2 \pm 1.1$ & $1.2 \pm 1.4$ & $-1.1 \pm 1.1$ & $1.2 \pm 1.3$ & $-1.1 \pm 1.2$ & $1.7 \pm 1.3$ \\
\hline & HSPA5 & 1.1.11.2. & $1.7 \pm 1.2$ & $-1.4 \pm 1.1$ & $1.0 \pm 1.1$ & $1.4 \pm 1.2$ & 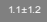 & $-1.2 \pm 1$ & $1.0 \pm 1.2$ & $1.3 \pm 1.1$ & $-1.2 \pm 1.3$ & $1.1 \pm 1.1$ & $-1.1 \pm 1.2$ & $-1.1 \pm 1.4$ & $-1.3 \pm 1.6$ & $1.0 \pm 1.3$ & $1.5 \pm 1.2$ & $1 \pm 1.2$ & $1.2 \pm 1.1$ & $1.0 \pm 1.2$ & $1.1 \pm 1.3$ \\
\hline & VLDLR & $7.0 \pm 1.2$ & $3.7 \pm 1.2$ & $-1.3 \pm 1.1$ & 1.111.1. & $1.4 \pm 1.2$ & $1.0 \pm 1.3$ & $1.1 \pm 1$ & 1.1.11.11 & $1.2 \pm 1.2$ & $1.3 \pm 1.1$ & $1.1 \pm 1.1$ & $1 \pm 1.2$ & $1.1 \pm 1.2$ & $1.1 \pm 1.2$ & $1.2 \pm 1.1$ & $1.2 \pm 1.1$ & $1.0 \pm 1.2$ & $1.0 \pm 1.0$ & 1.1.1 & $1.4 \pm 1.1$ \\
\hline & ID1 & $1.6 \pm 1.1$ & $2.0 \pm 1.2$ & $-1.2 \pm 1.1$ & $-1.2 \pm 1.1$ & $-2.3 \pm 1.2$ & $2.1 \pm 2.5$ & $-1.1 \pm 1.1$ & $2.1 \pm 2.6$ & $1.2 \pm 1.7$ & $-1.6 \pm 1$ & $-1.1 \pm 1.1$ & $1.0 \pm 1.1$ & $1.1 \pm 1.0$ & $1 \pm 1.2$ & $-1.1 \pm 1.1$ & $1.0 \pm 1.1$ & $1.0 \pm 1.1$ & $1.1 \pm 1.3$ & $-1.1 \pm 1.2$ & $1.2 \pm 1.1$ \\
\hline & $\mathrm{FBXO} 22$ & $3.9 \pm 1.2$ & $1.2 \pm 1.2$ & $1.0 \pm 1.2$ & 1. $1 \pm 1.3$ & $1.2 \pm 1.4$ & $1.0 \pm 1.3$ & $1.2 \pm 1.2$ & $1.3 \pm 1.1$ & $1.3 \pm 1.5$ & $1.4 \pm 1.2$ & $1.2 \pm 1.2$ & $1.2 \pm 1$ & $1.3 \pm 1.1$ & $1.4 \pm 1.1$ & $1.1 \pm 1.4$ & $1.1 \pm 1.2$ & $1.2 \pm 1.3$ & $1.2 \pm 1.2$ & $-1.1 \pm 1.2$ & $1.0 \pm 1.1$ \\
\hline & TM6SF1 & $4.3 \pm 1.4$ & $18 \pm 12$ & $1.0 \pm 1.1$ & $1.1 \pm 1.1$ & $1.5 \pm 1.2$ & $1.0 \pm 1.2$ & $1.1 \pm 1.1$ & $1.4 \pm 1.5$ & $1.4 \pm 1.4$ & $1.4 \pm 1.1$ & $1.1 \pm 1.0$ & $1.1 \pm 1.1$ & $1.1 \pm 1.2$ & $1.2 \pm 1.2$ & $1.0 \pm 1.1$ & $1.1 \pm 1.1$ & $1.1 \pm 1.1$ & $1.1 \pm 1.1$ & $1 \pm 1.1$ & $1 \pm 1.2$ \\
\hline & BZRAP1 & $3.7 \pm 1.4$ & $-1.1 \pm 16$ & $-1.5 \pm 1.5$ & $-1.6 \pm 1.5$ & $-1.4 \pm 1$ & $-1.5 \pm 1.6$ & $-1.8 \pm 1.8$ & $1 . \pm 1.2$ & $-2.1 \pm 1.2$ & $-2.2 \pm 1.4$ & $-1.5 \pm 1.6$ & $-1.5 \pm 1.8$ & $-1.5 \pm 1.3$ & $-1.7 \pm 2$ & $-1.6 \pm 1.3$ & $-13 \pm 17$ & $-1.9+2.2$ & $1.0 \pm 1.4$ & $-1.6 \pm 1.3$ & $-1.6 \pm 1.4$ \\
\hline & $\mathrm{CCNG} 2$ & $2.4 \pm 1.2$ & $1.1 \pm 1.1$ & $-1.3 \pm 1.1$ & $-1.1 \pm 1.0$ & $1.0 \pm 1.1$ & $-1.1 \pm 1.2$ & $1.2 \pm 1$ & $1.1 \pm 1.6$ & $1.3 \pm 1.5$ & $1.2 \pm 1.1$ & $1.0 \pm 1.1$ & $10 \pm 1.1$ & $-1.1 \pm 1.3$ & $1.2 \pm 1.2$ & $1.0 \pm 1.2$ & $-1.1 \pm 1.1$ & $1.0 \pm 1.1$ & $1.0 \pm 1.3$ & $-1.2 \pm 1.1$ & $-1.2 \pm 11$ \\
\hline & CRIM 1 & $-1.2 \pm 1.2$ & $4.3 \pm 2.0$ & $1 \pm 1.9$ & $-12 \neq 2.2$ & $17 \pm 1.7$ & $1.4 \pm 1.4$ & $-1.2+2.2$ & $1.3 \pm 1.7$ & $1.5 \pm 1.8$ & $1.0 \pm 1.1$ & $-1.7 \pm 2.5$ & $13 \pm 1.6$ & $1.3 \pm 1.8$ & $1 \pm 2.2$ & $1.1 \pm 1.9$ & $-12 \pm 2.1$ & $1.2 \pm 1.7$ & $-1.2 \neq 1.5$ & $1.1 \pm 1.8$ & $11 \pm 1.9$ \\
\hline & ABCG1 & $1.2 \pm 1.0$ & $-1.1 \pm 1.5$ & $-1.3 \pm 1.4$ & $-1.1 \pm 1.13$ & $-1.4 \pm 1.5$ & $-1.5 \pm 1.1$ & $-1.6 \pm 1.3$ & $1.4 \pm 1.7$ & $1.1 \pm 2.6$ & $-1.5 \pm 1.3$ & $-1.2 \pm 1.3$ & $13 \pm 1.5$ & $-1.5 \pm 1.2$ & $-1.3 \pm 12$ & $-1.5 \pm 1.2$ & $-1.2 \pm 1.2$ & $-1.1 \pm 1.4$ & $-1.1 \pm 1.3$ & $-12 \pm 1.4$ & $-13 \pm 1.5$ \\
\hline & ARRDC3 & $12.5 \pm 1.7$ & $2 \pm 1.3$ & $1.2 \pm 1.7$ & $-1.3 \pm 1.6$ & $1.5 \pm 1.3$ & $1.2 \pm 1.5$ & $1.4 \pm 1.6$ & $1.3 \pm 1.5$ & $1.3 \pm 1$ & $1.3 \pm 1.4$ & $1.1 \pm 1.5$ & $1.2 \pm 1.3$ & $-1.1 \pm 1.4$ & $1.1 \pm 2.2$ & $1.0 \pm 1.2$ & $1.2 \pm 1$ & $1.0 \pm 1.4$ & $1.1 \pm 1.2$ & $1.0 \pm 1.2$ & $1.3 \pm 1.4$ \\
\hline & CEBPB & $4.0 \pm 1.3$ & $1.4 \pm 1.6$ & $-1.5 \pm 1.7$ & $-1.2 \pm 1.8$ & $1.0 \pm 1.8$ & $-1.6 \pm 1.4$ & $-1.1 \pm 1.8$ & $1.6 \pm 1.6$ & $1.1 \pm 2.4$ & $-1.3 \pm 1.5$ & $-1.4 \pm 1.3$ & $1.3 \pm 1.5$ & $-1.1 \pm 1.8$ & $-1.1 \pm 1.4$ & $-1.1 \pm 1.7$ & $1 \pm 2.1$ & $1.1 \pm 1.9$ & $-1.3 \pm 1.7$ & $1.0 \pm 1.6$ & $-1.2 \pm 1.8$ \\
\hline & KLHL24 & $2.6 \pm 1.1$ & $1.6 \pm 1.1$ & $-1.3 \pm 1.2$ & $1.2 \pm 1.0$ & $1.3 \pm 1.1$ & $-1.2 \pm 1.4$ & $1.3 \pm 1.1$ & t.1 11.5 & $1.5 \pm 1.4$ & $1.3 \pm 1.1$ & 1.1111.11 & $1.2 \pm 1.3$ & $1.0 \pm 1.4$ & $1.2 \pm 1.2$ & $1.3 \pm 1.1$ & $1.1 \pm 1.2$ & $1.0 \pm 1.4$ & 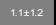 & $1.2 \pm 1.2$ & $1.1 \pm 1.4$ \\
\hline & SRXN1 & $1.9 \pm 2.1$ & $2.9 \pm 2.1$ & $-1.2 \pm 2.8$ & $-1.7 \pm 1.5$ & $1.6 \pm 2$ & $-2.2 \pm 1.5$ & $-1.6 \pm 17$ & $2.3 \pm 3.0$ & $1.2 \pm 4.5$ & $-1.7 \pm 1.8$ & $-1.7 \pm 2$ & $-1.2 \pm 1.8$ & $-1.2 \pm 1.7$ & $-1.3 \pm 1.6$ & $-1.4 \pm 1.7$ & $1 \pm 2.2$ & $-1.2 \pm 1.4$ & $1.3 \pm 1.6$ & $-1.3 \pm 2.4$ & $-1.4 \pm 1.6$ \\
\hline & AK4 & $1.0 \pm 1.2$ & $1.2 \pm 1.4$ & $1.0 \pm 1.3$ & $-1.2 \pm 1.1$ & $1.0 \pm 1.2$ & $.0 \pm 1.1$ & $1.0 \pm 1.2$ & $1.2+1.2$ & 1.11+1.5 & $-1.2 \pm 1.0$ & $-1.1 \pm 1.1$ & $1.0 \pm 1.2$ & $-1.2 \pm 1.1$ & $-1.1 \pm 1.1$ & $-1.1 \pm 1.2$ & $1.0 \pm 1.2$ & $-1.1 \pm 1.1$ & $1.0 \pm 1.1$ & $-1.1 \pm 1.1$ & $1.1 \pm 1.1$ \\
\hline & NQO1 & $1.2 \pm 1.1$ & $2.9 \pm 1.1$. & $1.0 \pm 1.2$ & $1.1 \pm 1.1$ & $1.6 \pm 1.0$ & 1.1.1.1.1. & $1.0 \pm 1.2$ & $1.8+1.5$ & $1.6 \pm 2$ & $1.0 \pm 1.2$ & $-1.2 \pm 1.1$ & $1.1 \pm 1.0$ & $1.0 \pm 1.2$ & $1.3 \pm 1.2$ & 1.1.11.1.1 & $1.0 \pm 1.0$ & $1.0 \pm 1.1$. & $1.0 \pm 1.3$ & $-1.1 \pm 1.0$ & $1.4 \pm 1.1$ \\
\hline & ID2 & $18 \pm 14$ & $2.4 \pm 1.1$ & $10 \pm 12$ & $-1.1 \pm 1.1$ & $13 \pm 1.2$ & $1.2 \pm 1.3$ & $1.0 \pm 1.2$ & $-1.1 \pm 1.1$ & $1.0 \pm 1.0$ & $-1.1 \pm 1.0$ & $1.0 \pm 1.0$ & $1.2 \pm 1.2$ & $1.0 \pm 1.0$ & $1.0 \pm 11$ & $1.1 \pm 1.2$ & $1.2 \pm 1.2$ & $1.0 \pm 1.2$ & $12 \pm 1.2$ & $1.0 \pm 1.2$ & $1.4 \pm 1.2$ \\
\hline \multirow{2}{*}{$\mathrm{N}$} & GPR 17 & $8.8 \pm 1.3$ & $-1.5 \pm 1.4$ & $1.3 \pm 1.3$ & $-1.3 \pm 1.3$ & $-1.2 \pm 1.3$ & $-1.5 \pm 1.2$ & $1.3 \pm 1.4$ & $1.6 \pm 2.0$ & $-12+2.2$ & $1.1 \pm 1.6$ & $-1.2 \pm 1.1$ & $1.1 \pm 1.6$ & $-1.4 \pm 1.5$ & $1.1 \pm 1.2$ & $10 \pm 1.7$ & $1.2 \pm 1.6$ & $-1.1 \pm 1.2$ & $1.4 \pm 1.3$ & $-1.3 \pm 1.5$ & $1.0 \pm 1.1$ \\
\hline & MTIF & $2.4 \pm 1.7$ & $598 \pm 1.5$ & $1.0 \pm 1.5$ & $1 . \pm 1.4$ & $1.6 \pm 1.7$ & $1.0 \pm 1.5$ & $3.3 \pm 1.3$ & $1.5 \pm 1.6$ & $1.5 \pm 1.7$ & $1.2 \pm 1.4$ & $1.1 \pm 1.3$ & $10 \pm 13$ & $-1.1 \pm 1.2$ & $-1,1 \pm 1.7$ & $-1.1 \pm 1.5$ & $1.2 \pm 1.4$ & $-1.2 \pm 1.5$ & $14 \pm 1.9$ & $-1.5 \pm 1.2$ & $1.0 \pm 1.3$ \\
\hline & compound & EtoH & $\mathrm{CdCl} 2$ & NIV & 4-NQO & cisplatin & FK506 & PCB 153 & 4-HNE & NaAs3+ & CXB & PhlP & 1-NPR & $\begin{array}{l}\text { 4,4'- } \\
\text { DDT }\end{array}$ & MDA & MTL & $\begin{array}{c}\text { 17B- } \\
\text { estradiol }\end{array}$ & OA & DMNA & 1Q & MBA \\
\hline
\end{tabular}

See footnote of Table 3 . 


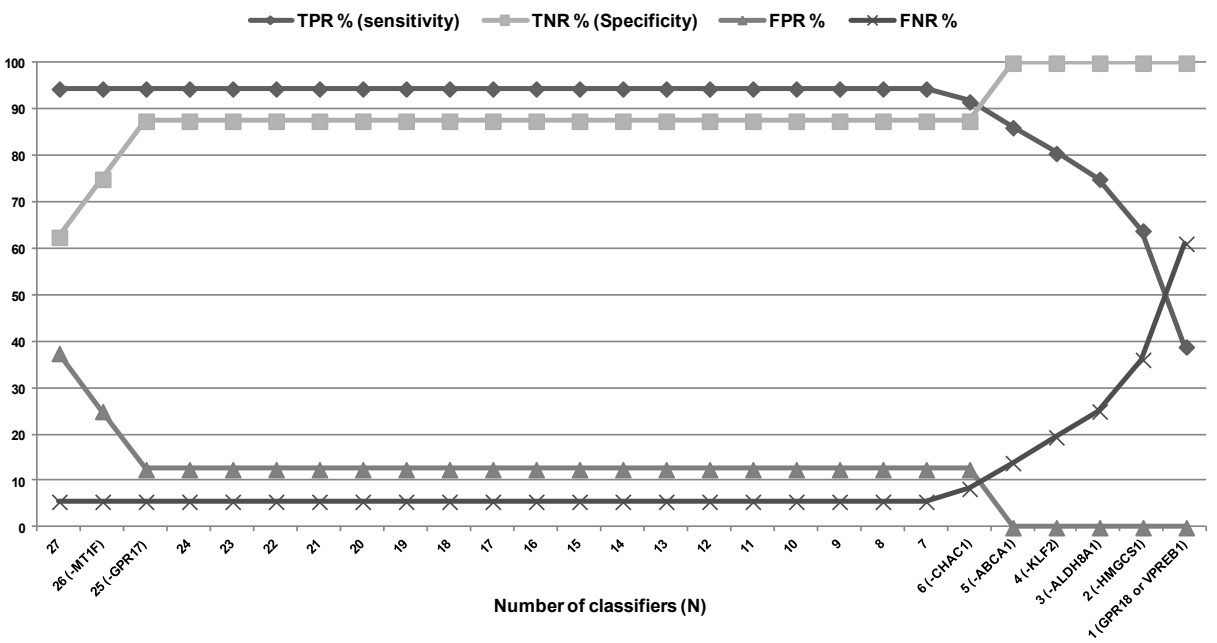

FIG. 2. Performance of different classifier gene set in the training set. The line figure above shows the discriminative power of different classifier sets in the training set. Seven is shown as the minimum number of genes to achieve the best performance. True positive rate (TPR\%) is calculated as $100 \times T P /(T P+F N)$. True negative rate (TNR\%) is calculated as $100 \times T N /(T N+F P)$. False positive rate is calculated as $100 \times F P /(F P+T N)$. False negative rate is calculated as $100 \times$ FN/ (FN+TP).

TABLE 5. Performance of different classifier gene sets in external verification dataset.

\begin{tabular}{c|c|c|c|c|c|c|c|c|c|c|c}
\hline $\begin{array}{c}\text { classifier } \\
\text { gene sets }\end{array}$ & $\begin{array}{c}\text { TP } \\
\text { (N) }\end{array}$ & $\begin{array}{c}\text { TN } \\
\text { (N) }\end{array}$ & $\begin{array}{c}\text { FP } \\
\text { (N) }\end{array}$ & $\begin{array}{c}\text { FN } \\
\text { (N) }\end{array}$ & $\begin{array}{c}\text { TPR } \% \\
\text { (sensitivity) }\end{array}$ & $\begin{array}{c}\text { TNR } \% \\
\text { (Specificity) }\end{array}$ & $\begin{array}{c}\text { FPR } \\
\%\end{array}$ & $\begin{array}{c}\text { FNR } \\
\%\end{array}$ & $\begin{array}{c}\text { FP } \\
\text { compounds }\end{array}$ & FN compounds & Accuracy \\
\hline $\mathrm{N}=25$ & 15 & 2 & 1 & 2 & 88 & 67 & 33 & 12 & IQ & $17 \mathrm{~B}$-estradiol, OA & 85 \\
$\mathrm{~N}=7$ & 13 & 2 & 1 & 4 & 76 & 67 & 33 & 24 & IQ & $\begin{array}{c}4-\mathrm{HNE}, \mathrm{NaAs} \mathrm{s}^{3+} \\
17 \mathrm{~B} \text {-estradiol, OA }\end{array}$ & 75 \\
\hline
\end{tabular}


TABLE 6. Functional themes based on the major $G O$ processes and molecular functions in which the 25 classifiers are involved.

\begin{tabular}{c|c}
\hline Category & Genes \\
\hline Regulation of cell cycle and apoptosis & CCNG2, CRIM1, CHAC1, HSPA1B, ID1, ID2 \\
Metabolism and transport of cholesterol & HMGCS1, ABCA1, ABCG1 \\
Cellular signal transduction & BZRAP1, ARRDC3, CRIM1, GPR18, KLHL24, CHAC1 \\
Cellular stress responses & KLF2, CHAC1, HSPA1B, HSPA5, NQO1, SRXN1, AK4, SLC7A11, \\
Immune regulation & KLF2, VPREB1, ID1, ID2, CEBPB \\
Protein metabolism & FBXO32, CRIM1, AK4 \\
Retinoic acid metabolism & ALDH8A1 \\
Regulation of transcription & ID1, ID2 \\
\hline
\end{tabular}

\section{Functional interpretation of the classifiers}

We categorized the 25 candidate classifier genes into eight functional themes, based on the major GO processes and molecular functions that these genes are involved in (Table 6). Then, using the ToxPi tool, we categorized the immunotoxic test compounds into separate functional sets by combining qRTPCR mRNA expression of these 25 genes with this functional information. The pie graphs for eight immunotoxicants with the highest overall ToxPi scores are shown in Fig. 3. These examples illustrate that these eight direct immunotoxicants can be categorized into four different functional groups, depicted in Fig. 3 a-d. The pie graphs also show that compounds with similar structures can affect the same functional themes. This is, for instance, the case for the parent compound ochratoxin A and S9treated ochratoxin A (Fig. 3a), and for the organotin compounds TBTC and TBTO (Fig. 3b). With respect to ochratoxin $A$, the S9-treated compound induced a greater effect on genes involved in protein metabolism and immune regulation than the parent compound. This observation indicates the bioactivation of ochratoxin A by the S9 treatment. Similar effects of S9 treatment were observed for two other compounds, being benzo[a]pyrene and cyclophosphamide (data not shown).

Compounds with different structures could also affect the same functional themes, such as $\mathrm{As}_{2} \mathrm{O}_{3}$, S9treated benzo[a]pyrene, and S9 treated cyclophosphamide (Fig. 3b). The insecticide lindane mainly affected protein metabolism and transcription, and forms another group itself (Fig. 3d). 


Regulation of cell and apoptosis $\quad$ Metabolism and transport of cholesterol
$\begin{aligned} & \text { Immune regulation } \\ & \text { Protein metabolism }\end{aligned}$ Retinoic acid metabolism

A. Retinoic acid metabolism, regulation of transcription, and cellular signal transduction

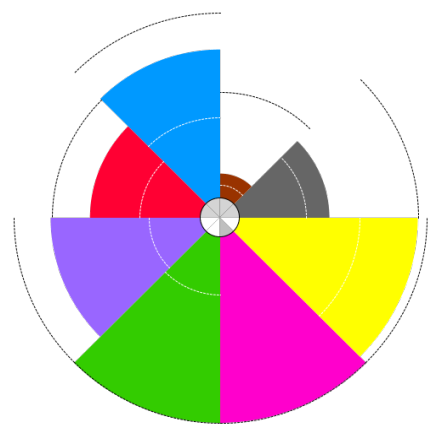

S9 treated ochratoxin A

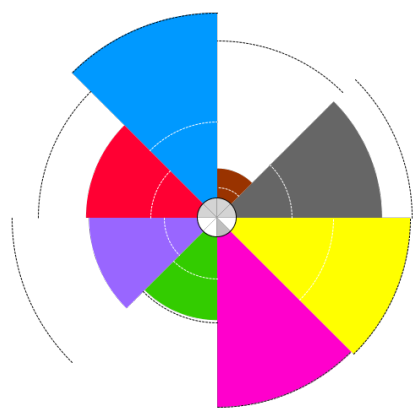

Ochratoxin A

B. Immune regulation and metabolism and transport of cholesterol

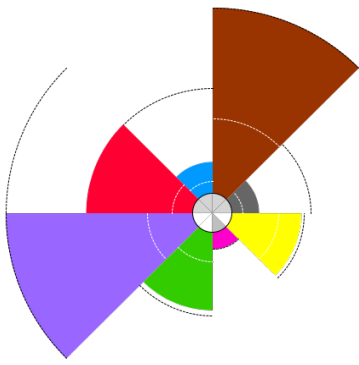

TBTC

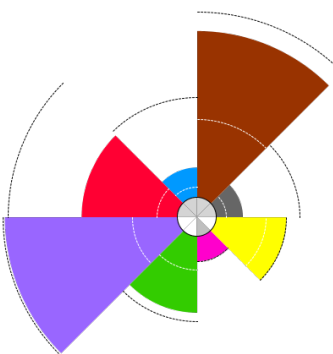

TBTO 
C. Cellular stress responses, regulation of transcription, and regulation of cell cycle and apoptosis

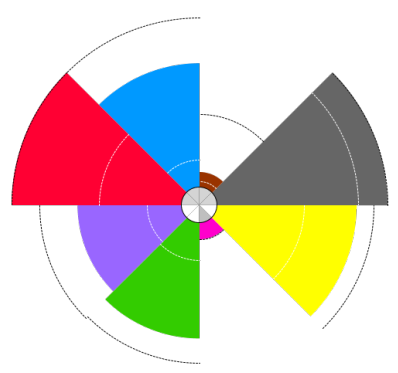

$\mathrm{As}_{2} \mathrm{O}_{3}$

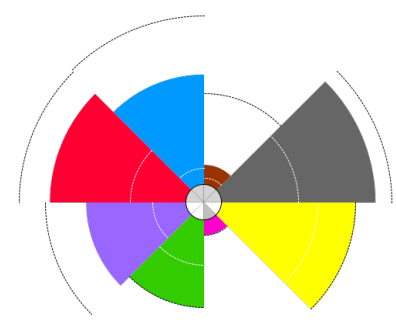

S9 treated benzo[a]pyrenen

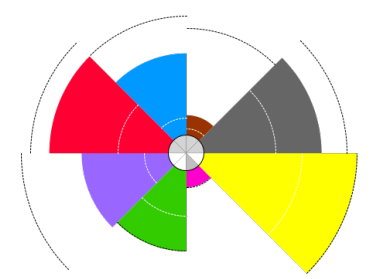

S9 treated cyclophosphamide

\section{Protein metabolism and regulation of transcription}

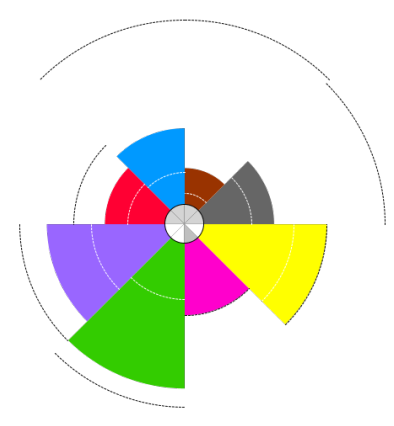

Lindane

FIG. 3. Visualization of the effects of example compounds on eight functional themes. The pie graphs visualize the effects of each compound on the functional themes as listed in Table 6. Each pie represents one compound. Each piece of the pie represents one single functional theme. The eight functional themes were weighted equally, so the graphic widths of all slices are equal. The size of each piece (the distance to the center, between 0 to 1) shows the ToxPi score that was calculated by the ToxPi software. Pie graphs are shown for the following compounds: a ochratoxin $\mathrm{A}( \pm S 9) ; \boldsymbol{b} \mathrm{As}_{2} \mathrm{O}_{3}$, benzo[a]pyrene (+S9) and cyclophosphamide (+S9); $\boldsymbol{c}$ TBTC and TBTO; $\boldsymbol{d}$ lindane. For the abbreviations of the compounds, see Table 1. 


\section{Discussion}

In the present study, we aimed at the identification and performance analysis of transcriptome-based functional classifiers that can be eventually used for the development of in vitro screening assays for direct immunotoxicity. An important starting point was to select immunotoxicants that cover a wide range of modes of action. A set of 25 classifier genes was identified based on the results of a training set of 36 immunotoxic and 8 nonimmunotoxic compounds. Moreover, this classifier set was validated by an external verification study that employed a set of 20 new compounds (17 immunotoxic and 3 non-immunotoxic). The accuracy of class prediction in the external verification set was $85 \%$.

Redundancy analysis revealed a smaller set of seven genes had the same discriminative power as the set of 25 genes in the training set (Fig. 2). Although a screening based on seven genes would be informative in distinguishing between immunotoxic and nonimmunotoxic compounds, the advantage of applying 25 genes would be that besides the class prediction, it also provides information on the modes of action of the compounds. Thus, part of these functional information would be lost if the screening would be based on only seven genes. For instance, three cellular processes that are potentially relevant for direct immunotoxicity were covered by the set of 25 genes, but not by the set of seven genes. These are growth inhibition and cell cycle arrest, represented by CCNG2 (Horne et al., 1997; Zimmermann et al., 2012), T cell lineage commitment (Wang et al., 2009; Jones-Mason et al., 2012) and regulation of transcription (Kim et al., 2003; Zheng et al., 2004), both being represented by ID1 and ID2. Moreover, the risk of false negatives would likely be higher for the set of seven genes because many compounds would be classified based on only one gene. This is also implied by the data of the external verification set, as the seven genes showed a higher false-negative rate than the 25 genes (Table 5). Therefore, with respect to a future in vitro screening assay based on the classifier sets that are described in this study, we would prefer to use the set of 25 genes.

The only false-positive compound in the external verification set is 2-amino-3-methyl-3H-imidazo[4,5F]quinoline (IQ). This compound was also used as a nonimmunotoxic control in a previous in vitro immunotoxicogenomics study by Hochstenbach et al., (2010). Hochstenbach et al. did not observe a clear separation of IQ from immunotoxic compounds either. This makes it questionable whether IQ is an appropriate negative control for immunotoxicity.

It is also worth pointing out that during the transcriptome profiling experiments (Shao et al., 2013), we observed transcriptional modulation of two histone deacetylases (HDACs) by certain immunotoxicants (i.e., HDAC4 by mycophenolic acid and HDAC8 by lindane, data not shown). Our result for mycophenolic acid is in line with the recent finding that this compound altered the gene expression of HDAC3 in bovine macrophages (Oh et al., 2013). HDACs are known to play crucial roles in epigenetic regulations (Delcuve et al., 2012). However, these genes were not selected for qRT-PCR verification based on our selection criteria. Therefore, it could be possible that the current classifier set is not sensitive enough to pick up compounds that can induce epigenetic changes. Further qRT-PCR verification experiments will be needed to verify whether these genes are good candidates to be included in the classifier gene set. 
For the development of an appropriate screening assay, the panel of test compounds needs to be extended in order to further determine the capability of the classifier gene set to identify immunotoxic compounds. The classifier genes described here might have less sensitivity to a novel class of immunotoxic chemicals or drugs, which has not been included in the training set of the present study. Therefore, additional classifier genes might need to be added to the current classifier gene set in order to enhance the sensitivity. Transferability between different laboratories will also be required as an important validation step. In our opinion, this issue can be relatively easy addressed since (1) Jurkat is a widely used and available cell line that can be cultured in standard medium; (2) the qRTPCR assays and the primers we used are commercially available; (3) the qRT-PCR experiments were already outsourced and were performed with certified SOPs.

Only a limited number of immunotoxicogenomics studies have been performed before. Most of these studies used a limited number of compounds and mainly focused on the modes of action of immunotoxicants (Ezendam et al., 2004; Baken et al., 2008; Katika et al., 2012; Katika et al., 2011). Hochstenbach et al., (2010) studied the transcriptomic changes in human PBMCs after exposure to 12 compounds. By applying recursive feature elimination based on a support vector machine (SVMRFE) algorithm, they identified a set of 48 genes as candidate classifier genes for direct immunotoxicity (Hochstenbach et al., 2010). All of the 12 compounds tested by Hochstenbach et al. were also examined in the present study. Four of these compounds were included in the training set, being aflatoxin $\mathrm{B} 1$, benzo[a]pyrene, DON, and TCDD. The other eight compounds were part of the external verification set. Most of the compounds were similarly considered as immunotoxicants or nonimmunotoxicants in both studies, except for 2-amino-1-methyl-6-phenylimidazo[4,5-b]pyridine (PhIP), which was used as a nonimmunotoxic model compound by Hochstenbach et al. 2010. In contrast, we allocated PhIP to the immunotoxic group, because this compound has been demonstrated to induce immunosuppression in a dose- dependent manner in mice (Davis et al., 1994). In spite of the overlap in test compounds, no overlap was found between the 48 genes of the Hochstenbach study and the 25 genes identified in the present study. The reason could be that for most of the compounds, the applied concentrations in the Hochstenbach study were much higher than the ones that were selected for the present study, and we did not choose these concentrations because they were cytotoxic in Jurkat cells. The other reason could be the difference in model system, as Hochstenbach et al. used human PBMCs, which are a mixture of primary cells (mainly $\mathrm{T}$ cells, B cells and NK cells), whereas we used a human T cell line. Although primary cells may be closer to the human situation, the application of a cell line is less laborious and more suitable for high throughput screening. In addition, the classifier gene set of the present study has the following advantages over the 48 gene set. It (1) has smaller chance of giving false negative results because it was trained on a much larger set of compounds (44 vs. 12 compounds); (2) has a better predictive performance because it has been validated by the compounds in the external verification set; (3) is a smaller gene set; and (4) used a shorter exposure time (6 vs. 24 h) and is therefore less timeconsuming. 
The screening assay to be developed on the basis of the identified classifier set can potentially be used for the safety evaluation of chemicals (REACH); preclinical safety evaluation of pharmaceuticals; and screening food, feed, soil, and water for the presence of immunotoxicants. Besides the application in the safety assessment of chemicals and drugs, this assay may also be used to search for new potential immunosuppressive drugs or functional food ingredients with immunosuppressive properties, which may contribute to the treatment of autoimmune diseases.

Although the performance of this assay was good in terms of accuracy and sensitivity, the specificity was less satisfactory. This might be caused by the fact that only three nonimmunotoxic compounds were tested in the external verification set. Therefore, in order to further determine the specificity of this classifier set, more nonimmunotoxic compounds should be tested. Since Jurkat is a T-cell line, it is conceivable that this assay is not able to identify immunotoxic compounds that specifically target immune cells other than T cells. In addition, in vitro bio-transformation of the compounds that are known to be bioactivated in vivo is needed, as several CYP enzymes are not expressed in Jurkat cell line (Nagai et al., 2002). Thus, Jurkat cells have a low metabolic capacity as compared to (primary) liver cells. Based on the above mentioned limitations, this Jurkat cell based screening assay should not be used as a separate assay, but as part of a battery of in vitro assays to screen compounds for potential immunotoxicity and prioritise compounds for further testing.

In conclusion, we have identified and validated a promising classifier gene set, whose expression profile after in vitro exposure of the Jurkat cell line resulted in a classification model that was $85 \%$ accurate in distinguishing immunotoxic from nonimmunotoxic compounds. Moreover, this set of 25 classifier genes can potentially be used for initial functional categorization of direct immunotoxicants. The in vitro assay that can be developed based on the results of the present study may complement animal free testing approaches that are currently being developed within the immunotoxicity field.

\section{Funding information}

This study was financially supported by the Netherlands Genomics Initiative, the Netherlands Toxicogenomics Centre (grant 05060510). 


\section{References}

Archibald, K., Coleman, R., and Foster, C. (2011). Open letter to UK Prime Minister David Cameron and Health Secretary Andrew Lansley on safety of medicines. The Lancet 377, 1915.

Baken, K. A., Pennings, J. L. A., Jonker, M. J., Schaap, M. M., de Vries, A., van Steeg, H., Breit, T. M., and van Loveren, H. (2008). Overlapping gene expression profiles of model compounds provide opportunities for immunotoxicity screening. Toxicol. Appl. Pharmacol. 226, 46-59.

Bouvier d'Yvoire, M., Bremer, S., Casati, S., Ceridono, M., Coecke, S., Corvi, R., Eskes, C., Gribaldo, L., Griesinger, C., Knaut, H., Linge, J. P., Roi, A., Zuang, V., Balls, M., Combes, R. D., and Bhogal, N. (2012). ECVAM and new technologies for toxicity testing new technologies for toxicity testing. Springer, New York, pp. 154-180.

Carlson, E. A., Li, Y., and Zelikoff, J. T. (2004). Benzo[a]pyrene-induced immunotoxicity in Japanese medaka (Oryzias latipes): relationship between lymphoid CYP1A activity and humoral immune suppression. Toxicol. Appl. Pharmacol. 201, 40-52.

Chan, M., Chan, M. W., Loh, T. W., Law, H. Y., Yoon, C. S., Than, S. S., Chua, J. M., Wong, C. Y., Yong, W. S., Yap, Y. S., Ho, G. H., Ang, P., and Lee, A. S. G. (2011). Evaluation of nanofluidics technology for high-throughput SNP genotyping in a clinical setting. J. Mol. Diagn. 13, 305-312.

Daaka, Y., Friedman, H., and Klein, T. W. (1996). Cannabinoid receptor proteins are increased in Jurkat, human T-cell line after mitogen activation. J. Pharmacol. Exp. Ther. 276, 776-783.

Davis, D. A., Archuleta, M. M., Born, J. L., Knize, M. G., Felton, J. S., and Burchiel, S. W. (1994). Inhibition of humoral immunity and mitogen responsiveness of lymphoid cells following oral administration of the heterocyclic food mutagen 2-amino-1-methyl-6-phenylimidazo[4,5-b]pyridine (PhIP) to B6C3F1 mice. Fundam. Appl. Toxicol 23, 81-86.

De Jong, W. H., and van Loveren, H. (2007). Screening of xenobiotics for direct immunotoxicity in an animal study. Methods 41, 3-8.

Delcuve, G., Khan, D., and Davie, J. (2012). Roles of histone deacetylases in epigenetic regulation: emerging paradigms from studies with inhibitors. Clin Epigenet 4, 5.

Ekhart, C., Rodenhuis, S., Beijnen, J., and Huitema, A. (2009). Carbamazepine induces bioactivation of cyclophosphamide and thiotepa. Cancer Chemother. Pharmacol. 63, 543-547.

EMA (2000). Note for Guidance on Repeated Dose Toxicity, CPMP/SWP/1042/99.

EPA (1998). Health Effects Test Guidelines, Immunotoxicity, OPPTS 870. 7800.

Ezendam, J., Staedtler, F., Pennings, J., Vandebriel, R. J., Pieters, R., Boffetta, P., Harleman, J. H., and Vos, J. G. (2004). Toxicogenomics of subchronic hexachlorobenzene exposure in Brown Norway rats. Environ. Health. Perspect. 112, 782-791.

FDA (2002). Guidance for industry: immunotoxicology evaluation of investigational new drugs.

Fernández-Riejos, P., Goberna, R., and Sánchez-Margalet, V. (2008). Leptin promotes cell survival and activates Jurkat $\mathrm{T}$ lymphocytes by stimulation of mitogen-activated protein kinase. Clin. Exp. Immunol. 151, 505-518. 
Fischer, A. M., Mercer, J. C., Iyer, A., Ragin, M. J., and August, A. (2004). Regulation of CXC chemokine receptor 4-mediated migration by the Tec family tyrosine kinase ITK. J. Biol. Chem. 279, 29816-29820.

Hochstenbach, K., van Leeuwen, D. M., Gmuender, H., Stølevik, S. B., Nygaard, U. C., Løvik, M., Granum, B., Namork, E., van Delft, J. H., and van Loveren, H. (2010). Transcriptomic profile indicative of immunotoxic exposure: in vitro studies in peripheral blood mononuclear cells. Toxicol. Sci. 118, 1930.

Hollants, S., Redeker, E. J., and Matthijs, G. (2012). Microfluidic amplification as a tool for massive parallel sequencing of the familial hypercholesterolemia genes. Clin. Chem. 58, 717-724.

Horne, M. C., Donaldson, K. L., Goolsby, G. L., Tran, D., Mulheisen, M., Hell, J. W., and Wahl, A. F. (1997). Cyclin G2 Is up-regulated during growth inhibition and B cell antigen receptor-mediated cell cycle arrest. J. Biol. Chem. 272, 12650-12661.

Institóris, L., Siroki, O., Dési, I., Lesznyák, J., Serényi, P., Szekeres, É., and Petri, I. (1998). Extension of the protocol of OECD guideline 407 (28-day repeated dose oral toxicity test in the rat) to detect potential immunotoxicity of chemicals. Hum. Exp. Toxicol. 17, 206-211.

Jang, J., Simon, V., Feddersen, R., Rakhshan, F., Schultz, D., Zschunke, M., Lingle, W., Kolbert, C., and Jen, J. (2011). Quantitative miRNA expression analysis using fluidigm microfluidics dynamic arrays. BMC Genomics 12, 144.

Jones-Mason, Mary E., Zhao, X., Kappes, D., Lasorella, A., lavarone, A., and Zhuang, Y. (2012). E protein transcription factors are required for the development of CD4+ lineage T cells. Immunity $\mathbf{3 6}$, 348-361.

Katika, M. R., Hendriksen, P. J., Shao, J., van Loveren, H., and Peijnenburg, A. (2012). Transcriptome analysis of the human $\mathrm{T}$ Iymphocyte cell line Jurkat and human peripheral blood mononuclear cells exposed to deoxynivalenol (DON): New mechanistic insights. Toxicol. Appl. Pharmacol. 264, 51-64.

Katika, M. R., Hendriksen, P. J., van Loveren, H., and Peijnenburg, A. (2011). Exposure of Jurkat cells to bis (tri-n-butyltin) oxide (TBTO) induces transcriptomics changes indicative for ER- and oxidative stress, T cell activation and apoptosis. Toxicol. Appl. Pharmacol. 254, 311-322.

Kawabata, T. T., and Evans, E. W. (2012). Development of immunotoxicity testing strategies for immunomodulatory drugs. Toxicol. Pathol. 40, 288-293.

Kim, H. J., Kim, J. Y., Park, Y. Y., and Choi, H. S. (2003). Synergistic activation of the human orphan nuclear receptor SHP gene promoter by basic helix-loop-helix protein E2A and orphan nuclear receptor SF-1. Nucleic Acids Res. 31, 6860-6872.

Lankveld, D. P., Loveren, H., Baken, K. A., Vandebriel, R. J., and Dietert, R. R. (2010). In vitro testing for direct immunotoxicity: state of the art immunotoxicity testing. In: Immunotoxicity testing (J. M. Walker, Ed.), pp. 401-423. Humana Press, New York.

Luebke, R. W., Holsapple, M. P., Ladics, G. S., Luster, M. I., Selgrade, M., Smialowicz, R. J., Woolhiser, M. R., and Germolec, D. R. (2006). Immunotoxicogenomics: the potential of genomics technology in the immunotoxicity risk assessment process. Toxicol. Sci. 94, 22-27. 
Makar, R. S., Lipsky, P. E., and Cuthbert, J. A. (1994). Non-sterol regulation of low density lipoprotein receptor gene expression in T cells. J. Lipid Res. 35, 1888-1895.

Manderville, R. A. (2005). A case for the genotoxicity of ochratoxin A by bioactivation and covalent DNA adduction. Chem. Res. Toxicol. 18, 1091-1097.

Morrison, L. (2010). Basic principles of fluorescence and energy transfer applied to Real-Time PCR. Mol. Biotechnol. 44, 168-176.

Nagai, F., Hiyoshi, Y., Sugimachi, K., and Tamura, H. O. (2002). Cytochrome P450 (CYP) expression in human myeloblastic and lymphoid cell lines. Biol. Pharm. Bull 25, 383-385.

Oh, S. Y., Balch, C., Cliff, R., Sharma, B., Boermans, H., Swamy, H. V., Quinton, V. M., and Karrow, N. (2013). Exposure to penicillium mycotoxins alters gene expression of enzymes involved in the epigenetic regulation of bovine macrophages (BoMacs). Mycotoxin Res. 29, 1-9.

Perel, P., Robert, I., E, S., Wheble, P., Briscoe, C., Sandercock, P., Macleod, M., Mignini, L., Jayaram, P., and Khan, K. (2007). Comparison of treatment effects between animal experiments and clinical trials: systematic review. BMJ 334, 197-200.

Ramsdell, H., Parkinson, A., Eddy, A., and Eaton, D. (1991). Bioactivation of aflatoxin B1 by human liver microsomes: role of cytochrome P450 IIIA enzymes. Toxicol. Appl. Pharmacol. 108, 436-47.

Reif, D. M., Sypa, M., Lock, E. F., Wright, F. A., Wilson, A., Cathey, T., Judson, R. R., and Rusyn, I. (2013). ToxPi GUI: an interactive visualization tool for transparent integration of data from diverse sources of evidence. Bioinformatics 29, 402-403.

Shao, J., Katika, M. R., Schmeits, P. C. J., Hendriksen, P. J., van Loveren, H., Peijnenburg, A. A., and Volger, O. L. (2013). Toxicogenomics-based identification of mechanisms for direct immunotoxicity. Toxicol. Sci. 135, 328-346

Tugwood, J. D., Hollins, L. E., and Cockerill, M. J. (2003). Genomics and the search for novel biomarkers in toxicology. Biomarkers 8, 79-92.

Wang, H. C., Perry, S. S., and Sun, X. H. (2009). Id1 attenuates notch signaling and impairs T-cell commitment by elevating Deltex1 expression. Mol. Cell. Biol. 29, 4640-4652.

Waters, M. D., and Fostel, J. M. (2004). Toxicogenomics and systems toxicology: aims and prospects. Nat. Rev. Genet. 5, 936-948.

Zheng, W., Wang, H., Xue, L., Zhang, Z., and Tong, T. (2004). Regulation of cellular senescence and p16INK4a expression by Id1 and E47 proteins in human diploid fibroblast. J. Biol. Chem. 279, 3152431532.

Zimmermann, M., Arachchige-Don, A. S., Donaldson, M. S., Dallapiazza, R. F., Cowan, C. E., and Horne, M. C. (2012). Elevated cyclin G2 expression intersects with DNA damage checkpoint signaling and is required for a potent G2/M checkpoint arrest response to Doxorubicin. J. Biol. Chem. 287, 22838-22853. 


\title{
CHAPTER 7
}

\section{Successful validation of genomic biomarkers for human immunotoxicity in Jurkat $\mathrm{T}$ cells in vitro}

\author{
Peter C. J. Schmeits, Jia Shao, Danique A. van der Krieken, Oscar L. Volger, \\ Henk van Loveren, Ad A. C. M. Peijnenburg, and Peter J. M. Hendriksen
}

J. Appl. Toxicol. in press.

Supplementary materials can be found at: http://onlinelibrary.wiley.com/doi/10.1002/jat.3079/suppinfo 


\begin{abstract}
Previously, we identified 25 classifier genes that were able to assess immunotoxicity using human Jurkat $T$ cells. The present study aimed to validate these classifiers. For that purpose, Jurkat cells were exposed for $6 \mathrm{~h}$ to subcytotoxic doses of nine immunotoxicants, five nonimmunotoxicants, and four compounds for which human immunotoxicity has not yet been fully established. RNA was isolated and subjected to Fluidigm qRT-PCR analysis. The sensitivity, specificity and accuracy of the screening assay as based on the nine immunotoxicants and five nonimmunotoxicants used in this study were $100 \%, 80 \%$ and $93 \%$, respectively, which is better than the performance in our previous study. Only one compound was classified as false positive (benzo-e-pyrene). Of the four potential (non-)immunotoxicants, chlorantraniliprole and Hidrasec were classified immunotoxic and sunset yellow and imidacloprid as nonimmunotoxic. ToxPi analysis of the PCR data provided insight in the molecular pathways that were affected by the compounds. The immunotoxicants 2,3-dichloropropanol and cypermethrin, although structurally different, affected protein metabolism and cholesterol biosynthesis and transport. In addition, four compounds, i.e. chlorpyrifos, aldicarb, benzoe-pyrene and anti-CD3, affected genes in cholesterol metabolism and transport, protein metabolism and transcription regulation. QRT-PCR on eight additional genes coding for similar processes as defined in ToxPi analyses, supported these results. In conclusion, the 25 immunotoxic classifiers performed very well in a screening with new nonimmunotoxic and immunotoxic compounds. Therefore, the Jurkat screening assay has great promise to be applied within a tiered approach for animal free testing of human immunotoxicity.
\end{abstract}




\section{Introduction}

Direct immunotoxicity is defined as the action of a compound on components of the immune system that lead to either immunostimulation or immunosuppression. The assessment of direct immunotoxicity is an important part of the overall toxicological investigation of chemicals (Lankveld, et al. 2010). Several authorities including the European Medicines Agency (EMEA 2006) and the US Food and Drug Administration (FDA 2002) emphasize the need to evaluate immunotoxic effects during the preclinical phase of drug development. Other authorities such as the Organisation for Economic Cooperation and Development (OECD) (Institoris, et al. 1998) and the Environmental Protection Agency (EPA 2013) stress the need for safety assessment of food contaminants and environmental pollutants. Current immunotoxicity screening still focuses on rodent in vivo models. Since the implementation of REACH, that requires the evaluation of chemical hazards for chemicals of which the yearly production exceeds one tonne, progress has been made towards development of in vitro alternatives for immunotoxicity testing (EC 2006).

In our previous work, using the in vitro Jurkat T cell model, we identified and validated 25 biomarker genes representative for different mechanisms underlying direct immunotoxicity (Shao, et al. 2014, Shao, et al. 2013) (Table 1). Jurkat T cells were chosen since they are easy to work with, cheap, readily available and of human origin, making extrapolation to human hazard easier than an animal cell model. Jurkat cells have also been applied in assessing the effect of compounds on mitogeninduced proliferation and migration (Fernandez-Riejos, et al. 2008, Fischer, et al. 2004).

On the basis of the outcome of the previous study, two important questions were raised. Firstly, how does the screening system perform towards new classes of immunotoxicants that have not been tested yet? This question is relevant because the biomarkers are based on a set of chemicals belonging to a variety of compound classes such as metals, mycotoxins, herbicides, insecticides, fungicides. Therefore, how this biomarker based system will perform when exposing to a compound from a class that has not been tested should be further investigated. Secondly, only a limited number of nonimmunotoxic compounds has been tested, which resulted in a less satisfactory specificity. How will this screening system perform when more nonimmunotoxicants are included? To answer these important issues, new compounds were searched for in literature and reports.

In total we selected nine known immunotoxicants, five known nonimmunotoxicants and four compounds for which it was not fully established whether they are immunotoxic or not (Table 2). Known immunotoxicants from new compound classes include the chloropropanol food contaminant 2,3-dichloro-1-propanol (2,3-DCP) (Lu, et al. 2013), the biological anti-CD3 (Ishiguro and Xavier 2004, Weetall, et al. 2002), the organophosphorus pesticide chlorpyrifos which causes immunotoxicity in T cells and NK cells (Dunier, et al. 1991, Noworyta-Glowacka, et al. 2012), the lectin phytohaemagglutinin (PHA-L) (Mitsuishi, et al. 1986) and the poly-brominated flame retardant tetrabromobisphenol A (TBBPA) (Birnbaum and Staskal 2004, Pullen, et al. 2003). In addition, immunotoxic compounds from classes tested before were included: the insecticides cypermethrin and 
aldicarb (Jin, et al. 2011), and the mycotoxin zearalenone (Abbes, et al. 2013).The insecticide aldicarb increased T cell populations in women drinking contaminated water (Mirkin, et al. 1990), however aldicarb was assessed to be nonimmunotoxic in mice (Thomas, et al. 1987). Arsenic trioxide was already tested before in the Jurkat screening model and is tested again as a positive control as it showed the largest effects on the biomarker genes (Shao, et al. 2013).

Since the results of compounds that have been tested as nonimmunotoxic are less likely to be published, a publication bias exists for nonimmunotoxic compounds. Therefore, searches for non immunotoxicants also concerned governmental reports like those from the European Chemicals Agency (ECHA) and the Australian Pesticides and the Veterinary Medicines Authority (APVMA). Known nonimmunotoxicants selected in this study were ametoctradin (APVMA), arsenobetaine (Borak and Hosgood 2007, Sakurai, et al. 2004), carbon tetrachloride (CCL4) (Smialowicz, et al. 1991), diuron (ECHA 2014) and benzo-e-pyrene (White, et al. 2012).

A third category contains compounds of which immunotoxicity has not been definitively been observed. Four compounds were included being sunset yellow FCF, racecadotril (Hidrasec), chlorantraniliprole (trade name Rynaxypyr) and imidacloprid. Sunset yellow FCF is a food contaminant that was tested immunotoxic in mice (Hashem, et al. 2010, Yadav, et al. 2013). Sunset yellow FCF reduced the number of monocytes to $50 \%$ in mice in vivo without affecting other immune cells (Hashem, et al. 2010). Hidrasec, which is used as treatment for diarrhoea, was assessed to be nonimmotoxic in rodents (non-peer reviewed publications). Though, the information leaflet mentions that up to $1 \%$ of the users suffered from rash and erythema, which are clinical signs of hypersensitivity of the human skin to chemicals. Also chlorantraniliprole was evaluated as nonimmunotoxic in rodents (non-peer reviewed publications). However, chlorantraniliprole is suspected to act on release of calcium from intracellular calcium stores (Lahm, et al. 2005) which is an important process in T cell activation. Therefore it was decided to include this drug in the present study as well. Imidacloprid is a neonicotinoid insecticide and is tested immunotoxic in rats and mice (Badgujar, et al. 2013, Gawade, et al. 2013). Nevertheless, there is little known about the effects of this compound on the human immune system and on human-derived immune cells in vitro.

The aim of this study is twofold. The first aim is to validate the 25 biomarkers previously identified by screening new immunotoxic and nonimmunotoxic compounds. In our previous study the sensitivity, specificity and accuracy were 88,67 and $85 \%$ (Shao, et al. 2014). The low specificity was likely due to the fact that only three non immunotoxic controls were examined. Therefore, in the present work, the performance of the classifier genes is tested using an extended set of nonimmunotoxic controls. The second aim is to predict the immunotoxicity of five compounds for which insufficient data is available. Next to the 25 marker genes identified before, we selected eight additional genes that are known to be biomarkers for specific processes including $T$ cell activation, ER stress, cholesterol homeostasis and cell cycle (Table 4). It was envisaged that the response of these genes in combination with the 25 markers for immunotoxicity will provide insight in the mechanisms of action of the compounds. For assessing the mRNA expression response of the 33 genes to the 19 compounds 
in Jurkat cells, we used a Fluidigm high-throughput PCR system, which has proven to be a fast and reliable system to study gene expression (Shao, et al. 2014). The set of 25 biomarkers yielded a sensitivity of $100 \%$, specificity of $80 \%$ and accuracy of $93 \%$. This performance was better than that obtained in the previous validation experiment (Shao, et al. 2014).

\section{Materials and Methods}

Chemicals. All chemicals were ordered from Sigma-Aldrich (Zwijndrecht, the Netherlands), except anti-CD3 and IgG isotype control (Abcam, Cambridge, UK).

Cell culture. The human T-lymphocyte cell line (Jurkat) was obtained from the American Type Culture Collection (ATCC). Jurkat cells were cultured in RPMI-1640 medium supplemented with 10\% Fetal Calf Serum, $2 \mathrm{mM}$ glutamine, $1 \mathrm{mM}$ sodium pyruvate, $1 \mathrm{mM}$ nonessential amino acids, $100 \mathrm{U} / \mathrm{ml}$ penicillin, and $100 \mu \mathrm{g} / \mathrm{ml}$ streptomycin (Invitrogen Life Science, Breda, The Netherlands). Cells were cultured at $37^{\circ} \mathrm{C}$ with $5 \% \mathrm{CO}_{2}$ in a humidified atmosphere. The medium was refreshed three times a week.

ATPlite assay. Cell viability was tested using the ATPlite assay (Perkin Elmer, Oosterhout, The Netherlands) which is based on the production of light caused by the reaction of ATP with luciferase and D-luciferin. The emitted light is proportional to the amount of ATP, which is a marker for cell viability. Jurkat cells were plated $20 \mathrm{~h}$ before exposure in a 96 well plate $(220,000$ cells/well). Exposure was done in triplicate in $100 \mu \mathrm{l}$ medium in 96 well plates for $24 \mathrm{~h}$ to increasing concentrations of compounds, or to the vehicle controls. After exposure, the assay was performed according the manufacturer's protocol.

Exposures. Jurkat cells (passage number between 15 -19) were seeded in 6-well plates containing $2.7 \mathrm{ml}$ (750,000 cells/well) per well. After growing the cells for $20 \mathrm{~h}$, exposure was initiated by adding $0.3 \mathrm{ml}$ medium containing non-cytotoxic concentration of the compounds or vehicle controls. Subsequently, cells were exposed to the compounds for $6 \mathrm{~h}$. The maximum final DMSO concentration in the medium was $0.1 \%(\mathrm{v} / \mathrm{v})$ for all the samples which had no effect on viability. For each compound, exposures were performed on three different days. IgG was used as a control for the anti-CD3 exposures. DMSO was not present in either anti-CD3 or IgG treatments.

RNA isolation and quality control. After exposure, the culture medium was removed after centrifugation of the cell suspension $\left(5 \mathrm{~min}\right.$ at $\left.300 \mathrm{~g}, 4^{\circ} \mathrm{C}\right)$. The resulting cell pellet was homogenized 
in $600 \mu \mathrm{l}$ lysis buffer (RLT) (Qiagen, Venlo, The Netherlands) supplemented with $10 \% \beta$ mercaptoethanol and stored at $-80^{\circ} \mathrm{C}$ until further processing. RNA was isolated with the QIAshredder kit (Qiagen) according to the manufacturer's protocol. Subsequently, RNA was purified using the mRNeasy kit (Qiagen) including DNase treatment according to the manufacturer's protocol. RNA yield was assessed spectrophotometrically (NanoDrop 2000, Isogen Life Science, De Meern, The Netherlands).

Quantitative Real-Time PCR verification. Detection of biomarker gene expression changes was performed at ServiceXS (ServiceXS BV, Leiden, The Netherlands) using the 96.96 BioMark Dynamic Array for Real-Time PCR (Fluidigm Corporation, San Francisco, CA), according to the manufacturer's instructions. cDNA samples were synthesized using miScript Reverse Transcription kit according to the manufacturer's protocol (Qiagen). Before use on the BioMark array, the cDNA was first subjected to 14 cycles of specific target amplification (STA) using a $0.2 x$ mixture of all Taqman Gene Expression assays in combination with the Taqman PreAmp Master Mix (Applied Biosystems), followed by 5 -fold dilution. Water was included as no template control (NTC) at the indicated positions. The no template controls were also included in the STA reaction, to serve as a true negative control for the entire procedure. After the $5 x$ dilution, thermal cycling and real-time imaging of the BioMark array was done on the BioMark instrument, and threshold cycle $(\mathrm{Ct})$ values were extracted using the BioMark RealTime PCR analysis software. Ct values were used to determine compound effects on mRNA expression levels. Pair-wise combinations of all samples were made with each of the assays in duplicate on the array. The empty assay positions were filled with no assay controls, in which the $20 \mathrm{x}$ assay mix was substituted with water). The default Taqman PCR protocol was used on the BioMark instrument with an annealing temperature of $60^{\circ} \mathrm{C}$ and a total of 35 cycles of PCR.

Analyses of qRT-PCR data. At the end of each PCR cycle, data was collected from the 9,216 reaction chambers on each array, and Ct values were extracted using the BioMark Real-Time PCR analysis software version 3.0.2. The quality threshold was set at 0.65 (default value). The relative mRNA expression levels were calculated for each individual sample by applying the delta-delta-CT method. Three genes were selected as reference genes, based on the low variability of the relative mRNA expression levels quantified by the microarrays performed before (Shao, et al. 2013), being beta-2macroglobulin (B2M, highly abundant), Golgi to ER traffic protein 4 (GET4, lowly abundant), and phosphatidylinositol glycan anchor biosynthesis class G1 (PIGG). Compounds are considered immunotoxic when one or more of the classifier genes are affected by factor $\geq 2$ vs. the control in at least two out of three replicates. Clustering of genes was performed using Cluster v3.0 (uncentered correlation; average linkage clustering) developed by Stanford University. Treeview was used for visualisation of the clustering analysis. 
Functional interpretation based on ToxPi analysis. The 25 classifiers were divided into eight functional classes (Table 3 ). The effects of each compound on these functional themes were visualized using ToxPi software from the US EPA (Reif, et al. 2013). The mRNA levels of the 25 classifier genes of the 16 compounds were used as the input for ToxPi software. ToxPi calculates a ToxPi score for each compound on each functional theme, and ranks the compounds by their overall ToxPi scores. The ToxPi score (between 0 to 1 ) is calculated by dividing the results of each chemical on the genes that are involved in this functional theme by the maximum results on the same genes from the 16 compounds.

$q R T-P C R$ on additional genes for functional confirmation. Eight genes were selected for additional qRT-PCR experiments to confirm the functional themes that were used in ToxPi analyses. These genes were involved in processes such as T cell activation, apoptosis, ER stress, cholesterol homeostasis, immune regulation, cell cycle and regulation of transcription (Table 4). QRT-PCR procedure was identical to the methods described above.

Hierarchical clustering. Hierarchical clustering was performed with the programs Cluster (uncentered correlation; average linkage clustering) and Treeview (Eisen, et al. 1998). Red and green indicate upand downregulation vs. average expression of control samples. 
TABLE 1. Overview of 28 primers that were used as classifiers of immunotoxicity. The latter three primers (italics) were used as reference genes.

\begin{tabular}{|c|c|c|c|}
\hline Gene Symbol & Description & mRNA accession & Taqman assay \\
\hline ABCA1 & ATP-binding cassette, sub-family A ( $A B C 1)$, member 1 & NM_005502 & Hs01059118_m1 \\
\hline ABCG1 & ATP-binding cassette, sub-family G (WHITE), member 1 & NM_004915 & Hs00245154_m1 \\
\hline AK4 & adenylate kinase 4 & NM_001005353 & Hs03405743_g1 \\
\hline ALDH8A1 & aldehyde dehydrogenase 8 family, member $\mathrm{A} 1$ & NM_001193480 & Hs00988965_m1 \\
\hline ARRDC3 & arrestin domain containing 3 & NM_020801 & Hs00385845_m1 \\
\hline BZRAP1 & $\begin{array}{l}\text { benzodiazapine receptor (peripheral) associated protein } \\
1\end{array}$ & NM_004758 & Hs00270490_m1 \\
\hline CCNG2 & cyclin G2 & NM_004354 & Hs00171119_m1 \\
\hline CEBPB & CCAAT/enhancer binding protein (C/EBP), beta & NM_005194 & Hs00270923_s1 \\
\hline $\mathrm{CHAC} 1$ & ChaC, cation transport regulator homolog 1 (E. coli) & NM_001142776 & Hs00225520_m1 \\
\hline CRIM1 & $\begin{array}{l}\text { cysteine rich transmembrane BMP regulator } 1 \text { (chordin- } \\
\qquad \text { like) }\end{array}$ & NM_016441 & Hs00212750_m1 \\
\hline FBXO32 & F-box protein 32 & NM_001242463 & Hs01041408_m1 \\
\hline GPR18 & G protein-coupled receptor 18 & NM_001098200 & Hs00245542_m1 \\
\hline HMGCS1 & 3-hydroxy-3-methylglutaryl-CoA synthase 1 (soluble) & NM_001098272 & Hs00940429_m1 \\
\hline HSPA1B & heat shock $70 \mathrm{kDa}$ protein $1 \mathrm{~B}$ & NM_005346 & $\mathrm{Hs} 01040501-\mathrm{sH}$ \\
\hline HSPA5 & $\begin{array}{l}\text { heat shock } 70 \mathrm{kDa} \text { protein } 5 \text { (glucose-regulated protein, } \\
\qquad 78 \mathrm{kDa})\end{array}$ & NM_005347 & Hs00946084_g1 \\
\hline ID1 & $\begin{array}{l}\text { inhibitor of DNA binding } 1 \text {, dominant negative helix-loop- } \\
\text { helix protein }\end{array}$ & NM_002165 & Hs00357821_g1 \\
\hline
\end{tabular}




\begin{tabular}{|c|c|c|c|}
\hline ID2 & $\begin{array}{l}\text { inhibitor of DNA binding 2, dominant negative helix-loop- } \\
\qquad \text { helix protein }\end{array}$ & NM_002166 & Hs00747379_m1 \\
\hline KLF2 & Kruppel-like factor 2 (lung) & NM_016270 & Hs00360439_g1 \\
\hline KLHL24 & kelch-like 24 (Drosophila) & NM_017644 & Hs00214210_m1 \\
\hline NQO1 & $\mathrm{NAD}(\mathrm{P}) \mathrm{H}$ dehydrogenase, quinone 1 & NM_000903 & Hs01045995_m1 \\
\hline SLC7A11 & $\begin{array}{l}\text { solute carrier family } 7 \text { (anionic amino acid transporter } \\
\text { light chain, xc- system), member } 11\end{array}$ & NM_014331 & Hs00921938_m1 \\
\hline SRXN1 & sulfiredoxin 1 & NM_080725 & Hs00607800_m1 \\
\hline TM6SF1 & transmembrane 6 superfamily member 1 & NM_001144903 & Hs00224823_m1 \\
\hline VLDLR & very low density lipoprotein receptor & NM_001018056 & Hs01047538_m1 \\
\hline VPREB1 & pre-B lymphocyte 1 & NM_007128 & Hs00356766_g1 \\
\hline$B 2 M$ & $\beta 2$ microglobulin, present on all nucleated cells & NM_004048 & H2000984230_m1 \\
\hline GET4 & Golgi To ER Traffic Protein 4 Homolog & NM_015949.2 & Hs00944514_m1 \\
\hline PIGG & $\begin{array}{l}\text { Phosphatidylinositol Glycan Anchor Biosynthesis, Class } \\
\text { G }\end{array}$ & NM_017733.3 & Hs01107608_m1 \\
\hline
\end{tabular}




\section{Results}

ATPlite assay

The viability of Jurkat cells that were exposed to the different compounds was assessed by means of the ATPlite assay. Compound concentrations that lead to a decrease in viability of $20 \%$ or less after $24 \mathrm{~h}$ incubation (CV80) were selected for gene expression analysis, as published before (Schmeits, et al. 2013). For some compounds, the viability did not decrease after exposure to the highest concentration not giving rise to precipitation. In that case, this highest concentration was taken for further analysis. An overview of the viability data is presented in Figure 1. The concentrations that were selected for exposures are listed in Table 2.

\section{Biomarker characteristics}

The qRT-PCR data were subjected to hierarchical clustering analysis (the outcome is visualized in the heat-map in Figure 2). The results of the qRT-PCR analysis are also shown in Supplementary Figure 1. All nine known immunotoxicant are classified as immunotoxic based on the 25 gene markers. The sensitivity is therefore $100 \%$. For the known nonimmunotoxicants, four out of five were correctly classified as nonimmunotoxic. Benzo-e-pyrene downregulated one gene (HMGCS1) and is consequently classified as false-positive. The specificity of this set is therefore $80 \%$. The overall accuracy is $93 \%$. The performance characteristics of the present analysis are also shown in supplementary table 1.

\section{Compounds with unknown immunotoxic properties}

This study included four compounds for which the immunotoxic properties have not been precisely described. The reason for including these compounds was to verify whether the expression profiles of these 25 marker genes can provide more insight into the modes of action and point towards possible immunotoxic properties of these compounds. Chlorantraniliprole upregulates two genes (GPR18 and SLC7A11) and is classified as immunotoxic. Hidrasec upregulates seven genes and downregulates one and is also classified as immunotoxic. Imidacloprid and Sunset yellow do not affect any of the genes and are therefore considered not immunotoxic 
TABLE 2. Overview of the compounds, classes and concentrations used. Compounds ( $n=18$ ) are divided in immunotoxic ( $n=9$, top), nonimmunotoxic ( $n=5$, middle) and unknowns ( $n=4$, bottom).

\begin{tabular}{|c|c|c|c|}
\hline Compound name & Class & $\begin{array}{l}\text { Concentration } \\
(\mu \mathrm{M}) \mathrm{CV} 80\end{array}$ & Immunotoxicity \\
\hline 2,3-dichloro-1-propanol & Food contaminant, chloropropanol & 6000 & Yes \\
\hline Aldicarb & Insecticide & 1000 & Yes \\
\hline Anti-CD3 & Biological & $2.5 \mathrm{ng} / \mu \mathrm{l}$ & Yes \\
\hline Arsenic trioxide & Metal & 3 & Yes \\
\hline Chlorpyrifos & Organophosphorous pesticide & 1000 & Yes \\
\hline Cypermethrin & Insecticide & 1000 & Yes \\
\hline Phytohaemmagluttinin & Lectin & $100 \mathrm{ng} / \mathrm{ml}$ & Yes \\
\hline Tetrabromobisphenol A & Flame retardant & 75 & Yes \\
\hline Zearalenone & Fungal mycotoxin & 50 & Yes \\
\hline Ametoctradin & Fungicide & 25 & No \\
\hline Arsenobetaine & Organoarsenic compound & 20 & No \\
\hline Benzo[e]pyrene & Polyaromatic hydrocarbon & 250 & No \\
\hline Carbon tetrachloride & Organic compound & 1000 & No \\
\hline Diuron & Herbicide & 100 & No \\
\hline Chlorantraniliprole & Insecticide & 100 & $?$ \\
\hline Imidacloprid & Pesticide/insecticide, endocrine disrupter & 1000 & $?$ \\
\hline Hidrasec & Antidiarrheal drug & 500 & $?$ \\
\hline Sunset yellow - FCF & Food additive & 500 & $?$ \\
\hline
\end{tabular}


2,3-DCP

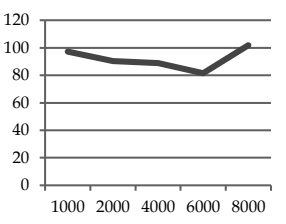

Arsenobetaine

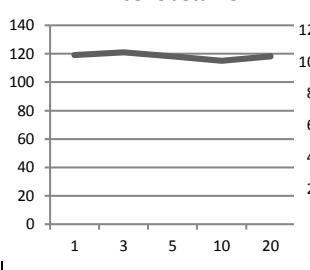

Chlopyrifos

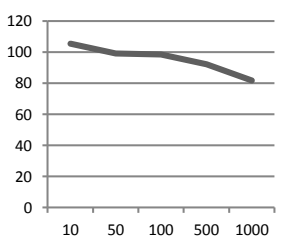

Hidrasec

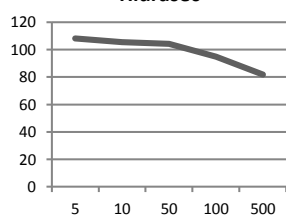

TBBPA

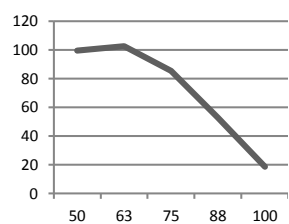

Aldicarb

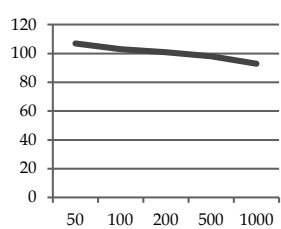

As203

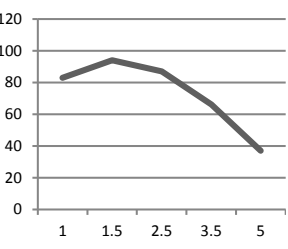

Chlorantraniliprole
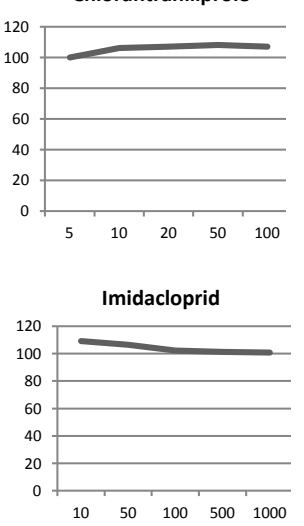

Zearalenone

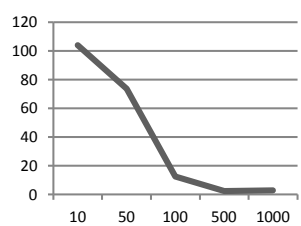

Ametoctradine

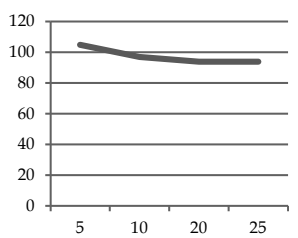

Benzo[e]pyrene

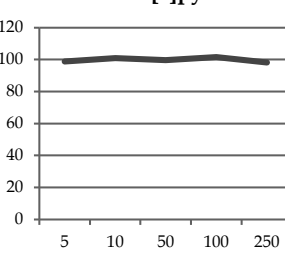

$\mathrm{CCL} 4$
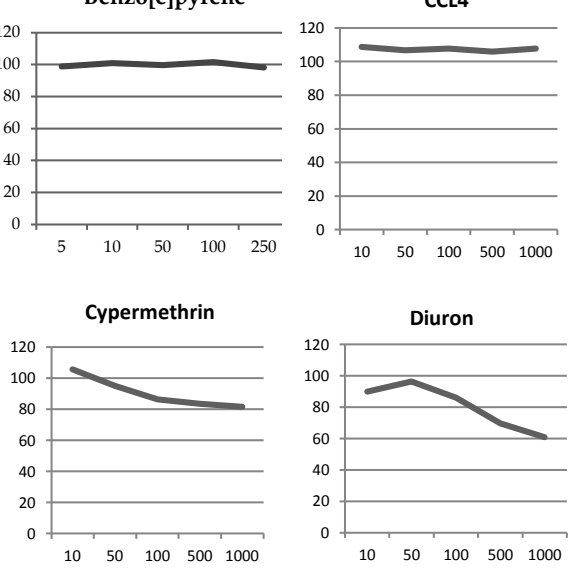

Sunset Yellow
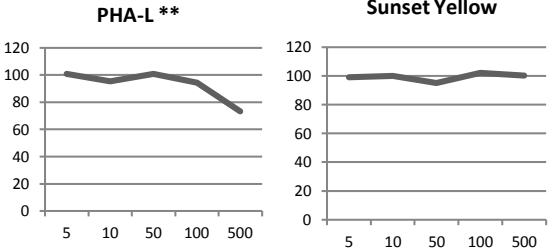

\section{Concentration $(\mu \mathrm{M})$}

FIG. 1. Average viability of compounds as measured by ATPlite assay. Results are based on at least 3 biological replicates of Jurkat cells and viability is corrected for solvent control DMSO. Average viability is shown on $\mathrm{Y}$-axis as percentage to DMSO, compound concentrations are present on $X$-axis in $\mu M$. Exceptions ${ }^{*} A n t i C D 3$ was dosed in $n g / \mu l$ and was corrected for lgG control. ${ }^{* *}$ PHA-L was dosed in $n g / m l$. 


\section{Immunotoxic Not Immunotoxic Unknown}

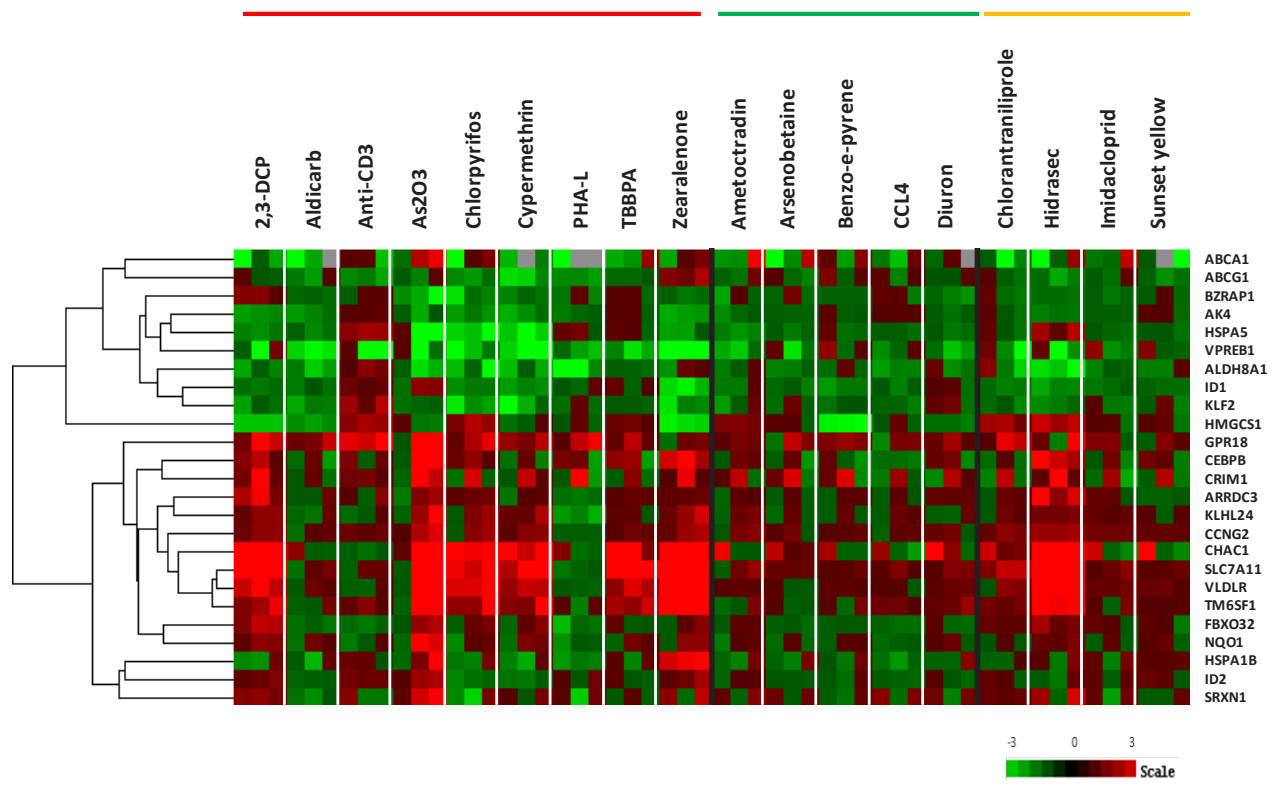

FIG. 2. Average expression of $N=25$ classifier genes in human Jurkat $T$ cells exposed to 18 different compounds. Values represent the average of technical duplicates. Each compound is tested in three independent biological replicates. The order of the genes was determined by hierarchical clustering. Red indicates that the particular gene is upregulated, green indicates a downregulation, grey represents missing values. Colour scale represents 2 log ratios vs. vehicle control.

TABLE 3. Functional themes in which the 25 classifiers are involved.

\begin{tabular}{c|c}
\hline Category & Genes \\
\hline Regulation of cell cycle and apoptosis & CCNG2, CRIM1, CHAC1, HSPA1B, ID1, ID2 \\
Metabolism and transport of cholesterol & HMGCS1, ABCA1, ABCG1 \\
Cellular signal transduction & BZRAP1, ARRDC3, CRIM1, GPR18, KLHL24, CHAC1 \\
Cellular stress responses & KLF2, CHAC1, HSPA1B, HSPA5, NQO1, SRXN1, AK4, SLC7A11, VLDLR, \\
Immune regulation & TM6SF1, ABCA1, ABCG1 \\
Protein metabolism & FLPX32, CRIM1, AK4 \\
Retinoic acid metabolism & ALDH8A1 ID1, ID2, CEBPB \\
Regulation of transcription & ID1, ID2 \\
\hline
\end{tabular}




\section{ToxPi analyses}

We categorized the 25 candidate classifier genes into eight functional themes, based on the major GO processes and molecular functions that these genes are involved in (Table 3). The ToxPi tool was then used to gain insight into the modes of action of the compounds. Results of this analysis are shown in pie graphs in Figure 3. A first observation is that all compounds affected protein metabolism to an almost similar degree. Based on similarities in ToxPi results, the compounds could be classified into three groups. The first group was formed by zearalenone, Hidrasec and $\mathrm{As}_{2} \mathrm{O}_{3}$ which all affected genes in cellular signal transduction and cellular stress responses (panel A). Next to these similarities, zearalenone affects immune regulation and Hidrasec affects retinoic acid metabolism which are processes that are hardly affected by the other two compounds.

Two compounds with almost similar pie graph, but no similarities in structure are 2,3-DCP and cypermethrin (panel B). Both compounds affect protein metabolism and cholesterol metabolism \& transport. In addition, cypermethrin affects immune regulation and transcription regulation that are not or to a lesser extent regulated by 2,3-DCP. ToxPi analysis of four compounds, chlorpyrifos, benzo-epyrene, aldicarb and anti-CD3, resulted in similar pie graphs (panel C). These four compounds all affect cholesterol metabolism and transport, protein metabolism and transcription regulation, and to a lesser extent retinoic acid metabolism and immune regulation.

\begin{tabular}{c|c|c}
\hline Color & Category & $\begin{array}{c}\text { Genes } \\
\text { (N) }\end{array}$ \\
\hline$\square$ & $\begin{array}{c}\text { Regulation cell } \\
\text { cycle \& apoptosis } \\
\text { Cholesterol } \\
\text { metabolism\& } \\
\text { transport } \\
\text { Cellular signal } \\
\text { transduction }\end{array}$ & 6 \\
$\square$ & $\begin{array}{c}\text { Cellular stress } \\
\text { responses }\end{array}$ & 12 \\
$\square$ & $\begin{array}{c}\text { Immune regulation } \\
\text { Protein metabolism } \\
\text { Retinoic acid } \\
\text { metabolism } \\
\text { Regulation } \\
\text { transcription }\end{array}$ & 3 \\
\hline
\end{tabular}

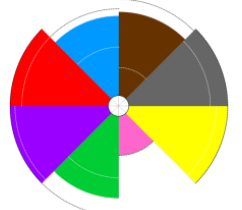

Zearalenone

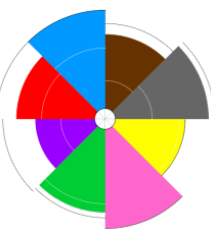

Hidrasec

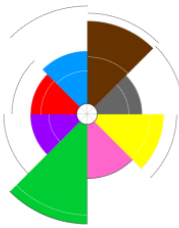

2,3-DCP

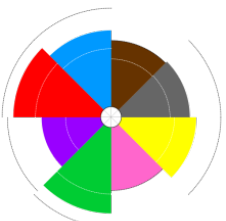

As203

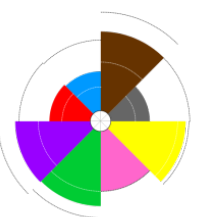

Cypermethrin
A

B 


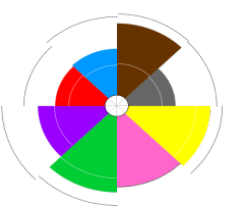

Chlorpyrifos

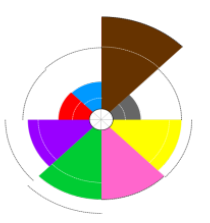

Aldicarb

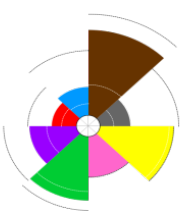

C
Benzo-e-pyrene

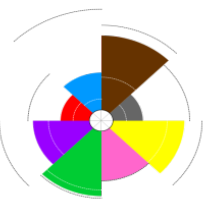

Anti-CD3

FIG. 3. Visualization of the effects of example compounds on eight functional themes. The pie graphs visualize the effects of each compound on the functional themes as listed in the table (left side of Fig. 3A). Each pie represents one compound. Each piece of the pie represents one single functional theme. The eight functional themes were weighted equally, so the graphic widths of all slices are equal. The size of each piece (the distance to the centre, between 0 to 1) shows the ToxPi score that was calculated by the ToxPi software. Pie graphs are shown for the following compounds: (A) Zearalenone, Hidrasec and As2O3. (B) 2.3-DCP and cypermethrin. (C) Chlorpyrifos, benzo-e-pyrene, aldicarb and anti-CD3.

\section{Effects on mode of action (MOA) signature genes}

In order to validate the MOAs identified with the present study, we also performed qRT-PCR for all compounds on eight additional genes with known functions. An overview of the primers for these genes is given in Table 4. Seven compounds resulted in significantly altered expression of at least one of the eight genes. The results of the PCR experiments for these seven compounds are visualised as bar graphs in Figure 4. PHA-L upregulated the early T cell activation marker CD69 (Ziegler, et al. 1994), which corresponds to the mechanism of action of T cell activation. CD69 was also upregulated by TBBPA which indicates that this compound induces $T$ cell activation as well. TBBPA also induced expression of HERPUD1, a known marker for an induced unfolded protein response that may lead to ER stress (Hori, et al. 2004). Other compounds that increased the expression of HERPUD1 and are thus likely to induce ER stress are Hidrasec and 2,3-DCP. 2,3-DCP downregulated SQLE, which is involved in cholesterol metabolism and transport (Bonne, et al. 2002). This decrease of cholesterol metabolism support the biological interpretation based on the 25 classifier genes for 2,3-DCP in Figure 3A. SQLE was also downregulated by anti-CD3 and zearalenone, and upregulated by chlorpyrifos and hidrasec which is in agreement with the results shown in Figures 3A and 3B. SGK1 codes for a protein that is involved in cell growth, survival, migration and cellular stress response (Amato, et al. 2009). SGK1 was upregulated by anti-CD3 and 
chlorpyrifos. Hidrasec downregulated LGALS1, a gene that is involved in cell cycle and apoptosis, which also supports the outcome of the ToxPi analysis. LGALS1 was also downregulated by chlorpyrifos and upregulated by anti-CD3, however, regulation of cell cycle and apoptosis was not obvious from the pie-graphs for these compounds (Fig.3).

Of the compounds that were immunotoxic based on the classifiers, As2O3, aldicarb and cypermethrin did not affect any of the additional genes. Furthermore, benzo-e-pyrene, the false positive as based on the 25 identifiers had also no effect on these additional genes. Moreover, the compounds selected as nonimmunotoxic did not change the expression of any of the eight additional genes.

TABLE 4. Overview of eight gene markers for investigating possible modes of action

\begin{tabular}{|c|c|c|c|c|}
\hline Gene Symbol & Description & Process & mRNA accession & Taqman assay \\
\hline CD69 & Cluster of Differentiation 69 & T cell activation & NM_001781 & hs00934033_m1 \\
\hline DDIT3 & $\begin{array}{l}\text { DNA damage inducible } \\
\text { transcript } 3\end{array}$ & $\begin{array}{l}\text { DNA damage, } \\
\text { apoptosis }\end{array}$ & NM_001195053 & Hs00358796_g1 \\
\hline DDIT4 & $\begin{array}{c}\text { DNA damage inducible } \\
\text { transcript } 4\end{array}$ & Immune regulation & NM_019058 & Hs01111686_g1 \\
\hline HERPUD1 & $\begin{array}{l}\text { Homocysteine-responsive } \\
\text { endoplasmic reticulum- } \\
\text { resident ubiquitin-like domain } \\
\text { member } 1\end{array}$ & $\begin{array}{l}\text { ER stress / Unfolded } \\
\text { protein response }\end{array}$ & NM_001010989 & Hs01124269_m1 \\
\hline LGALS1 & Galectin-1 & Apoptosis, cell cycle & NM_002305 & Hs00355202_m1 \\
\hline SGK1 & $\begin{array}{l}\text { Serine/threonine-protein } \\
\text { kinase } 1\end{array}$ & $\begin{array}{l}\text { Regulation of } \\
\text { transcription }\end{array}$ & NM_001143676 & Hs00985033_g1 \\
\hline SQLE & squalene epoxidase & $\begin{array}{l}\text { Cholesterol } \\
\text { homeostasis }\end{array}$ & NM_003129 & Hs01123768_m1 \\
\hline TRIB3 & Tribbles homolog 3 & Apoptosis, ER stress & NM_021158 & hs01082394_m1 \\
\hline
\end{tabular}



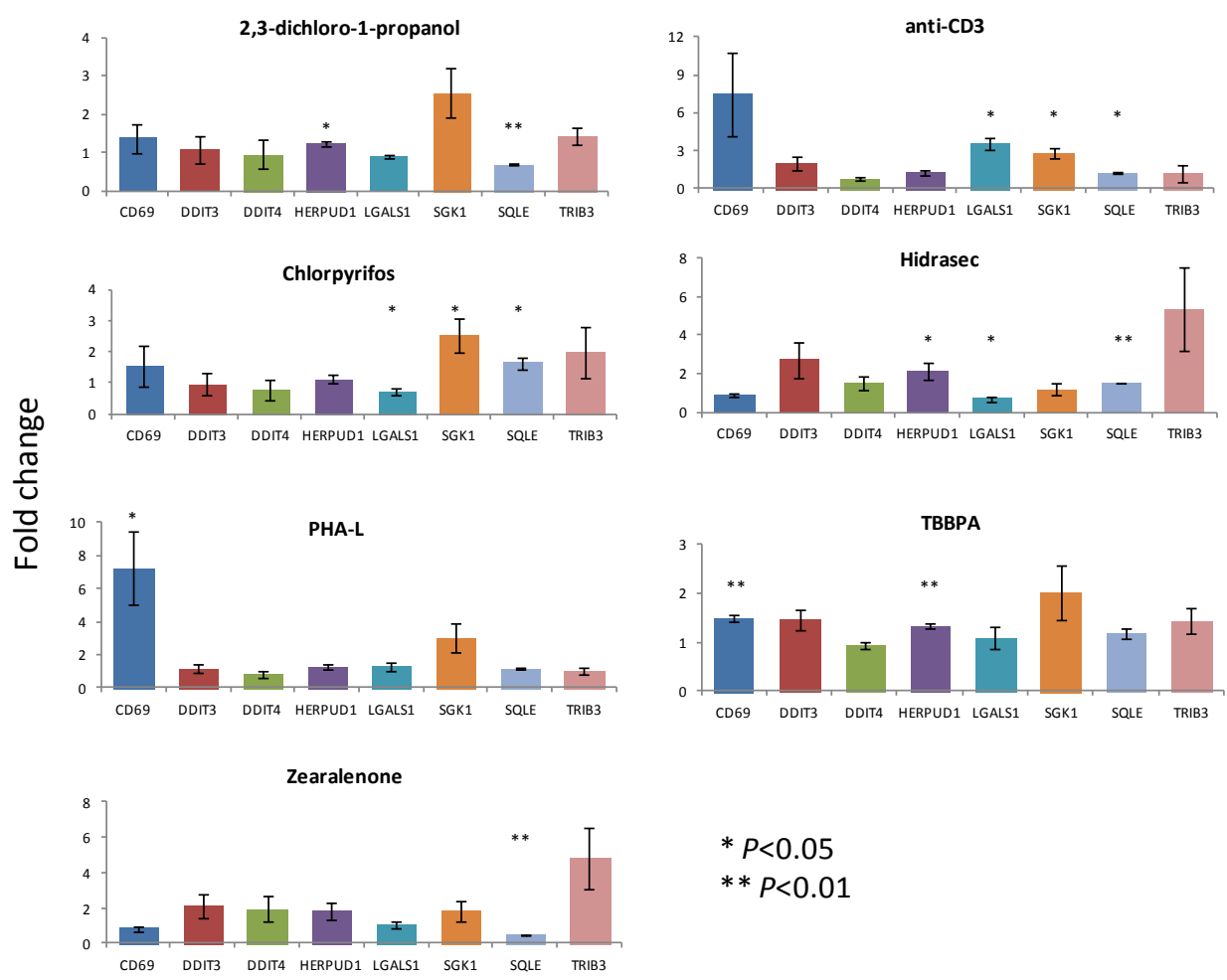

$$
\begin{aligned}
& * P<0.05 \\
& * * P<0.01
\end{aligned}
$$

FIG. 4. Average expression of genes indicating modes of action. Performance of eight qRT-PCR genes that are involved in $T$ cell activation and proliferation (CD69 and LGALS1), DNA damage and apoptosis (DDIT3, DDIT4, TRIB3), ER stress induction (HERPUD1), cellular stress response (SGK1), and sterol biosynthesis (SQLE). Data is based on three independent biological replicates. ${ }^{*} P<0.05$ and ${ }^{\star \star} P<0.01$ compared to control (Student's $T$ test). Y-axis: Fold change as corrected by DMSO and reference genes.

\section{Discussion}

The identification and validation of the 25 classifier genes was performed previously on a training set of 36 immunotoxic and 8 nonimmunotoxic compounds, and an external verification set of 17 immunotoxic and 3 nonimmunotoxic compounds (Shao, et al. 2014). The classifiers were chosen in such a way that they covered a broad range of molecular mechanisms of action.

The present study was designed for two purposes, being (1) further validation of the 25 classifiers with additional immunotoxicants and nonimmunotoxic control compounds, and (2) application of the classifiers to screen a group of compounds for which human immunotoxicity has not yet been fully established. For the first purpose, we exposed Jurkat cells to nine known immunotoxicants and five 
known nonimmunotoxicants. These compounds included new compound classes that were not tested before, such as the polybrominated flame retardant TBBPA and the biological anti-CD3. The four compounds selected for the second purpose were the neonicotinoid imidacloprid, the insecticide chlorantraniliprole, the antidiarrheal drug hidrasec and the food additive sunset yellow FCF. In this second round of validation, the 25 classifiers generated a higher sensitivity ( 100 vs. $88 \%$ ), specificity ( 80 vs. $67 \%$ ) and overall accuracy ( 93 vs. $85 \%$ ) than obtained in our previous validation (Shao, et al. 2014). When results of our present study were combined with those of Shao et. al. (2014), the sensitivity, specificity and accuracy would be 91,71 , and $85 \%$, respectively. In addition, we show that the 25 classifiers correctly predicted the immunotoxicity of compounds from classes not tested before in the Jurkat assay including TBBPA, anti CD3, chlorpyrifos, PHA-L and 2,3-DCP.

This current exercise resulted in only one false positive (benzo-e-pyrene). Benzo-e-pyrene is a structural analogue of benzo-a-pyrene which is a known immunotoxicant (Boorman, et al. 1982). Although benzo-e-pyrene is used as a nonimmunotoxic control for polyaromatic hydrocarbon (PAH) exposure (Davila, et al. 1999), some studies indicate slight immunotoxic effects of benzo-e-pyrene (Davila, et al. 1996, Krieger, et al. 1995). The only gene that was regulated by benzo-e-pyrene was HMGCS1, which is regulated in cholesterol metabolism and transport (Horton, et al. 2002).

We investigated possible mode of actions of the test compounds by using ToxPi analysis based on the expression of the 25 classifier genes. In addition, we performed qRT-PCR on eight additional genes, in order to support the putative MOAs we identified with the 25 classifiers. None of the nonimmunotoxic compounds in the present study affected the expression of the eight additional genes tested. We determined the fold changes in expression of these eight additional genes regarding the 31 compounds of the previous study (Shao, et al. 2014). Also in this study the nonimmunotoxic compounds did not alter the expression of any of the eight additional genes (results not shown).

TBBPA is known to induce oxidative stress followed by DNA damage (Choi, et al. 2011) and stimulate immune cells (Koike, et al. 2013). In our study, TBBPA induced expression of T cell activation marker CD69, which corresponds to the results of Koike et. al., but also upregulated ER stress/UPR gene HERPUD1 (Hori, et al. 2004), which is a new finding (Fig. 4). In addition, TBBPA upregulated the proapoptosis marker CHAC1 (Mungrue, et al. 2009) almost 4 times. Furthermore, TBBPA is known to induce cell death (in Sertoli cells) by affecting calcium homeostasis (Ogunbayo, et al. 2008). Disruption of calcium homeostasis in T cells may lead to ER stress (Gwack, et al. 2007, Katika, et al. 2012). Cells that do not recover from ER stress will undergo apoptotic events.

Induction of ER stress is not limited to immune cells, however, immune cells are more sensitive to ER stress inducing compounds such as deoxynivalenol and tributyltin oxide. Previously we demonstrated that $T$ cells are more sensitive because of the fact that ER stress leads to a leakage of calcium ions from the ER, leading to a calcium signal that in turn generates a T cell activation response, which is a measure for immunotoxicity (Katika, et al. 2012, Katika, et al. 2012, Schmeits, et al. 2013). Although 
ER stress may occur in other cells as well (at higher concentrations than in immune cells), in those cells this will not lead to a $\mathrm{T}$ cell activation response.

Currently, the mechanism by which the mycotoxin zearalenone exerts its immunotoxicity is not clear. We show that zearalenone affects genes in cellular stress responses, cholesterol metabolism/transport, immune regulation, cellular signal transduction, cell cycle and apoptosis and transcription. In addition, zearalenone downregulated one of the eight additional genes tested, SQLE, which is involved in cholesterol metabolism. The fact that some of the immunotoxic compounds affect genes involved in cholesterol metabolism might very well reflect the cellular response to disruption of cholesterol homeostasis.

Based on our results obtained with the 25 classifier genes, 2,3-DCP mainly affects cholesterol metabolism and transport and protein metabolism. PCR analysis of additional genes, such as HERPUD1 and SQLE, supported these findings. A previous study indicated that 2,3-DCP inhibits T cell activation and downregulates activation of transduction pathways in vitro (mouse $T$ cells purified from splenocytes) and mice in vivo (Lu, et al. 2013). However, 2,3-DCP did not significantly affect expression of the T cell activation marker CD69 in the present study. This is most likely caused by the fact that Jurkat cells generally were cultured without stimulus (PHA/LPS). An inhibition of $T$ cell activation is therefore hard to detect.

In the present study chlorpyrifos induces cellular stress responses and cholesterol biosynthesis in Jurkat T cells. Previous studies found that chlorpyrifos induced oxidative stress (Ambali, et al. 2007, Gultekin, et al. 2001). Also, chlorpyrifos induces hyperlipidaemia, a state in which cholesterol synthesis is elevated in rats (Acker and Nogueira 2012) which is in line with the results obtained in our study.

Of the four compounds that might be immunotoxic, chlorantraniliprole and Hidrasec were classified as immunotoxic, and the other two (imidacloprid and sunset yellow) as non immunotoxic, as based on the genetic markers used in this Jurkat study. Imidacloprid has been tested immunotoxic in rats and mice in vivo (Badgujar, et al. 2013, Gawade, et al. 2013). No data was available on human immune cells. Imidacloprid does not affect any of the 25 classifiers or eight additional genes tested in this study. Sunset yellow is known to affect monocytes but no other blood cells in mice in vivo (Hashem, et al. 2010). Apparently, sunset yellow is also not toxic for human Jurkat T cells. This result points to the limitation of the current assay that compounds targeting types of immune cells other than $T$ cells might be not detected. Both chlorantraniliprole and Hidrasec were assessed nonimmunotoxic in rodent immunotoxicity studies. The discrepancy between the outcome of the animal studies and the current Jurkat study with these compounds might be due to inter-species differences. It is generally recognised that the predictability of rodent studies for human toxicity is rather low (Shanks, et al. 2009). For this reason, the US National Research Council (2007), and other organisations, developed a future vision in which animal models are being replaced by human in vitro models. Chlorantraniliprole is suspected to act on release of calcium from intracellular calcium stores (Lahm, et al. 2005) that is known to lead to T cell activation in T cells (Gwack, et al. 2007, Katika, et al. 2012). 
Chlorantraniliprole mainly affects cellular stress and signal response pathways in our present experiments. One of the genes upregulated by chlorantraniliprole is SLC7A11 (alias XCT). SLC7A11 represents the rate limiting step of glutathione synthesis, and overexpression of SLC7A11 reduces the need for calcium for early cell cycle progression in early cell cycle (Lastro, et al. 2008).

In conclusion we have proven the good prediction performance of Jurkat $\mathrm{T}$ cell screening system based on 25 classifiers when challenged by new classes of immunotoxicants. The sensitivity, specificity and accuracy were even slightly higher in the present study as compared to the previous study. In addition to its use for predicting direct immunotoxicity, this set of genes can be exploited to give indications for the modes of action of compounds. This system however may not detect compounds that exert immunotoxic action on other immune cells than T cells, which likely is the case for sunset yellow. Therefore, the Jurkat $T$ cell line is a valuable model for immunotoxicity screening not on itself but as part of an integrated testing strategy.

\section{Acknowledgements}

This study was financially supported by the Netherlands Genomics Initiative, the Netherlands Toxicogenomics Centre (grant 05060510). 


\section{References}

2007. Toxicity Testing in the 21st Century: A Vision and a Strategy. The National Academies Press Abbes S, Ben Salah-Abbes J, Sharafi H, Oueslati R, Noghabi KA. 2013. Lactobacillus paracasei BEJ01 prevents immunotoxic effects during chronic zearalenone exposure in Balb/c mice. Immunopharmacol Immunotoxicol 35:341-348. DOI: 10.3109/08923973.2013.772194.

Acker Cl, Nogueira CW. 2012. Chlorpyrifos acute exposure induces hyperglycemia and hyperlipidemia in rats. Chemosphere 89:602-608. DOI: 10.1016/j.chemosphere.2012.05.059.

Amato R, D'Antona L, Porciatti G, Agosti V, Menniti M, Rinaldo C, Costa N, Bellacchio E, Mattarocci S, Fuiano G, Soddu S, Paggi MG, Lang F, Perrotti N. 2009. Sgk1 activates MDM2-dependent p53 degradation and affects cell proliferation, survival, and differentiation. $J$ Mol Med (Berl) 87:1221-1239. DOI: 10.1007/s00109-009-0525-5.

Ambali S, Akanbi D, Igbokwe N, Shittu M, Kawu M, Ayo J. 2007. Evaluation of subchronic chlorpyrifos poisoning on hematological and serum biochemical changes in mice and protective effect of vitamin C. J Toxicol Sci 32:111-120.

Badgujar PC, Jain SK, Singh A, Punia JS, Gupta RP, Chandratre GA. 2013. Immunotoxic effects of imidacloprid following 28 days of oral exposure in BALB/c mice. Environ Toxicol Pharmacol 35:408418. DOI: 10.1016/j.etap.2013.01.012.

Birnbaum LS, Staskal DF. 2004. Brominated flame retardants: cause for concern? Environ Health Perspect 112:9-17.

Bonne AC, den Bieman MG, Gillissen GF, van Lith HA, van Zutphen LF. 2002. Chromosomal localization of genes involved in biosynthesis, metabolism or transport of cholesterol in the rat. Cytogenet Genome Res 97:183-186. DOI: 66606.

Boorman GA, Luster MI, Dean JH, Campbell ML. 1982. Assessment of myelotoxicity caused by environmental chemicals. Environ Health Perspect 43:129-135.

Borak J, Hosgood HD. 2007. Seafood arsenic: implications for human risk assessment. Regul Toxicol Pharmacol 47:204-212. DOI: 10.1016/j.yrtph.2006.09.005.

Choi JS, Lee YJ, Kim TH, Lim HJ, Ahn MY, Kwack SJ, Kang TS, Park KL, Lee J, Kim ND, Jeong TC, Kim SG, Jeong HG, Lee BM, Kim HS. 2011. Molecular Mechanism of Tetrabromobisphenol A (TBBPA)-induced Target Organ Toxicity in Sprague-Dawley Male Rats. Toxicol Res 27:61-70. DOI: 10.5487/TR.2011.27.2.061.

Davila DR, Lane JL, Lauer FT, Burchiel SW. 1999. Protein tyrosine kinase activation by polycyclic aromatic hydrocarbons in human HPB-ALL T cells. J Toxicol Environ Health A 56:249-261. DOI: 10.1080/009841099158097.

Davila DR, Romero DL, Burchiel SW. 1996. Human T cells are highly sensitive to suppression of mitogenesis by polycyclic aromatic hydrocarbons and this effect is differentially reversed by alphanaphthoflavone. Toxicol Appl Pharmacol 139:333-341. DOI: 10.1006/taap.1996.0173.

Dunier M, Siwicki AK, Demael A. 1991. Effects of organophosphorus insecticides: effects of trichlorfon and dichlorvos on the immune response of carp (Cyprinus carpio). III. In vitro effects on lymphocyte 
proliferation and phagocytosis and in vivo effects on humoral response. Ecotoxicol Environ Saf 22:7987.

EC. 2006. Regulation (EC) No 1907/2006 of the European Parliament and of the Council of 18 December 2006 concerning the Registration, Evaluation, Authorisation and Restriction of Chemicals (REACH), establishing a European Chemicals Agency, amending Directive 1999/45/EC and repealing Council Regulation (EEC) No 793/93 and Commission Regulation (EC) No 1488/94 as well as Council Directive 76/769/EEC and Commission Directives 91/155/EEC, 93/67/EEC, 93/105/EC and 2000/21/EC. EC (ed). OJ L; 1-849

diuron Immunotoxicity. http://apps.echa.europa.eu/registered/data/dossiers/DISS-9d864497-d81a5ced-e044-00144f67d249/AGGR-ad88c783-e215-4ee3-8ac8-7fe393b61600_DISS-9d864497-d81a5ced-e044-00144f67d249.html

Eisen MB, Spellman PT, Brown PO, Botstein D. 1998. Cluster analysis and display of genome-wide expression patterns. Proc Natl Acad Sci U S A 95:14863-14868.

EMEA. 2006. NOTE FOR GUIDANCE ON IMMUNOTOXICITY STUDIES FOR HUMAN PHARMACEUTICALS. In ICH Topic S 8 Immunotoxicity Studies for Human Pharmaceuticals, Agency EM (ed). 13

EPA. 2013. Part 158 Toxicology Data Requirements: Guidance for Neurotoxicity Battery, Subchronic Inhalation, Subchronic Dermal and Immunotoxicity Studies. Office of Pesticide Programs USEPA (ed). 11

FDA. 2002. Guidance for Industry - Immunotoxicology Evaluation of Investigational New Drugs. (CDER) USDoHaHSFaDACfDEaR (ed). ICCVAM; 35

Fernandez-Riejos P, Goberna R, Sanchez-Margalet V. 2008. Leptin promotes cell survival and activates Jurkat $\mathrm{T}$ lymphocytes by stimulation of mitogen-activated protein kinase. Clin Exp Immunol 151:505-518. DOI: 10.1111/j.1365-2249.2007.03563.x.

Fischer AM, Mercer JC, Iyer A, Ragin MJ, August A. 2004. Regulation of CXC chemokine receptor 4mediated migration by the Tec family tyrosine kinase ITK. J Biol Chem 279:29816-29820. DOI: 10.1074/jbc.M312848200.

Gawade L, Dadarkar SS, Husain R, Gatne M. 2013. A detailed study of developmental immunotoxicity of imidacloprid in Wistar rats. Food Chem Toxicol 51:61-70. DOI: 10.1016/j.fct.2012.09.009.

Gultekin F, Delibas N, Yasar S, Kilinc I. 2001. In vivo changes in antioxidant systems and protective role of melatonin and a combination of vitamin $\mathrm{C}$ and vitamin $\mathrm{E}$ on oxidative damage in erythrocytes induced by chlorpyrifos-ethyl in rats. Arch Toxicol 75:88-96.

Gwack Y, Feske S, Srikanth S, Hogan PG, Rao A. 2007. Signalling to transcription: store-operated Ca2+ entry and NFAT activation in lymphocytes. Cell Calcium 42:145-156. DOI: 10.1016/j.ceca.2007.03.007.

Hashem MM, Atta AH, Arbid MS, Nada SA, Asaad GF. 2010. Immunological studies on Amaranth, Sunset Yellow and Curcumin as food colouring agents in albino rats. Food Chem Toxicol 48:15811586. DOI: 10.1016/j.fct.2010.03.028. 
Hori O, Ichinoda F, Yamaguchi A, Tamatani T, Taniguchi M, Koyama Y, Katayama T, Tohyama M, Stern DM, Ozawa K, Kitao Y, Ogawa S. 2004. Role of Herp in the endoplasmic reticulum stress response. Genes Cells 9:457-469. DOI: 10.1111/j.1356-9597.2004.00735.x.

Horton JD, Goldstein JL, Brown MS. 2002. SREBPs: activators of the complete program of cholesterol and fatty acid synthesis in the liver. J Clin Invest 109:1125-1131. DOI: 10.1172/JCI15593. Institoris L, Siroki O, Desi I, Lesznyak J, Serenyi P, Szekeres E, Petri I. 1998. Extension of the protocol of OECD guideline 407 (28-day repeated dose oral toxicity test in the rat) to detect potential immunotoxicity of chemicals. Hum Exp Toxicol 17:206-211.

Ishiguro K, Xavier R. 2004. Homer-3 regulates activation of serum response element in T cells via its EVH1 domain. Blood 103:2248-2256. DOI: 10.1182/blood-2003-08-2671.

Jin Y, Zheng S, Fu Z. 2011. Embryonic exposure to cypermethrin induces apoptosis and immunotoxicity in zebrafish (Danio rerio). Fish Shellfish Immunol 30:1049-1054. DOI: 10.1016/j.fsi.2011.02.001.

Katika MR, Hendriksen PJ, de Ruijter NC, van Loveren H, Peijnenburg A. 2012. Immunocytological and biochemical analysis of the mode of action of bis (tri-n-butyltin) tri-oxide (TBTO) in Jurkat cells. Toxicol Lett 212:126-136. DOI: 10.1016/j.toxlet.2012.05.010.

Katika MR, Hendriksen PJ, Shao J, van Loveren H, Peijnenburg A. 2012. Transcriptome analysis of the human $\mathrm{T}$ lymphocyte cell line Jurkat and human peripheral blood mononuclear cells exposed to deoxynivalenol (DON): New mechanistic insights. Toxicol Appl Pharmacol 264:51-64. DOI: 10.1016/j.taap.2012.07.017.

Koike E, Yanagisawa R, Takigami H, Takano H. 2013. Brominated flame retardants stimulate mouse immune cells in vitro. J Appl Toxicol 33:1451-1459. DOI: 10.1002/jat.2809.

Krieger JA, Davila DR, Lytton J, Born JL, Burchiel SW. 1995. Inhibition of sarcoplasmic/endoplasmic reticulum calcium ATPases (SERCA) by polycyclic aromatic hydrocarbons in HPB-ALL human T cells and other tissues. Toxicol Appl Pharmacol 133:102-108. DOI: 10.1006/taap.1995.1131.

Lahm GP, Selby TP, Freudenberger JH, Stevenson TM, Myers BJ, Seburyamo G, Smith BK, Flexner L, Clark CE, Cordova D. 2005. Insecticidal anthranilic diamides: a new class of potent ryanodine receptor activators. Bioorg Med Chem Lett 15:4898-4906. DOI: 10.1016/j.bmcl.2005.08.034.

Lankveld DP, Van Loveren H, Baken KA, Vandebriel RJ. 2010. In vitro testing for direct immunotoxicity: state of the art. Methods Mol Biol 598:401-423. DOI: 10.1007/978-1-60761-401-2_26. Lastro M, Kourtidis A, Farley K, Conklin DS. 2008. xCT expression reduces the early cell cycle requirement for calcium signaling. Cell Signal 20:390-399. DOI: 10.1016/j.cellsig.2007.10.030.

Lu J, Huang G, Zhang S, Song B, Wang Z, Xu L, Zhang S, Guan S. 2013. The inhibition of 2,3dichloro-1-propanol on $T$ cell in vitro and in vivo. Int Immunopharmacol 17:321-328. DOI: 10.1016/j.intimp.2013.06.026.

Mirkin IR, Anderson HA, Hanrahan L, Hong R, Golubjatnikov R, Belluck D. 1990. Changes in Tlymphocyte distribution associated with ingestion of aldicarb-contaminated drinking water: a follow-up study. Environ Res 51:35-50. 
Mitsuishi Y, Falkenrodt A, Urlacher A, Tongio MM, Mayer S. 1986. New human MHC class I antigens segregating with HLA-A antigens detected on some lymphocyte subpopulations. Hum Immunol 15:175-197.

Mungrue IN, Pagnon J, Kohannim O, Gargalovic PS, Lusis AJ. 2009. CHAC1/MGC4504 is a novel proapoptotic component of the unfolded protein response, downstream of the ATF4-ATF3-CHOP cascade. J Immunol 182:466-476.

Noworyta-Glowacka J, Bankowski R, Siennicka J, Wiadrowska B, Ludwicki JK. 2012. Influence of chlorpyrifos on the profile of subpopulations of immunoactive cells and their phagocytic activity in an experimental in vivo model. Ann Agric Environ Med 19:483-486.

Ogunbayo OA, Lai PF, Connolly TJ, Michelangeli F. 2008. Tetrabromobisphenol A (TBBPA), induces cell death in TM4 Sertoli cells by modulating $\mathrm{Ca} 2+$ transport proteins and causing dysregulation of Ca2+ homeostasis. Toxicol In Vitro 22:943-952. DOI: 10.1016/j.tiv.2008.01.015.

Pullen S, Boecker R, Tiegs G. 2003. The flame retardants tetrabromobisphenol A and tetrabromobisphenol A-bisallylether suppress the induction of interleukin-2 receptor alpha chain (CD25) in murine splenocytes. Toxicology 184:11-22.

Reif DM, Sypa M, Lock EF, Wright FA, Wilson A, Cathey T, Judson RR, Rusyn I. 2013. ToxPi GUI: an interactive visualization tool for transparent integration of data from diverse sources of evidence. Bioinformatics 29:402-403. DOI: 10.1093/bioinformatics/bts686.

Sakurai T, Kojima C, Ochiai M, Ohta T, Fujiwara K. 2004. Evaluation of in vivo acute immunotoxicity of a major organic arsenic compound arsenobetaine in seafood. Int Immunopharmacol 4:179-184. DOI: 10.1016/j.intimp.2003.11.004.

Schmeits PC, Katika MR, Peijnenburg AA, van Loveren H, Hendriksen PJ. 2013. DON shares a similar mode of action as the ribotoxic stress inducer anisomycin while TBTO shares ER stress patterns with the ER stress inducer thapsigargin based on comparative gene expression profiling in Jurkat T cells. Toxicol Lett, 10.1016/j.toxlet.2013.11.005. DOI: 10.1016/j.toxlet.2013.11.005.

Schmeits PC, Volger OL, Zandvliet ET, van Loveren H, Peijnenburg AA, Hendriksen PJ. 2013. Assessment of the usefulness of the murine cytotoxic T cell line CTLL-2 for immunotoxicity screening by transcriptomics. Toxicol Lett 217:1-13. DOI: 10.1016/j.toxlet.2012.12.005.

Shanks N, Greek R, Greek J. 2009. Are animal models predictive for humans? Philos Ethics Humanit Med 4:2. DOI: 10.1186/1747-5341-4-2.

Shao J, Berger LF, Hendriksen PJ, Peijnenburg AA, van Loveren H, Volger OL. 2014. Transcriptomebased functional classifiers for direct immunotoxicity. Arch Toxicol 88:673-689. DOI: 10.1007/s00204013-1179-1.

Shao J, Katika MR, Schmeits PC, Hendriksen PJ, van Loveren H, Peijnenburg AA, Volger OL. 2013. Toxicogenomics-based identification of mechanisms for direct immunotoxicity. Toxicol Sci 135:328346. DOI: 10.1093/toxsci/kft151.

Smialowicz RJ, Simmons JE, Luebke RW, Allis JW. 1991. Immunotoxicologic assessment of subacute exposure of rats to carbon tetrachloride with comparison to hepatotoxicity and nephrotoxicity. Fundam Appl Toxicol 17:186-196. 
Thomas PT, Ratajczak HV, Eisenberg WC, Furedi-Machacek M, Ketels KV, Barbera PW. 1987. Evaluation of host resistance and immunity in mice exposed to the carbamate pesticide aldicarb. Fundam Appl Toxicol 9:82-89.

Weetall M, Digan ME, Hugo R, Mathew S, Hopf C, Tart-Risher N, Zhang J, Shi V, Fu F, HammondMcKibben D, West S, Brack R, Brinkmann V, Bergman R, Neville D, Jr., Lake P. 2002. T-cell depletion and graft survival induced by anti-human CD3 immunotoxins in human CD3epsilon transgenic mice. Transplantation 73:1658-1666.

White KL, Jr., McLoughlin CE, Auttachoat W, Smith MJ. 2012. Validation of the Candida albicans delayed-type hypersensitivity (DTH) model in the female $B(6) C(3) F(1)$ mouse for use in immunotoxicological investigations. $J$ Immunotoxicol 9:141-147. DOI: 10.3109/1547691X.2011.636768.

Yadav A, Kumar A, Tripathi A, Das M. 2013. Sunset yellow FCF, a permitted food dye, alters functional responses of splenocytes at non-cytotoxic dose. Toxicol Lett 217:197-204. DOI: 10.1016/j.toxlet.2012.12.016.

Ziegler SF, Ramsdell F, Alderson MR. 1994. The activation antigen CD69. Stem Cells 12:456-465.

DOI: $10.1002 /$ stem.5530120502. 



\section{CHAPTER 8}

Summary, General Discussion, Future Perspectives, and

Conclusions 
The aims of the present thesis are (1) identification of mechanisms of action that are involved in chemical-induced direct immunotoxicity; and (2) identification of functional biomarkers that can be used for the development of an in vitro predictive assay for direct immunotoxicity. In order to place the present thesis in the context of other related research, background information regarding the topic and the aims of the thesis were described in Chapter 1. It provides an overview of the immune system, a number of definitions and human cases that are involved in immunotoxicology, current in vivo and in vitro testing approaches for direct immunotoxicity, an introduction of toxicogenomics, and the state of art regarding the application of -omics based techniques in the field of immunotoxicology. In the light of the above mentioned objectives, the main body of the thesis (Chapter 2 to 7 ) was divided into two sections.

In Section I, the microarray technology was applied in order to evaluate the effects of a wide range of chemicals, including known direct immunotoxicants, chemicals structurally related to certain direct immunotoxicants, immunosuppressive drugs, and nonimmunotoxic control chemicals on the whole genome mRNA expression in the human T lymphoblastic cell line Jurkat (Chapter 2). This study allowed the identification of several common mechanisms of action that are involved in chemicalinduced direct immunotoxicity, and the assignment of mechanisms of action to several immunotoxicants for which the mechanistic insights were previously limited or lacking. The effects of a subset of chemicals were further assessed by protein phosphorylation profiling of Jurkat cells using an antibody array-based approach (Chapter 3). This resulted in the recognition of cell signaling pathways commonly affected by direct immunotoxicants. One of the mechanisms of action that was identified for several immunotoxicants, being modulation of cell migration, was further examined in an in vitro functional chemotaxis assay (Chapter 4). The results of this study indicated that several chemicals may exert their immunotoxic effects through inhibition of immune cell chemotaxis. In order to determine whether the pathways and processes affected in the Jurkat T-cell line would also be affected in human primary immune cells, whole genome mRNA expression profiling was performed on human peripheral blood mononuclear cells (PBMCs) after the treatment with the model immunotoxicant TBTO; and the results were compared to the gene expression profiling data of TBTOtreated Jurkat cells (Chapter 5).

In Section II, the generated transcriptomic data in Chapter $\mathbf{2}$ was subjected to classifier analysis, in order to identify the genes that can be used to distinguish between immunotoxic and nonimmunotoxic chemicals. The identified classifier genes were further challenged with two new sets of chemicals, which comprised new classes of immunotoxicants that had not been tested in the transcriptomic experiment and more nonimmunotoxic control chemicals (Chapters 6 and 7). Good prediction performance was revealed in both sets.

\section{Potential mechanisms of action underlying chemical-induced direct immunotoxicity}


Transcriptomic-based identification of mechanisms of action- the basis for the development of predictive assay

Recently, the application of immunotoxicogenomics in both in vivo and in vitro studies has led to improved mechanistic understanding for a limited number of direct immunotoxic chemicals, including the biocide TBTO and the mycotoxin DON. However, a wide survey study on the potential mechanisms of action of direct immunotoxicants is currently lacking. Therefore, in Chapter 2, the effects of a large variety of chemicals (in total 31 chemicals) on the transcriptome of the human T-cell line Jurkat was assessed. These chemicals include several environmental contaminants, for instance heavy metals, pesticides, biocides, and mycotoxins, immunosuppressive drugs, and nonimmunotoxic control chemicals, which cover a wide range of chemical structures and classes.

By applying pathway analysis on the transcriptomic data, a list of canonical pathways and Gene Ontology processes were identified to be commonly regulated by several immunotoxicants, including ER stress, oxidative stress, anti-apoptotic responses, cell cycle control, regulation of transcription and translation, and metabolism of cholesterol and lipids. In addition, processes like Notch signaling, NF$\mathrm{KB}$ signaling, and response to retinoic acid (RXR/RAR signaling) were found to be specifically affected by one or two of the immunotoxicants. Part of the biological processes that were identified has been described in previous immunotoxicogenomics studies with single or few compounds, which also aimed at identifying pathways/genes involved in immunotoxicity, including cellular stress responses (response to chemical stimulus, response to unfolded proteins, and response to oxidative stress) and regulation of NF-KB signaling (Hochstenbach et al., 2012; Hochstenbach et al., 2010; Vandebriel et al., 2010). Moreover, this study also revealed new processes that are potentially relevant for direct immunotoxicity, such as modulation of lipid metabolism, RXR/RAR signaling, and Notch signaling. Further investigation would be required to validate the relevance of these potential mechanisms for direct immunotoxicity.

Protein phosphorylation profiling identified the mTOR pathway as a common target for direct immunotoxicants

In order to better understand the mechanisms underlying chemical-induced immunotoxicity, it was decided to evaluate the effects of chemicals at post-translational level, particularly on the phosphorylation of kinases/ proteins. In Chapter 3, a subset of chemicals, including five immunotoxicants (i.e., lindane, ochratoxin A, TBTC, TBTO, and DON), two immunosuppressive drugs (rapamycin and mycophenolic acid), and two nonimmunotoxic control chemicals (urethane and mannitol), that have been studied in the transcriptomic experiment of Chapter $\mathbf{2}$ was selected for a protein phosphorylation profiling study in Jurkat cells using a receptor tyrosine kinase (RTK) antibody array. This study aimed at identifying at the proteome/ kinome level common mechanisms of action that are involved in chemical-induced direct immunotoxicity. Some kinases were found to be commonly regulated by several immunotoxicants and immunosuppressive drugs, with the largest 
effect observed for ribosomal protein S6 (PRS6). By performing flow cytometry and western blot experiments with the model immunotoxicant TBTO, the results of the antibody array couls be successfully verified. Since PRS6 is known as an important downstream effector of the mTORp70S6K-RPS6 pathway (Chung et al., 1992), further investigation into this pathway was carried out with TBTO and rapamycin; the latter one is known as a selective inhibitor of the kinase mTOR. The results showed that, unlike rapamycin which dephosphorylated both mTOR and p70S6K, TBTO dephosphorylated p70S6K but had no effect on the phosphorylation of mTOR. These data indicated that both TBTO and rapamycin inhibit the P70S6K pathway, but via different molecular mechanisms. This suggests that although this pathway was found to be commonly affected by immunotoxicants and immunosuppressive drugs, the primary molecular targets of different chemicals may lie at different levels of the pathway.

\section{Comparison of the transcriptome and protein phosphorylation data}

Since for a few immunotoxicants we have obtained data at both the transcriptome and phosphoproteome levels, it was of interest to examine whether these two types of data correlated with each other. Therefore, the comparison was done at pathway level for the model immunotoxicant TBTO in Chapter 3. The results showed that both transcriptome and protein phosphorylation data provided evidence that TBTO affected ribosome biogenesis and cell migration processes in Jurkat cells.

Functional assay confirming the mechanism of action identified by toxicogenomics-based approaches

In order to further validate the hypothesis generated on the basis of the transcriptomics and protein phosphorylation data of TBTO, an in vitro trans-well chemotaxis assay was set up using Jurkat cells and the chemokine CXCL12 (Chapter 4). The results of this experiment indicated that TBTO significantly inhibited the migration of Jurkat cells towards CXCL12, which confirmed the results from the transcriptome and protein phosphorylation experiments described in Chapters $\mathbf{2}$ and $\mathbf{3}$.

Since it is shown that the in vitro trans-well chemotaxis assay with CXCL12 is sensitive enough to detect the inhibitory effect of TBTO on the migration of Jurkat cells, this experiment was extended with more chemicals in order to identify other immunotoxicants with chemotaxis-modulating properties (Chapter 4). First, an in-depth analysis of the transcriptomic data generated in Chapter 2 was performed with focus on mRNA expression of genes that are known to be related with cell migration. This analysis led to the identification of seven chemicals, of which five were confirmed as chemotaxis inhibitor in the trans-well chemotaxis assay. Another set of six chemicals were also tested in the assay, in order to verify whether chemicals that were not indicated as chemotaxis modulators, based on their effects at the transcriptome level, would also be negative in the chemotaxis assay. However, one out of the six additional chemicals, being the immunosuppressive drug mycophenolic acid, also 
significantly inhibited the chemotaxis of Jurkat cells. This result indicated that CXCL12-mediated chemotaxis is regulated not only at the transcriptome level. Nevertheless, these data showed that some immunotoxicants may exert their immunotoxic effects through inhibition of CXCL12-mediated chemotaxis. Furthermore, the transcriptome and protein phosphorylation profiles of the chemotaxis inhibitors, which were generated in Chapters 2 and 3, indicated that the mechanisms responsible for this inhibitory effect are different for different immunotoxicants. Another interesting finding of this study is that, unlike primary T cells, the mTOR signaling pathway is not crucial for CXCL12-mediated chemotaxis of Jurkat cells. This conclusion was drawn on the basis of the observation that the immunosuppressive drug rapamycin, and the other two p70S6K pathway modulators that were identified in Chapter 3, being the mycotoxin DON and the pesticide lindane, did not affect the chemotaxis of Jurkat cells towards CXCL12. Therefore, it can be expected that the chemotaxis assay conducted with Jurkat cells could generate false negative results for those chemicals that affect chemotaxis through mTOR signaling.

\section{Effects of TBTO on human peripheral blood mononuclear cells (PBMCs)}

Since the above mentioned results were all obtained in a human T-cell line, it was of interest and biological relevance to examine whether similar effects can be observed in human primary cells. Therefore, in Chapter 5, freshly isolated human peripheral blood mononuclear cells (PBMCs) were exposed to the model immunotoxicant TBTO for 6 and $24 \mathrm{~h}$, and then subjected to transcriptomic analysis.

Analysis of the microarray data of TBTO treated PBMCs revealed that the biological processes such as ER stress, oxidative stress, NF-KB signaling, protein synthesis, and mitochondria function were affected by TBTO in human PBMCs. These processes were also found to be affected by TBTO in Jurkat cells, as shown in Chapter $\mathbf{2}$ and 3, which indicated good correlation between the two in vitro systems. In addition, TBTO downregulated the genes that are related to lymphocyte subsets ( $T, B$, and NK cells), monocytes, dendritic cells, and granulocytes. These results are logical since PBMCs contain a mixture of different immune cell populations. In general, PBMCs isolated from human buffy coat are known to comprise $75 \% \mathrm{~T}$ lymphocytes, $16 \% \mathrm{~B}$ lymphocytes, $7 \%$ NK cells, and small amounts of basophils and dendritic cells. Regarding gene expression, each cell type has its own characteristic. Therefore, a number of immune-related processes were found to be affected in TBTOtreated PBMCs, but not in Jurkat cells.

\section{Functional biomarkers for the development of predictive assay}

Identification and first round of validation of the biomarkers 
Providing insights into the mechanisms of action of chemicals is obviously a big advantage of toxicogenomics, as compared to traditional animal-based toxicological studies. In addition, it has been demonstrated that gene expression profiling can also be a useful tool for the characterization of structural and functional chemical classes and the identification of new biomarkers for toxicity endpoints (Tugwood et al., 2003; Waters and Fostel, 2004). Therefore, in Chapter 6, the transcriptomic data generated in Chapter 2 was subjected to classifier analysis, with the aim of identifying classifier genes that are capable of distinguishing between direct immunotoxic and nonimmunotoxic chemicals. To this end, a set of 27 most differentially regulated genes, whose microarray results had been verified by qRT-PCR, were selected as the candidate classifier genes. These genes are known to be involved in a wide range of mechanisms of action leading to direct immunotoxicity. A training set of in total 44 chemicals was assembled, which included the 31 chemicals that have been tested in Chapter 2 and another set of 13 immunotoxicants. The qRT-PCR data of the 27 genes in response to these 44 chemicals were used for a redundancy analysis in order to determine the minimal number of classifier genes that would be required for an accurate binary classification. The results from this analysis showed that 25 would be an optimal size of the classifier gene set as it gave the highest prediction accuracy, without losing any functional information that was originally covered by the 27 genes. In addition, the classification performance of this gene set was validated by an external verification set that contained 20 new chemicals ( 17 immunotoxic and 3 nonimmunotoxic), which resulted in $88 \%$ sensitivity, $67 \%$ specificity, and $85 \%$ overall accuracy.

Furthermore, based on the Gene Ontology (GO) annotation of the 25 classifier genes, it is also possible to categorize direct immunotoxicants into distinct functional subclasses, including regulation of cell cycle and apoptosis, metabolism and transport of cholesterol, cellular signal transduction, cellular stress responses, immune regulation, protein metabolism, retinoic acid metabolism, and regulation of transcription, which would provide initial information in the hazard characterization step.

\section{Second round validation of the biomarkers}

From the results obtained in Chapter 6, another two important questions were raised. The first one was regarding the prediction performance of this classifier set when challenged by new classes of immunotoxicants. Since it was not possible to cover all classes of immunotoxicants in the training set, it would be relevant to further investigate the performance of this classifier set towards chemical classes that had not been tested yet. In addition, only a limited number of nonimmunotoxic control chemicals were tested in Chapter $\mathbf{6}$, which resulted in a less satisfactory specificity (67\%). Therefore, the second question was how this classifier set would perform in response to more nonimmunotoxic control chemicals. In order to answer these questions, another set of 18 chemicals was selected in Chapter 7, which included nine immunotoxicants, five nonimmunotoxic control chemicals, and four "questionable" chemicals for which human immunotoxicity has not been fully established. The nine immunotoxicants included new chemical classes that had not been tested before, such as the polybrominated flame retardant TBBPA and the biological anti-CD3. Based on the nine 212 
immunotoxicants and five nonimmunotoxic control chemicals, the classifier set gave an even better performance as compared to the first round of validation in Chapter $\mathbf{6}$, being $100 \%$ sensitivity, $80 \%$ specificity, and $93 \%$ overall accuracy.

However, one of the four "questionable" chemicals sunset yellow, which has been shown to specifically affect the proportion of monocytes in mice (Hashem et al., 2010), was classified as nonimmunotoxic. This result highlighted one of the possible limitations of the current assay that it might not be able to detect chemicals that target immune cells other than $\mathrm{T}$ cells. Therefore, this Jurkat cell-based screening assay should not be used as a separate assay, but as part of an integrated testing strategy to screen chemicals for potential immunotoxicity and prioritize chemicals for further testing.

\section{Relevance of the in vitro data to human risk}

In order to estimate the potential relevance of these in vitro data to risks in humans in vivo, it is of great importance to compare the concentrations applied in vitro with concentrations in humans in vivo. This comparison was done in Chapter 2 and indicated that for some crucial test chemicals, being the immunomodulators As2O3, DON, TBTC, TBTO, FTY720, mycophenolic acid, cyclosporine A, rapamycin, and the non-immunotoxic control AgNO3, furosemide, and azathioprine, the concentrations used for in vitro exposure were comparable $(0.33-3 x)$ or even lower $(<0.33 x)$ then the concentrations found in systemic circulation or $\mathrm{ADI} / \mathrm{TDI} / \mathrm{RfD}$ in humans in vivo (Table 1). 
TABLE 1. Comparison of the in vitro concentrations used in our study with the physiological concentrations in human in vivo.

\begin{tabular}{|c|c|c|c|c|}
\hline Chemical & $\begin{array}{l}\text { Exposure } \\
\text { conc. in } \\
\text { Jurkat } \\
(\mu \mathrm{M})\end{array}$ & $\begin{array}{l}\text { Physiological conc. in } \\
\text { human }(\mu \mathrm{M})\end{array}$ & References & Comparison \\
\hline $\mathrm{As}_{2} \mathrm{O}_{3}$ & 3 & $\begin{array}{c}\text { in urine, }<0.14 \text { (Europe \& US), } \\
0.7 \text { (Japan), }>14 \text { (West } \\
\text { Bengal, India and Bangladesh) }\end{array}$ & $\begin{array}{l}\text { (Kristiansen et al., 1997); (Kavanagh et al., } \\
\text { 1998) (Europe). (Bates et al., 1995); (Lewis } \\
\text { et al., 1999) (US). (Yamauchi et al., 1992) } \\
\text { (Japan). (Das et al., 1994) (West Bengal, } \\
\text { India and Bangladesh) }\end{array}$ & \\
\hline DON & 0.25 & $\begin{array}{c}\text { estimated TDI, } 1 \text { to } 1000 \mu \mathrm{g} / \mathrm{kg} \\
\text { body weight/day }(0.003375 \text { to } \\
3.37)\end{array}$ & (Katika et al., 2012) & \\
\hline $\begin{array}{l}\text { TBTC } \\
\text { TBTO }\end{array}$ & $\begin{array}{l}0.1 \\
0.1\end{array}$ & $\begin{array}{c}\text { total butyltin levels, in plasma, } \\
0.2 \text { (US) } 0.16 \text { (Japan) }\end{array}$ & $\begin{array}{c}\text { (Whalen et al., 1999) (US); (Takahashi et } \\
\text { al., 1999) (Japan) }\end{array}$ & \\
\hline FTY720 & 4 & $\begin{array}{c}\text { in plasma, } 0.51 \text { to } 9.2 \text { (after } \\
\text { signle oral dose of } 0.25 \text { to } 3.5 \\
\mathrm{mg} \text { ) }\end{array}$ & (Budde et al., 2002) & $\begin{array}{l}\text { in vitro } \\
\text { comparable to in } \\
\text { vivo: } 0.33-3.0 \mathrm{x}\end{array}$ \\
\hline MPA & 10 & $\begin{array}{l}\text { in plasma, } 8.43 \text { to } 83.3 \text { (after } \\
\text { single oral dose of } 100 \text { to } 1000 \\
\text { mg mycophenolate mofetil) }\end{array}$ & (Vethe et al., 2008) & \\
\hline $\mathrm{AgNO}_{3}$ & 1.35 & $\begin{array}{l}\text { in plasma, } 1.67 \text { (concentration } \\
\text { of silver, in patients after } \\
\text { application of Silver } \\
\text { sulfadiazine cream) }\end{array}$ & (Wan et al., 1991) & \\
\hline AZA & 0.1 & $\begin{array}{c}\text { in plasma, } 0.21 \text { to } 0.43 \\
\text { (concentration of } 6-\mathrm{MP}, \text { after } \\
\text { single oral dose of } 100 \mathrm{mg} \\
\text { AZA) }\end{array}$ & (Minegishi et al., 2006) & \\
\hline $\mathrm{BaP}$ & $\begin{array}{c}5(1261.55 \\
\mu \mathrm{g} / \mathrm{l})\end{array}$ & $\begin{array}{c}\text { total PAH levels, in plasma, } \\
23.96 \text { to } 125.55 \mu \mathrm{g} / \mathrm{l} \text { (Indian } \\
\text { children) }\end{array}$ & (Singh et al., 2008) & \\
\hline $\mathrm{CoCl}_{2}$ & 80 & $\begin{array}{c}\text { in plasma, } 0.044 \text { to } 0.095 \\
\text { (patients with metal-on-metal } \\
\text { hip replacement) }\end{array}$ & (Adami et al., 2003) & \\
\hline DZN & 200 & $\begin{array}{l}\text { in plasma, } 0 \text { to } 0.0016 \text { (women } \\
\text { and newborns living in } \\
\text { agricultural community in US) }\end{array}$ & (Huen et al., 2012) & $\begin{array}{l}\text { in vitro higher } \\
\text { than in vivo: }\end{array}$ \\
\hline LIN & 130 & $\begin{array}{c}0.3 \mu \mathrm{g} / \mathrm{kg} \text { body weight (RfD, } \\
\text { US EPA) (0.001032) }\end{array}$ & (EPA) & $>3.0 x$ \\
\hline $\mathrm{MeHg}$ & 1 & $\begin{array}{c}\text { in plasma, } 0.0496 \text { to } 0.057 \\
\text { (Inuit population of Nunavik, } \\
\text { Quebec, Canada) }\end{array}$ & (Ayotte et al., 2011) & \\
\hline MEHP & 300 & $\begin{array}{l}\text { in plasma, } 0 \text { to } 5.5 \text { (in } \\
\text { maternal and cord plasmas, } \\
\text { Italy) }\end{array}$ & (Latini et al., 2003) & \\
\hline
\end{tabular}




\begin{tabular}{|c|c|c|c|c|}
\hline OTA & 10 & $\begin{array}{l}\text { in plasma, } 0.00025 \text { to } 0.014 \\
\text { (Spain and Germany) }\end{array}$ & $\begin{array}{c}\text { (Medina et al., 2010) (Spain); (Studer-Rohr } \\
\text { I, 2000) (Germany) }\end{array}$ & \\
\hline PROP & 100 & $\begin{array}{l}5 \mu \mathrm{g} / \mathrm{kg} \text { body weight (RfD, US } \\
\text { EPA) }(0.022927)\end{array}$ & $(\mathrm{EPA})$ & \\
\hline $\mathrm{CP}$ & 3000 & $\begin{array}{c}\text { in plasma, } 4.7 \text { (after single IV } \\
\text { dose of } 20 \mathrm{mg} / \mathrm{kg} \text { ) }\end{array}$ & (Wagner et al., 1981) & \\
\hline PRD & 500 & $\begin{array}{c}\text { in plasma, } 0.42 \text { (after single IV } \\
\text { dose of } 7.5 \mathrm{mg})\end{array}$ & (Russell et al., 2010) & \\
\hline AMP & 850 & $\begin{array}{l}\text { in plasma, } 4.9 \text { (after single } \\
\text { injection of } 250 \mathrm{mg} \text { into } \\
\text { trachea) }\end{array}$ & (Maddocks, 1975) & \\
\hline FLX & 30 & $\begin{array}{l}\text { in plasma, } 0.02 \text { to } 0.36 \text { (after } 6 \\
\text { weeks of treatment, } 20 \mathrm{mg} / \\
\text { day); in plasma, } 0.05 \text { (after } \\
\text { single oral dose of } 20 \mathrm{mg} \text { ) }\end{array}$ & (Bonne et al., 1999); (Keller et al., 2005) & \\
\hline MAN & 2000 & $\begin{array}{l}\text { estimated ADI, } 0 \text { to } 50 \mathrm{mg} / \mathrm{kg} \\
\text { body weight/day ( } 0 \text { to } 274.47 \text { ) }\end{array}$ & (WHO) & \\
\hline CsA & 8 & $\begin{array}{c}\text { in plasma, } 1298.01(\mathrm{after} \\
\text { single oral dose of } 5 \mathrm{mg} / \mathrm{kg})\end{array}$ & (Klawitter et al., 2010) & \\
\hline RAPA & 0.02 & $\begin{array}{l}\text { in plasma, } 27.7 \text { (after single } \\
\text { oral dose of } 6 \mathrm{mg} \text { ) }\end{array}$ & (Leelahavanichkul et al., 2005) & $\begin{array}{l}\text { in vitro lower } \\
\text { than in vivo: } \\
\quad<0.33 \mathrm{x}\end{array}$ \\
\hline FURO & 100 & $\begin{array}{l}\text { in plasma, } 1028 \text { to } 22377 \\
\text { (after single IV dose of } 40 \mathrm{mg} \text { ) }\end{array}$ & & \\
\hline NaCitr & 2000 & & & \\
\hline urethane & 20000 & & & \\
\hline
\end{tabular}

Note. The plasma concentrations summarized in the table are the $C_{\max }$ after administration of the compound. 


\section{General discussion}

The advances in molecular biology, bioinformatics, and systems biology have provided a number of new scientific tools, which offer great promise for the new paradigm of mechanism-based toxicity testing (Schmidt, 2009). Toxicogenomics, as one of these new tools, has already proved its value in strengthening risk assessment approaches by adding more information on molecular mechanisms of a limited set of immunotoxic chemicals (Baken et al., 2008; Frawley et al., 2011; Katika et al., 2012; Katika et al., 2011).

By conducting a wide survey study with a large variety of chemicals on the transcriptome and protein phosphorylation profile of human T cells in vitro, we have shown that diverse mechanisms of action may underlie chemical-induced direct immunotoxicity. In this respect, direct immunotoxicty differs from skin sensitization (a type of indirect immunotoxicity), where the Nrf2-Keap1 pathway is known to play a predominant role (Natsch, 2010). Therefore, it is envisaged that an assay based on a set of pathways or genes, rather than one single gene, will allow screening chemicals for direct immunotoxic properties.

Besides its application in mechanistic studies, toxicogenomics has also been demonstrated to be useful in the characterization of structural and functional chemical classes and the development of new biomarkers for toxicity endpoints. However, until recently, only one immunotoxicological study had used toxicogenomics for this purpose. Hochstenbach et al., 2010 identified a list of 48 candidate classifier genes as biomarkers for direct immunotoxicity after exposing human PBMCs to 12 chemicals (Hochstenbach et al., 2010). In this study, Recursive Feature Elimination based on a Support Vector Machine (SVM-RFE) algorithm was applied and resulted in the identification of 48 biomarker genes. Although the biological interpretation of the 48 genes revealed pathways that were associated with the immune system, the risk of not identifying biologically meaningful biomarkers by applying traditional statistical methods cannot be neglected (Chen et al., 2009). Therefore, starting from identification of toxicologically relevant pathways, followed by selection of genes that are representative for each of the pathways, as described in the current thesis, would be a better approach for the identification of biomarkers with functional information. In addition, the biomarkers that are identified in this way can not only classify chemicals by their toxicity, but also categorize chemicals into distinct functional subclasses based on their potential mechanisms of action, which also provides additionally useful information for risk assessment.

Another benefit that comes from applying toxicogenomics in in vitro systems is that it allows direct evaluation of the difference in responses to toxicants across species in a qualitative and quantitative way. Black and co-workers performed transcriptome analysis on TCDD-treated rat and human primary hepatocytes (Black et al., 2012). Significant changes in expression were observed between the two species at both gene and pathway levels. By applying a benchmark dose analysis on the dose-dependent transcriptional induction of aryl hydrocarbon receptor regulated genes, they found out an average of 18-fold difference in potency among differentially expressed orthologs in rat and human, with rat being more sensitive than human. These data demonstrated the usefulness of 216 
transcriptional profiling of toxicant-treated primary cells in determining the qualitative and quantitative differences in toxicant responses across species, which will contribute significantly to the reduction or entire removal of the uncertainty factors that are currently used in cross species extrapolation in risk assessment (Rowlands et al., 2014).

The major advantage of using in vitro cell models is that the toxicity of chemicals can be assessed at a much broader range of doses and time points in a cost effective and time saving manner. They can also provide useful information at the level of cellular functions and biochemical pathways that can be affected by chemical exposure. Compared to human primary cells, more reproducible results could be obtained with in vitro cell models because of less genetic variation and a much lower baseline level of environmental pollutants (or immunomodulating medications in terms of immunotoxicological studies). Furthermore, it is much easier to perform toxicity tests in a high throughput format with cell models, especially with a single cell type-based system.

Despite their advantages, several limitations do exist for in vitro cell models. For instance, the extensive interrelationships and crosstalk among multiple cell types in intact functional organs are lacking, especially in single cell type-based models, which makes it impossible to use any single in vitro system to fully mimic the in vivo situation (Astashkina et al., 2012). Furthermore, it is known that many toxicants undergo biotransformation in vivo before exerting their toxic effects. However, for most in vitro cell line models, the metabolic capacities are low. Therefore, it is recommended to perform in vitro incubation of the test chemicals with microsomes, S9 fractions or supersomes before the toxic effect evaluation (Schmidt, 2009).

The predictive assay described in the current thesis could contribute to the evaluation of lymphotoxicity, which is the proposed second tier in the in vitro evaluation of direct immunotoxicity according to Lankveld and co-workers (Lankveld et al., 2010). As also stated in the introduction section of this thesis, the most reliable in vitro functional tests for this purpose nowadays are the whole blood cytokine release assay and the lymphocyte proliferation assay (Lankveld et al., 2010). Compared to these two tests, the major advantages of the present assay are that 1) it has been validated by a large number of chemicals that cover a variety of chemical structures and classes, which ensures a better prediction performance when challenged by new chemicals in the future, and 2 ) it provides functional information regarding the mechanisms of action of chemicals at molecular level. However, the present assay may not allow the assessment of the immunotoxic potency of the test chemicals because of the complexity of the endpoints it measures (in terms of genes and pathways), whereas in the other two assays, the relative potency could be established by the calculation of $\mathrm{IC}_{50}$ (i.e. the $50 \%$ inhibitory concentration) or $\mathrm{SC}_{4}$ (i.e. the fourfold stimulating concentration). Therefore, this limitation again highlights the fact that this RNA signature-based assay should not be viewed as a separate assay, but as part of the in vitro test battery to screen chemicals for their potential immunotoxic properties and prioritize chemicals for further testing, which eventually contributes to the refinement, reduction and ultimately replacement of animal use in toxicological risk assessment. 


\section{Future perspectives}

The Jurkat screening assay has great promise to be incorporated into animal-free testing of human immunotoxicity. However, for a more proper validation of the assay, more nonimmunotoxic chemicals should be tested in order to achieve the balance between immunotoxic and nonimmunotoxic chemicals, although it is difficult to find true negative controls. Furthermore, it would be interesting to challenge this assay with false positive and false negative chemicals generated from in vivo studies, in order to compare the prediction performance of this assay with traditional animal-based approaches. And although in the second round of validation, the Jurkat assay proved its applicability when challenging with new classes of chemicals that were not included in the training set, it does not rule out the possibility that additional classifier genes might need to be added to the current classifier gene set in order to enhance the sensitivity against novel classes of chemicals. The next step for the development of an appropriate screening test would be submitting the assay to EURL-ECVAM for further validation. This will include the assessment of its predictive capacity and reproducibility within and between different test facilities. The second aspect may be relatively easy addressed since 1) Jurkat is a widely used and commercially available cell line that can be cultured in standard medium; 2) the qRT-PCR assays and the primers we used are commercially available; 3) the qRT-PCR experiments were already outsourced and were performed with certified SOPs.

In the current thesis, an antibody array based approach was applied to study the mechanisms involved in chemical-induced immunotoxicity. This approach has been proven to be sensitive and fast in the identification of changes induced by chemical exposure at phosphoproteome level. However, due to the limited number of phosphoproteins that are tested on the array, it could be that some relevant targets were missed. Based on the results of the antibody array, it can be concluded that some of the immunotoxicants could alter protein phosphorylation in Jurkat cells at earlier time points ( $3 \mathrm{~h}$ or even $0.5 \mathrm{~h}$ ). Therefore, it would be interesting to use phosphoproteomics to screen a set of immunomodulating chemicals, in order to examine whether it is possible to identify biomarkers that can be used for measurements at shorter exposure time points.

\section{Overall conclusion}

On the basis of the results presented in the current thesis, it can be concluded that the application of toxicogenomics to in vitro cell models provides valuable information regarding the molecular mechanisms of action of immunotoxicants. Furthermore, the results demonstrated the usefulness of toxicogenomics for the development of a predictive screening assay for direct immunotoxicity. This assay would be useful in the identification and initial characterization of immunotoxic hazards of existing and novel chemicals and prioritizing them for further (regulatory) testing. However, given the multi-organ/ cell type nature of the immune system and the diversity of molecular mechanisms involved in chemical-induced immunotoxicity, it is envisaged that a battery of in vitro assays, instead of one single test, will be needed to screen chemicals for their immunotoxic properties. 


\section{References}

Adami, G., Smarrelli, D., Martinelli, B., Acquavita, A., and Reisenhofer, E. (2003). Cobalt blood levels after total hip replacement (THR): a new follow-up study in Trieste (Italy). Ann. Chim 93(1-2), 1-10.

Astashkina, A., Mann, B., and Grainger, D. W. (2012). A critical evaluation of in vitro cell culture models for high-throughput drug screening and toxicity. Pharmacology \& Therapeutics 134(1), 82106.

Ayotte, P., Carrier, A., Ouellet, N., Boiteau, V., Abdous, B., Sidi, E., Château-Degat, M., and Dewailly, É. (2011). Relation between methylmercury exposure and plasma paraoxonase activity in inuit adults from Nunavik. Environ Health Perspect 119(8), 1077-83.

Baken, K. A., Pennings, J. L. A., Jonker, M. J., Schaap, M. M., de Vries, A., van Steeg, H., Breit, T. M., and van Loveren, H. (2008). Overlapping gene expression profiles of model compounds provide opportunities for immunotoxicity screening. Toxicology and Applied Pharmacology 226(1), 46-59.

Bates, M. N., Smith, A. H., and Cantor, K. P. (1995). Case-control study of bladder cancer and arsenic in drinking water. American Journal of Epidemiology 141(6), 523-530.

Black, M. B., Budinsky, R. A., Dombkowski, A., Cukovic, D., LeCluyse, E. L., Ferguson, S. S., Thomas, R. S., and Rowlands, J. C. (2012). Cross-species comparisons of transcriptomic alterations in human and rat primary hepatocytes exposed to 2,3,7,8-tetrachlorodibenzo-p-dioxin. Toxicological Sciences 127(1), 199-215.

Bonne, O., Krausz, Y., Aharon, Y., Gelfin, Y., Chisin, R., and Lerer, B. (1999). Clinical doses of fluoxetine and cerebral blood flow in healthy volunteers 143(1), 24-28.

Budde, K., Schmouder, R. L., Brunkhorst, R., Nashan, B., Lücker, P. W., Mayer, T., Choudhury, S., Skerjanec, A., Kraus, G., and Neumayer, H. H. (2002). First human trial of FTY720, a novel immunomodulator, in stable renal transplant patients. Journal of the American Society of Nephrology 13(4), 1073-1083.

Chen, L., Xuan, J., Wang, C., Wang, Y., Shih, I., Wang, T., Zhang, Z., Clarke, R., and Hoffman, E. (2009). Biomarker identification by knowledge-driven multilevel ICA and motif analysis. Int J Data Min Bioinform 3(4), 365-81.

Chung, J., Kuo, C. J., Crabtree, G. R., and Blenis, J. (1992). Rapamycin-FKBP specifically blocks growth-dependent activation of and signaling by the $70 \mathrm{kd} \mathrm{S6}$ protein kinases. Cell 69(7), 1227-1236.

Das, D., Chatterjee, A., Samanta, G., Mandal, B., Chowdhury, T., Samanta, G., Chowdhury, P., Chanda, C., Basu, G., and Lodh, D. (1994). Arsenic contamination in groundwater in six districts of West Bengal, India: the biggest arsenic calamity in the world. Analyst 119(12), 168-170.

EPA, U. S. Lindane. Available at: http://www.epa.gov/ttnatw01/hlthef/lindane.html.

EPA, U. S. Propanil. Available at: http://www.epa.gov/iris/subst/0186.htm.

Frawley, R., White, K., Brown, R., Musgrove, D., Walker, N., and Germolec, D. (2011). Gene expression alterations in immune system pathways in the thymus after exposure to immunosuppressive chemicals. Environ Health Perspect 119(3), 371-376. 
Hashem, M. M., Atta, A. H., Arbid, M. S., Nada, S. A., and Asaad, G. F. (2010). Immunological studies on Amaranth, Sunset Yellow and Curcumin as food colouring agents in albino rats. Food and Chemical Toxicology 48(6), 1581-1586.

Hochstenbach, K., van Leeuwen, D., Gmuender, H., Gottschalk, R., Stølevik, S. B., Nygaard, U. C., Lovik, M., Granum, B., Namork, E., Meltzer, H. M., Kleinjans, J., van Delft, J., and van Loveren, H. (2012). Toxicogenomic profiles in relation to maternal immunotoxic exposure and immune functionality in newborns. Toxicological Sciences 129(2), 315-24.

Hochstenbach, K., van Leeuwen, D. M., Gmuender, H., Stølevik, S. B., Nygaard, U. C., Løvik, M., Granum, B., Namork, E., van Delft, J. H. M., and van Loveren, H. (2010). Transcriptomic profile indicative of immunotoxic exposure: in vitro studies in peripheral blood mononuclear cells. Toxicological Sciences 118(1), 19-30.

Huen, K., Bradman, A., Harley, K., Yousefi, P., Boyd Barr, D., Eskenazi, B., and Holland, N. (2012). Organophosphate pesticide levels in blood and urine of women and newborns living in an agricultural community. Environmental Research 117(0), 8-16.

Katika, M. R., Hendriksen, P. J. M., Shao, J., van Loveren, H., and Peijnenburg, A. (2012). Transcriptome analysis of the human $\mathrm{T}$ lymphocyte cell line Jurkat and human peripheral blood mononuclear cells exposed to deoxynivalenol (DON): New mechanistic insights. Toxicology and Applied Pharmacology 264(1), 51-64.

Katika, M. R., Hendriksen, P. J. M., van Loveren, H., and Peijnenburg, A. (2011). Exposure of Jurkat cells to bis (tri-n-butyltin) oxide (TBTO) induces transcriptomics changes indicative for ER- and oxidative stress, T cell activation and apoptosis. Toxicology and Applied Pharmacology 254(3), 311322.

Kavanagh, P., Farago, M., Thornton, I., Goessler, W., Kuehnelt, D., Schlagenhaufen, C., and Irgolic, K. (1998). Urinary arsenic species in Devon and Cornwall residents, UK. A pilot study. Analyst 123(1), 27-9.

Keller, T., Cambon, N., Genevray, M., Crivelli, F., Crivelli, M., Dal, B., Mazzucchelli, P., Ismaili, S., and Marzo, A. (2005). Bioequivalence study of fluoxetine hydrochloride in healthy volunteers. Arzneimittelforschung 55(9), 491-7.

Klawitter, J., Haschke, M., Kahle, C., Dingmann, C., Klawitter, J., Leibfritz, D., and Christians, U. (2010). Toxicodynamic effects of ciclosporin are reflected by metabolite profiles in the urine of healthy individuals after a single dose. British Journal of Clinical Pharmacology 70(2), 241-251.

Kristiansen, J., Christensen, J. M., Iversen, B. S., and Sabbioni, E. (1997). Toxic trace element reference levels in blood and urine: influence of gender and lifestyle factors. Science of The Total Environment 204(2), 147-160.

Lankveld, D. P. K., Loveren, H., Baken, K. A., Vandebriel, R. J., and Dietert, R. R. (2010). In Vitro Testing for direct immunotoxicity: state of the art immunotoxicity testing. In Immunotoxicity Testing (J. M. Walker, Ed.), Vol. 598, pp. 401-423. Humana Press.

Latini, G., De Felice, C., Presta, G., Del Vecchio, A., Paris, I., Ruggieri, F., and Mazzeo, P. (2003). Exposure to di(2-ethylhexyl)phthalate in humans during pregnancy. Neonatology 83(1), 22-24. 
Leelahavanichkul, A., Areepium, N., Vadcharavivad, S., Praditpornsilpa, K., Avihingsanon, Y., Karnjanabuchmd, T., Eiam-Ong, S., and Tungsanga, K. (2005). Pharmacokinetics of sirolimus in Thai healthy volunteers. J Med Assoc Thai 88(Suppl 4), S157-62.

Lewis, D., Southwick, J., Ouellet-Hellstrom, R., Rench, J., and Calderon, R. (1999). Drinking water arsenic in Utah: A cohort mortality study. Environ Health Perspect 107(5), 359-65.

Maddocks, J. (1975). Absorption of ampicillin from the human lung. Thorax 30(1), 68-71.

Medina, Á., Mateo, E. M., Roig, R. J., Blanquer, A., and Jiménez, M. (2010). Ochratoxin A levels in the plasma of healthy blood donors from Valencia and estimation of exposure degree: comparison with previous national Spanish data. Food Additives \& Contaminants: Part A 27(9), 1273-1284.

Minegishi, Y., Saito, M., Morio, T., Watanabe, K., Agematsu, K., Tsuchiya, S., Takada, H., Hara, T., Kawamura, N., Ariga, T., Kaneko, H., Kondo, N., Tsuge, I., Yachie, A., Sakiyama, Y., Iwata, T., Bessho, F., Ohishi, T., Joh, K., Imai, K., Kogawa, K., Shinohara, M., Fujieda, M., Wakiguchi, H., Pasic, S., Abinun, M., Ochs, H. D., Renner, E. D., Jansson, A., Belohradsky, B. H., Metin, A., Shimizu, N., Mizutani, S., Miyawaki, T., Nonoyama, S., and Karasuyama, H. (2006). Human tyrosine kinase 2 deficiency reveals its requisite roles in multiple cytokine signals involved in innate and acquired immunity. Immunity 25(5), 745-755.

Natsch, A. (2010). The Nrf2-Keap1-ARE toxicity pathway as a cellular sensor for skin sensitizersfunctional relevance and a hypothesis on innate reactions to skin sensitizers. Toxicological Sciences 113(2), 284-292.

Rowlands, J. C., Sander, M., Bus, J. S., and Committee, F. O. (2014). FutureTox: building the road for 21st century toxicology and risk assessment practices. Toxicological Sciences 137(2), 269-277.

Russell, G. M., Henley, D. E., Leendertz, J., Douthwaite, J. A., Wood, S. A., Stevens, A., Woltersdorf, W. W., Peeters, B. W. M. M., Ruigt, G. S. F., White, A., Veldhuis, J. D., and Lightman, S. L. (2010). Rapid glucocorticoid receptor-mediated inhibition of hypothalamic-pituitary-adrenal ultradian activity in healthy males. The Journal of Neuroscience 30(17), 6106-6115.

Schmidt, C. W. (2009). TOX21: new dimensions of toxicity testing. Environ Health Perspect 117(8), A348-A353.

Singh, V. K., Patel, D. K., Jyoti, Ram, S., Mathur, N., and Siddiqui, M. K. J. (2008). Blood levels of polycyclic aromatic hydrocarbons in children and their association with oxidative stress indices: An Indian perspective. Clinical Biochemistry 41(3), 152-161.

Smith, D. E., Lin, E. T., and Benet, L. Z. (1980). Absorption and disposition of furosemide in healthy volunteers, measured with a metabolite-specific assay. Drug Metabolism and Disposition 8(5), 337342.

Studer-Rohr, I., Schlatter, J., and Dietrich, D. R. (2000). Kinetic parameters and intraindividual fluctuations of ochratoxin A plasma levels in humans. Arch Toxicol 74(9), 499-510.

Takahashi, S., Mukai, H., Tanabe, S., Sakayama, K., Miyazaki, T., and Masuno, H. (1999). Butyltin residues in livers of humans and wild terrestrial mammals and in plastic products. Environmental Pollution 106(2), 213-218.

Tugwood, J. D., Hollins, L. E., and Cockerill, M. J. (2003). Genomics and the search for novel biomarkers in toxicology. Biomarkers 8(2), 79-92. 
Vandebriel, R. J., Pennings, J. L. A., Baken, K. A., Pronk, T. E., Boorsma, A., Gottschalk, R., and Van Loveren, H. (2010). Keratinocyte gene expression profiles discriminate sensitizing and irritating compounds. Toxicological Sciences 117(1), 81-89.

Vethe, N., Bremer, S., Rootwelt, H., and Bergan, S. (2008). Pharmacodynamics of mycophenolic acid in CD4+ cells: a single-dose study of IMPDH and purine nucleotide responses in healthy individuals. Ther Drug Monit 30(6), 645-55.

Wagner, T., Heydrich, D., Jork, T., Voelcker, G., and Hohorst, H. (1981). Comparative study on human pharmacokinetics of activated ifosfamide and cyclophosphamide by a modified fluorometric test. J Cancer Res Clin Oncol 100(1), 95-104.

Wan, A. T., Conyers, R. A., Coombs, C. J., and Masterton, J. P. (1991). Determination of silver in blood, urine, and tissues of volunteers and burn patients. Clinical Chemistry 37(10), 1683-7.

Waters, M. D., and Fostel, J. M. (2004). Toxicogenomics and systems toxicology: aims and prospects. Nat Rev Genet 5(12), 936-948.

Whalen, M. M., Loganathan, B. G., and Kannan, K. (1999). Immunotoxicity of environmentally relevant concentrations of butyltins on human natural killer cells in vitro. Environmental Research 81(2), 108-116.

WHO Food Additives Series 21 Available http://www.inchem.org/documents/jecfa/jecmono/v21je10.htm.

Yamauchi, H., Takahashi, K., Mashiko, M., Saitoh, J., and Yamamura, Y. (1992). Intake of different chemical species of dietary arsenic by the japanese, and their blood and urinary arsenic levels. Applied Organometallic Chemistry 6(4), 383-388. 


\section{CHAPTER 9}

Samenvatting (Summary in Dutch) 
Het doel van het werk beschreven in dit proefschrift was tweeledig. Ten eerste, het identificeren van werkingsmechanismen die betrokken zijn bij directe immunotoxiciteit; ten tweede, het identificeren van functionele biomerkers welke vervolgens gebruikt kunnen worden voor het ontwikkelen van een in vitro assay voor het voorspellen van directe immunotoxiciteit. Hoofdstuk 1 geeft een overzicht van het immuunsysteem, definities die gebruikt worden in het onderzoeksveld van de immunotoxicologie, huidige in vitro en in vivo testen voor het vaststellen van immunotoxiciteit en de huidige stand van zaken met betrekking tot het toepassen van -omics technieken binnen de immunotoxicologie (immunotoxicogenomics). In het licht van de bovenvermelde doelstellingen, zijn de overige hoofdstukken van het proefschrift (Hoofdstuk 2-7) verdeeld over twee secties.

Sectie 1 is gericht op het in kaart brengen van cellulaire processen die bij verstoring door chemicaliën kunnen resulteren in direct immunotoxiciteit. In Hoofdstuk 2 is de humane T lymfocyt cellijn Jurkat blootgesteld aan een groot aantal stoffen waaronder immunotoxische contaminanten, immunosuppressieve medicijnen en niet-immunotoxische controle chemicaliën en zijn de effecten op genoom-wijde mRNA expressie (oftewel transcriptoom) bestudeerd met behulp van DNA microarrays. Deze studie heeft geleid tot de identificatie van verscheidene werkingsmechanismen waarvan een aantal gedeeld werden door meerdere chemicaliën. In Hoofdstuk $\mathbf{3}$ is vervolgens van een subset van deze stoffen nagegaan of deze effecten hebben op de fosforylering van eiwitten waarvan bekend is dat ze een belangrijke rol spelen in cellulaire signaleringsroutes. Daartoe zijn eiwitextracten van blootgestelde Jurkat cellen geanalyseerd met behulp van antilichaam arrays. Daar een van de gemeenschappelijk gemoduleerde signaleringsroutes in de richting wees van celmigratie, werd het effect van een aantal immunotoxische stoffen op dit proces nader bestudeerd met een functionele chemotaxis assay (Hoofdstuk 4). In Hoofdstuk 5 is, aan de hand van TBTO als model immunotoxische stof, een microarray studie uitgevoerd met Perifere Bloed Mononucleaire Cellen (PBMCs) met als doel om na te gaan of de in de Jurkat cellijn veranderde processen ook in humane primaire immuuncellen zouden optreden.

In Sectie 2 worden de identificatie en validatie van biomerker genen voor directe immunotoxiciteit beschreven. In Hoofdstuk 6 zijn de microarray data die beschreven zijn in Hoofdstuk 2 verder geanalyseerd om genen te identificeren die gebruikt kunnen worden voor het classificeren van stoffen als immunotoxisch of niet-immunotoxisch. De geidentificeerde genen zijn verder op waarde beoordeeld in validatie experimenten met nieuwe sets van immunotoxische en niet-immunotoxicshe chemicaliën (Hoofdstukken 6 en 7).

\section{Potentiele mechanismen die ten grondslag liggen aan directe immunotoxiciteit}

Identificatie van werkingsmechanismen door middel van transcriptoom analyse

Onlangs zijn een aantal in vitro en in vivo studies gepubliceerd waarin via een toxicogenomics benadering meer inzicht verkregen is in de werkingsmechanismen van immunotoxische stoffen. Het ging in deze studies echter om een beperkt aantal stoffen waaronder het biocide TBTO en het 224 
mycotoxine DON. In Hoofdstuk 2 zijn de effecten van een groot aantal verschillende chemicaliën op het transcriptoom van de humane Jurkat $T$ cellijn bestudeerd. Deze chemicaliën (in totaal 31) betroffen milieu contaminanten, zware metalen, pesticiden, biociden, mycotoxinen, immunosppressieve geneesmiddelen en niet-immunotoxische controle chemicaliën. $\mathrm{Na}$ bioinformatische analyse van de transcriptoom data kon vastgesteld worden dat meerdere chemicaliën $(>3)$ invloed hadden op dezelfde biologische processen, zoals ER stress, oxydatieve stress, anti-apotose, controle van de cell cyclus, metabolisme van cholesterol en lipiden en regulatie van transcriptie en translatie. Verder werden processen, zoals Notch signalering, NF-KB signalering en RXR/RAR signalering specifiek beïnvloed door één of twee immunotoxische stoffen. Een aantal van deze processen werd ook reeds geidentificeerd in de eerdere toxicogenomics studies. Dat laatste geldt niet voor de modulatie van lipiden/cholesterol metabolisme, RXR/RAR signalering en Notch signalering. Deze zijn nieuwe voor directe immunotoxiciteit potentieel relevante processen.

Identificatie van werkingsmechanismen door middel van profilering van eiwit fosforylering

Hoofdstuk 3 beschrijft onderzoek naar de effecten van een subset van de in Hoofdstuk 2 onderzochte chemicaliën op de fosforylering van eiwitten, in het bijzonder kinases, in Jurkat cellen met behulp van receptor tyrosine kinase (RTK) antilichaam arrays. De subset van chemicaliën bestond uit vijf immunotoxische stoffen (lindaan, ochratoxine A, TBTC, TBTO en DON), twee immunosuppressieve medicijnen (rapamycine en mycofenolzuur) en twee niet-immunotoxische controle chemicaliën (urethaan en mannitol). Een aantal van de immuotoxische/immunosuprresieve stoffen bleken de fosforylering van dezelfde kinases te beinvloeden. Met name het ribosomale eiwit S6 werd door meerdere chemicaliën, inclusief TBTO, gefosforyleerd. De resultaten verkregen met TBTO konden vervolgens bevestigd worden met flow cytometrie en Western blotting. Van RPS6 is bekend dat het een belangrijk downstream effector is van de mTOR-p70S6K-RPS6 route. Vervolgonderzoek richtte zich dan ook het verkrijgen van meer inzicht in het effect van TBTO op deze route. Daarbij werd ook rapamycine meegenomen omdat deze stof bekend staat als een selectieve remmer van de kinase mTOR. Behandeling van Jurkat cellen met TBTO resulteerde in defosforylering van p70S6K maar had geen effect op de fosforylering van mTOR; rapamycin behandeling leidde echter tot defosforylering van mTOR en p70S6K. Deze bevinding duidde er op dat zowel TBTO als rapamycine de p70S6K route remmen, maar via verschillende moleculaire mechanismen. Dit resultaat suggereert dat, hoewel verschillende van de onderzochte stoffen een effect hebben op de p70S6K route, de primaire aangrijpingspunten van deze stoffen op verschillende niveaus van de route kunnen liggen. 
Vergelijking van de trancriptoom en eiwit fosforylering data

In Hoofdstuk $\mathbf{3}$ is een vergelijking gemaakt van de microarray data en de eiwit fosforylering data die verkregen zijn na behandeling van Jurkat cellen met TBTO. Beide typen data doen vermoeden dat TBTO ribosoom biogenese en celmigratie processen kan beïnvloeden.

Functionele assay: in vitro trans-well chemotaxis assay

In Hoofdstuk 3 zijn experimenten gedaan om het mogelijk effect van TBTO op celmigratie verder te onderzoeken. Daartoe is een in vitro trans-well chemotaxis assay opgezet waarbij gebruik germaakt werd van Jurkat cellen en het chemokine CXCL12. De resultaten lieten zien dat TBTO de CXCL12 gemedieerde migratie van Jurkat cellen inhibiteerde, wat een bevestiging is van de hypothese geformuleerd op basis van de trancriptoom en eiwit fosforylering data.

In Hoofdstuk $\mathbf{4}$ is de in vitro trans-well chemotaxis assay breder ingezet om andere immunotoxische stoffen te testen op chemotaxis-modulerende eigenschappen. Daartoe is eerst een uitvoerige analyse gedaan van de in Hoofdstuk 2 gegenereerde transcriptoom data met het accent op mRNA expressie van genen waarvan bekend is dat ze betrokken zijn bij celmigratie. Deze analyse resulteerde in de identificatie van zeven chemicaliën waarvan vijf, door te testen in de the trans-well chemotaxis assay, geoormerkt konden worden als chemotaxis remmer. Een andere set van zes chemicaliën, waarvoor op basis van de transcriptoom data geen aanwiizing gevonden kon worden voor een effect op celmigratie, werden eveneens getest met de assay. Een van deze zes stoffen, mycofenolzuur, bleek echter de chemotaxis van Jurkat cellen te remmen. Deze bevinding gaf aan dat CXCL12 gemedieerde chemotaxis niet alleen op transcriptoom niveau gereguleerd wordt. Niettemin lieten de resultaten zien dat sommige immunotoxische stoffen hun effecten op het immuun systeem kunnen uitoefenen door remming van CXCL12 gemedieerde chemotaxis.

\section{Effecten van TBTO op perifere bloed mononucleaire cellen}

Daar de bovengenoemde resultaten verkregen werden met een T-cellijn (Jurkat) was het van belang om na te gaan of vergelijkbare effecten zouden optreden in humane primaire cellen. Hoofdstuk 5 beschrijft experimenten waarin vers geisoleerde perifere bloed mononucleaire cellen (PBMCs) werden blootgesteld aan de model immunotoxische stof TBTO gevolgd door transcriptoom analyse. De microarray data lieten zien dat verschillende biologische processen, zoals ER stress, oxydatieve stress en functioneren van mitochondria beïnvloed worden door TBTO in humane PBMCs. Deze processen werden ook aangedaan door TBTO in de Jurkat cellijn (zie Hoofdstuk 2) wat wijst op een goede correlatie tussen de twee in vitro systemen. Bovendien werd gevonden dat in PBMCs, maar niet in Jurkat cellen, de expressie van genen specifiek voor bepaalde lymfocyten subsets ( $T$, B en NK 
cellen), monocyten, dendritische cellen en granulocyten beïnvloed werd door TBTO. Dit is waarschijnlijk terug te voeren op de heterogene samenstelling van de PBMCs.

\section{Functionele biomerkers ten behoeve van de ontwikkeling van een predictieve assay}

Identificatie en eerste ronde validatie van biomerkers

In Hoofdstuk 6 werden de microarray data (gegenereerd in Hoofdstuk 2) geanalyseerd met het doel genen te identificeren die onderscheid zouden kunnen maken tussen immunotoxische en nietimmunotoxische stoffen. In eerste instantie werden 27 genen geselecteerd als kandidaat biomerkers. Deze genen waren representatief voor de verschillende werkingsmechanismen die mogelijk ten grondslag liggen aan directe immunotoxiciteit. Het effect van de 31 in Hoofdstuk 2 onderzochte stoffen op de expressie van deze 27 genen kon bevestigd worden met qRT-PCR. Vervolgens werd een training set van in totaal 44 chemicaliën samengesteld bestaande uit de 31 eerder gebruikte chemicaliën en 13 additionele immunotoxische stoffen. Na behandeling van Jurkat cellen met deze deze stoffen werden de expressieniveaus van de 27 genen bepaald met qRT-PCR. Vervolgens werd op basis van de qRT-PCR data een redundantie analyse gedaan. De uitkomst van deze analyse was dat minimaal 25 genen nodig zijn om tot de meest accurate classificatie immunotoxisch versus nietimmunotoxisch te komen, zonder daarbij enige functionele informatie van de set van 27 genen te verliezen. De prestatie van de set van 25 genen werd verder onderzocht aan de hand van 20 nieuwe chemicaliën (17 immunotoxische en 3 nonimmunotoxische) wat resulteerde in een sensitiviteit van $88 \%$, een specificiteit van $67 \%$ en een accuraatheid van $85 \%$.

\section{Tweede ronde validatie van de biomerkers}

De prestatie karakteristieken van de 25 genen zoals vastgesteld in Hoofdstuk 6 riepen twee belangrijke vragen op. De eerste had betrekking op de prestatie van de genen set wanneer Jurkat cellen behandeld zouden worden met nieuwe klassen van immunotoxische stoffen. De tweede vraag was hoe de prestatie van de genen set zou zijn wanneer meer niet-immunotoxische chemicaliën meegenomen zouden worden. Immers, in de studie beschreven in Hoofdstuk 6 werd slechts een relatief klein aantal niet-immunotoxische controle chemicaliën getest wat mogelijk de reden was voorde matige specificiteit van $67 \%$. Om deze vragen te beantwoorden werd voor de studie beschreven in Hoofdstuk 7 een set van 18 van chemicaliën geselecteerd bestaande uit 9 immunotoxische stoffen, 5 niet-immunotoxische controle chemicaliën en 4 stoffen waarvan de immunotoxicteit voor de mens nog niet helemaal vastgesteld was. Onder de 9 immunotoxische stoffen bevonden zich nieuwe chemische klassen die in de voorgaande hoofdstukken nog niet getest waren, zoals de gebromeerde vlamvertrager TBBPA en het immunosuppressieve medicijn anti-CD3. Op basis van de resultaten verkregen met de 9 immunotoxische en 5 niet-immunotoxische chemicaliën kon geconcludeerd worden dat de set van 25 genen beter presteerde vergeleken met de 
eerste ronde validatie. De prestatie van de genen set was als volgt: $100 \%$ sensitiviteit, $80 \%$ specificiteit en $93 \%$ accuraatheid.

\section{Algehele conclusie}

De in dit proefschrift beschreven resultaten laten zien dat de toepassing van toxicogenomics in combinatie met in vitro celsystemen waardevolle informatie kan verschaffen over de werkingsmechanismen van immunotoxische stoffen en, middels de identificatie van biomerker genen, een aanzet heeft gegeven voor de ontwikkeling van een voorspellende assay voor directe immunotoxiciteit. Deze assay ziet er beloftevol uit maar heeft mogelijk een aantal beperkingen, zoals het foutief classificeren van chemicaliën die specifiek aangrijpen op andere immuuncellen dan Tlymfocyten. Daarom zou de assay ingezet kunnen worden voor het screenen van bestaande en nieuwe chemicaliën op mogelijke immunotoxische gevaren en het prioriteren van deze stoffen voor verder (regulatoir) onderzoek, niet zozeer als een op zichzelf staande test, maar eerder als onderdeel van een batterij van in vitro assays. 
Acknowledgements 

I would like to take this opportunity to express my sincere gratitude and appreciation to my supervisors, colleagues, friends, and family. Although my name is the only one appeared on the cover and front page, the outcomes and quality of this work would not have been the same without the help and support I received from all of you.

Foremost, I would like to thank my promotor Prof. Henk van Loveren for his vision, dedicated supervision and guidance, especially during the last stage of my PhD. I am truly grateful for his stimulating suggestions and encouragements, which brought me confidence and strengthened my faith during the completion of my thesis. And also many thanks for your great help during my job search.

Secondly, I would like to express my deepest gratitude to my co-promotor Dr. Ad Peijnenburg. Your advice, support, and encouragement helped me through all the ups and downs over the past four years. Thank you for your patience and being a good listener whenever I encountered any problems. And thank you for giving me great freedom to pursue independent work.

To my daily supervisor Dr. Oscar Volger who helped and guided me a lot in practical work, thank you very much for your instructions and valuable contribution to this project. I am grateful to have you as my supervisor and I really learned a lot from you.

To Dr. Peter Hendriksen, thank you very much for your guidance in bioinformatics and your thorough and insightful feedback on individual chapters. Your input definitely improved the quality of this thesis.

I would like to give a special gratitude to my office mates: Astrid, Liza, Si, and Ainhoa, and also to Corina, Jonathan, Danique, and Nathanna. Your company has made my days much brighter and warmer in Wageningen. Especially to Astrid, Liza, and Si, thank you so much for your friendship and support that helped me through difficult times.

A big thank you goes to Peter Schmeits. It was really nice that we started more or less at the same time and worked on similar projects, which made it much easier to share our opinions and experiences. Your suggestions and encouragements made me feel I was not isolated in my research.

And thank you Evelien for your help in qRT-PCR and western blot. I would not have been so "skillful" in the lab without your instructions.

To my former students Inge, Jie, Cencia, and Laura, thank you very much for your hard work and contribution to this thesis. It was truly a pleasure to be your supervisor and to work with all of you.

Furthermore, to all my colleagues at RIKILT (Agata, Angeline, Arjen, Eva, Geert, Gerlof, Gerrit, Hans, Henri, Jac, Jenneke, Jeroen, Lonneke, Madhu, Meike, Richard, Ron, and Toine) thank you very much for your help and support. I really enjoyed my time at RIKILT and this experience will always remain in my memory. 
To all my friends in China, the Netherlands, and other parts of the world, you were always the sources of joy, laughter, and support to me. Especially to the friends that I met in the Netherlands, Hequn, Feifei, Jue, Yinhua, Yin, Jing, Book, Pieter, Natasa, Didi, Ou, Yuki, and Ying, etc., thank you so much for your love and friendship, which has made Wageningen a second home to me.

Last but not least, I would like to thank my parents. My doctoral journey would not have been possible without their love and support. To my devoted husband, Xinxin, I am truly appreciated of all that you have done for me and for our family. You took every responsibility and suffered all the bitterness of being both mum and dad when I was away. I owe my every achievement to you. And Alvin, my little sweet heart, mama is so proud of you since our first meet on the day you were born. I hope that you will also be proud of mama when you are able to read this booklet. Thank you for making our life happier and more meaningful.

Completing my PhD degree is definitely one of the most challenging activities in my first 30 years of life. Luckily I had all of you on my side. 
About the Author 



\section{Curriculum Vitae}

Jia Shao was born on November $1^{\text {st }} 1984$ in Beijing, China. After finishing her secondary education in Beijing Foreign Language School in 2003, she left Beijing for Shanghai to study Biotechnology in Tongji University and obtained her Bachelor degree in 2007. She then came to Wageningen University in The Netherlands for her Master study. The study program was Nutrition and Health, and she was specialized in Food Toxicology. Right after her graduation in 2009, she received an offer from Maastricht University and started her $\mathrm{PhD}$ at the department of Toxicogenomics under the supervision of Prof. Henk van Loveren and Dr. Ad Peijnenburg from RIKILT- Food Safety Institute in Wageningen, where the actual research activities took place. The results obtained during these four years are presented in this thesis. In addition, Jia also completed a Postgraduate Education program in Toxicology, which will result in the registration as a toxicologist within the Netherlands Society of Toxicology (NVT). From September 2014 onward, Jia has been working as a Safety Scientist in Unilever Safety and Environmental Assurance Center (SEAC) in UK. 



\section{Publication List}

Journal articles

Shao, J., Katika, M. R., Schmeits, P. C., Hendriksen, P. J., van Loveren, H., Peijnenburg, A. A., and Volger, O. L., 2013. Toxicogenomics-based identification of mechanisms for direct immunotoxicity. Toxicological Science, 135 (2): 328-346.

Shao, J., Berge, L., Hendriksen, P. J., van Loveren, H., Peijnenburg, A. A., and Volger, O. L., 2014. Transcriptome-based functional classifiers for direct immunotoxicity. Archives of Toxicology, 88(3): 673-89.

Shao, J., Stout, I., Hendriksen, P. J., van Loveren, H., Peijnenburg, A. A., and Volger O. L., The mTOR pathway as a target for the immunotoxicant TBTO and other immunomodulating compounds, submitted.

Shao, J., Stout, I., Volger, O. L., Hendriksen, P. J., van Loveren, H., Peijnenburg, A. A., Inhibition of CXCR4-CXCL12 mediated chemotaxis of $\mathrm{T}$ lymphocytes by direct immunotoxicants, in preparation.

Schmeits, P. C., Shao, J., van der Krieken, D. A., Volger, O. L., van Loveren, H., Peijnenburg, A. A., and Hendriksen, P. J., 2014. Successful validation of genomic biomarkers for human immunotoxicity in Jurkat T cells in vitro. Journal of Applied Toxicology, in press.

Katika M. R., Shao J., Hendriksen P. J., van Loveren H., and Peijnenburg A. A., Microarray analysis of human peripheral blood mononuclear cells (PBMCs) treated with tributyltin-oxide (TBTO), submitted.

Katika M. R., Hendriksen P. J., Shao J., van Loveren H., and Peijnenburg A. A., 2012. Transcriptome analysis of the human T lymphocyte cell line Jurkat and human peripheral blood mononuclear cells exposed to deoxynivalenol (DON): New mechanistic insights. Toxicology and Applied pharmacology, 264(1): 51-64.

Brand, W., Shao, J., Hoek-van den Hil, E. F., van Elk, K. N., Spenkelink, B., de Haan, L. H., Rein, M. J., Dionisi, F., Williamson, G., van Bladeren, P. J., and Rietjens, I. M., 2010, Stereoselective conjugation, transport and bioactivity of S- and R- hesperetin enantiomers in vitro. Journal of Agricultural and Food Chemistry, 58: 6119-6125.

Georgantzopoulou, A., Serchi, T., Leclercq, C. C., Renaut, J., Shao, J., Lankoff, A., Kruszewski, M., Lentzen, E., Crysan, P., Audinot, J. N., Contal, S., Ziebel, J., Guignard, C., Hoffmann, L., Murk, A. J., and Gutleb, A. C., Co-culture model for the evaluation of the effects of silver nanoparticles and ions on the gastrointestinal epithelium, submitted. 


\section{Book Chapter}

Hendriksen, P. J., Schmeits, P. C., van Loveren, H., Shao, J., and Peijnenburg, A. A., 2014. Chapter 20: Mode of action of organotins in immune cells. In Molecular Immunotoxicology, $1^{\text {st }}$ Edition (Corsini and van Loveren Eds.), Wiley-VCH Verlag GmbH \& Co. KGaA, Weinheim, Germany. 


\section{Overview of completed training activities}

Conferences and research meetings

$1^{\text {st }}-4^{\text {th }}$ Netherland Toxicogenomics Center (NTC) Annual Meeting, Amsterdam, the Netherlands, 20092013.

$6^{\text {th }}$ Meeting of the Immunotoxicology and Chemical Allergy Specialty Section (ITCASS), NOTOX, den Bosch, the Netherlands, 2009

Annual Meeting of the NVT, Zeist, the Netherlands, 2010

$51^{\text {st }}$ Annual Meeting of the Society of Toxicology (SOT), San Francisco, USA, 2012

$49^{\text {th }}$ Congress of the European Societies of Toxicology (EUROTOX), Interlaken, Switzerland, 2013

Discipline specific courses (All from Postgraduate Education in Toxicology in the Netherlands)

Toxicogenomics, Maastricht, 2010

Reproductive Toxicology, Utrecht, 2011

Laboratory Animal Science, Utrecht, 2011

Organ Toxicology, Nijmegen, 2012

Pathobiology, Utrecht, 2012

General Toxicology, Wageningen, 2013

Ecotoxicology, Utrecht/ Wageningen, 2013

\section{General courses}

Scientific Writing, Wageningen, the Netherlands, 2010

Project and Time Management, Wageningen, the Netherlands, 2013 
This research was finacially supported by the Netherlands Toxicogenomics Center, the Netherlands Genomics Initiative.

Cover design: Proefschriftmaken.nl II Uitgeverij BOXPress

Thesis layout: Jia Shao

Printed by Proefschriftmaken.nl II Uitgeverij BOXPress

Jia Shao, 2015 